\title{
Short-Time Structural Stability of Compressible Vortex Sheets with Surface Tension
}

\author{
Ben Stevens
}

Communicated by P. Constantin

\begin{abstract}
Assume we start with an initial vortex-sheet configuration which consists of two inviscid fluids with density bounded below flowing smoothly past each other, where a strictly positive fixed coefficient of surface tension produces a surface tension force across the common interface, balanced by the pressure jump. We model the fluids by the compressible Euler equations in three space dimensions with a very general equation of state relating the pressure, entropy and density such that the sound speed is positive. We prove that, for a short time, there exists a unique solution of the equations with the same structure.

The mathematical approach consists of introducing a carefully chosen artificial viscosity-type regularisation which allows one to linearise the system so as to obtain a collection of transport equations for the entropy, pressure and curl together with a parabolic-type equation for the velocity which becomes fairly standard after rotating the velocity according to the interface normal. We prove a high order energy estimate for the non-linear equations that is independent of the artificial viscosity parameter which allows us to send it to zero. This approach loosely follows that introduced by Shkoller et al. in the setting of a compressible liquid-vacuum interface.

Although already considered by CoutAND et al. [10] and LindBLAD [17], we also make some brief comments on the case of a compressible liquid-vacuum interface, which is obtained from the vortex sheets problem by replacing one of the fluids by vacuum, where it is possible to obtain a structural stability result even without surface tension.
\end{abstract}

\section{Contents}

1. Introduction . . . . . . . . . . . . . . . . . . . 606

1.1. Description of a Vortex Sheet With or Without Surface Tension and its Stability . . . . . . . . . . . . . . . . . 606

1.2. Existing Results and Methodology for Similar Problems . . . . . . . . . . 608 
1.3. The Euler Equations for Inviscid Compressible Fluids . . . . . . . . . . . 609

1.3.1 Statement of the Equations and Jump Conditions . . . . . . . . . . 609

1.3.2 Entropy and a Symmetric Form of the Equations . . . . . . . . . 610

2. Notation . . . . . . . . . . . . . . . . . . . . . 611

3. The Main Theorem . . . . . . . . . . . . . . . . . . . . 613

3.1. Definition of Vortex Sheets with Surface Tension . . . . . . . . . . . 613

3.2. The Initial Data and the Compatibility Conditions . . . . . . . . . . . . . . . 614

3.3. The Energy Functional . . . . . . . . . . . . . . . . . . . . . . 615

3.4. Statement of the Main Theorem . . . . . . . . . . . . . . . . 616

4. Summary of the Proof of Theorem $1 \ldots \ldots$. . . . . . . . . . . 617

4.1. Fixing the Domains . . . . . . . . . . . . . . . . . . . 617

4.2. The $\mu$-Approximate Equations: A Degenerate Parabolic-Type Regularisation 617

4.3. The $\mu$-Independent Energy Estimate and Reduction to Solving the $\mu$-Approximate Equations . . . . . . . . . . . . . 618

4.4. Linearisation of the $\mu$-Approximate Equations . . . . . . . . . . . . . 618

4.5. Obtaining a Fixed Point of the Linearised Equations . . . . . . . . . . . 619

4.6. Proof of Uniqueness . . . . . . . . . . . . . . . . . . . . . . . . 619

5. The Theorem in the Fixed Domains _. . . . . . . . . . . . . . . . 619

5.1. Important Notational Convention . . . . . . . . . . . . . . . . . . 619

5.2. The Fixed Domains, the Lifting Operator and the Transformed Derivatives 620

5.3. Definition of the Equations in the Fixed Domains . . . . . . . . . . . 621

5.4. The Initial Data and the Compatibility Conditions . . . . . . . . . . . . . 622

5.5. The Energy Functional . . . . . . . . . . . . . . . . . . . . 623

5.6. Statement of the Theorem in the Fixed Domains . . . . . . . . . . . 623

6. Fixing the Domains . . . . . . . . . . . . . . . . . . . . . 625

6.1. The Change of Coordinates . . . . . . . . . . . . . . . . 625

6.2. Reduction to the Case of Fixed Domains . . . . . . . . . . . . . . . . . . 626

7. Smoothing the Initial Data . . . . . . . . . . . . . . . . . . . 627

7.1. Constructing Smooth Compatible Initial Data . . . . . . . . . . . . . . 627

7.2. Reduction to the Case of Smooth Initial Data . . . . . . . . . . . . . . . 630

8. The $\boldsymbol{\mu}$-Approximate Equations . . . . . . . . . . . . . . . . . 630

8.1. Definition of the $\mu$-Approximate Equations . . . . . . . . . . . . . . 630

8.2. Initial Data and the Compatibility Conditions . . . . . . . . . . . . . . 631

8.3. Energy for the $\mu$-Approximate Equations . . . . . . . . . . . . 632

8.4. Statement of Existence of Solutions to the $\mu$-Approximate Equations . . . 634

8.5. Statement of the $\mu$-Independent Energy Estimate for the $\mu$-Approximate Equations .............................. 635

8.6. Reduction to Solving the $\mu$-Approximate Equations . . . . . . . . . . 636

8.7. The Curl of the Velocity Equation . . . . . . . . . . . . . . . . . 638

9. Proof of the $\boldsymbol{\mu}$-Independent Energy Estimate for the $\mu$-Approximate Equations 639

9.1. Notation for Constants and Functions of the Energy and Initial Data . . . 639

9.2. The Material Derivative Operators . . . . . . . . . . . . . . . . . . . . 640

9.3. Restriction of the Time Interval . . . . . . . . . . . . . . . . . . 641

9.4. Using the Energy to Extend the Assumptions on the Initial Data . . . . . 641

9.5. Estimate of the Lower Order Terms in the Energy . . . . . . . . . . . . . 641

9.6. The Differentiated Equations and the Corrected Pressure, Velocity and Entropy . . . . . . . . . . . . . . . . . . 642

9.6 .1 The Differentiated Interior Equations . . . . . . . . . . . . . . . 642

9.6.2 The Corrected Pressure, Velocity and Entropy . . . . . . . . . . . 643

9.6.3 Tangential and Time Derivatives of the Jump Conditions . . . . . . 644

9.6 .4 Differentiating the Unit Normal . . . . . . . . . . . . . . . . . 645

9.7. A Slightly Lower Order Estimate for the Front . . . . . . . . . . . . . . . 645

9.8. Estimate of the Entropy . . . . . . . . . . . . . . . . . . . . . . . 646

9.9. Estimate of the Tangential Derivatives of the Pressure, Velocity and Front 646 
9.10. Estimate of the Time Derivatives of the Corrected Pressure and Velocity and the Front . . . . . . . . . . . . . . . . . . . 650

9.11. A Weighted Normal Derivative Estimate . . . . . . . . . . . . . . 658

9.12. Estimate of the Curl . . . . . . . . . . . . . . . . . . . . . . 659

9.13. Estimate of the Normal Derivatives of the Pressure and Velocity . . . . . 660

9.14. Estimate of the Artificial Viscosity Term . . . . . . . . . . . . . . . . . 664

9.15. Elliptic-Type Estimate for the Front . . . . . . . . . . . . . . . . . 664

9.16. Estimate of the Additional Terms in the Energy Involving $\mu \ldots \ldots$. . . . 666

9.17. Conclusion of the Energy Estimate . . . . . . . . . . . . . . . . . . . . 669

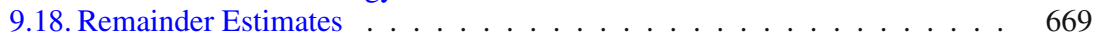

10. The Linearised Equations . . . . . . . . . . . . . . . . . . . . 673

10.1. Definition of the Linearised Equations . . . . . . . . . . . . . . . . . . . . 674

10.2. Definition of the Linearised Energy . . . . . . . . . . . . . . . . . 675

10.3. Notation for Constants and Functions of the Energy and Initial Data . . . 676

10.4. Statement of the Energy Estimate for the Linearised Equations . . . . . . 677

10.5. Statement of Existence and Uniqueness of Solutions to the Linearised Equations . . . . . . . . . . . . . . . . . 677

10.6. The Curl of the Linearised Velocity Equation _ . . . . . . . . . . . . 678

11. Solving the Linearised Equations for the Front, Entropy, Pressure and Curl . . . 679

12. Solving the Linearised Parabolic-Type Equation for the Velocity . . . . . . . . 681

12.1. Notation for Constants Depending on the Background State and the Solutions to Preceding Equations . . . . . . . . . . . . . . . . . 682

12.2. Statement of Existence and Uniqueness of Solutions to the Parabolic-Type Linearised Equation for the Velocity . . . . . . . . . . . . . . 682

12.3. Rotating the Velocity to Fix the Normal Jump Condition . . . . . . . . . 683

12.4. The Spaces for a Weak Form of the Equations . . . . . . . . . . . . . . . 685

12.5. Construction of a Galerkin Basis . . . . . . . . . . . . . . . . . 688

12.6. Existence, Uniqueness and Regularity Theory for Weak Equations of the Appropriate Form . . . . . . . . . . . . . . . . . . . . . 689

12.7. Proof of Existence, Uniqueness and Regularity of Solutions of the Equations for the Rotated Velocity . . . . . . . . . . . . . . . . . . . . . . 697

13. Proof of the Energy Estimate for the Linearised Equations _ . . . . . . . . . . 699

13.1. Lower Order Estimate . . . . . . . . . . . . . . . . . . . . . . . . . . . . 699

13.2. Estimate of the Front . . . . . . . . . . . . . . . . . 700

13.3. Estimate of the Entropy, Pressure and Curl . . . . . . . . . . . . . 700

13.4. Estimate of the Velocity . . . . . . . . . . . . . . . . . . . . . . . 704

13.5. Conclusion of the Energy Estimate . . . . . . . . . . . . . . . 708

14. The Fixed Point Scheme . . . . . . . . . . . . . . . . . . . . . . . . . 709

14.1. The Functional Framework . . . . . . . . . . . . . . . . . . . 709

14.2. The Contraction Mapping . . . . . . . . . . . . . . . . . . . 710

14.3. The Existence of a Solution to the $\mu$-Approximate Equations . . . . . . . 712

14.3.1 Conclusion of the Proof of the Existence of Solutions to the $\boldsymbol{\mu}$-Approximate Equations . . . . . . . . . . . . 716

14.3.2 Conclusion of the Proof of the Existence of Maximal-Time Solutions to the $\mu$-Approximate Equations _ . . . . . . . . . . 717

15. Proof of Uniqueness and Stability . . . . . . . . . . . . . . . . . 717

16. Extensions . . . . . . . . . . . . . . . . . . . . . . . . . . . . . 719

16.1. The Two-Dimensional Case . . . . . . . . . . . . . . . . . . . 719

16.2. Body Force . . . . . . . . . . . . . . . . . . . . . . . . . . 719

16.3. Horizontally Periodic Domain with Top and Bottom Walls . . . . . . . . 719

16.4. Bounded Domain . . . . . . . . . . . . . . . . . . . 720

17. The One-Phase Problem With and Without Surface Tension . . . . . . . . . . 721

17.1. Statement of the One-Phase Problem . . . . . . . . . . . . . . . 721

17.2. The One-Phase Problem With Surface Tension . . . . . . . . . . . . . . 722

17.3. The One-Phase Problem Without Surface Tension . . . . . . . . . . . . 723 
18. Appendix . . . . . . . . . . . . . . . . . . . . 727

18.1. A Hodge Decomposition Based Estimate . . . . . . . . . . . . . . . . . . 727

18.2. Products and Compositions in Sobolev Spaces . . . . . . . . . . . . . . 728

References . . . . . . . . . . . . . . . . . . . . . . . . . 729

\section{Introduction}

\subsection{Description of a Vortex Sheet With or Without Surface Tension and its Stability}

In this work, a vortex sheet refers to a virtual surface separating two inviscid fluids or two phases of the same inviscid fluid across which there is no fluid flow. We consider the three-dimensional vortex-sheet problem, which means that the fluids occupy three space dimensions and the surface is two-dimensional, but our results can easily be adapted to the two-dimensional problem. In general the tangential velocity is discontinuous across the surface, although we do not require this, and the density and entropy (depending on the equations of state satisfied by the two fluid phases) may jump. The fluid flow is modelled by the conversation of mass, momentum and energy, which leads to the use of the compressible Euler equations for the fluid either side of the surface. Conservation of momentum implies that the jump in pressure across the surface is not arbitrary, but must balance any surface force. Such structures are often seen in nature, such as when two currents of air at different temperatures meet in the atmosphere, or when oil is spilt on top of water. Vortex sheets can also develop in certain situations starting with smooth initial data for the Euler equations of gas dynamics.

We note that there are trivial vortex-sheet solutions to the Euler equations consisting of two constant states separated by a flat surface with zero normal velocity and zero pressure jump (Fig. 1). For the rest of this section we denote the velocity of these constant states by $\bar{u}_{+}$and $\bar{u}_{-}$, the density by $\bar{\rho}_{+}$and $\bar{\rho}_{-}$, and the sound speed by $\bar{c}_{+}$and $\bar{c}_{-}$. We are interested in whether such structures are stable, at least for a short time. In this work we do not require the initial data to be a perturbation of the flat vortex sheet described, but it is a good example to have in mind.

In the absence of a surface tension force which acts to flatten the surface of discontinuity, vortex sheets with a jump in tangential velocity are unstable in general. If the fluids are incompressible then we always have instability - this is the well-known Kelvin-Helmholtz instability. MiLEs [19] showed by normal modes analysis that compressible vortex sheets in two dimensions are linearly unstable unless the following stability criterion is satisfied:

$$
|[\bar{u}]| \geqq\left(\bar{c}_{+}^{\frac{2}{3}}+\bar{c}_{-}^{\frac{2}{3}}\right)^{\frac{3}{2}}
$$

where $[\bar{u}]=\bar{u}_{+}-\bar{u}_{-}$, under the simplifying assumption

$$
\bar{\rho}_{+} \bar{c}_{+}^{2}=\bar{\rho}_{-} \bar{c}_{-}^{2} .
$$

In this case they are linearly stable. In fact Coulombel and SECCHI [9] and [8] have shown using Nash-Moser iteration that (isentropic) vortex sheets in two dimensions 


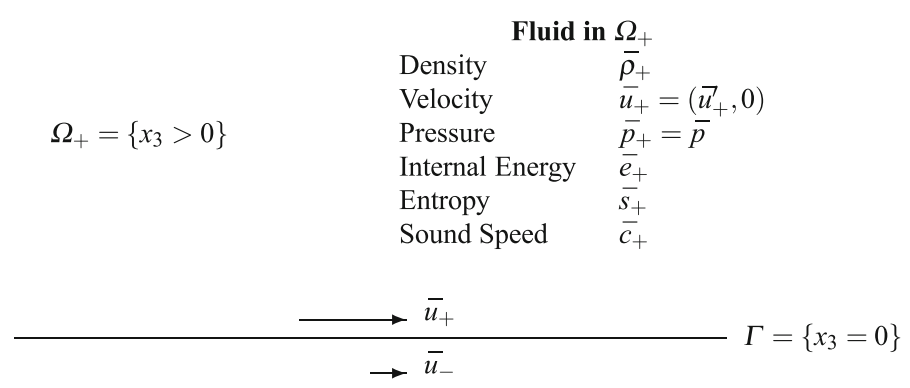

\begin{tabular}{lll} 
& \multicolumn{2}{c}{ Fluid in $\Omega_{-}$} \\
& Density & $\bar{\rho}_{-}$ \\
& Velocity & $\bar{u}_{-}=\left(\vec{u}_{-}, 0\right)$ \\
& Pressure & $\bar{p}_{-}=\bar{p}$ \\
& Internal Energy & $\bar{e}_{-}$ \\
& Entropy & $\bar{s}_{-}$ \\
& Sound Speed & $\bar{s}_{-}$
\end{tabular}

Fig. 1. Flat vortex sheet

are stable for a short time under this condition (as a strict inequality) if the initial perturbation of the flat vortex sheet is small enough in a high-order Sobolev norm. FEJER and MiLes [15] also showed, using a simple extension of the two dimensional argument, that vortex sheets in three dimensions are always linearly unstable.

Still in the absence of a surface tension, if the vortex sheet has no jump in tangential velocity initially, but the density jumps, then Rayleigh-Taylor instability will occur. Even if the Rayleigh-Taylor condition is satisfied (loosely speaking the pressure at the surface of discontinuity increases in the direction of the denser fluid), so that Rayleigh-Taylor instability does not occur, it seems that for general perturbations preserving the continuity of the velocity initially, the tangential velocity will immediately become discontinuous and then we will have the same instability as described above.

We therefore consider here vortex sheets in three dimensions with surface tension (Fig. 2), in the hope that surface tension will provide a stabilising effect. We assume that surface tension gives rise to a pressure force per unit area acting to flatten the surface which is proportional to the sum of the curvatures in two orthogonal directions, thus its strength is

$$
\sigma\left|\frac{1}{R_{1}}+\frac{1}{R_{2}}\right|=2 \sigma|H|=\sigma|\nabla \cdot \hat{n}|,
$$

where $R_{1}$ and $R_{2}$ are the principle radii of curvature of the surface, $H$ is the mean curvature of the surface, $\hat{n}$ is a unit normal to the surface and we call $\sigma$ the coefficient of surface tension, which has units of force per unit length. Note that although $\hat{n}$ is defined only on the surface, we compute $\nabla \cdot \hat{n}$ as $\nabla \cdot \hat{h}$, where $\hat{h}$ is any (sufficiently smooth) unit vector field on $\mathbb{R}^{3}$ which coincides with $\hat{n}$ on the surface, which one can check is independent of the choice of $\hat{h}$. In this work the coefficient of surface tension $\sigma>0$ will be a constant. 


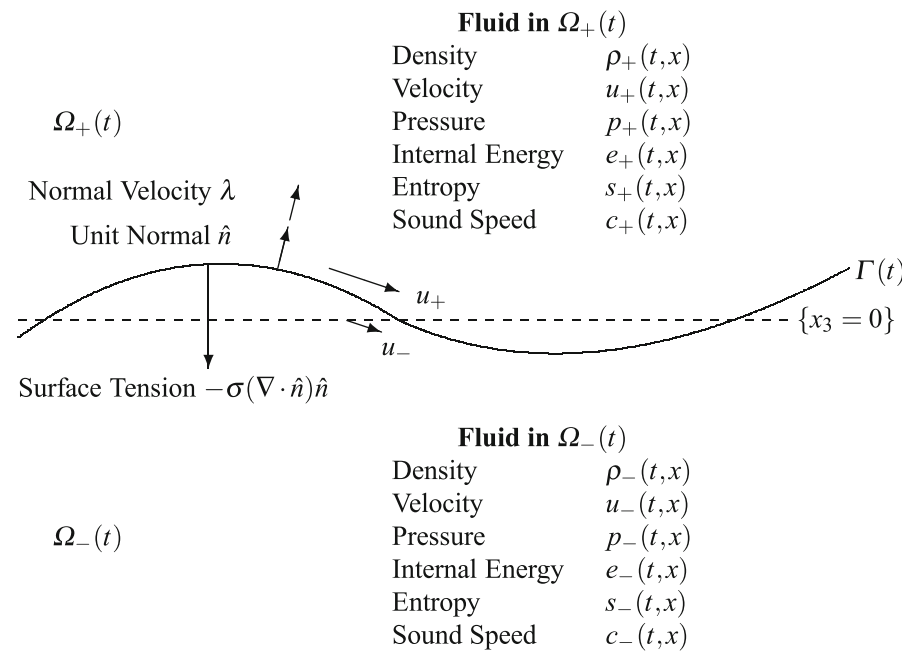

Fig. 2. Perturbation of a flat vortex sheet

\subsection{Existing Results and Methodology for Similar Problems}

The case of incompressible vortex sheets with surface tension has been studied by CHENG et al. [6], where they succeed in proving short-time structural stability. Their paper [6] is based on the methodology of the previous work of CoUTAND and SHKOLLER [12] and [11] for the incompressible liquid-vacuum interface problem. The difference here is that we consider the case of compressible vortex sheets with a very general pressure law. All we will need is that the sound speed is welldefined-that is $\left.\frac{\partial p}{\partial \rho}\right|_{s}>0$. We will also assume we are away from vacuum, that is the density is bounded below away from zero. We have already mentioned the short-time structural stability result proved by Coulombel and SECCHI [9] and [8] for compressible (isentropic) vortex sheets in two space dimensions without surface tension, which does not hold in three space dimensions as shown by the linear stability analysis of FEJER and MiLEs [15].

Another possible stabilisation effect on vortex sheets other than surface tension is the existence of a background magnetic field, assuming the fluid is electrically conducting, and this gives rise to current-vortex sheets in magnetohydrodynamics (MHD). Trakhinin [20] and CHEN and WANG [4,5] have succeeded in proving short-time existence of compressible current-vortex sheets under certain stability criteria involving the non-parallel jump in the magnetic field. Coulombel et al. [7] have obtained an a priori energy estimate for incompressible current-vortex sheets under equivalent stability criteria.

The one-phase problem (where one of the two phases is replaced by vacuum) has been solved for the case of an incompressible liquid by COUTAND and SHKOLLER [12] and [11] with and without surface tension and previously by LINDBLAD [18] without surface tension, and for the case of a compressible liquid by CoUTAND et al. [10] with and without surface tension and previously by LINDBLAD [17] without surface tension. Also, the case of a gas-vacuum boundary has been solved 
under certain boundary conditions by COUTAND and SHKOLLER [13] and JANG and MAsmoudi [16].

Note that standard approaches to hyperbolic PDEs such as the linearisation approach described in BENZONI et al. [3], or the addition of non-degenerate artificial viscosity as described in Evans [14], are not directly applicable to our problem as they destroy the structure of the equations on which our energy estimate relies.

Rather than follow the Nash-Moser scheme of $[4,9,17,20]$, which can often be very technical, we introduce a degenerate artificial viscosity type regularisation inspired by $[10,13,16]$, and prove a priori estimates on the nonlinear equations in a somewhat similar manner to [6], but using a change of coordinates which is a simple lift of the graph of the free-surface, similar to that of [7], rather than Lagrangian coordinates.

\subsection{The Euler Equations for Inviscid Compressible Fluids}

1.3.1. Statement of the Equations and Jump Conditions The Euler equations for compressible fluids are a system of PDEs for the macroscopic fluid variables pressure, $p$, density, $\rho$, specific internal energy, $e$ and velocity, $u$ as functions of time and space, which are written as follows:

$$
\begin{gathered}
\partial_{t} \rho+\nabla \cdot(\rho u)=0 \\
\partial_{t}(\rho u)+\nabla \cdot(\rho u \otimes u+p I)=0 \\
\partial_{t}\left(\rho e+\rho \frac{|u|^{2}}{2}\right)+\nabla \cdot\left(u\left(\rho e+\rho \frac{|u|^{2}}{2}+p\right)\right)=0 .
\end{gathered}
$$

Here, we have used the notation $\nabla \cdot$ for divergence, which is defined to act row-wise on matrices. The matrix $u \otimes u$ has $(i, j)$-entry $u_{i} u_{j}$ and $I$ is the identity matrix, so the $i$-entry of $\nabla \cdot(\rho u \otimes u+p I)$ is $\sum_{j=1}^{3} \partial_{x_{j}}\left(\rho u_{i} u_{j}\right)+\partial_{x_{i}} p$. They can be derived by considering conservation of mass, momentum and energy and neglecting viscous forces and heat conduction. In order to close the system of equations, we assume that the pressure is given by the equation of state $p=p(\rho, e)$.

The above equations should be satisfied in a classical sense where the fluid is sufficiently smooth. Now let us assume that the fluid is smooth either side of a surface of discontinuity $\Gamma(t)$. We will assume $\Gamma(t)$ is smooth with a well defined unit normal $\hat{n}(t, x)$, and moves with normal velocity $\lambda(t, x)$, for $x \in \Gamma(t)$. Since the above equations are written in divergence form, they may be interpreted in the weak sense in a region which contains the surface $\Gamma$ which means that we may derive jump conditions which must be satisfied across the surface of discontinuity without surface tension in order to have a weak solution in the entire region, which leads to the well-known Rankine-Hugoniot conditions. With surface tension present on the surface of discontinuity $\Gamma$ we must take into account the corresponding surface force produced, which gives rise to the following jump conditions:

$$
\begin{gathered}
{\left[m_{\hat{n}}\right]=0} \\
m_{\hat{n}}\left[u_{\hat{n}}\right]+[p]=-\sigma \nabla \cdot \hat{n} \\
m_{\hat{n}}\left[u_{\hat{\tau}}\right]=0
\end{gathered}
$$




$$
m_{\hat{n}}\left[\left(e+\frac{1}{2} u^{2}\right)+\frac{p}{\rho}\right]+\lambda[p]=-\sigma(\nabla \cdot \hat{n}) \lambda,
$$

where we have defined the mass flux $m_{\hat{n}}:=\rho(u \cdot \hat{n}-\lambda), u_{\hat{n}}=u \cdot \hat{n}$ is the normal component of $u, u_{\hat{\tau}}$ is the tangential part of $u$, and $[a]=a_{+}-a_{-}$denotes the jump in the quantity $a$ across $\Gamma(t)$.

For vortex sheets, where by definition $m_{\hat{n}}=0$, the above conditions simplify to the following jump conditions:

$$
\begin{aligned}
\left(u_{\hat{n}}\right)_{ \pm} & =\lambda \\
{[p] } & =-\sigma \nabla \cdot \hat{n} .
\end{aligned}
$$

1.3.2. Entropy and a Symmetric Form of the Equations The Euler equations as we have written them are not symmetric, which makes it impossible to obtain high-order energy estimates by differentiating the equations and integrating by parts. It turns out that the equations can be made symmetric in regions of smooth flow by a change of dependent variables involving the physical entropy.

The physical quantity, entropy per unit mass, $s$, can be defined as a function of $\rho$ and $e$ through the fundamental thermodynamic relation

$$
T \mathrm{~d} s=\mathrm{d} e+p \mathrm{~d} V=\mathrm{d} e-\frac{p}{\rho^{2}} \mathrm{~d} \rho
$$

where $V=\frac{1}{\rho}$ is the volume per unit mass and this equation also defines the state variable temperature $T=T(\rho, e)$, which must be strictly positive. Equivalently, we may take $s$ to be any smooth function $s(\rho, e)$ with $\left.\frac{\partial s}{\partial e}\right|_{\rho}>0$ for $\rho>0$ that satisfies the equation

$$
\left.\frac{\partial s}{\partial \rho}\right|_{e}+\left.\frac{p}{\rho^{2}} \frac{\partial s}{\partial e}\right|_{\rho}=0 .
$$

We now use the dependent variable $s$ instead of $e$ in the Euler equations. We also assume that $\left.\frac{\partial p}{\partial \rho}\right|_{s}>0$ so that we may write $\rho=\rho(p, s)$ and replace the dependent variable $\rho$ with $p$. It is easy to check assuming sufficient smoothness and using (4) that the equations become

$$
\begin{gathered}
\frac{1}{\rho c^{2}}\left(\partial_{t}+u \cdot \nabla\right) p+\nabla \cdot u=0 \\
\rho\left(\partial_{t}+u \cdot \nabla\right) u+\nabla p=0 \\
\left(\partial_{t}+u \cdot \nabla\right) s=0,
\end{gathered}
$$

where $c=\sqrt{\left.\frac{\partial p}{\partial \rho}\right|_{s}}>0$ is called the sound speed. This system is symmetric, and makes sense provided $\rho>0$ and $c>0$, which we will ensure throughout.

From the above discussion it should be clear that under the assumptions $\rho>0$, $c>0$ there is no loss of generality in considering classical solutions of the system just written instead of the original system (1)-(3). 
Let us now fix the equations of state

$$
\rho_{ \pm}=\rho_{ \pm}(p, s): \mathbb{R}^{2} \rightarrow \mathbb{R}
$$

for the density in the + and - regions as smooth functions of $p$ and $s$ in the region where $\rho_{ \pm}>0$. Similarly we define $c_{ \pm}=\sqrt{\left.\frac{\partial p_{ \pm}}{\partial \rho}\right|_{s_{ \pm}}}$and we assume $c_{ \pm}>0$ for $\rho_{ \pm}>0$.

\section{Notation}

Notation. Let $\Omega$ be an open set in $\mathbb{R}^{d}$ and let $k \geqq 0$. Then $C_{b}^{k}(\Omega)$ will denote the space of $k$-times continuously differentiable bounded functions on $\Omega$ with bounded derivatives up to order $k$. Note we write $C_{b}(\Omega):=C_{b}^{0}(\Omega)$.

We write $C_{c}^{k}(\Omega)$ for the space of $k$-times continuously differentiable functions on $\Omega$ with compact support in $\Omega$ and we write $C_{c}^{\infty}(\Omega)$ for the space of infinitely differentiable functions on $\Omega$ with compact support in $\Omega$.

Notation. We shall often need to refer to a quantity, say $a$, defined in two regions, denoted by + and - . We will write $a_{+}$for the quantity represented by $a$ in the + region and $a_{-}$for the quantity represented by $a$ in the - region. Sometimes for brevity we will use $a_{ \pm}$in formulae which hold for both $a_{+}$and $a_{-}$and sometimes for ease of reading we will omit the subscripts \pm altogether in such formulae, but the meaning should be clear from the context.

Notation. For a vector $w \in \mathbb{R}^{3}$, we will write $w^{\prime}$ for the first 2 components of $w$, so that $w=\left(w^{\prime}, w_{3}\right)$. For example, $x=\left(x^{\prime}, x_{3}\right)$, so that $x^{\prime}$ denotes horizontal position, $u=\left(u^{\prime}, u_{3}\right)$, so that $u^{\prime}$ denotes horizontal velocity, and $\nabla=\left(\nabla^{\prime}, \partial_{x_{3}}\right)$, so that $\nabla^{\prime}$ denotes horizontal gradient.

We will also write $\Delta^{\prime} a$ for the two-dimensional Laplacian $\partial_{x_{1}}^{2} a+\partial_{x_{2}}^{2} a$ for any function $a\left(x_{1}, x_{2}, x_{3}\right)$.

We will refer to the $x_{1}$ and $x_{2}$ directions as the horizontal or tangential directions and the $x_{3}$ direction as the vertical or normal direction. It will be important to distinguish these directions throughout what follows.

To avoid confusion, we will never use a prime' ${ }^{\prime}$ to denote a derivative.

Notation. Suppose $a \in L_{\text {loc }}^{1}\left((0, T) \times \mathbb{R}^{d}\right)$, where $d=2$ or $d=3$ in the main body of this work, has weak derivatives up to order $m$. Then we write

$$
\partial^{\alpha} a=\partial_{t}^{\alpha_{0}} \partial_{x_{1}}^{\alpha_{1}} \cdots \partial_{x_{d}}^{\alpha_{d}} a
$$

where $\alpha \in \mathbb{N}_{0}^{1+d}$ is a multi-index with $|\alpha| \leqq m$.

It is important to note that our definition of $\partial^{\alpha}$ includes time derivatives.

If $a \in L_{\mathrm{loc}}^{1}\left(\mathbb{R}^{d}\right)$ is a function of space only or if $a \in L_{\mathrm{loc}}^{1}\left((0, T) \times \mathbb{R}^{d}\right)$ as above, and has weak spatial derivatives up to order $m$, then we write

$$
\nabla^{\alpha} a=\partial_{x_{1}}^{\alpha_{1}} \cdots \partial_{x_{d}}^{\alpha_{d}} a
$$

where $\alpha \in \mathbb{N}_{0}^{d}$ is a multi-index with $|\alpha| \leqq m$. 
We also write

$$
\partial a=\left(\partial_{t} a, \partial_{x_{1}} a, \ldots, \partial_{x_{k}} a\right), \quad \nabla a=\left(\partial_{x_{1}} a, \ldots, \partial_{x_{k}} a\right) .
$$

Note that for convenience, we will sometimes write $\partial^{\alpha} a$ where $\alpha \in \mathbb{N}_{0}^{1+3}$ but $a \in L_{\text {loc }}^{1}\left((0, T) \times \mathbb{R}^{2}\right)$. Clearly in this case derivatives with respect to $x_{3}$ are zero. If there is a possibility of confusion, then we will either explicitly write $\alpha_{3}=0$ or use the notation $\alpha^{\prime}$ to denote a multi-index $\alpha \in \mathbb{N}_{0}^{1+2}$, so that $\partial^{\alpha^{\prime}}$ involves no derivatives with respect to $x_{3}$. We use the same convention for $\nabla^{\alpha} a$. Explicitly,

$$
\begin{aligned}
& \partial^{\alpha^{\prime}} a=\partial_{t}^{\alpha_{0}} \partial_{x_{1}}^{\alpha_{1}} \partial_{x_{2}}^{\alpha_{2}} a, \quad \nabla^{\alpha^{\prime}} a=\partial_{x_{1}}^{\alpha_{1}} \partial_{x_{2}}^{\alpha_{2}} a, \partial^{\prime} a=\left(\partial_{t} a, \partial_{x_{1}} a, \partial_{x_{2}} a\right), \\
& \nabla^{\prime} a=\left(\partial_{x_{1}} a, \partial_{x_{2}} a\right) .
\end{aligned}
$$

Sometimes we will also write $\partial^{l} a$ for the collection of all the time and space derivatives of $a$ of order $l$, and the product of this with another such quantity should be interpreted as sums of products of elements of the two quantities, and may be scalar or vector-valued as appropriate.

Notation. If we write $w \in X$ where $X$ usually denotes a space of scalar-valued functions, but $w=\left(w_{1}, \ldots, w_{k}\right)$ is a vector-valued function, then we mean that $w_{i} \in X$ for each $i$. Similarly, if we write $\partial^{\alpha} w$ then we mean $\left(\partial^{\alpha} w_{1}, \ldots, \partial^{\alpha} w_{k}\right)$.

Notation. Let $\Omega_{ \pm}$be two $C^{1}$ domains with common $C^{1}$ boundary $\Gamma$. If $a_{ \pm}$are functions defined on $\Omega_{ \pm}$which are uniformly continuous, and so can be defined on $\Gamma$ by continuity, write

$$
[a]=\left.a_{+}\right|_{\Gamma}-\left.a_{-}\right|_{\Gamma}
$$

If $a_{ \pm} \in H^{1}\left(\Omega_{ \pm}\right)$then we also use

$$
\left.a_{ \pm}\right|_{\Gamma}
$$

to denote the trace of $a_{ \pm}$on $\Gamma$ and we recall that

$$
\left\|\left.a_{ \pm}\right|_{\Gamma}\right\|_{H^{0.5}(\Gamma)} \leqq C\left\|a_{ \pm}\right\|_{H^{1}\left(\Omega_{ \pm}\right)} .
$$

See, for example, ADAMs and Fournier [1] for a proof.

It will be useful to have the following definition for smoothing later on.

Definition 1. For $d=2$ or 3 , let $\eta$ be the standard mollifier on $\mathbb{R}^{d}$. In particular $\eta \in C_{c}^{\infty}\left(\mathbb{R}^{d}\right)$ is supported in $\{|x| \leqq 1\}$ with $0 \leqq \eta \leqq C$ for some constant $C$ and we have $\int_{\mathbb{R}^{d}} \eta(x) \mathrm{d} x=1$. For $\varepsilon \in(0,1)$, set $\eta_{\varepsilon}(x)=\varepsilon^{-d} \eta\left(\frac{x}{\varepsilon}\right)$ so that $\eta_{\varepsilon} \in C_{c}^{\infty}\left(\mathbb{R}^{d}\right)$ is supported in $\{|x| \leqq \varepsilon\}$ with $0 \leqq \eta_{\varepsilon} \leqq C \varepsilon^{-d}$ and $\int_{\mathbb{R}^{d}} \eta_{\varepsilon}(x) \mathrm{d} x=1$.

It will also be useful to have the following definition of Sobolev extension. 
Definition 2. Let $\Omega \subset \mathbb{R}^{3}$ be a Lipschitz domain. We let $\operatorname{Ext}_{\Omega}: L_{\text {loc }}^{1}(\Omega) \rightarrow$ $L_{\text {loc }}^{1}\left(\mathbb{R}^{3}\right)$ denote a Sobolev extension operator such that

$$
\left\|\operatorname{Ext}_{\Omega} a\right\|_{W^{k, p}\left(\mathbb{R}^{3}\right)} \leqq C_{k}\|a\|_{W^{k, p}(\Omega)}
$$

for all $a \in L_{\text {loc }}^{1}(\Omega)$, all $p \in[1, \infty]$ and all $k \geqq 0$, where $C_{k}$ denotes a constant depending on $k$ and the constants of the Lipschitz domain $\Omega$. For example we could take Stein's extension operator. In fact we really only need the extension operator to satisfy the above inequality for $k$ up to a certain order-for example, $k \leqq 30$ would do, and we only need the case where $\Omega$ is a half-space.

\section{The Main Theorem}

\subsection{Definition of Vortex Sheets with Surface Tension}

Definition 3. Fix $T \in(0, \infty)$. Let $f \in C_{b}^{1}\left(\left(0, T^{\prime}\right) \times \mathbb{R}^{2}\right)$ with $\nabla^{\prime} f \in C_{b}^{1}\left(\left(0, T^{\prime}\right) \times\right.$ $\mathbb{R}^{2}$ ) for all $T^{\prime} \in(0, T)$, which we call the (graph of) the front or interface.

Define the moving domains $\Omega_{ \pm}(t)$ as

$$
\begin{aligned}
& \Omega_{+}(t)=\left\{x \in \mathbb{R}^{3}: x_{3}>f\left(t, x^{\prime}\right)\right\} \\
& \Omega_{-}(t)=\left\{x \in \mathbb{R}^{3}: x_{3}<f\left(t, x^{\prime}\right)\right\} .
\end{aligned}
$$

We also write

$$
\begin{aligned}
& \Omega_{+}^{T}=\left\{(t, x) \in(0, T) \times \mathbb{R}^{3}: x_{3}>f\left(t, x^{\prime}\right)\right\} \\
& \Omega_{-}^{T}=\left\{(t, x) \in(0, T) \times \mathbb{R}^{3}: x_{3}<f\left(t, x^{\prime}\right)\right\} .
\end{aligned}
$$

Define the moving boundary (or front or interface) $\Gamma(t)$ as

$$
\Gamma(t)=\left\{x \in \mathbb{R}^{3}: x_{3}=f\left(t, x^{\prime}\right)\right\} .
$$

We also write

$$
\Gamma^{T}=\left\{(t, x) \in(0, T) \times \mathbb{R}^{3}: x_{3}=f\left(t, x^{\prime}\right)\right\} .
$$

Define

$$
n:=\left(-\nabla^{\prime} f, 1\right) \text { and } \hat{n}:=\frac{n}{|n|}
$$

as a spatial normal and the spatial unit normal to the interface pointing into $\Omega_{+}(t)$. Note that since these are independent of $x_{3}$, we can think of them as being defined on $(0, T) \times \mathbb{R}^{2}$ or on $\Gamma^{T}$ via $n(t, x)=\left(-\nabla^{\prime} f\left(t, x^{\prime}\right), 1\right)$ for $(t, x) \in \Gamma^{T}$. Note also the normal velocity of the interface is given by $\frac{\partial_{t} f}{|n|}$.

Let $p_{ \pm},\left(u_{i}\right)_{ \pm}(i=1,2,3), s_{ \pm} \in C_{b}^{1}\left(\Omega_{ \pm}^{T^{\prime}}\right)$ for all $T^{\prime} \in(0, T)$. Write the vectors $u_{ \pm}=\left(\left(u_{1}\right)_{ \pm},\left(u_{2}\right)_{ \pm},\left(u_{3}\right)_{ \pm}\right)$and $U_{ \pm}=\left(p_{ \pm}, u_{ \pm}, s_{ \pm}\right)$. Note that $\left.U_{ \pm}\right|_{t=0}$ and $\left.f\right|_{t=0}$ are defined by uniform continuity.

We define $\rho_{ \pm}(t, x):=\rho_{ \pm}\left(p_{ \pm}(t, x), s_{ \pm}(t, x)\right)$ and $c_{ \pm}(t, x):=c_{ \pm}\left(p_{ \pm}(t, x)\right.$, $\left.s_{ \pm}(t, x)\right)$, where $\rho_{ \pm}(p, s)$ and $c_{ \pm}(p, s)$ are the equations of state defined in (8). 
We will say that $\left(U_{+}, U_{-}, f\right)$ is a vortex sheet solution of the Euler equations with surface tension on the time interval $(0, T)$ with initial data $\left(U_{+}^{0}, U_{-}^{0}, f^{0}\right):=$ $\left(\left.U_{+}\right|_{t=0},\left.U_{-}\right|_{t=0},\left.f\right|_{t=0}\right)$ provided that $\rho_{ \pm}(t, x)>0$ in $\Omega_{ \pm}^{T}$, and $p_{ \pm}, u_{ \pm}, s_{ \pm}, f$ satisfy the following system of PDEs.

$$
\begin{gathered}
\frac{1}{\rho c^{2}}\left(\partial_{t}+u \cdot \nabla\right) p+\nabla \cdot u=0 \\
\rho\left(\partial_{t}+u \cdot \nabla\right) u+\nabla p=0 \\
\left(\partial_{t}+u \cdot \nabla\right) s=0
\end{gathered}
$$

in $\Omega_{ \pm}^{T}$

$$
\begin{gathered}
\partial_{t} f=u_{ \pm} \cdot n \\
{[p]=-\sigma \nabla^{\prime} \cdot \hat{n}}
\end{gathered}
$$

on $\Gamma^{T}$.

\subsection{The Initial Data and the Compatibility Conditions}

Definition 4. We say that $\left(p_{ \pm}^{0}, u_{ \pm}^{0}, s_{ \pm}^{0}, f^{0}\right)$ are initial data for the system (9)-(13) if the following holds. We require that $f^{0} \in C_{b}^{1}\left(\mathbb{R}^{2}\right)$. We define the initial domains $\Omega_{ \pm}^{0}$, the initial boundary $\Gamma^{0}$ and the initial normal $n^{0}$ and unit normal $\hat{n}^{0}$ in the obvious way, replacing $f\left(t, x^{\prime}\right)$ in Definition 3 by $f^{0}\left(x^{\prime}\right)$.

We also require that $p_{ \pm}^{0}, u_{ \pm}^{0}, s_{ \pm}^{0} \in C_{b}\left(\Omega_{ \pm}^{0}\right)$ are uniformly continuous functions (where $u_{ \pm}^{0}$ are vectors representing the initial velocity), so that we may define them on the interface $\Gamma^{0}$ by uniform continuity. Finally, we require

$$
\rho_{ \pm}^{0}(x):=\rho_{ \pm}\left(p_{ \pm}^{0}(x), s_{ \pm}^{0}(x)\right)>0
$$

for all $x \in \Omega_{ \pm}^{0}$.

Definition 5. For integer $j \geqq 0$, we define the following differential operators $\partial_{0}^{j}$ associated with the system (9)-(13) which act on differentiable expressions involving the initial data and its derivatives (including compositions). We call the operator $\partial_{0}^{j}$ the initial time derivative operator of order $j$ associated to the system (9)-(13).

To start with, we define $\partial_{0}^{j} p_{ \pm}^{0}, \partial_{0}^{j} u_{ \pm}^{0}, \partial_{0}^{j} s_{ \pm}^{0}, \partial_{0}^{j} f^{0}$ as follows. We set

$$
\partial_{0}^{0}=\mathrm{id}
$$

then for $j \geqq 0$ we inductively define

$$
\begin{aligned}
& \partial_{0}^{j+1} f^{0}:=\left.\partial_{t}^{j}\left(\left.u_{+}(t)\right|_{\Gamma(t)} \cdot n(t)\right)\right|_{t=0}:=\left.\partial_{t}^{j}\left(u_{+}\left(t, x^{\prime}, f\left(t, x^{\prime}\right)\right) \cdot n(t)\right)\right|_{t=0} \\
& \partial_{0}^{j+1} p_{ \pm}^{0}:=\left.\partial_{t}^{j}\left(-\left(u_{ \pm}(t) \cdot \nabla\right) p_{ \pm}(t)-\rho_{ \pm}(t) c_{ \pm}^{2}(t) \nabla \cdot u_{ \pm}(t)\right)\right|_{t=0} \\
& \partial_{0}^{j+1} u_{ \pm}^{0}:=\left.\partial_{t}^{j}\left(-\left(u_{ \pm}(t) \cdot \nabla\right) u_{ \pm}(t)-\frac{1}{\rho_{ \pm}(t)} \nabla p_{ \pm}(t)\right)\right|_{t=0}
\end{aligned}
$$




$$
\partial_{0}^{j+1} s_{ \pm}^{0}:=\left.\partial_{t}^{j}\left(-\left(u_{ \pm}(t) \cdot \nabla\right) s_{ \pm}(t)\right)\right|_{t=0}
$$

where the right hand side means we think of $u_{ \pm}(t)$ etc. as functions of $t$ and formally differentiate with respect to $t j$ times according to the chain and Leibniz rules, then replace $\partial_{t}^{l} u_{ \pm}(t)$ for $0 \leqq l \leqq j$ by $\partial_{0}^{l} u_{ \pm}^{0}$ etc. Note that it is necessary for us to pick either + or - in the above definition for $\partial_{0}^{j+1} f^{0}$ since we have not yet imposed any compatibility conditions on the initial data. Note we require sufficient regularity on the initial data for the above to make sense.

We can now generalise the operators $\partial_{0}^{j}$ to general differentiable expressions involving the initial data and its derivatives (including compositions) in the obvious way. Suppose $H\left(p_{ \pm}^{0}, u_{ \pm}^{0}, s_{ \pm}^{0}, f^{0}\right)$ is such an expression. For $j \geqq 0$, we set

$$
\partial_{0}^{j}\left(H\left(p_{ \pm}^{0}, u_{ \pm}^{0}, s_{ \pm}^{0}, f^{0}\right)\right):=\left.\partial_{t}^{j}\left(H\left(p_{ \pm}(t), u_{ \pm}(t), s_{ \pm}(t), f(t)\right)\right)\right|_{t=0}
$$

where, as above, the right hand side means we think of $u_{ \pm}(t)$ etc as functions of $t$ and formally differentiate with respect to $t j$ times according to the chain and Leibniz rules, then replace $\partial_{t}^{l} u_{ \pm}(t)$ for $0 \leqq l \leqq j$ by $\partial_{0}^{l} u_{ \pm}^{0}$, etc.

Definition 6. Let $\left(p_{ \pm}^{0}, u_{ \pm}^{0}, s_{ \pm}^{0}, f^{0}\right)$ be initial data for the system (9)-(13). We say that the initial data $\left(p_{ \pm}^{0}, u_{ \pm}^{0}, s_{ \pm}^{0}, f^{0}\right)$ satisfy the compatibility conditions for the system (9)-(13) up to order $k$, for integer $k \geqq 0$, if the following holds for all $x^{\prime} \in \mathbb{R}^{2}$ :

$$
\partial_{0}^{j}\left[\left.u^{0}\right|_{\Gamma^{0}}\left(x^{\prime}\right) \cdot n^{0}\left(x^{\prime}\right)\right]=0
$$

for $0 \leqq j \leqq k$ and

$$
\left[\partial_{0}^{j}\left(\left.p^{0}\right|_{\Gamma^{0}}\left(x^{\prime}\right)\right)\right]=-\sigma \partial_{0}^{j}\left(\nabla^{\prime} \cdot \hat{n}^{0}\right)\left(x^{\prime}\right)
$$

for $0 \leqq j \leqq k$, where

$$
\begin{aligned}
& \left.u^{0}\right|_{\Gamma^{0}}\left(x^{\prime}\right):=u^{0}\left(x^{\prime}, f^{0}\left(x^{\prime}\right)\right) \\
& \left.p^{0}\right|_{\Gamma^{0}}\left(x^{\prime}\right):=p^{0}\left(x^{\prime}, f^{0}\left(x^{\prime}\right)\right) .
\end{aligned}
$$

\subsection{The Energy Functional}

Definition 7. Let $f \in L_{\text {loc }}^{1}\left((0, T) \times \mathbb{R}^{2}\right), U_{ \pm} \in L_{\text {loc }}^{1}\left(\Omega_{ \pm}^{T}\right)$. We define the energy $E$ for the vortex sheet equations, associated to $\left(U_{+}, U_{-}, f\right)$, as follows.

Define the energy $E:(0, T] \rightarrow[0, \infty]$ by

$$
\begin{aligned}
& E(t)=\sum_{ \pm} \operatorname{ess~sup}_{\tau \in(0, t)}\left\|U_{ \pm}\right\|_{L^{\infty}\left(\Omega_{ \pm}(\tau)\right)}^{2}+\sum_{ \pm} \sum_{1 \leqq|\alpha| \leqq 3} \operatorname{ess} \sup \left\|\partial^{\alpha} U_{ \pm}\right\|_{L^{2}\left(\Omega_{ \pm}(\tau)\right)}^{2} \\
& +\underset{\tau \in(0, t)}{\operatorname{ess} \sup \|f\|_{L^{\infty}\left(\mathbb{R}^{2}\right)}^{2}}+\sum_{1 \leqq\left|\alpha^{\prime}\right| \leqq 3} \operatorname{ess} \sup \left\|\partial^{\alpha^{\prime}} f\right\|_{H^{1}\left(\mathbb{R}^{2}\right)}^{2}
\end{aligned}
$$

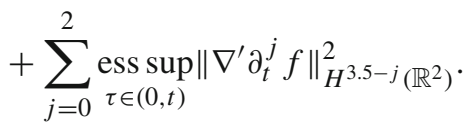


Note that we adopt the convention that if the solution does not have sufficiently many weak derivatives in $\Omega_{ \pm}^{T}$ to define the energy then it is infinite, and if we write $E\left(T^{\prime}\right)<\infty$ then this implies that all the weak derivatives in $\Omega_{ \pm}^{T^{\prime}}$ present in the energy exist. Note also that if $E\left(T^{\prime}\right)<\infty$ then $E$ is uniformly continuous on $\left(0, T^{\prime}\right]$.

Similarly, let $\left(U_{+}^{0}, U_{-}^{0}, f^{0}\right)$ be initial data for the system (9)-(13). We define the energy $E^{0} \in[0, \infty]$ of the initial data as

$$
\begin{aligned}
E^{0}= & \sum_{ \pm}\left\|U_{ \pm}^{0}\right\|_{L^{\infty}\left(\Omega_{ \pm}^{0}\right)}^{2}+\sum_{ \pm} \sum_{1 \leqq|\beta|+j \leqq 3}\left\|\nabla^{\beta} \partial_{0}^{j} U_{ \pm}^{0}\right\|_{L^{2}\left(\Omega_{ \pm}^{0}\right)}^{2} \\
& +\left\|f^{0}\right\|_{L^{\infty}\left(\mathbb{R}^{2}\right)}^{2}+\sum_{1 \leqq\left|\beta^{\prime}\right|+j \leqq 3}\left\|\nabla^{\beta^{\prime}} \partial_{0}^{j} f^{0}\right\|_{H^{1}\left(\mathbb{R}^{2}\right)}^{2}+\sum_{j=0}^{2}\left\|\nabla^{\prime} \partial_{0}^{j} f^{0}\right\|_{H^{3.5-j}\left(\mathbb{R}^{2}\right)}^{2}
\end{aligned}
$$

Note that we may consider the class of initial data such that $E^{0}<\infty$, which has the obvious meaning that $U^{0} \in L^{\infty}\left(\Omega_{ \pm}^{0}\right), f^{0} \in L^{\infty}\left(\Gamma^{0}\right), \nabla U^{0} \in H^{2}\left(\Omega_{ \pm}^{0}\right)$, $\nabla^{\prime} f^{0} \in H^{3.5}\left(\Gamma^{0}\right)$, which allows us to define $\partial_{0}^{j}\left(U_{+}^{0}, U_{-}^{0}, f^{0}\right)$ for $0 \leqq j \leqq 3$ using Definition 5, and we then require $\partial_{0}^{j} f^{0} \in H^{4-j}\left(\Gamma^{0}\right)$ for $1 \leqq j \leqq 3$ and $\partial_{0}^{j} f^{0} \in H^{4.5-j}\left(\Gamma^{0}\right)$ for $1 \leqq j \leqq 2$. (Note that the required regularity of $\partial_{0}^{j} U_{ \pm}^{0}$ for $1 \leqq j \leqq 3$ automatically follows from Definition 5 and the spatial regularity).

\subsection{Statement of the Main Theorem}

Theorem 1. Let $\left(U_{+}^{0}, U_{-}^{0}, f^{0}\right)$ be initial data as described in Definition 4 with energy $E^{0}<\infty$. Assume that this initial data satisfies the compatibility conditions (18)-(19) up to order 2, and note that these conditions make sense up to order 2 since we have $E^{0}<\infty$. Assume also that the initial density satisfies

$$
\inf _{x \in \Omega_{ \pm}^{0}} \rho_{ \pm}^{0}(x)=: \delta^{0}>0 .
$$

Then there exists a time $T^{0}>0$ and a vortex sheet solution $\left(U_{+}, U_{-}, f\right)$ of the Euler equations with surface tension on the time interval $\left(0, T^{0}\right)$, as in Definition 3. Moreover,

$$
E\left(T^{0}\right) \leqq C^{0}, \quad \rho_{ \pm} \geqq \frac{\delta^{0}}{2} \quad \text { in } \quad \Omega_{ \pm}^{T^{0}}
$$

The time $T^{0}>0$ is bounded below as follows:

$$
T^{0} \geqq T\left(E^{0}, \frac{1}{\delta^{0}}\right)>0,
$$

where $T(\cdot)$ is a smooth decreasing function. Similarly, the constant $C^{0}>0$ is bounded above as follows:

$$
C^{0} \leqq C\left(E^{0}, \frac{1}{\delta^{0}}\right)<\infty,
$$

where $C(\cdot)$ is a smooth increasing function. 
In addition, the solution $\left(U_{+}, U_{-}, f\right)$ is unique on the time interval $\left(0, T^{0}\right)$ in the sense that it is the only solution of the equations given in Definition 3 on the time interval $\left(0, T^{0}\right)$ with initial data $\left(U_{+}^{0}, U_{-}^{0}, f^{0}\right)$ satisfying the following properties

$$
E\left(T^{0}\right)<\infty, \quad \inf _{(t, x) \in \Omega_{ \pm}^{T^{0}}} \rho_{ \pm}>0 .
$$

Proof. See Sections 4-15.

Remark 1. In fact a statement of stability also holds in addition to uniqueness, but this is more easily stated after fixing the domains, so we leave this to Theorem 2.

\section{Summary of the Proof of Theorem 1}

\subsection{Fixing the Domains}

We need to introduce a change of coordinates on the whole space which maps the unknown domains $\Omega_{ \pm}^{T}$ separated by $\Gamma^{T}$ to/from some fixed domains $(0, T) \times \Omega_{ \pm}$ separated by a fixed boundary $(0, T) \times \Gamma$. We could use Lagrangian coordinates on one side of the interface with a Sobolev extension to the other side (which is forced by the discontinuity in the velocity), as used in [6], but this has the disadvantage of producing a different set of equations in $\Omega_{+}$and $\Omega_{-}$. We use the more straightforward approach of constructing a lift $\psi$ of $f$ defined on $(0, T) \times \Omega_{ \pm}$ such that $\left.\psi\right|_{\Gamma}=f$, where $\Omega_{+}=\mathbb{R}_{>0}^{3}, \Omega_{-}=\mathbb{R}_{<0}^{3}$ and $\Gamma=\left\{x \in \mathbb{R}^{3}: x_{3}=0\right\}=$ $\mathbb{R}^{2}$. We then use the change of coordinates mapping back to the original domains $(t, x) \mapsto\left(t, x^{\prime}, x_{3}+\psi(t, x)\right)$. To construct $\psi$, we merely multiply $f$ by a smooth cut-off function depending on $x_{3}$. Compared to constructing a lift of $f$ that gains half a degree of regularity from $f$ as used in [7], this has the advantage that $\psi$ is smooth in the $x_{3}$-direction, and we do not need the extra half a degree of regularity due to the presence of surface tension.

\subsection{The $\mu$-Approximate Equations: A Degenerate Parabolic-Type Regularisation}

We introduce some degenerate artificial viscosity inspired by $[10,13,16]$ in order to regularise the equations whilst preserving a good energy estimate.

We define the $\mu$-approximate equations (39)-(43) in Section 8 which reduce to the equations in the fixed domains (25)-(29) in the case $\mu=0$. There are four main points to note. Firstly, we regularise the divergence of the velocity by adding the term $\mu \nabla^{\psi}\left(\rho c^{2} \nabla^{\psi} \cdot u\right)$ to the right hand side of the velocity equation. This preserves the curl estimate, and it also preserves our estimate of $\partial_{x_{3}} p$ (which comes from writing it in terms of the material derivative of the pressure). This forces us to replace $[p]$ with $\left[p-\mu \rho c^{2} \nabla^{\psi} \cdot u\right]$ in the pressure interface condition (29). Secondly, we regularise $u \cdot n=\partial_{t} f$ by adding the term $\mu\left(\Delta^{\prime}-1\right)(u \cdot n)$ to the right hand side of the pressure interface condition (29), which overcomes the fact that surface tension only regularises $f$ in space not in time. Thirdly, we smooth the coefficient $\rho$ in the velocity equation (26), which is fairly harmless but allows 
us to overcome a difficulty in the curl equation when solving the $\mu$-approximate equations. Fourthly, we add some known smooth functions $g_{1}$ and $g_{2}$ to the right hand side of the velocity equation and pressure interface condition respectively in order to preserve the compatibility conditions.

\subsection{The $\mu$-Independent Energy Estimate and Reduction to Solving the $\mu$-Approximate Equations}

The key to the whole result is to prove that some high-order energy associated to solutions of the $\mu$-approximate equations, which we will call the $\mu$-independent energy, remains bounded independent of $\mu$ on some time interval which has a lower bound independent of $\mu$, provided that the solution exists for that length of time.

The $\mu$-independent energy is chosen to include roughly space and time derivatives of the solution up to order $3, L^{2}$ in space and $L^{\infty}$ in time. In addition there is one extra space derivative on $f$ with up to 3 time derivatives, and 1.5 extra space derivatives on $f$ with up to 2 time derivatives, which is an automatic consequence of the elliptic-type equation for $f$ given by the surface tension term in the pressure interface condition (13). We need 3 derivatives on the solution in order to be able to estimate terms involving one derivative of the solution in $L^{\infty}$ using the Sobolev embedding theorem.

The energy estimate is split into several parts which we briefly describe as follows. Firstly, we estimate the tangential space derivatives of the solution by differentiating the equations and performing a standard energy estimate. The boundary term generated may be estimated by applying tangential derivatives to the boundary conditions and using the extra spatial regularity of $f$ provided by surface tension. Next, we estimate the time derivatives of the solution in a similar manner, the major difference being the treatment of the boundary term, which we must split into two parts, one of which is estimated by converting it to an integral over the interior, and one of which we can estimate using the boundary conditions. Finally, we estimate the normal derivatives by taking the curl of the velocity equation and performing a standard energy estimate then obtaining estimates for the divergence of the velocity and the normal derivative of the pressure by directly rearranging the pressure and velocity equations. The high-order space derivative estimate of $f$ is obtained from the elliptic-like pressure interface condition.

\subsection{Linearisation of the $\mu$-Approximate Equations}

We have designed the $\mu$-approximate equations so that for fixed $\mu$ the equations have a nice linearised structure when linearised carefully. In fact the linearisation reduces to solving the following equations in order, which are given in Section 10. Firstly, we solve the very simple ODE $\partial_{t} f=\bar{u} \cdot \bar{n}$ for $f$. Next, we solve scalar linear transport equations where the transport is parallel to the interface for the entropy, the pressure, and (the components of) the curl, $\omega$. We carefully replace $u^{\psi}$ with $\overline{u^{\psi}}$ (see Section 10 for the definition) which is chosen so that $\left.\bar{u}^{\psi}{ }_{3}\right|_{\Gamma}=\bar{u} \cdot \bar{n}-\partial_{t} f=0$ by 
virtue of the equation just given for $f$. Finally, we solve a parabolic-type equation for the velocity, where the full parabolicity comes from the fact that we may add the curl of the curl of the velocity to one side of the equation and the curl of the 'curl' $\omega$ just obtained from the transport equation for the curl, $\omega$, to the other side of the equation.

The transport equations are standard, but the solution of the parabolic-type equation for the velocity requires some work and is detailed in Section 12. Note that we solve the equation in the domains $\Omega_{ \pm}$simultaneously whilst maintaining the boundary condition $[u \cdot n]=0$. It seems very difficult to preserve an appropriate energy estimate without maintaining the condition $[u \cdot n]=0$, which contrasts with the incompressible case considered in [6]. Also, note that in order to be able to pose the equations in standard function spaces we first rotate the velocity to obtain $\tilde{u}$ so that the condition $[u \cdot n]=0$ becomes $\left[\tilde{u}_{3}\right]=0$, after which we proceed much as in the solution of a simple linear parabolic equation.

\subsection{Obtaining a Fixed Point of the Linearised Equations}

In Section 14 we use the contraction mapping theorem to prove the existence of a fixed point of the linearised equations, which is a solution of the $\mu$-approximate equations by construction. This is fairly standard once we have obtained the appropriate energy estimate for the linearised equations, given in Proposition 11 and proved in Section 13. This completes the proof of the existence part of Theorem 1.

\subsection{Proof of Uniqueness}

The proof of uniqueness is very similar to the $\mu$-independent energy estimate with $\mu$ set to zero. Given two solutions satisfying the conclusions of the existence part of theorem on the fixed domains, Theorem 2, we define the 'difference' energy by replacing the solution by the difference of two solutions in the terms which make up the energy for the fixed equations as defined in Definition 15, and decreasing the number of derivatives by one. We then show that this energy is bounded by a factor depending on the individual energies of the solutions as defined by Definition 15 multiplying the sum of the difference energy at time zero and a time integral of the difference energy. Applying Gronwall's lemma gives a stability result for the equations in the fixed domains, from which uniqueness follows.

\section{The Theorem in the Fixed Domains}

In this section we present the equations we obtain from the system (9)-(13) rewritten on fixed domains after a change of variables, which is detailed in Section 6.

\subsection{Important Notational Convention}

Everything that follows after Section 6 will be devoted to the proof of the theorem in the fixed domains. Hence, to keep the notation simple, we will now 
redefine everything in the fixed domains, which will involve using some of the same symbols as in the moving domains for corresponding quantities, such as $\left(U_{+}, U_{-}, f\right)$ for the solution, $E$ for the energy, $\partial_{0}^{j}$ for the initial time derivatives associated with the system etc. In Section 6, where we show how the theorem in the fixed domains implies the main theorem, we will place a tilde, , over variables in the fixed domains in order to distinguish corresponding quantities, but otherwise we will reserve the tilde, , for other purposes.

\subsection{The Fixed Domains, the Lifting Operator and the Transformed Derivatives}

Definition 8. Define the fixed domains $\Omega_{ \pm}$as

$$
\begin{aligned}
& \Omega_{+}=\left\{x \in \mathbb{R}^{3}: x_{3}>0\right\} \\
& \Omega_{-}=\left\{x \in \mathbb{R}^{3}: x_{3}<0\right\} .
\end{aligned}
$$

Define the fixed boundary (or front or interface) $\Gamma$ as

$$
\Gamma=\left\{x \in \mathbb{R}^{3}: x_{3}=0\right\}
$$

Definition 9. Let $a \in \mathbb{R}_{\geqq 0}$. We define the lifting operator $L^{a}: L^{2}\left(\mathbb{R}^{2}\right) \rightarrow L^{2}\left(\mathbb{R}^{3}\right)$ by

$$
\left(L^{a} f\right)(x):=\chi\left(\frac{x_{3}}{3(1+a)}\right) f\left(x^{\prime}\right)
$$

where $\chi \in C_{c}^{\infty}(\mathbb{R})$ is a smooth cut-off function with $0 \leqq \chi \leqq 1, \chi\left(x_{3}\right)=1$ for $\left|x_{3}\right| \leqq 1, \chi\left(x_{3}\right)=0$ for $\left|x_{3}\right| \geqq 3$ and $\left|\partial_{x_{3}} \chi\left(x_{3}\right)\right| \leqq 1$ for all $x_{3} \in \mathbb{R}$.

Note that this implies the following.

$$
\begin{gathered}
\left(L^{a} f\right)\left(x^{\prime}, 0\right)=f\left(x^{\prime}\right) \\
\partial_{x_{3}}\left(L^{a} f\right)\left(x^{\prime}, 0\right)=0 \\
\left|\partial_{x_{3}}\left(L^{a} f\right)(x)\right| \leqq \frac{1}{3(1+a)}\left|f\left(x^{\prime}\right)\right| .
\end{gathered}
$$
by

Clearly for $T>0$ we may also define $L^{a}: L^{2}\left((0, T) \times \mathbb{R}^{2}\right) \rightarrow L^{2}\left((0, T) \times \mathbb{R}^{3}\right)$

$$
\left(L^{a} f\right)(t, x):=\chi\left(\frac{x_{3}}{3(1+a)}\right) f\left(t, x^{\prime}\right) .
$$

Note that $L^{a} f$ inherits the regularity of $f$ and derivatives of $L^{a} f$ can be bounded by those of $f$ independently of $a$. This means that all constants will be independent of $a$ unless explicitly stated otherwise. 
Definition 10. Given $\psi \in C^{1}\left((0, T) \times \mathbb{R}^{3}\right)$ bounded with bounded derivatives, and a vector $u \in \mathbb{R}^{3}$ (which we later take as a function of $t$ and $x$ ), we define

$$
\begin{gathered}
J^{\psi}:=1+\partial_{x_{3}} \psi \\
\partial^{\psi}:=\partial-\frac{1}{J^{\psi}} \partial \psi \partial_{x_{3}} \\
\nabla^{\psi}:=\nabla-\frac{1}{J^{\psi}} \nabla \psi \partial_{x_{3}}=\nabla^{\prime}+\frac{1}{J^{\psi}}\left(-\nabla^{\prime} \psi, 1\right) \partial_{x_{3}} \\
u^{\psi}:=\left(u^{\prime}, \frac{1}{J^{\psi}}\left(u_{3}-u^{\prime} \cdot \nabla^{\prime} \psi-\partial_{t} \psi\right)\right) .
\end{gathered}
$$

The quantity $J^{\psi}$ will be the Jacobian of the change of coordinates introduced later and $\partial$ acting on the fluid variables in the moving domains will transform to $\partial^{\psi}$ acting on the new fluid variables in the fixed domains after the change of coordinates introduced later-see Lemma 1.

\subsection{Definition of the Equations in the Fixed Domains}

Definition 11. Fix $T \in(0, \infty)$.

Let $f \in C_{b}^{1}\left(\left(0, T^{\prime}\right) \times \mathbb{R}^{2}\right)$ with $\nabla^{\prime} f \in C_{b}^{1}\left(\left(0, T^{\prime}\right) \times \mathbb{R}^{2}\right)$ for all $T^{\prime} \in(0, T)$, which we call the (graph of) the front or interface (although it is not the graph of the fixed interface $\Gamma=\mathbb{R}^{2}$ ). Define

$$
n:=\left(-\nabla^{\prime} f, 1\right) \text { and } \hat{n}:=\frac{n}{|n|},
$$

which we call a spatial normal and the spatial unit normal to the interface pointing into $\Omega_{+}$(although they are not actually normals to the fixed interface $\Gamma=\mathbb{R}^{2}$ ).

Let $p_{ \pm},\left(u_{i}\right)_{ \pm}(i=1,2,3), s_{ \pm} \in C_{b}^{1}\left(\left(0, T^{\prime}\right) \times \Omega_{ \pm}\right)$for all $T^{\prime} \in(0, T)$. For convenience we write $u_{ \pm}=\left(\left(u_{1}\right)_{ \pm},\left(u_{2}\right)_{ \pm},\left(u_{3}\right)_{ \pm}\right), U_{ \pm}=\left(p_{ \pm}, u_{ \pm}, s_{ \pm}\right)$. Note that $\left.U_{ \pm}\right|_{t=0}$ and $\left.f\right|_{t=0}$ are defined by uniform continuity.

We define $\rho_{ \pm}(t, x):=\rho_{ \pm}\left(p_{ \pm}(t, x), s_{ \pm}(t, x)\right)$ and $c_{ \pm}(t, x):=c_{ \pm}\left(p_{ \pm}(t, x)\right.$, $\left.s_{ \pm}(t, x)\right)$, where $\rho_{ \pm}(p, s)$ and $c_{ \pm}(p, s)$ are the equations of state defined in (8).

We will say that $\left(U_{+}, U_{-}, f\right)$ is a solution of the equations in the fixed domains on the time interval $(0, T)$ with lifting operator $L^{a}$ and initial data $\left(U_{+}^{0}, U_{-}^{0}, f^{0}\right):=$ $\left(\left.U_{+}\right|_{t=0},\left.U_{-}\right|_{t=0},\left.f\right|_{t=0}\right)$ provided that $\rho_{ \pm}(t, x)>0$ and $J^{\psi}(t, x)>0$ in $(0, T) \times$ $\Omega_{ \pm}$, and $p_{ \pm}, u_{ \pm}, s_{ \pm}, f$ satisfy the following system of PDEs:

$$
\begin{gathered}
\frac{1}{\rho c^{2}}\left(\partial_{t}+u^{\psi} \cdot \nabla\right) p+\nabla^{\psi} \cdot u=0 \\
\rho\left(\partial_{t}+u^{\psi} \cdot \nabla\right) u+\nabla^{\psi} p=0 \\
\left(\partial_{t}+u^{\psi} \cdot \nabla\right) s=0
\end{gathered}
$$

in $(0, T) \times \Omega_{ \pm}$

$$
\begin{gathered}
\partial_{t} f=u_{ \pm} \cdot n \\
{[p]=-\sigma \nabla^{\prime} \cdot \hat{n}}
\end{gathered}
$$


on $(0, T) \times \Gamma$, where $\psi$ is a lift of $f$ defined by

$$
\psi:=L^{a} f
$$

Remark 2. Note that for $\psi$ defined by (30), because of the properties of the lifting operator, we have the following on the interface $\Gamma$ :

$$
\begin{array}{r}
J^{\psi}=1 \\
\nabla^{\psi}=\nabla^{\prime}+n \partial_{x_{3}}
\end{array}
$$

on $\Gamma$. Also, if $u$ and $f$ satisfy the Equation (28), then we have the following on the interface $\Gamma$ :

$$
u^{\psi}=\left(u^{\prime}, 0\right)
$$

\subsection{The Initial Data and the Compatibility Conditions}

Definition 12. We define initial data for the system (25)-(30) in the same way as we did for the moving equations in Definition 4 , replacing $\Omega_{ \pm}^{0}$ with $\Omega_{ \pm}$. In addition, we use the obvious notation

$$
\psi^{0}:=L^{a} f^{0}
$$

and we require

$$
J^{\psi^{0}}:=1+\partial_{x_{3}} \psi^{0}>0 \text { for all } x \in \Omega_{ \pm} \text {. }
$$

Definition 13. In the same way as we defined the initial time derivatives in Definition 5, we may define the initial time derivatives $\partial_{0}^{j}$ associated with the equations in the fixed domains, which act on differentiable expressions involving the initial data $\left(U_{+}^{0}, U_{-}^{0}, f^{0}\right)$. Note that we have used the same notation $\partial_{0}^{j}$ as we used for the moving domains in Definition 5 because from now on, with the exception of Section 6, we will be working in the fixed domains.

Definition 14. Let $\left(p_{ \pm}^{0}, u_{ \pm}^{0}, s_{ \pm}^{0}, f^{0}\right)$ be initial data for the system (25)-(30). We say that the initial data $\left(p_{ \pm}^{0}, u_{ \pm}^{0}, s_{ \pm}^{0}, f^{0}\right)$ satisfy the compatibility conditions for the system (25)-(30) up to order $k$, for integer $k \geqq 0$, if the following holds:

$$
\partial_{0}^{j}\left[u^{0} \cdot n^{0}\right]=0
$$

for $0 \leqq j \leqq k$ and

$$
\left[\partial_{0}^{j} p^{0}\right]=-\sigma \partial_{0}^{j}\left(\nabla^{\prime} \cdot \hat{n}^{0}\right)
$$

on $\Gamma$ for $0 \leqq j \leqq k$ 


\subsection{The Energy Functional}

Definition 15. We define the energy $E$ and initial energy $E^{0}$ for the fixed equations exactly as we did for the moving equations as in Definition 7 but with $\Omega_{ \pm}(t)$ replaced by $\Omega_{ \pm}$.

Definition 16. We define the energy $E_{\Delta}:(0, T] \rightarrow \mathbb{R}_{\geq_{0}}$ of the difference of the states $\left(U_{+}^{i}, U_{-}^{i}, f^{i}\right)$ for $i=1,2$ defined on the time interval $(0, T)$ as

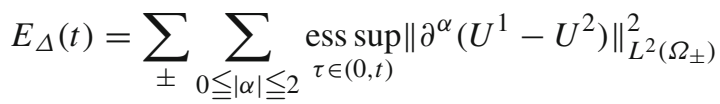

$$
\begin{aligned}
& +\sum_{0 \leqq|\alpha| \leqq 2} \operatorname{ess} \sup \left\|\partial^{\alpha}\left(f^{1}-f^{2}\right)\right\|_{H^{1}(\Gamma)}^{2} \\
& +\sum_{0 \leqq|\alpha| \leqq 1} \operatorname{ess} \sup \left\|\partial^{\alpha}\left(f^{1}-f^{2}\right)\right\|_{H^{2.5}(\Gamma)}^{2} \text {. }
\end{aligned}
$$

Note that this is effectively the energy $E(t)$ reduced by one order of regularity evaluated at the difference of two solutions.

Similarly, we define the initial energy $E_{\Delta}^{0}$ of the difference of two sets of initial data $\left(U_{+}^{0, i}, U_{-}^{0, i}, f^{0, i}\right)$ for $i=1,2$ as

$$
\begin{aligned}
E_{\Delta}^{0}= & \sum_{ \pm} \sum_{0 \leqq|\beta|+j \leqq 2}\left\|\nabla^{\beta}\left(\partial_{0}^{j, 1} U^{0,1}-\partial_{0}^{j, 2} U^{0,2}\right)\right\|_{L^{2}\left(\Omega_{ \pm}\right)}^{2} \\
& +\sum_{0 \leqq|\beta|+j \leqq 2}\left\|\nabla^{\beta}\left(\partial_{0}^{j, 1} f^{0,1}-\partial_{0}^{j, 2} f^{0,2}\right)\right\|_{H^{1}(\Gamma)}^{2} \\
& +\sum_{0 \leqq|\beta|+j \leqq 1}\left\|\nabla^{\beta}\left(\partial_{0}^{j, 1} f^{0,1}-\partial_{0}^{j, 2} f^{0,2}\right)\right\|_{H^{2.5}(\Gamma)}^{2}
\end{aligned}
$$

where $\partial_{0}^{j, i}$ denotes the initial time derivatives of order $j$ as defined in Definition 13 acting on the initial data $\left(U_{+}^{0, i}, U_{-}^{0, i}, f^{0, i}\right)$ for $i=1,2$.

\subsection{Statement of the Theorem in the Fixed Domains}

Theorem 2. Let $\left(U_{+}^{0}, U_{-}^{0}, f^{0}\right)$ be initial data as described in Definition 12 with energy $E^{0}<\infty$. Assume that this initial data satisfies the compatibility conditions (31)-(32) up to order 2, and note that these conditions make sense up to order 2 since we have $E^{0}<\infty$. Assume also that the initial data satisfies

$$
\begin{aligned}
\inf _{x \in \Omega_{ \pm}} \rho_{ \pm}^{0}(x) & =: \delta^{0}>0 \\
\inf _{x \in \mathbb{R}^{3}} J^{\psi^{0}} & =: \kappa^{0}>0 .
\end{aligned}
$$


Then there exists a time $T^{0}>0$ and a solution $\left(U_{+}, U_{-}, f\right)$ of equations in the fixed domains on the time interval $\left(0, T^{0}\right)$ with lifting operator $L^{a}$, as in Definition 11. Moreover,

$$
E\left(T^{0}\right) \leqq C^{0}, \quad \rho_{ \pm} \geqq \frac{\delta^{0}}{2} \quad \text { in } \quad\left(0, T^{0}\right) \times \Omega_{ \pm}, \quad J^{\psi} \geqq \frac{1}{2} \kappa^{0} \quad \text { in } \quad\left(0, T^{0}\right) \times \mathbb{R}^{3}
$$

The time $T^{0}>0$ is bounded below as follows:

$$
T^{0} \geqq T\left(E^{0}, \frac{1}{\delta^{0}}, \frac{1}{\kappa^{0}}\right)>0
$$

where $T(\cdot)$ is a smooth decreasing function. Similarly, the constant $C^{0}>0$ is bounded above as follows:

$$
C^{0} \leqq C\left(E^{0}, \frac{1}{\delta^{0}}, \frac{1}{\kappa^{0}}\right)<\infty,
$$

where $C(\cdot)$ is a smooth increasing function. Note that these bounds are independent of the constant a in the lifting operator $L^{a}$.

In addition, the solution $\left(U_{+}, U_{-}, f\right)$ is unique on the time interval $\left(0, T^{0}\right)$ in the sense that it is the only solution of the equations given in Definition 11 on the time interval $\left(0, T^{0}\right)$ with initial data $\left(U_{+}^{0}, U_{-}^{0}, f^{0}\right)$ satisfying the following properties:

$$
E\left(T^{0}\right)<\infty, \quad \inf _{t \in\left(0, T^{0}\right)} \inf _{x \in \Omega_{ \pm}} \rho_{ \pm}>0, \inf _{t \in\left(0, T^{0}\right)} \inf _{x \in \mathbb{R}^{3}} J^{\psi}>0 .
$$

In fact, the following stability statement is satisfied. Given two sets of initial data $\left(U_{+}^{0, i}, U_{-}^{0, i}, f^{0, i}\right)$ for $i=1,2$ satisfying the hypotheses above, let $T^{0,1}$ and $T^{0,2}$ be the associated existence times as given above and set $T^{0}=\min \left\{T^{0,1}, T^{0,2}\right\}$. Let $\left(U_{+}^{1}, U_{-}^{1}, f^{1}\right)$ and $\left(U_{+}^{2}, U_{-}^{2}, f^{2}\right)$ be two solutions of the equations in the fixed domains as defined in Definition 11 on the time interval $\left(0, T^{0}\right)$ with initial data $\left(U_{+}^{0, i}, U_{-}^{0, i}, f^{0, i}\right)$ respectively, with the properties

$$
E^{i}\left(T^{0}\right)=: C^{i}<\infty, \inf _{t \in\left(0, T^{0}\right)} \inf _{x \in \Omega_{ \pm}} \rho_{ \pm}^{i}=: \delta^{i}>0, \inf _{t \in\left(0, T^{0}\right)} \inf _{x \in \mathbb{R}^{3}} J^{\psi^{i}}=: \kappa^{i}>0
$$

for $i=1,2$, where the superscript ${ }^{i}$ for $i=1,2$ is used to denote quantities associated with the solutions $\left(U_{+}^{i}, U_{-}^{i}, f^{i}\right)$. Assume also that $U^{0,1}-U^{0,2} \in L^{2}\left(\Omega_{ \pm}\right)$ and $f^{0,1}-f^{0,2} \in L^{2}(\Gamma)$ so that

$$
E_{\Delta}^{0}<\infty
$$

where the initial difference energy $E_{\Delta}^{0}$ is defined in Definition 16. Then

$$
E_{\Delta}(t) \leqq C^{1,2} E_{\Delta}^{0} \exp \left(C^{1,2} t\right)
$$

for $t \in\left(0, T^{0}\right)$ where $E_{\Delta}(t)$ is defined in Definition 16 and the constant $C^{1,2}$ is bounded above as follows:

$$
C^{1,2} \leqq F\left(C^{1}, C^{2}, \frac{1}{\delta^{1}}, \frac{1}{\delta^{2}}, \frac{1}{\kappa^{1}}, \frac{1}{\kappa^{2}}\right),
$$

for some smooth increasing function $F(\cdot)$.

Proof. See Sections 7-15. 


\section{Fixing the Domains}

In this section we show how the equations and theorem in the fixed domains relate to the equations and theorem in the original moving domains.

\subsection{The Change of Coordinates}

Lemma 1. Let $f \in C^{1}\left((0, T) \times \mathbb{R}^{2}\right)$ and let $a \geqq 0$ be a constant. Assume that

$$
\inf _{t \in(0, T)} \inf _{x \in \mathbb{R}^{3}} J^{\psi} \geqq \kappa>0
$$

where $\psi=L^{a} f, J^{\psi}:=1+\partial_{x_{3}} \psi$ and the lifting operator $L^{a}$ is defined in Definition 9. Define $\theta_{ \pm} \in C^{1}\left((0, T) \times \Omega_{ \pm}\right)$by

$$
\theta_{ \pm}(t, x)=\left(t, x^{\prime}, x_{3}+\psi(t, x)\right)
$$

Then $\theta_{ \pm}:(0, T) \times \Omega_{ \pm} \rightarrow \Omega_{ \pm}^{T}$ are diffeomorphisms, where the domains $\Omega_{ \pm}^{T}$ are defined as in Definition 3 in terms of $f$.

Moreover, if $w \in C^{1}\left(\Omega_{ \pm}^{T}\right)$ and we set $\tilde{w}=w \circ \theta$, then

$$
(\partial w) \circ \theta=\left(\partial-\frac{1}{J \psi} \partial \psi \partial_{x_{3}}\right) \tilde{w}=: \partial^{\psi} \tilde{w} .
$$

In particular, note that if in addition $v \in C^{0}\left(\Omega_{ \pm}^{T}\right)$ is a vector-valued function and $\tilde{v}:=v \circ \theta$, then

$$
\left(\left(\partial_{t}+v \cdot \nabla\right) w\right) \circ \theta=\left(\partial_{t}+\tilde{v}^{\psi} \cdot \nabla\right) \tilde{w}
$$

where

$$
\tilde{v}^{\psi}:=\left(\tilde{v}^{\prime}, \frac{1}{J \psi}\left(\tilde{v}_{3}-\tilde{v}^{\prime} \cdot \nabla^{\prime} \psi-\partial_{t} \psi\right)\right) .
$$

Proof. Note that $J^{\psi}:=1+\partial_{x_{3}} \psi \geqq \kappa>0$ implies $x_{3} \mapsto x_{3}+\psi(t, x)$ is a strictly increasing function of $x_{3}$ for fixed $\left(t, x^{\prime}\right)$. Hence, since $\left(t, x^{\prime}\right) \mapsto\left(t, x^{\prime}\right)$, we have that $\theta_{ \pm}$biject with their images. Now, since $\left.\psi\right|_{x_{3}=0}=f$, we have that $\left.\theta_{ \pm}\right|_{x_{3}=0}:(0, T) \times \Gamma \rightarrow \Gamma^{T}$ bijectively, and since $x_{3} \mapsto x_{3}+\psi(t, x)$ is strictly increasing, $\theta_{ \pm}:(0, T) \times \Omega_{ \pm} \rightarrow \Omega_{ \pm}^{T}$ bijectively. The rest is a simple application of the chain rule.

Proposition 1. Let $T>0, a \geqq 0$ and let $f \in C_{b}^{1}\left(\left(0, T^{\prime}\right) \times \mathbb{R}^{2}\right)$ with $\nabla^{\prime} f \in$ $C_{b}^{1}\left(\left(0, T^{\prime}\right) \times \mathbb{R}^{2}\right)$ for all $T^{\prime} \in(0, T)$. Assume that

$$
\inf _{t \in(0, T)} \inf _{x \in \mathbb{R}^{3}} J^{\psi} \geqq \kappa>0
$$

where $\psi=L^{a} f$. Let $\theta_{ \pm}:(0, T) \times \Omega_{ \pm} \rightarrow \Omega_{ \pm}^{T}$ be defined in terms of $f$ as in Lemma 1. Let $\tilde{U}_{ \pm} \in C_{b}^{1}\left(\left(0, T^{\prime}\right) \times \Omega_{ \pm}\right)$for all $T^{\prime} \in(0, T)$ and let

$$
U_{ \pm}=\tilde{U}_{ \pm} \circ \theta^{-1} .
$$


Then $\left(\tilde{U}_{+}, \tilde{U}_{-}, f\right)$ is a solution of the equations in the fixed domains $\Omega_{ \pm}$with lifting operator $L^{a}$ on the time interval $(0, T)$, as in Definition 11 , if and only if $\left(U_{+}, U_{-}, f\right)$ is a solution of the equations in the moving domains $\Omega_{ \pm}^{T}$ on the time interval $(0, T)$, as in Definition 3.

Proof. Firstly, note that $\rho_{ \pm}>0$ if and only if $\tilde{\rho}_{ \pm}>0$ since they have the same image.

Using the coordinate change relations (35)-(36), we immediately see $\left(\tilde{U}_{+}, \tilde{U}_{-}\right.$, $f)$ satisfies the Equations (26)-(27) in $(0, T) \times \Omega_{ \pm}$if and only if $\left(U_{+}, U_{-}, f\right)$ satisfies the Equations (10)-(11) in $\Omega_{ \pm}^{T}$. We note that $\left.U_{ \pm}\right|_{\Gamma(t)}=\left.\tilde{U}_{ \pm}\right|_{\Gamma}$, which immediately implies that $\left(\tilde{U}_{+}, \tilde{U}_{-}, f\right)$ satisfies the Equations (28)-(29) on $(0, T) \times$ $\Gamma$ if and only if $\left(U_{+}, U_{-}, f\right)$ satisfies the Equations (12)-(13) on $\Gamma^{T}$.

This completes the proof.

Proposition 2. Let $\left(U_{+}^{0}, U_{-}^{0}, f^{0}\right)$ be initial data for the main theorem, Theorem 1.

Let $\psi^{0}:=L^{a} f^{0}$ as defined by (9) and assume $J^{\psi^{0}}>0$. Define $\tilde{U}_{ \pm}^{0}:=$ $U_{ \pm}^{0} \circ \theta_{ \pm}^{0}$ where $\theta_{ \pm}^{0}: \Omega_{ \pm} \rightarrow \Omega_{ \pm}^{0}$ are defined by $x \mapsto\left(x^{\prime}, x_{3}+\psi^{0}(x)\right)$ and are diffeomorphisms as shown in Proposition 1.

Then $\left(\tilde{U}_{+}^{0}, \tilde{U}_{-}^{0}, f^{0}\right)$ are initial data for the theorem in the fixed domains, Theorem 2.

Moreover, let us write $\partial_{0}^{j}$ for the initial time derivatives associated with the equations in the moving domains as defined in Definition 5 and $\tilde{\partial}_{0}^{j}$ for the initial time derivatives associated with the equations in the fixed domains as defined in Definition 13.

Let $H\left(p_{ \pm}^{0}, u_{ \pm}^{0}, s_{ \pm}^{0}, f^{0}\right)$ be a differentiable expression involving the initial data $\left(U_{+}^{0}, U_{-}^{0}, f^{0}\right)$ and suppose we can write

$$
H\left(p_{ \pm}^{0}, u_{ \pm}^{0}, s_{ \pm}^{0}, f^{0}\right) \circ \theta_{ \pm}^{0}=\tilde{H}\left(\tilde{p}_{ \pm}^{0}, \tilde{u}_{ \pm}^{0}, \tilde{s}_{ \pm}^{0}, f^{0}\right)
$$

where $\tilde{H}\left(\tilde{p}_{ \pm}^{0}, \tilde{u}_{ \pm}^{0}, \tilde{s}_{ \pm}^{0}, f^{0}\right)$ is a differentiable expression involving the initial data in the fixed domains $\left(\tilde{U}_{+}^{0}, \tilde{U}_{-}^{0}, f^{0}\right)$. Then for $j \geqq 0$, we have

$$
\tilde{\partial}_{0}^{j}\left(\tilde{H}\left(\tilde{p}_{ \pm}^{0}, \tilde{u}_{ \pm}^{0}, \tilde{s}_{ \pm}^{0}, f^{0}\right)\right)=\partial_{0}^{j}\left(H\left(p_{ \pm}^{0}, u_{ \pm}^{0}, s_{ \pm}^{0}, f^{0}\right) \circ \theta_{ \pm}^{0}\right) .
$$

Proof. This is straightforward to check using the definition of the initial time derivatives.

\subsection{Reduction to the Case of Fixed Domains}

Proposition 3. Theorem 1 in the moving domains follows from Theorem 2 in the fixed domains.

Proof. Assume that Theorem 2 holds. We aim to show that Theorem 1 holds.

Let $\left(U_{+}^{0}, U_{-}^{0}, f^{0}\right)$ be initial data for the main theorem, Theorem 1 , which satisfy the hypotheses of this theorem.

Let $\psi^{0}:=L^{a} f^{0}$ as defined by (9), where we set

$$
a=\left\|f^{0}\right\|_{L^{\infty}(\Gamma)} .
$$


Note from the definition of $L^{a}$ that this automatically implies

$$
\left\|\partial_{x_{3}} \psi^{0}\right\|_{L^{\infty}\left(\mathbb{R}^{3}\right)} \leqq \frac{1}{3} .
$$

so

$$
J^{\psi^{0}}:=1+\partial_{x_{3}} \psi^{0} \geqq \kappa^{0}>0 \text { in } \mathbb{R}^{3},
$$

where in this case we can take $\kappa^{0}=\frac{2}{3}$. Define $\tilde{U}_{ \pm}^{0}:=U_{ \pm}^{0} \circ \theta_{ \pm}^{0}$ where $\theta_{ \pm}^{0}: \Omega_{ \pm} \rightarrow$ $\Omega_{ \pm}^{0}$ are defined by $x \mapsto\left(x^{\prime}, x_{3}+\psi^{0}(x)\right)$ and are diffeomorphisms as shown in Lemma 1.

It is easy to check using Proposition 2 that $\left(\tilde{U}_{+}^{0}, \tilde{U}_{-}^{0}, f^{0}\right)$ are initial data for the theorem on the fixed domains, Theorem 2, which satisfy the hypotheses of this theorem. Note that we obtain the energy relation

$$
\tilde{E}^{0} \leqq \tilde{F}\left(E^{0}\right)<\infty
$$

where $\tilde{F}$ is a smooth increasing function by using the Sobolev embedding theorem, the chain rule, and the fact that $\theta_{ \pm}^{0}$ are Lipschitz diffeomorphisms. Here, $E^{0}$ denotes the energy of $\left(U_{+}^{0}, U_{-}^{0}, f^{0}\right)$ as defined in Definition 7 and $\tilde{E}^{0}$ denotes the energy of $\left(\tilde{U}_{+}^{0}, \tilde{U}_{-}^{0}, f^{0}\right)$ as defined in Definition 15. Clearly, we have inf ${ }_{x \in \Omega_{ \pm}} \tilde{\rho}_{ \pm}^{0}=\delta^{0}>0$ since $\tilde{\rho}_{ \pm}^{0}$ and $\rho_{ \pm}^{0}$ have the same image. Now we let $T^{0}>0$ be given by Theorem 2 depending on this initial data.

It is now straightforward to show that the existence part of Theorem 1 follows by applying Theorem 2 to obtain a solution $\left(\tilde{U}_{+}, \tilde{U}_{-}, f\right)$ on a time interval $\left(0, T^{0}\right)$ of the system (25)-(30) with initial data $\left(\tilde{U}_{+}^{0}, \tilde{U}_{-}^{0}, f^{0}\right)$ and then showing that composition of $\tilde{U}_{ \pm}$with $\theta^{-1}$, where $\theta^{-1}$ is constructed from $f$ as in Lemma 1 , is the solution to the equations on the moving domains that we seek, for which we use Proposition 1.

To prove the existence part, we start with two solutions $\left(U_{+}^{i}, U_{-}^{i}, f^{i}\right)$ for $i=$ 1,2 on the moving domains satisfying the stated properties required for uniqueness, defined on the time interval $\left(0, T^{0}\right)$ where $T^{0}$ is as stated above, and depends only on the initial data. We then compose with $\theta^{i}$ (constructed from $f^{i}$ ) to obtain two solutions to the equations on the fixed domains, then we apply the uniqueness result for the fixed domains to conclude they are equal.

The sections which follow will be devoted to proving Theorem 2 in the fixed domains, from which we have shown that Theorem 1 follows.

\section{Smoothing the Initial Data}

Here we show that it is sufficient to prove Theorem 2 for smooth initial data.

\subsection{Constructing Smooth Compatible Initial Data}

Lemma 2. Let $l \geqq 0$ be an integer and $d \geqq 2$ be an integer. Let $h^{k} \in$ $H^{l-k+0.5}\left(\mathbb{R}^{d-1}\right)$ for $0 \leqq k \leqq l$. Then there exists $g \in H^{l+1}\left(\mathbb{R}^{d}\right)$ such that 


$$
\operatorname{Tr}\left(\partial_{x_{d}}^{k} g\right)=h^{k} \text { for } 0 \leqq k \leqq l
$$

and

$$
\|g\|_{H^{l+1}\left(\mathbb{R}^{d}\right)} \leqq C \sum_{k=0}^{l}\left\|h^{k}\right\|_{H^{l-k+0.5}\left(\mathbb{R}^{d-1}\right)}
$$

Moreover, the map $\mathcal{L}:\left(h^{0}, \ldots, h^{l}\right) \mapsto g$ is linear.

Proof. This is a fairly standard result. We sketch the proof as follows.

Write $x=\left(x^{\prime}, x_{d}\right) \in \mathbb{R}^{d}$ with $x^{\prime} \in \mathbb{R}^{d-1}$. Let $\chi \in C_{c}^{\infty}(\mathbb{R})$ be a smooth positive cut-off function with $\chi(s)=1$ for $|s| \leqq 1$. We define $g: \mathbb{R}^{d} \rightarrow \mathbb{R}$ by

$$
g(x)=\sum_{k=0}^{l} \frac{1}{k !} x_{d}^{k} \chi\left(x_{d}\left\langle D^{\prime}\right\rangle\right) h^{k}\left(x^{\prime}\right):=\sum_{k=0}^{l} \mathcal{F}^{-1}\left(\frac{1}{k !} x_{d}^{k} \chi\left(x_{d}\left\langle\xi^{\prime}\right\rangle\right) \mathcal{F}\left(h^{k}\right)\left(\xi^{\prime}\right)\right)
$$

where $\mathcal{F}$ denotes the Fourier transform. One can easily check that the map $\mathcal{L}$ : $\left(h^{0}, \ldots, h^{l}\right) \mapsto g$ has the required properties.

Proposition 4. Let $\left(U_{+}^{0}, U_{-}^{0}, f^{0}\right)$ be initial data which satisfy the hypotheses of Theorem 2, so in particular satisfy the compatibility conditions (31)-(32) up to order 2. Then for $\varepsilon>0$ there are functions $U_{\varepsilon \pm}^{0}=\left(p_{\varepsilon \pm}^{0}, u_{\varepsilon \pm}^{0}, s_{\varepsilon \pm}^{0}\right) \in C^{\infty}\left(\Omega_{ \pm}\right), f_{\varepsilon}^{0} \in$ $C^{\infty}\left(\mathbb{R}^{2}\right)$ which form a set of initial data for the system (25)-(30).

We also have

$$
\partial_{0}^{\varepsilon, j} \nabla^{\beta} U_{\varepsilon \pm}^{0} \in L^{2}\left(\Omega_{ \pm}\right), \quad \partial_{0}^{\varepsilon, j} \nabla^{\beta} f_{\varepsilon}^{0} \in L^{2}(\Gamma)
$$

for all $j+|\beta| \geqq 1$, where we write $\partial_{0}^{j}$ for the initial time derivatives associated with the initial data $\left(U_{+}^{0}, U_{-}^{0}, f^{0}\right)$ and $\partial_{0}^{\varepsilon, j}$ for the initial time derivatives associated with the initial data $\left(U_{\varepsilon+}^{0}, U_{\varepsilon-}^{0}, f_{\varepsilon}^{0}\right)$.

Additionally, this set of initial data satisfies the compatibility conditions given in Definition 14 up to order 2. Also, it converges to the original initial data as $\varepsilon \rightarrow 0$ in the following sense:

$$
\begin{aligned}
&\left\|U_{\varepsilon}^{0}-U^{0}\right\|_{L^{\infty}\left(\Omega_{ \pm}\right)} \rightarrow 0 \text { as } \varepsilon \rightarrow 0 \\
&\left\|\partial_{0}^{\varepsilon, j} \nabla^{\beta} U_{\varepsilon}^{0}-\partial_{0}^{j} \nabla^{\beta} U^{0}\right\|_{L^{2}\left(\Omega_{ \pm}\right)} \rightarrow 0 \text { as } \varepsilon \rightarrow 0 \text { for } 0 \leqq|\beta|+j \leqq 3 \\
&\left\|f_{\varepsilon}^{0}-f^{0}\right\|_{L^{\infty}\left(\mathbb{R}^{2}\right)} \rightarrow 0 \text { as } \varepsilon \rightarrow 0 \\
&\left\|\partial_{0}^{\varepsilon, j} \nabla^{\beta} f_{\varepsilon}^{0}-\partial_{0}^{j} \nabla^{\beta} f^{0}\right\|_{H^{1}\left(\mathbb{R}^{2}\right)} \rightarrow 0 \text { as } \varepsilon \rightarrow 0 \text { for } 0 \leqq|\beta|+j \leqq 3 \\
&\left\|\partial_{0}^{\varepsilon, j} \nabla^{\beta} f_{\varepsilon}^{0}-\partial_{0}^{j} \nabla^{\beta} f^{0}\right\|_{H^{2.5}\left(\mathbb{R}^{2}\right)} \rightarrow 0 \text { as } \varepsilon \rightarrow 0 \text { for } 0 \leqq|\beta|+j \leqq 2 .
\end{aligned}
$$

In particular,

$$
E_{\varepsilon}^{0} \rightarrow E^{0} \text { as } \quad \varepsilon \rightarrow 0 .
$$


Using the obvious notation

$$
\rho_{\varepsilon \pm}^{0}(x):=\rho_{ \pm}\left(p_{\varepsilon \pm}^{0}(x), s_{\varepsilon \pm}^{0}(x)\right), \quad \psi_{\varepsilon}^{0}:=L^{a} f_{\varepsilon}^{0},
$$

we have

$$
\inf _{x \in \Omega_{ \pm}} \rho_{\varepsilon \pm}^{0}=: \delta_{\varepsilon}^{0} \geqq \frac{\delta^{0}}{2} \quad \text { in } \quad \Omega_{ \pm}, \quad \inf _{x \in \Omega_{ \pm}} J^{\psi_{\varepsilon}^{0}}=: \kappa_{\varepsilon}^{0} \geqq \frac{1}{2} \kappa^{0}
$$

for all $\varepsilon>0$, where $\delta^{0}>0$ and $\kappa^{0} \in(0,1)$ are defined as

$$
\inf _{x \in \Omega_{ \pm}} \rho_{ \pm}^{0}=: \delta^{0}, \quad \inf _{x \in \Omega_{ \pm}} J^{\psi^{0}}=: \kappa^{0}
$$

and we have

$$
\delta_{\varepsilon}^{0} \rightarrow \delta^{0} \quad \text { as } \quad \varepsilon \rightarrow 0, \quad \kappa_{\varepsilon}^{0} \rightarrow \kappa^{0} \quad \text { as } \quad \varepsilon \rightarrow 0 .
$$

Proof. We give a sketch of the proof. We mollify $\left(U_{+}^{0}, U_{-}^{0}, f^{0}\right)$ by convolution with the standard mollifier (applying Sobolev extension first as necessary) to obtain $\tilde{p}_{\varepsilon \pm}^{0}, \tilde{u}_{\varepsilon \pm}^{0}, \tilde{s}_{\varepsilon \pm}^{0}, \tilde{f}_{\varepsilon}^{0}$. We then set the following:

$$
s_{\varepsilon \pm}^{0}=\tilde{s}_{\varepsilon \pm}^{0}, \quad f_{\varepsilon}^{0}=\tilde{f}_{\varepsilon}^{0}, \quad\left(u_{\varepsilon+}^{0}\right)^{\prime}=\left(\tilde{u}_{\varepsilon+}^{0}\right)^{\prime}, \quad\left(u_{\varepsilon-}^{0}\right)^{\prime}=\left(\tilde{u}_{\varepsilon-}^{0}\right)^{\prime} .
$$

We then add correction terms to $\tilde{p}_{\varepsilon+}^{0}$ and $\left(\tilde{u}_{\varepsilon}^{0}\right)_{3+}$ to produce $p_{\varepsilon+}^{0}$ and $\left(u_{\varepsilon}^{0}\right)_{3+}$ so that $\partial_{0}^{\varepsilon, j}\left(u_{\varepsilon+}^{0} \cdot n_{\varepsilon}^{0}\right)=\partial_{0}^{j}\left(u^{0} \cdot n^{0}\right) * \eta_{\varepsilon}$ for $0 \leqq j \leqq 2$, where here $\eta_{\varepsilon}$ is the mollifier in two space dimensions. This is done by writing the initial time derivatives on the boundary in terms of normal derivatives (done using the definition of the initial time derivatives plus an inductive argument), which allows us to specify the normal derivatives of the correction term needed on $\Gamma$ in terms of $\tilde{p}_{\varepsilon+}^{0}, \tilde{u}_{\varepsilon+}^{0}, \tilde{s}_{\varepsilon+}^{0}, \tilde{f}_{\varepsilon}^{0}$ and we use Lemma 2 to construct a correction term in the interior with the desired normal derivatives on the boundary.

Similarly, we add correction terms to $\tilde{p}_{\varepsilon-}^{0}$ and $\left(\tilde{u}_{\varepsilon}^{0}\right)_{3-}$ to produce $p_{\varepsilon-}^{0}$ and $\left(u_{\varepsilon}^{0}\right)_{3-}$ so that the compatibility conditions (31)-(32) up to order 2 are satisfied up to order 2 by the smoothed initial data.

Note that since we have used Lemma 2 to construct our correction terms with the same regularity as $U_{ \pm}^{0}$ uniformly in $\varepsilon$, the correction terms disappear as $\varepsilon \rightarrow 0$ by virtue of the fact that the initial data $\left(U_{+}^{0}, U_{-}^{0}, f^{0}\right)$ satisfies the compatibility conditions up to order 2 , and the claimed convergences hold.

Proposition 5. Let $\left(U_{+}^{0}, U_{-}^{0}, f^{0}\right)$ be initial data which satisfy the hypotheses of Theorem 2, so in particular satisfy the compatibility conditions up to order 2 . Then there exists a sequence of initial data $\left(U_{m+}^{0}, U_{m-}^{0}, f_{m}^{0}\right), m \geqq 1$, which satisfies all the conclusions of Proposition 4 (replacing $\varepsilon \rightarrow 0$ with $m \rightarrow \infty$ ) and in addition each element of the sequence satisfies the compatibility condition (31) up to order 3.

Proof. We smooth the initial data as in Proposition 4 then add a correction term $\frac{1}{3 !} x_{3}^{3} \chi\left(\frac{x_{3}}{\eta}\right) q_{\varepsilon-}^{0}$ to $p_{\varepsilon}^{0}$ to produce $p_{\varepsilon, \eta-}^{0}$ for $\eta>0$ where $q_{\varepsilon-}^{0}$ is chosen such that the initial data with this corrected pressure satisfies the compatibility condition (31) up 
to order 3 . We then extract a subsequence $\left(\varepsilon_{m}, \eta_{m}\right)$ where we first choose $\varepsilon_{m}$ then $\eta_{m}$ to ensure convergence.

\subsection{Reduction to the Case of Smooth Initial Data}

Proposition 6. Suppose we can prove the existence part of Theorem 2 under the additional assumption that the initial data $\left(U_{+}^{0}, U_{-}^{0}, f^{0}\right)$ are smooth, ie $\nabla^{\alpha} U_{ \pm}^{0} \in$ $L^{2}\left(\Omega_{ \pm}\right)$and $\nabla^{\alpha^{\prime}} f^{0} \in L^{2}\left(\mathbb{R}^{2}\right)$ for all $|\alpha| \geqq 1$, and that the compatibility condition (31) holds up to order 3. Then Theorem 2 follows (without the smoothness assumption).

Proof. This is a straightforward, if technical, consequence of Proposition 5 together with the energy estimate and bounds on the constants $C^{0}$ and $T^{0}$ in terms of the size of the initial data given in the statement of Theorem 2 and weak-* compactness, the Sobolev embedding theorem and the Sobolev compact embedding theorem on bounded domains.

\section{The $\mu$-Approximate Equations}

Here we define a sort of viscous regularisation of the Equations (25)-(30) on the fixed domains. For brevity we do not redefine quantities whose definition is obvious given notation we have introduced previously. Also, we will not write explicitly the dependence on $\mu$ of a solution to the $\mu$-approximate equations else the notation would be too messy.

\subsection{Definition of the $\mu$-Approximate Equations}

Definition 17. For $\mu>0$, given the density functions $\rho_{ \pm}(t) \in C_{b}^{1}\left(\Omega_{ \pm}\right)$for each $t$ we define the smoothed density $\rho_{\mu \pm}$ as

$$
\rho_{\mu \pm}=\left.\left(\operatorname{Ext}_{\Omega_{ \pm}} \rho_{ \pm}\right) * \eta_{\mu}\right|_{\Omega_{ \pm}}
$$

where the Sobolev extension operators $\operatorname{Ext}_{\Omega_{ \pm}}$are defined in Definition 2 and the standard mollifier $\eta_{\mu}$ is defined in Definition 1 . Note that we mollify only in space, not in time. We also apply this definition to the initial data $\rho_{ \pm}^{0}$.

Note that by properties of mollification and Sobolev extension,

$$
\left\|\rho_{\mu}-\rho\right\|_{L^{\infty}\left(\Omega_{ \pm}\right)} \leqq \mu C\|\nabla \rho\|_{L^{\infty}\left(\Omega_{ \pm}\right)}
$$

hence

$$
\left\|\rho_{\mu}-\rho\right\|_{L^{\infty}\left(\Omega_{ \pm}\right)} \rightarrow 0 \text { as } \mu \rightarrow 0
$$

provided $\|\nabla \rho\|_{L^{\infty}\left(\Omega_{ \pm}\right)}$is bounded. 
Definition 18. Let $\mu \in(0,1]$.

Fix $T \in(0, \infty)$. Also fix $\left(g_{1}\right)_{ \pm} \in C_{b}\left((0, T) \times \Omega_{ \pm} ; \mathbb{R}^{3}\right), g_{2} \in C_{b}((0, T) \times \Gamma)$ and write $g=\left(g_{1}, g_{2}\right)$ which we will call the right hand side of the equations.

Let $f \in C_{b}^{1}\left(\left(0, T^{\prime}\right) \times \mathbb{R}^{2}\right)$ with $\nabla^{\prime} f \in C_{b}^{1}\left(\left(0, T^{\prime}\right) \times \mathbb{R}^{2}\right)$ for all $T^{\prime} \in(0, T)$. Let $U_{ \pm}=\left(p_{ \pm}, u_{ \pm}, s_{ \pm}\right) \in C_{b}^{1}\left(\left(0, T^{\prime}\right) \times \Omega_{ \pm}\right)$for all $T^{\prime} \in(0, T)$. In addition, assume $u_{ \pm} \in C_{b}^{2}\left(\Omega_{ \pm}\right),\left.u_{ \pm}\right|_{\Gamma} \cdot n \in C_{b}^{2}\left(\mathbb{R}^{2}\right)$ for each $t \in(0, T)$.

We will say that $\left(U_{+}, U_{-}, f\right)$ is a solution of the $\mu$-approximate equations on the time interval $(0, T)$ with lifting operator $L^{a}$ and right hand side $g$, with initial data

$$
\left(U_{+}^{0}, U_{-}^{0}, f^{0}\right):=\left(\left.U_{+}\right|_{t=0},\left.U_{-}\right|_{t=0},\left.f\right|_{t=0}\right)
$$

if $\rho(t, x)>0, \rho_{\mu}(t, x)>0$ and $J^{\psi}(t, x)>0$ in $(0, T) \times \Omega_{ \pm}$, and $p_{ \pm}, u_{ \pm}, s_{ \pm}, f$ satisfy the following system of PDEs:

$$
\begin{aligned}
\frac{1}{\rho c^{2}}\left(\partial_{t}+u^{\psi} \cdot \nabla\right) p+\nabla^{\psi} \cdot u & =0 \\
\rho_{\mu}\left(\partial_{t}+u^{\psi} \cdot \nabla\right) u+\nabla^{\psi} p & =\mu \nabla^{\psi}\left(\rho c^{2} \nabla^{\psi} \cdot u\right)+g_{1} \\
\left(\partial_{t}+u^{\psi} \cdot \nabla\right) s & =0
\end{aligned}
$$

in $(0, T) \times \Omega_{ \pm}$,

$$
\begin{aligned}
\partial_{t} f & =u_{ \pm} \cdot n \\
{\left[p-\mu \rho c^{2} \nabla^{\psi} \cdot u\right] } & =-\sigma \nabla^{\prime} \cdot \hat{n}+\mu\left(\Delta^{\prime}-1\right)(u \cdot n)+g_{2}
\end{aligned}
$$

on $(0, T) \times \Gamma$,

$$
\psi:=L^{a} f
$$

\subsection{Initial Data and the Compatibility Conditions}

Definition 19. In the same way as we defined the initial time derivatives in Definition 5, we may define the initial time derivatives $\partial_{0}^{\mu, g, j}$ associated with the $\mu$-approximate equations with lifting operator $L^{a}$ and right hand side $g$, which act on differentiable expressions involving the initial data $\left(U_{+}^{0}, U_{-}^{0}, f^{0}\right)$.

Definition 20. Let $\left(U_{+}^{0}, U_{-}^{0}, f^{0}\right)$ be initial data for the $\mu$-approximate equations with lifting operator $L^{a}$ and right hand side $g$. We say that $\left(U_{+}^{0}, U_{-}^{0}, f^{0}\right)$ satisfies the normal velocity jump compatibility condition up to order $k$ if

$$
\partial_{0}^{\mu, g, j}\left[u^{0} \cdot n^{0}\right]=0
$$

for $0 \leqq j \leqq k$. We say that $\left(U_{+}^{0}, U_{-}^{0}, f^{0}\right)$ satisfies the pressure interface compatibility condition up to order $k$ if

$$
\begin{aligned}
\partial_{0}^{\mu, g, j}\left[p^{0}-\mu \rho^{0}\left(c^{0}\right)^{2} \nabla^{\psi^{0}} \cdot u^{0}\right]= & \partial_{0}^{\mu, g, j}\left(-\sigma\left(\nabla^{\prime} \cdot \hat{n}^{0}\right)+\mu\left(\Delta^{\prime}-1\right)\left(\left.u^{0}\right|_{\Gamma} \cdot n^{0}\right)\right) \\
& +\left.\partial_{t}^{j} g_{2}(t)\right|_{t=0}
\end{aligned}
$$

for $0 \leqq j \leqq k$. 


\subsection{Energy for the $\mu$-Approximate Equations}

Here, we introduce two separate energies, $E_{\mu}$ and $\mathcal{E}_{\mu}$, associated with the approximate equations. We will call the energy $E_{\mu}$ the $\mu$-independent energy since it will be used in a $\mu$-independent energy estimate and should be thought of as being like the energy $E$ for the equations in the fixed domains as defined in Definition 15 but with some extra terms multiplied by powers of $\mu$ added which correspond to the addition of the terms involving $\mu$ to the equations in the fixed domains, allowing us to close the energy estimate for the $\mu$-approximate equations. We will call the energy $\mathcal{E}_{\mu}$ the higher order $\mu$-approximate energy and we will use this for solutions of the $\mu$-approximate equations with $\mu$ fixed. The advantage of introducing this energy is that we may perform the $\mu$-independent energy estimate on solutions of the $\mu$-approximate equations with $\mathcal{E}_{\mu}(T)<\infty$ without having to worry about whether the solution has enough derivatives for us to differentiate the equations.

Definition 21. Let $f \in L_{\text {loc }}^{1}\left((0, T) \times \mathbb{R}^{2}\right), U_{ \pm} \in L_{\text {loc }}^{1}\left((0, T) \times \Omega_{ \pm}\right)$. Define the lower-order energy $E_{\mu}:(0, T] \rightarrow[0, \infty]$ for the $\mu$-approximate equations associated to the vector $\left(U_{+}, U_{-}, f\right)$, as follows:

$$
\begin{aligned}
E_{\mu}(t)= & E(t)+\mu^{2} \sum_{ \pm} \sum_{0 \leqq j \leqq 2} \underset{\tau \in(0, t)}{\operatorname{ess} \sup \left\|\partial_{t}^{j} \nabla u\right\|_{H^{3-j}\left(\Omega_{ \pm}\right)}^{2}} \\
& +\mu^{2} \sum_{ \pm} \int_{0}^{t}\left\|\partial_{t}^{3} u\right\|_{H^{1}\left(\Omega_{ \pm}\right)}^{2} \mathrm{~d} \tau \\
& +\mu \sum_{0 \leqq j \leqq 3} \int_{0}^{t}\left\|\partial_{t}^{j}(u \cdot n)\right\|_{H^{4-j}\left(\mathbb{R}^{2}\right)}^{2} \mathrm{~d} \tau \\
& +\mu^{2} \sum_{0 \leqq j \leqq 2} \operatorname{ess~sup}\left\|\partial_{t}^{j}(u \cdot n)\right\|_{H^{4.5-j}\left(\mathbb{R}^{2}\right)}^{2},
\end{aligned}
$$

where $E(t)$ denotes the energy for the fixed equations associated to $\left(U_{+}, U_{-}, f\right)$, as defined in Definition 15.

We define the initial lower-order energy $E_{\mu}^{0}$ for the $\mu$-approximate equations with lifting operator $L^{a}$ and right hand side $g$ associated to the initial data $\left(U_{+}^{0}, U_{-}^{0}, f^{0}\right)$ as

$$
\begin{aligned}
E_{\mu}^{0}= & E^{0}+\mu^{2} \sum_{ \pm} \sum_{j=0}^{2}\left\|\partial_{0}^{\mu, g, j} \nabla u^{0}\right\|_{H^{3-j}\left(\Omega_{ \pm}\right)} \\
& +\mu^{2} \sum_{0 \leqq j \leqq 2}\left\|\partial_{0}^{\mu, g, j}\left(u^{0} \cdot n^{0}\right)\right\|_{H^{4.5-j}\left(\mathbb{R}^{2}\right)}^{2}
\end{aligned}
$$

where in the definition of $E^{0}$ we replace $\partial_{0}^{j}$ with $\partial_{0}^{\mu, g, j}$. 
Definition 22. Let $f \in L_{\text {loc }}^{1}\left((0, T) \times \mathbb{R}^{2}\right), U_{ \pm} \in L_{\text {loc }}^{1}\left((0, T) \times \Omega_{ \pm}\right)$. Define the higher order energy $\mathcal{E}_{\mu}:(0, T] \rightarrow[0, \infty]$ associated with $\left(U_{+}, U_{-}, f\right)$ as follows:

$$
\begin{aligned}
\mathcal{E}_{\mu}(t)= & E_{\mu}(t)+\underset{\tau \in(0, t)}{\operatorname{ess} \sup } \sum_{ \pm} \sum_{0 \leqq j \leqq 3}\left\|\partial_{t}^{j} \nabla U\right\|_{H^{3-j}\left(\Omega_{ \pm}\right)}^{2}+\sum_{ \pm} \int_{0}^{t}\left\|\partial_{t}^{4} U\right\|_{L^{2}\left(\Omega_{ \pm}\right)}^{2} \mathrm{~d} \tau \\
& +\underset{\tau \in(0, t)}{\operatorname{ess} \sup } \sum_{ \pm} \sum_{0 \leqq j \leqq 2}\left\|\partial_{t}^{j}\left(\nabla^{\psi} \times u\right)\right\|_{H^{4-j}\left(\Omega_{ \pm}\right)}^{2} \\
& +\sum_{ \pm} \sum_{0 \leqq j \leqq 3} \int_{0}^{t}\left\|\partial_{t}^{j} \nabla u\right\|_{H^{4-j}\left(\Omega_{ \pm}\right)}^{2} \mathrm{~d} \tau \\
& +\sum_{0 \leqq j \leqq 3} \operatorname{ess~sup}\left\|\partial_{t}^{j} \nabla^{\prime} f\right\|_{H^{4.5-j}(\Gamma)}^{2}+\int_{0}^{t}\left\|\partial_{t}^{4} f\right\|_{H^{1}(\Gamma)}^{2} \mathrm{~d} \tau \\
& +\operatorname{ess~sup~}_{\tau \in(0, t)} \sum_{0 \leqq j \leqq 3}\left\|\partial_{t}^{j}(u \cdot n)\right\|_{H^{4-j}(\Gamma)}^{2}+\sum_{0 \leqq j \leqq 3} \int_{0}^{t}\left\|\partial_{t}^{j}(u \cdot n)\right\|_{H^{5.5-j}(\Gamma)}^{2} \mathrm{~d} \tau .
\end{aligned}
$$

Note that if $\mathcal{E}_{\mu}\left(T^{\prime}\right)<\infty$ then $\mathcal{E}_{\mu}$ is uniformly continuous on $\left(0, T^{\prime}\right]$.

We define the higher-order initial energy $\mathcal{E}_{\mu}^{0}$ for the $\mu$-approximate equations with lifting operator $L^{a}$ and right hand side $g$ associated to the initial data $\left(U_{+}^{0}, U_{-}^{0}, f^{0}\right)$ as

$$
\begin{aligned}
\mathcal{E}_{\mu}^{0}= & E_{\mu}^{0}+\sum_{ \pm} \sum_{0 \leqq j \leqq 3}\left\|\partial_{0}^{\mu, g, j} \nabla U^{0}\right\|_{H^{3-j}\left(\Omega_{ \pm}\right)}^{2} \\
& +\sum_{ \pm} \sum_{0 \leqq j \leqq 2}\left\|\partial_{0}^{\mu, g, j}\left(\nabla^{\psi^{0}} \times u^{0}\right)\right\|_{H^{4-j}\left(\Omega_{ \pm}\right)}^{2} \\
& +\sum_{0 \leqq j \leqq 3}\left\|\partial_{0}^{\mu, g, j} \nabla^{\prime} f^{0}\right\|_{H^{4.5-j}(\Gamma)}^{2}+\sum_{0 \leqq j \leqq 3}\left\|\partial_{0}^{\mu, g, j}\left(u^{0} \cdot n^{0}\right)\right\|_{H^{4-j}(\Gamma)}^{2}
\end{aligned}
$$

Note that we will use this energy for fixed $\mu$ when proving existence of solutions to the $\mu$-approximate equations and estimates involving it will always depend on $\mu$.

Definition 23. Given a right hand side function $g$ for shorthand we define the energy associated with $g$ as

$$
E_{g}(t):=\sum_{ \pm} \sum_{j=0}^{3} \underset{\tau \in(0, t)}{\operatorname{ess} \sup \|} \partial_{t}^{j} g_{1}\left\|_{H^{5-j}\left(\Omega_{ \pm}\right)}^{2}+\sum_{j=0}^{4} \underset{\tau \in(0, t)}{\operatorname{ess} \sup \| \partial_{t}^{j}} g_{2}\right\|_{H^{5-j}(\Gamma)}^{2}
$$

for $t \in(0,1]$. Note that if $E_{g}\left(T^{\prime}\right)<\infty$ then $E_{g}$ is uniformly continuous on $\left(0, T^{\prime}\right]$.

Note that for simplicity we have not attempted to make the order of $E_{g}(t)$ in space as low as possible since eventually $g$ will depend on the smoothed initial data for the original problem in the fixed domains. 
Definition 24. We say that initial data $\left(U_{+}^{0}, U_{-}^{0}, f^{0}\right)$ for the $\mu$-approximate equations is smooth if

$$
\mathcal{E}_{\max }^{0}<\infty
$$

where the high-order initial energy $\mathcal{E}_{\max }^{0}$ is defined as:

$$
\mathcal{E}_{\max }^{0}=\sum_{ \pm}\left\|U^{0}\right\|_{L^{\infty}\left(\Omega_{ \pm}\right)}^{2}+\left\|f^{0}\right\|_{L^{\infty}(\Gamma)}^{2}+\sum_{ \pm}\left\|\nabla U^{0}\right\|_{H^{20}\left(\Omega_{ \pm}\right)}+\left\|\nabla^{\prime} f^{0}\right\|_{H^{20}(\Gamma)}^{2}
$$

\subsection{Statement of Existence of Solutions to the $\mu$-Approximate Equations}

Theorem 3. Let $\mu \in(0,1]$ and let $\left(U_{+}^{0}, U_{-}^{0}, f^{0}\right)$ be smooth initial data for the $\mu$-approximate equations with lifting operator $L^{a}$ and right hand side $g$, with $\mathcal{E}_{\max }^{0}<\infty$. Assume that this initial data satisfies the compatibility condition (45) up to order 3 and the condition (46) up to order 2. Assume that the right hand side $g$ satisfies $E_{g}(1)<\infty$. Assume also that the initial data satisfy

$$
\begin{gathered}
\inf _{x \in \Omega_{ \pm}} \rho^{0}=: \delta^{0}>0 \\
\inf _{x \in \Omega_{ \pm}} \rho_{\mu}^{0}=: \delta_{\mu}^{0}>0 \\
\inf _{x \in \mathbb{R}^{3}} J^{\psi^{0}}=: \kappa^{0}>0 .
\end{gathered}
$$

Then there is a time $T_{\mu}^{0} \in(0,1]$ and a solution $\left(U_{+}, U_{-}, f\right)$ of the $\mu$ approximate equations with lifting operator $L^{a}$ and right hand side $g$ on the time interval $\left(0, T_{\mu}^{0}\right)$, as in Definition 18. Moreover,

$$
\begin{aligned}
& \mathcal{E}_{\mu}\left(T_{\mu}^{0}\right) \leqq C_{\mu}^{0}<\infty, \quad \rho \geqq \frac{\delta^{0}}{2} \quad \text { in }\left(0, T_{\mu}^{0}\right) \times \Omega_{ \pm}, \\
& \rho_{\mu} \geqq \frac{\delta_{\mu}^{0}}{2} \text { in }\left(0, T_{\mu}^{0}\right) \times \Omega_{ \pm}, \quad J^{\psi} \geqq \frac{1}{2} \kappa^{0} \quad \text { in } \quad\left(0, T_{\mu}^{0}\right) \times \mathbb{R}^{3}
\end{aligned}
$$

The constant $C_{\mu}^{0}>0$ is bounded above as follows:

$$
C_{\mu}^{0} \leqq C_{\mu}\left(\mathcal{E}_{\mu}^{0}, \frac{1}{\delta^{0}}, \frac{1}{\delta_{\mu}^{0}}, \frac{1}{\kappa^{0}}, E_{g}(1)\right)<\infty,
$$

where $C_{\mu}(\cdot)$ is a smooth increasing function of its arguments, and may depend on $\mu$.

The time $T_{\mu}^{0}>0$ is bounded below as follows:

$$
T_{\mu}^{0} \geqq T_{\mu}\left(\mathcal{E}_{\max }^{0}, \frac{1}{\delta^{0}}, \frac{1}{\delta_{\mu}^{0}}, \frac{1}{\kappa^{0}}, E_{g}(1)\right)>0,
$$

where $T_{\mu}(\cdot)$ is a smooth decreasing function of its arguments, and may depend on $\mu$. Note that the time interval of existence here depends on the high-order energy $\mathcal{E}_{\max }^{0}$ of the initial data.

Proof. See Sections 10-14. 
Theorem 4. Let $\mu \in(0,1]$ and let $\left(U_{+}^{0}, U_{-}^{0}, f^{0}\right)$ and $g$ satisfy the hypotheses of Theorem 3. Then there exists $T_{\mu} \in(0,1]$ and a solution $\left(U_{+}, U_{-}, f\right)$ of the $\mu$ approximate equations with lifting operator $L^{a}$ and right hand side $g$ on the time interval $\left(0, T_{\mu}\right)$, satisfying the following additional properties:

$$
\mathcal{E}_{\mu}(t)<\infty, \quad \inf _{\tau \in(0, t)} \inf _{x \in \Omega_{ \pm}} \rho>0, \quad \inf _{\tau \in(0, t)} \inf _{x \in \Omega_{ \pm}} \rho_{\mu}>0, \quad \inf _{\tau \in(0, t)} \inf _{x \in \mathbb{R}} J^{\psi}>0
$$

for all $t \in\left(0, T_{\mu}\right)$, and if $T_{\mu}<1$, then one of the following holds as $t \uparrow T_{\mu}$ :

$$
E_{\mu}(t) \rightarrow \infty, \quad \inf _{x \in \Omega_{ \pm}} \rho \rightarrow 0, \quad \inf _{x \in \Omega_{ \pm}} \rho_{\mu} \rightarrow 0, \quad \inf _{x \in \Omega_{ \pm}} J^{\psi} \rightarrow 0
$$

Note carefully that the blow-up condition is on the lower-order energy $E_{\mu}(t)$.

Proof. See Section 14.3.2.

8.5. Statement of the $\mu$-Independent Energy Estimate for the $\mu$-Approximate Equations

Proposition 7. Let $\left(U_{+}, U_{-}, f\right)$ be a solution of the $\mu$-approximate equations with lifting operator $L^{a}$ and right hand side $g$ as defined in Definition 18 on the time interval $(0, T)$ with initial data $\left(U_{+}^{0}, U_{-}^{0}, f^{0}\right)$, where $\mu \in(0,1]$. Suppose in addition that $\mathcal{E}_{\mu}(T)<\infty$ and that the initial data satisfies the conditions (49)-(51).

Then there exists a time $0<T^{0} \leqq 1$ and a constant $C^{0}>0$ such that

$$
\begin{aligned}
& E_{\mu}\left(\min \left\{T^{0}, T\right\}\right) \leqq C^{0}, \quad \rho_{ \pm} \geqq \frac{\delta^{0}}{2} \text { in }\left(0, \min \left\{T^{0}, T\right\}\right) \times \Omega_{ \pm}, \\
& \rho_{\mu \pm} \geqq \frac{\delta_{\mu}^{0}}{2} \quad \text { in } \quad\left(0, \min \left\{T^{0}, T\right\}\right) \times \Omega_{ \pm}, \quad J^{\psi} \geqq \frac{1}{2} \kappa^{0} \quad \text { in } \quad\left(0, \min \left\{T^{0}, T\right\}\right) \times \mathbb{R}^{3} .
\end{aligned}
$$

The time $T^{0}>0$ is bounded below as follows:

$$
T^{0} \geqq T\left(E_{\mu}^{0}, \frac{1}{\delta^{0}}, \frac{1}{\kappa^{0}}, \frac{1}{\delta_{\mu}^{0}}, E_{g}(1)\right)>0,
$$

where $T(\cdot)$ is a smooth decreasing function of its arguments. Similarly, the constant $C^{0}>0$ is bounded above as follows:

$$
C^{0} \leqq C\left(E_{\mu}^{0}, \frac{1}{\delta^{0}}, \frac{1}{\delta_{\mu}^{0}}, \frac{1}{\kappa^{0}}, E_{g}(1)\right)<\infty,
$$

where $C(\cdot)$ is a smooth increasing function of its arguments.

Proof. See Section 9. 


\subsection{Reduction to Solving the $\mu$-Approximate Equations}

Proposition 8. Let $\mu \in(0,1]$, let $\left(U_{+}^{0}, U_{-}^{0}, f^{0}\right)$ and $g$ satisfy the hypotheses of Theorem 3, and suppose that Theorem 4 and Proposition 7 hold. Then there exists $T^{0} \in(0,1]$ and a solution $\left(U_{+}, U_{-}, f\right)$ of the $\mu$-approximate equations with lifting operator $L^{a}$ and right hand side $g$ on the time interval $\left(0, T^{0}\right)$, satisfying the following additional properties:

$$
\begin{aligned}
& E_{\mu}\left(T^{0}\right) \leqq C^{0}, \quad \rho_{ \pm} \geqq \frac{\delta^{0}}{2} \quad \text { in } \quad\left(0, T^{0}\right) \times \Omega_{ \pm}, \\
& \rho_{\mu \pm} \geqq \frac{\delta_{\mu}^{0}}{2} \quad \text { in } \quad\left(0, T^{0}\right) \times \Omega_{ \pm}, \quad J^{\psi} \geqq \frac{1}{2} \kappa^{0} \quad \text { in } \quad\left(0, T^{0}\right) \times \mathbb{R}^{3} .
\end{aligned}
$$

The time $T^{0}>0$ is bounded below as follows:

$$
T^{0} \geqq T\left(E_{\mu}^{0}, \frac{1}{\delta^{0}}, \frac{1}{\kappa^{0}}, \frac{1}{\delta_{\mu}^{0}}, E_{g}(1)\right)>0,
$$

where $T(\cdot)$ is a smooth decreasing function of its arguments. Similarly, the constant $C^{0}>0$ is bounded above as follows:

$$
C^{0} \leqq C\left(E_{\mu}^{0}, \frac{1}{\delta^{0}}, \frac{1}{\delta_{\mu}^{0}}, \frac{1}{\kappa^{0}}, E_{g}(1)\right)<\infty,
$$

where $C(\cdot)$ is a smooth increasing function of its arguments.

Proof. This is straightforward using Theorem 4 and especially the blow-up criteria therein, together with the energy estimate given in Proposition 7.

Proposition 9. Let $\left(U_{+}^{0}, U_{-}^{0}, f^{0}\right)$ be smooth initial data for the original equations in the fixed domains satisfying the compatibility condition (32) up to order 2, and (31) up to order 3, plus the conditions

$$
\inf _{x \in \Omega_{ \pm}} \rho^{0}=: \delta^{0}>0, \quad \inf _{x \in \mathbb{R}^{3}} J^{\psi^{0}}=: \kappa^{0}>0 .
$$

Note that by being smooth we mean $\mathcal{E}_{\max }^{0}<\infty$ where $\mathcal{E}_{\max }^{0}$ is given in terms of $\left(U_{+}^{0}, U_{-}^{0}, f^{0}\right)$ by (48). Then there exists a $\mu^{0} \in(0,1]$ depending only on the initial density $\rho^{0}=\rho\left(p^{0}(x), s^{0}(x)\right)$ and functions $c_{1}(t, x), c_{2}(t, x)$ depending only on $\mu$ and the initial data $\left(U_{+}^{0}, U_{-}^{0}, f^{0}\right)$ which are smooth for $\rho^{0}, \rho_{\mu}^{0}, J^{\psi^{0}}>0$ such that the following is true. Firstly,

$$
\inf _{x \in \Omega_{ \pm}} \rho_{\mu}^{0}=: \delta_{\mu}^{0} \geqq \frac{\delta^{0}}{2}
$$

for all $\mu \in\left(0, \mu^{0}\right]$. Secondly,

$$
E_{c}(1) \rightarrow 0 \text { as } \mu \rightarrow 0
$$

where $c=\left(c_{1}, c_{2}\right)$ and $E_{c}$ is defined in (47) with c replacing $g$. Thirdly, the initial data $\left(U_{+}^{0}, U_{-}^{0}, f^{0}\right)$ satisfy the compatibility condition (46) up to order 2 , and (45) up to order 3 for the $\mu$-approximate equations with right hand side $c=\left(c_{1}, c_{2}\right)$. 
Proof. First of all, fix $\mu^{0} \in(0,1]$ such that $\rho_{\mu}^{0} \geqq \frac{\delta^{0}}{2}$ for all $\mu \in\left(0, \mu^{0}\right]$.

We define the functions $c_{1}$ and $c_{2}$ to make the initial data $\left(U_{+}^{0}, U_{-}^{0}, f^{0}\right)$ compatible with the $\mu$-approximate equations with right hand side $c=\left(c_{1}, c_{2}\right)$. We set

$$
c_{1 \pm}(t)=\sum_{k=0}^{2} \frac{t^{k}}{k !} c_{1 \pm}^{k} \quad \text { and } \quad c_{2}(t)=\sum_{k=0}^{2} \frac{t^{k}}{k !} c_{2}^{k}
$$

where the coefficients $c_{1 \pm}^{k}, c_{2}^{k}$ are defined below. Note that

$$
\begin{gathered}
\left.\partial_{t}^{j} c_{1 \pm}(t)\right|_{t=0}=c_{1 \pm}^{j} \text { for } 0 \leqq j \leqq 2 \\
\left.\partial_{t}^{j} c_{2}(t)\right|_{t=0}=c_{2}^{j} \text { for } 0 \leqq j \leqq 2 .
\end{gathered}
$$

We want to have, for $0 \leqq j \leqq 3$,

$$
\begin{aligned}
& \partial_{0}^{\mu, c, j} U_{ \pm}^{0}=\partial_{0}^{j} U_{ \pm}^{0} \\
& \partial_{0}^{\mu, c, j} f^{0}=\partial_{0}^{j} f^{0},
\end{aligned}
$$

where $\partial_{0}^{j}$ are the initial time derivatives associated with the equations on the fixed domains as defined in (13). We see that the equations defining $\partial_{0}^{\mu, c, j}$ are all the same as the equations defining $\partial_{0}^{j}$ except for the velocity equation (40). Let us assume inductively that (52)-(53) hold for $0 \leqq k \leqq j$ (and note the case $j=0$ is trivial). This means it makes sense for us to subtract the formula for $\partial_{0}^{j} u^{0}$ from the formula for $\partial_{0}^{\mu, c, j} u^{0}$ to obtain

$$
\begin{aligned}
\partial_{0}^{\mu, c, j+1} u_{ \pm}^{0}-\partial_{0}^{j+1} u_{ \pm}^{0} & \partial_{0}^{j}\left(\left(\frac{1}{\rho_{ \pm}^{0}}-\frac{1}{\rho_{\mu \pm}^{0}}\right) \nabla^{\psi^{0}} p_{ \pm}^{0}+\frac{\mu}{\rho_{\mu \pm}^{0}} \nabla^{\psi^{0}}\left(\rho_{ \pm}^{0}\left(c_{ \pm}^{0}\right)^{2} \nabla^{\psi^{0}} \cdot u_{ \pm}^{0}\right)\right) \\
& +\sum_{k+l=j, l \neq j} \partial_{0}^{k}\left(\frac{1}{\rho_{\mu \pm}^{0}}\right) c_{1 \pm}^{l}+\frac{1}{\rho_{\mu \pm}^{0}} c_{1 \pm}^{j} .
\end{aligned}
$$

Thus we define inductively, for $0 \leqq j \leqq 2$,

$$
\begin{aligned}
c_{1 \pm}^{j}= & -\rho_{\mu \pm}^{0} \sum_{k+l=j, l \neq j} \partial_{0}^{k}\left(\frac{1}{\rho_{\mu \pm}^{0}}\right) c_{1 \pm}^{l} \\
& -\rho_{\mu \pm}^{0} \partial_{0}^{j}\left(\left(\frac{1}{\rho_{ \pm}^{0}}-\frac{1}{\rho_{\mu \pm}^{0}}\right) \nabla^{\psi^{0}} p_{ \pm}^{0}+\frac{\mu}{\rho_{\mu \pm}^{0}} \nabla^{\psi^{0}}\left(\rho_{ \pm}^{0}\left(c_{ \pm}^{0}\right)^{2} \nabla^{\psi^{0}} \cdot u_{ \pm}^{0}\right)\right) .
\end{aligned}
$$

Hence (52)-(53) hold for $0 \leqq j \leqq 3$. Having established this, we will now write $\partial_{0}^{j}$ instead of $\partial_{0}^{\mu, c, j}$ for the rest of this proof where appropriate, when acting on this specific initial data $\left(U_{+}^{0}, U_{-}^{0}, f^{0}\right)$. 
We want the initial data $\left(U_{+}^{0}, U_{-}^{0}, f^{0}\right)$ to be compatible with the $\mu$-approximate equations, that is, to satisfy the compatibility condition (46) up to order 2 and the compatibility condition (45) up to order 3.

The fact that (45) holds up to order 3 for $\left(U_{+}^{0}, U_{-}^{0}, f^{0}\right)$ follows directly from the fact that (31) holds up to order 3 and (52)-(53) hold for $0 \leqq j \leqq 3$. This means $\partial_{0}^{j}\left(u_{+}^{0} \cdot n^{0}\right)=\partial_{0}^{j}\left(u_{-}^{0} \cdot n^{0}\right)$ for $0 \leqq j \leqq 3$ so we can write simply $\partial_{0}^{j}\left(u^{0} \cdot n^{0}\right)$. We define

$$
c_{2}^{j}:=-\mu\left(\Delta^{\prime}-1\right) \partial_{0}^{j}\left(u^{0} \cdot n^{0}\right)-\mu\left[\partial_{0}^{j}\left(\rho^{0}\left(c^{0}\right)^{2} \nabla^{\psi^{0}} \cdot u^{0}\right)\right] .
$$

Using the compatibility condition (32) for $0 \leqq j \leqq 2$, we have

$$
\begin{aligned}
\partial_{0}^{j} & {\left[p^{0}-\mu \rho^{0}\left(c^{0}\right)^{2} \nabla^{\psi^{0}} \cdot u^{0}\right]-\partial_{0}^{j}\left(-\sigma\left(\nabla^{\prime} \cdot \hat{n}^{0}\right)+\mu\left(\Delta^{\prime}-1\right)\left(\left.u^{0}\right|_{\Gamma} \cdot n^{0}\right)\right) } \\
& =-\partial_{0}^{j}\left[\mu \rho^{0}\left(c^{0}\right)^{2} \nabla^{\psi^{0}} \cdot u^{0}\right]-\partial_{0}^{j}\left(\mu\left(\Delta^{\prime}-1\right)\left(u^{0} \cdot n^{0}\right)\right) \\
& =c_{2}^{j},
\end{aligned}
$$

which is the compatibility condition (46). Thus (46) holds up to order 2.

It is now straightforward to show that $E_{c}(1) \rightarrow 0$ as $\mu \rightarrow 0$.

Proposition 10. Suppose that Theorem 4 and Proposition 7 are true.

Then the main theorem in the fixed domains, Theorem 2, follows, excluding the statement of uniqueness.

Proof. The proof is standard given Propositions 8 and 9, hence we sketch the details. We let $\left(U_{+}^{0}, U_{-}^{0}, f^{0}\right)$ be initial data for the original equations in the fixed domains satisfying the hypotheses therein plus the extra smoothness and compatibility allowed by Proposition 6 . Let $\mu^{0} \in(0,1]$ and $c^{\mu}=\left(c_{1}^{\mu}, c_{2}^{\mu}\right)$ for each $\mu \in\left(0, \mu^{0}\right]$ be given by Proposition 9 and use these as the right hand side of the $\mu$-approximate equations so the initial data are compatible.

Now it is quite straightforward using Proposition 8, and especially the energy estimate therein, together with weak-* compactness and Sovolev compact embedding, to let $\mu \rightarrow 0$ along a subsequence to obtain a solution of the equations in the fixed domains with the desired properties.

\subsection{The Curl of the Velocity Equation}

It will be necessary during the $\mu$-independent energy estimate to take the curl of the velocity equation. We will also introduce a linearised equation for the curl later, which is motivated by taking the curl of the velocity equation before linearisation. For these reasons we present the curl of the velocity equation, written as an equation for the curl of the velocity, $\nabla^{\psi} \times u$, below.

Lemma 3. Suppose that $u$ satisfies the $\mu$-approximate velocity equation (40). Suppose that in addition to the regularity stated in Definition 18 , we have $\partial_{t} \psi, \partial_{t} u, g_{1} \in$ $H_{\mathrm{loc}}^{1}\left(\Omega_{ \pm}\right), p, s, u, \nabla \psi, \nabla u \in H_{\mathrm{loc}}^{2}\left(\Omega_{ \pm}\right)$. Then $\nabla^{\psi} \times u$ satisfies the following equation.

$$
\rho_{\mu}\left(\partial_{t}+u^{\psi} \cdot \nabla\right)\left(\nabla^{\psi} \times u\right)=-\nabla^{\psi} \rho_{\mu} \times\left(\partial_{t}+u^{\psi} \cdot \nabla\right) u-\rho_{\mu}\left(\varepsilon_{i j k} \partial_{j}^{\psi} u_{l} \partial_{l}^{\psi} u_{k}\right)
$$




$$
+\nabla^{\psi} \times g_{1}
$$

in $(0, T) \times \Omega_{ \pm}$, where $\left(\varepsilon_{i j k} \partial_{j}^{\psi} u_{l} \partial_{l}^{\psi} u_{k}\right)$ denotes the vector with $i$-th component given by $\varepsilon_{i j k} \partial_{j}^{\psi} u_{l} \partial_{l}^{\psi} u_{k}$ and we have used summation convention for repeated indices.

In particular, our definition of initial time derivatives allows us to restrict the above equation to the time $t=0$ and replace $\partial_{t}$ with $\partial_{0}^{\mu, g, 1}$ etc.

Proof. We could apply $\nabla^{\psi} \times$ to the $\mu$-approximate velocity equation (40) directly, but it is easiest first to transform back to the original domains $\Omega_{ \pm}(t)$. We recall the velocity equation

$$
\rho_{\mu}\left(\partial_{t}+u^{\psi} \cdot \nabla\right) u+\nabla^{\psi} p=\mu \nabla^{\psi}\left(\rho c^{2} \nabla^{\psi} \cdot u\right)+g_{1}
$$

Setting $\check{p}=p \circ \theta^{-1}, \check{u}=u \circ \theta^{-1}$ and $\check{s}=s \circ \theta^{-1}, \check{\rho}_{\mu}=\rho_{\mu} \circ \theta^{-1}, \check{\rho}=\rho \circ \theta^{-1}$, $\check{c}=c \circ \theta^{-1}, \check{g}_{1}=g_{1} \circ \theta^{-1}$, where the diffeomorphism $\theta$ is defined in Lemma 1 , and using the coordinate change relations (35)-(36), we obtain

$$
\check{\rho}_{\mu}\left(\partial_{t}+\check{u} \cdot \nabla\right) \check{u}+\nabla \check{p}=\mu \nabla\left(\check{\rho} \check{c}^{2} \nabla \cdot \check{u}\right)+\check{g}_{1} .
$$

in $\Omega_{ \pm}^{T}$. Now we apply $\nabla \times$ to obtain

$$
\check{\rho}_{\mu}\left(\partial_{t}+\check{u} \cdot \nabla\right)(\nabla \times \check{u})+\left(\nabla \check{\rho}_{\mu}\right) \times\left(\partial_{t}+\check{u} \cdot \nabla\right) \check{u}+\check{\rho}_{\mu}\left(\varepsilon_{i j k} \partial_{x_{j}} \check{u}_{l} \partial_{x_{l}} \check{u}_{k}\right)=\nabla \times \check{g}_{1} .
$$

Then we use the coordinate change relations (35)-(36) again to obtain

$$
\begin{aligned}
& \rho_{\mu}\left(\partial_{t}+u^{\psi} \cdot \nabla\right)\left(\nabla^{\psi} \times u\right)+\left(\nabla^{\psi} \rho_{\mu}\right) \times\left(\partial_{t}+u^{\psi} \cdot \nabla\right) u+\rho_{\mu}\left(\varepsilon_{i j k} \partial_{j}^{\psi} u_{l} \partial_{l}^{\psi} u_{k}\right) \\
& \quad=\nabla^{\psi} \times g_{1}
\end{aligned}
$$

as required.

\section{Proof of the $\mu$-Independent Energy Estimate for the $\mu$-Approximate Equations}

In this section we assume we are given a solution $\left(U_{+}, U_{-}, f\right)$ of the $\mu$ approximate Equations (39)-(44) satisfying the hypotheses of Proposition 7 and we proceed to show that the conclusions of Proposition 7 hold.

\subsection{Notation for Constants and Functions of the Energy and Initial Data}

Definition 25. It will be convenient to define some generic constants/functions depending on the energy and initial data in a given way.

Firstly, we will always write $F$ for a generic smooth increasing function of its arguments which is independent of $\mu$.

We will write $C$ for a generic constant which is independent of the solution, including being independent of the initial data, and independent of $\mu$. 
We define $M^{0}$ as a generic constant of the form

$$
M^{0}:=F\left(E_{\mu}^{0}, \frac{1}{\delta^{0}}, \frac{1}{\delta_{\mu}^{0}}, \frac{1}{\kappa^{0}}, E_{g}(1)\right)
$$

We define $K(t)$ as a generic function of $t$ of the form

$$
K(t):=F\left(E_{\mu}(t), M^{0}\right)
$$

Remark 3. Note that for $t$ bounded above, we may bound the square of the expression $M^{0}+t K(t)$ by an expression of the same form. In fact, since we are considering short-time existence only, we have chosen to always bound $t$ above by 1 , where 1 is chosen as a convenient strictly positive constant.

Note that throughout this section we will group lower order terms into generic remainder terms, denoted by $R$ with appropriate superscripts and subscripts to denote the order of the remainder. We define these remainder terms and prove estimates for them in Section 9.18 in order to try and focus on the more important estimates in the main body of the proof of the $\mu$-independent energy estimate.

\subsection{The Material Derivative Operators}

Definition 26. We define the material derivative operators $D_{t}^{u_{ \pm}}$by

$$
D_{t}^{u_{ \pm}}=\partial_{t}+u_{ \pm}^{\psi} \cdot \nabla
$$

It is important to note that we allow $D_{t}^{u_{-}}$to act on functions on $\Omega_{+}$by defining $u_{-}$on $\Omega_{+}$by Sobolev extension, and vice-versa, in which case $D_{t}^{u_{-}}$(or $D_{t}^{u_{+}}$) is no longer a material derivative in $\Omega_{+}$(or $\Omega_{-}$), but it has the important advantage of being continuous across the interface $\Gamma$.

Note that $D_{t}^{u_{ \pm}}$acts along $\Gamma$ since

$$
D_{t}^{u_{ \pm}}=\partial_{t}+u_{ \pm}^{\prime} \cdot \nabla^{\prime}
$$

on $\Gamma$ as $u_{3 \pm}^{\psi}=0$ on $\Gamma$.

We also define the material derivative of the pressure $\dot{p}$ by

$$
\dot{p}_{ \pm}=D_{t}^{u \pm} p_{ \pm}
$$

Note that, by virtue of the pressure equation (39) which is the same as (25), we have the important relation

$$
\dot{p}=-\rho c^{2} \nabla^{\psi} \cdot u
$$

for solutions of the equations. 


\subsection{Restriction of the Time Interval}

Note that by uniform continuity and the conditions (49)-(51), there exists a $0<\tilde{T} \leqq \min \{T, 1\}$, which may depend on the solution, such that

$$
\begin{aligned}
\rho & \geqq \frac{1}{2} \delta^{0} \\
\rho_{\mu} & \geqq \frac{1}{2} \delta_{\mu}^{0} \\
J^{\psi} & \geqq \frac{1}{2} \kappa^{0}
\end{aligned}
$$

for $t \in(0, \tilde{T})$.

We define $T^{\prime}$ to be the supremum over all such $\tilde{T}$.

In the estimates below will assume that $t \in\left(0, T^{\prime}\right)$ until stated otherwise. Part of our goal will be to prove that $T^{\prime}$ can be taken bigger than $T^{0}$, which depends only on the initial data.

Our main aim will be to show that for $t \in\left(0, T^{\prime}\right)$ we have

$$
E_{\mu}(t) \leqq\left(M^{0}+t K(t)\right)\left(\frac{1}{\varepsilon^{\frac{3}{2}}}+\varepsilon^{\frac{1}{2}} E_{\mu}(t)\right),
$$

where we are free to choose $\varepsilon \in(0,1]$. We could absorb the term $\varepsilon^{\frac{1}{2}} t K(t) E_{\mu}(t)$ into the term $\frac{1}{\varepsilon^{\frac{3}{2}}} t K(t)$ but because this is how the estimate naturally comes out, and also to maintain consistency with later similar notation, we leave it like this.

\subsection{Using the Energy to Extend the Assumptions on the Initial Data}

We claim that the following inequalities hold:

$$
\begin{aligned}
\left\|\rho-\rho^{0}\right\|_{L^{\infty}\left(\Omega_{ \pm}\right)} & \leqq t K(t) \\
\left\|\rho_{\mu}-\rho_{\mu}^{0}\right\|_{L^{\infty}\left(\Omega_{ \pm}\right)} & \leqq t K(t) \\
\left\|\partial_{x_{3}} \psi-\partial_{x_{3}} \psi^{0}\right\|_{L^{\infty}\left(\Omega_{ \pm}\right)} & \leqq t K(t) .
\end{aligned}
$$

This is easy to check using the Sobolev embedding theorem $H^{2}\left(\Omega_{ \pm}\right) \subset L^{\infty}\left(\Omega_{ \pm}\right)$ and the fundamental theorem of calculus.

\subsection{Estimate of the Lower Order Terms in the Energy}

We claim that

$$
\begin{aligned}
\|U, \psi\|_{L^{\infty}\left(\Omega_{ \pm}\right)} & \leqq M^{0}+t K(t) \\
\|f\|_{W^{1, \infty}(\Gamma)} & \leqq M^{0}+t K(t) \\
\left\|\partial^{\alpha} U\right\|_{L^{2}\left(\Omega_{ \pm}\right)}^{2} & \leqq M^{0}+t K(t) \\
\left\|\partial^{\alpha} f\right\|_{H^{1}(\Gamma)}^{2}+\left\|\partial^{\beta} f\right\|_{H^{2.5}(\Gamma)}^{2} & \leqq M^{0}+t K(t),
\end{aligned}
$$

where $1 \leqq|\alpha| \leqq 2$ and $|\beta|=1$. Indeed, this is easy to check using the fundamental theorem of calculus and the Sobolev embedding theorem for the first two inequalities. 


\subsection{The Differentiated Equations and the Corrected Pressure, Velocity and} Entropy

Once we have differentiated the equations enough times, the lower order terms may all be estimated in terms of the energy by the Sobolev embedding theorem, and hence we may try to obtain energy estimates in the same way as for constant-coefficient linear equations. One problem is that the pressure and velocity equations involve a time derivative of the lift of the front, $\psi$, whose time derivatives can only be estimated to the same order as the velocity etc, so it cannot be treated as a coefficient. Treating $\psi$ like the other unknowns $U$ makes the equations non-symmetric so that we cannot obtain energy estimates directly. To rectify this, we introduce corrected pressure, velocity and entropy functions-introduced in the linear setting by AlinHaC [2] and referred to as the good unknownswhich absorbs the highest order derivatives of $\psi$ into the unknowns. Note that we only need to use these when estimating the highest order time derivatives, although we may use them for estimating other derivatives as well simply for convenience.

9.6.1. The Differentiated Interior Equations We apply $\partial^{\alpha}$ to the equations (39)-(41), where $1 \leqq|\alpha| \leqq 3$, and use the Leibniz and chain rules to obtain the following equations (note that, prior to differentiation, we have replaced $\rho c^{2} \nabla^{\psi} \cdot u$ with $-\dot{p}$, which is more convenient to work with):

$$
\begin{aligned}
& \frac{1}{\rho c^{2}}\left(\partial_{t}+u^{\psi} \cdot \nabla\right) \partial^{\alpha} p \\
& c-\frac{1}{\rho c^{2} J^{\psi}}\left(\partial_{t} \partial^{\alpha} \psi+u \cdot \nabla \partial^{\alpha} \psi-\frac{1}{J^{\psi}}\left(\partial_{x_{3}} \partial^{\alpha} \psi\right)\left(\partial_{t} \psi+u \cdot \nabla \psi\right)\right) \partial_{x_{3}} p \\
& \quad+\nabla^{\psi} \cdot \partial^{\alpha} u-\frac{\nabla \partial^{\alpha} \psi}{J^{\psi}} \cdot \partial_{x_{3}} u+\frac{\nabla \psi}{\left(J^{\psi}\right)^{2}} \partial_{x_{3}} \partial^{\alpha} \psi \cdot \partial_{x_{3}} u=R^{|\alpha|}(U, \psi) \\
& \rho_{\mu}\left(\partial_{t}+u^{\psi} \cdot \nabla\right) \partial^{\alpha} u-\frac{\rho_{\mu}}{J^{\psi}}\left(\partial_{t} \partial^{\alpha} \psi+u \cdot \nabla \partial^{\alpha} \psi-\frac{1}{J^{\psi}}\left(\partial_{x_{3}} \partial^{\alpha} \psi\right)\left(\partial_{t} \psi\right.\right. \\
& \quad+u \cdot \nabla \psi)) \partial_{x_{3}} u+\nabla^{\psi} \partial^{\alpha} p-\frac{\nabla \partial^{\alpha} \psi}{J^{\psi}} \partial_{x_{3}} p+\frac{\nabla \psi}{\left(J^{\psi}\right)^{2}} \partial_{x_{3}} \partial^{\alpha} \psi \partial_{x_{3}} p \\
& =-\mu \nabla^{\psi} \partial^{\alpha} \dot{p}+R^{|\alpha|}(U, \psi)+\mu R^{|\alpha|}(U, \psi, \dot{p}) \\
& \left(\partial_{t}+u^{\psi} \cdot \nabla\right) \partial^{\alpha} s-\frac{1}{J^{\psi}}\left(\partial_{t} \partial^{\alpha} \psi+u \cdot \nabla \partial^{\alpha} \psi-\frac{1}{J^{\psi}}\left(\partial_{x_{3}} \partial^{\alpha} \psi\right)\left(\partial_{t} \psi+u \cdot \nabla \psi\right)\right) \partial_{x_{3}} s \\
& =R^{|\alpha|}(U, \psi)
\end{aligned}
$$

in $\left(0, T^{\prime}\right) \times \Omega_{ \pm}$. The lower order remainder terms on the right hand side are defined in Definitions 28 and 29. Note that in fact, we could absorb the terms involving $\nabla \partial^{\alpha} \psi$ into the remainder, but not the terms involving $\partial_{t} \partial^{\alpha} \psi$ if $\partial^{\alpha}$ consists of all time derivatives.

Indeed, we now assume that $\alpha_{0} \leqq 2$. Because $\partial^{\alpha}$ contains a maximum of 2 time derivatives, we may absorb the terms involving high-order derivatives of $\psi$ in 
the Equations (66)-(67) into the remainder terms. Hence we obtain the following equations:

$$
\begin{gathered}
\frac{1}{\rho c^{2}}\left(\partial_{t}+u^{\psi} \cdot \nabla\right) \partial^{\alpha} p+\nabla^{\psi} \cdot \partial^{\alpha} u=R^{|\alpha|}(U, \psi) \\
\rho_{\mu}\left(\partial_{t}+u^{\psi} \cdot \nabla\right) \partial^{\alpha} u+\nabla^{\psi} \partial^{\alpha} p+\mu \nabla^{\psi} \partial^{\alpha} \dot{p}=R^{|\alpha|}(U, \psi)+\mu R^{|\alpha|}(U, \psi, \dot{p})
\end{gathered}
$$

$$
\left(\partial_{t}+u^{\psi} \cdot \nabla\right) \partial^{\alpha} s=R^{|\alpha|}(U, \psi)
$$

in $\left(0, T^{\prime}\right) \times \Omega_{ \pm}$.

9.6.2. The Corrected Pressure, Velocity and Entropy For $\alpha$ a multi-index with $2 \leqq|\alpha| \leqq 3$, we define the corrected unknowns (pressure, velocity and entropy) as

$$
U^{\alpha}=\partial^{\alpha} U-\frac{\partial^{\alpha} \psi}{J^{\psi}} \partial_{x_{3}} U
$$

We write

$$
U^{\alpha}=\left(p^{\alpha}, u^{\alpha}, s^{\alpha}\right)
$$

Note that, provided $|\alpha|$ is large enough, $\partial U^{\alpha}=\partial \partial^{\alpha} U-\frac{\partial \partial^{\alpha} \psi}{J \psi} \partial_{x_{3}} U$ plus lower order terms, and we see exactly this term in the differentiated pressure equations. This is the motivation for the definition of $U^{\alpha}$.

Lemma 4. For $2 \leqq|\alpha| \leqq 3$ and $|\beta|=2$, we have

$$
\begin{aligned}
\left\|\partial^{\alpha} U\right\|_{L^{2}\left(\Omega_{ \pm}\right)}^{2} & \leqq C\left\|U^{\alpha}\right\|_{L^{2}\left(\Omega_{ \pm}\right)}^{2}+\left(M^{0}+t K(t)\right)\left\|\partial^{\alpha} f\right\|_{H^{1}(\Gamma)}^{2} \\
\left\|D_{t}^{u-} \partial^{\beta} U\right\|_{L^{2}\left(\Omega_{ \pm}\right)}^{u_{-}} & \leqq C\left\|D_{t}^{u_{-}} U^{\beta}\right\|_{L^{2}\left(\Omega_{ \pm}\right)}^{2}+\left(M^{0}+t K(t)\right)\left\|\left(\partial^{\beta} f, \partial^{\prime} \partial^{\beta} f\right)\right\|_{H^{1}(\Gamma)}^{2} \\
\left\|U^{\alpha}\right\|_{L^{2}\left(\Omega_{ \pm}\right)}^{2} & \leqq C\left\|\partial^{\alpha} U\right\|_{L^{2}\left(\Omega_{ \pm}\right)}^{2}+\left(M^{0}+t K(t)\right)\left\|\partial^{\alpha} f\right\|_{H^{1}(\Gamma)}^{2} .
\end{aligned}
$$

Proof. This is easy to check using the lower order estimates (62)-(64) and the Sobolev embedding theorem plus Holder's inequality to estimate the $L^{2}$ norm of a product in terms of the product of $H^{1}$ norms where necessary.

Lemma 5. For $2 \leqq|\alpha| \leqq 3$, we have

$$
\begin{aligned}
\left(\partial_{t}+u^{\psi} \cdot \nabla\right) \partial^{\alpha} U= & \left(\partial_{t}+u^{\psi} \cdot \nabla\right) U^{\alpha}+\frac{\left(\partial_{t}+u^{\psi} \cdot \nabla\right) \partial^{\alpha} \psi}{J^{\psi}} \partial_{x_{3}} U+R^{|\alpha|}(U, \psi) \\
= & \left(\partial_{t}+u^{\psi} \cdot \nabla\right) U^{\alpha}+\frac{1}{J^{\psi}}\left(\left(\partial_{t}+u \cdot \nabla\right) \partial^{\alpha} \psi\right. \\
& \left.-\frac{1}{J^{\psi}}\left(\partial_{t} \psi+u \cdot \nabla \psi\right) \partial_{x_{3}} \partial^{\alpha} \psi\right) \partial_{x_{3}} U \\
& +R^{|\alpha|}(U, \psi)
\end{aligned}
$$


and

$$
\begin{aligned}
\nabla^{\psi} \partial^{\alpha} U & =\nabla^{\psi} U^{\alpha}+\frac{\nabla^{\psi} \partial^{\alpha} \psi}{J^{\psi}} \partial_{x_{3}} U+R^{|\alpha|}(U, \psi) \\
& =\nabla^{\psi} U^{\alpha}+\frac{1}{J \psi}\left(\nabla \partial^{\alpha} \psi-\frac{\nabla \psi}{J^{\psi}} \partial_{x_{3}} \partial^{\alpha} \psi\right) \partial_{x_{3}} U+R^{|\alpha|}(U, \psi),
\end{aligned}
$$

where $R^{k}(U, \psi)$ denotes the same generic remainder term given in Definition 28.

Proof. We may simply differentiate the formula (72) to find

$$
\partial \partial^{\alpha} U=\partial U^{\alpha}+\frac{\partial \partial^{\alpha} \psi}{J^{\psi}} \partial_{x_{3}} U+\frac{\partial^{\alpha} \psi}{J^{\psi}} \partial \partial_{x_{3}} U-\frac{\partial^{\alpha} \psi}{\left(J^{\psi}\right)^{2}} \partial \partial_{x_{3}} \psi \partial_{x_{3}} U,
$$

and we note that the last two terms on the right have the same form as the remainder $R^{|\alpha|}(U, \psi)$, provided that $2 \leqq|\alpha| \leqq 3$. Then we simply use the definitions of $\nabla^{\psi}$ and $u^{\psi}$.

We now substitute

$$
\partial^{\alpha} U=U^{\alpha}+\frac{\partial^{\alpha} \psi}{J^{\psi}} \partial_{x_{3}} U
$$

in the Equations (66)-(68) for $2 \leqq|\alpha| \leqq 3$ and use the above lemma to obtain

$$
\begin{aligned}
\frac{1}{\rho c^{2}}\left(\partial_{t}+u^{\psi} \cdot \nabla\right) p^{\alpha}+\nabla^{\psi} \cdot u^{\alpha} & =R^{|\alpha|}(U, \psi) \\
\rho_{\mu}\left(\partial_{t}+u^{\psi} \cdot \nabla\right) u^{\alpha}+\nabla^{\psi} p^{\alpha} & =-\mu \nabla^{\psi} \partial^{\alpha} \dot{p}+R^{|\alpha|}(U, \psi)+\mu R^{|\alpha|}(U, \psi, \dot{p}) \\
\left(\partial_{t}+u^{\psi} \cdot \nabla\right) s^{\alpha} & =R^{|\alpha|}(U, \psi)
\end{aligned}
$$

in $\left(0, T^{\prime}\right) \times \Omega_{ \pm}$.

9.6.3. Tangential and Time Derivatives of the Jump Conditions We may differentiate the jump conditions (42)-(43) with respect to time and the horizontal directions to obtain

$$
\begin{aligned}
\partial_{t} \partial^{\alpha} f & =\partial^{\alpha} u_{ \pm} \cdot n-u_{ \pm}^{\prime} \cdot \nabla^{\prime} \partial^{\alpha} f+R_{\Gamma}^{|\alpha|-1}(U, f) \\
{\left[\partial^{\alpha} p+\mu \partial^{\alpha} \dot{p}\right] } & =-\sigma \nabla^{\prime} \cdot \partial^{\alpha} \hat{n}+\mu\left(\Delta^{\prime}-1\right) \partial^{\alpha}(u \cdot n)+\partial^{\alpha} g_{2}
\end{aligned}
$$

on $\left(0, T^{\prime}\right) \times \Gamma$, where $\alpha$ is a multi-index with $|\alpha| \leqq 3$ and $\alpha_{3}=0$. We find it convenient not to try and write these in terms of the corrected pressure and velocity for the time being. The lower order remainder terms on the right hand side are defined in Definition 30. 


\subsubsection{Differentiating the Unit Normal}

Lemma 6. Let $1 \leqq|\alpha| \leqq 3$ with $\alpha_{3}=0$. Then

$$
\partial^{\alpha}\left(\frac{\nabla^{\prime} f}{|n|}\right)=\frac{\partial^{\alpha} \nabla^{\prime} f}{|n|}-\frac{\left(\nabla^{\prime} f \cdot \partial^{\alpha} \nabla^{\prime} f\right) \nabla^{\prime} f}{|n|^{3}}+R_{\Gamma}^{|\alpha|-1}(f)
$$

where the remainder $R_{\Gamma}^{|\alpha|-1}(f)$ is defined in Definition 31.

Proof. This is a simple application of the product rule.

Lemma 7. Let $w \in \mathbb{R}^{2}$ be any vector (in particular we are interested in $w=$ $\left.\partial^{\alpha} \nabla^{\prime} f\right)$. Then

$$
\frac{1}{|n|^{3}}|w|^{2} \leqq \frac{1}{|n|}|w|^{2}-\frac{1}{|n|^{3}}\left|\nabla^{\prime} f \cdot w\right|^{2}
$$

Proof. This is a simple application of Cauchy-Schwarz.

\subsection{A Slightly Lower Order Estimate for the Front}

Let $|\alpha|=3$ with $\alpha_{3}=0$. In this section we wish to estimate $\partial^{\alpha} f$ as opposed to $\partial^{\alpha} \nabla^{\prime} f$.

Suppose $\partial^{\alpha}=\partial_{x_{i}} \partial^{\beta}$ for $|\beta|=2$. Then in fact

$$
\left\|\partial^{\alpha} f\right\|_{L^{2}(\Gamma)}^{2} \leqq\left\|\partial^{\beta} \nabla^{\prime} f\right\|_{L^{2}(\Gamma)}^{2} \leqq M^{0}+t K(t),
$$

where we have used the lower order estimate (65).

Otherwise, $\partial^{\alpha}=\partial_{t} \partial^{\beta}$ for $|\beta|=2$. Then the Equation (79) implies

$$
\partial_{t} \partial^{\beta} f=\partial^{\beta} u \cdot n-u^{\prime} \cdot \partial^{\beta} \nabla^{\prime} f+R_{\Gamma}^{1}(U, f) .
$$

Hence, using the lower order estimates (62)-(65) and the lower order remainder estimate (122), we have

$$
\begin{aligned}
\left\|\partial_{t} \partial^{\beta} f\right\|_{L^{2}(\Gamma)}^{2} & \leqq\left(M^{0}+t K(t)\right)\left(1+\left\|\partial^{\beta} u\right\|_{L^{2}(\Gamma)}^{2}\right) \\
& \leqq\left(M^{0}+t K(t)\right)\left(1+\varepsilon\left\|\partial^{\beta} u\right\|_{H^{1}\left(\Omega_{-}\right)}^{2}+\frac{1}{\varepsilon}\left\|\partial^{\beta} u\right\|_{L^{2}\left(\Omega_{-}\right)}^{2}\right) \\
& \leqq\left(M^{0}+t K(t)\right)\left(\frac{1}{\varepsilon}+\varepsilon E_{\mu}(t)\right),
\end{aligned}
$$

where we have used the lower order estimate (64) in the last line.

Hence we have shown that

$$
\sum_{|\alpha|=3}\left\|\partial^{\alpha} f\right\|_{L^{2}(\Gamma)}^{2} \leqq\left(M^{0}+t K(t)\right)\left(\frac{1}{\varepsilon}+\varepsilon E_{\mu}(t)\right) .
$$




\subsection{Estimate of the Entropy}

We multiply Equation (78) by $s^{\alpha}$, where $2 \leqq|\alpha| \leqq 3$, and use the product rule to obtain

$$
\begin{aligned}
\frac{1}{2} \partial_{t}\left|s^{\alpha}\right|^{2}+\frac{1}{2} \nabla \cdot\left(u^{\psi}\left|s^{\alpha}\right|^{2}\right) & =\frac{1}{2}\left(\nabla \cdot u^{\psi}\right)\left|s^{\alpha}\right|^{2}+R^{|\alpha|}(U, \psi) s^{\alpha} \\
& =R^{|\alpha|}(U, \psi) s^{\alpha}
\end{aligned}
$$

in $\left(0, T^{\prime}\right) \times \Omega_{ \pm}$. Note that we have absorbed the lower-order term generated by the product rule into the remainder $R^{|\alpha|}(U, \psi)$ on the right hand side. Then we integrate over $\Omega_{ \pm}$and apply the divergence theorem for Sobolev functions to the second term on the left. Note that since $\left(u^{\psi}\right)_{3 \pm}=0$ on $\Gamma$ we are left with no boundary terms. Hence we obtain

$$
\frac{1}{2} \frac{\mathrm{d}}{\mathrm{d} t} \int_{\Omega_{ \pm}}\left|s^{\alpha}\right|^{2} \mathrm{~d} x=\int_{\Omega_{ \pm}} R^{|\alpha|}(U, \psi) s^{\alpha} \mathrm{d} x .
$$

Applying Cauchy's inequality to the right hand side, we obtain

$$
\frac{\mathrm{d}}{\mathrm{d} t}\left\|s^{\alpha}\right\|_{L^{2}\left(\Omega_{ \pm}\right)}^{2} \leqq\left\|R^{|\alpha|}(U, \psi)\right\|_{L^{2}\left(\Omega_{ \pm}\right)}^{2}+\left\|s^{\alpha}\right\|_{L^{2}\left(\Omega_{ \pm}\right)}^{2} .
$$

Then using the estimate of the remainder given by lemma 118, and the estimate of $s^{\alpha}$ from (73), then integrating from 0 to $t$, we obtain

$$
\left\|s^{\alpha}\right\|_{L^{2}\left(\Omega_{ \pm}\right)}^{2} \leqq M^{0}+t K(t)
$$

\subsection{Estimate of the Tangential Derivatives of the Pressure, Velocity and Front}

Here we come to one of the main parts of the energy estimate. Let $\alpha$ be a multiindex with $|\alpha|=3$ and $\alpha_{3}=0$, so that $\partial^{\alpha}$ contains no normal derivatives. Assume also that $\alpha_{0} \leqq 2$, so that we may, if we wish, write $\partial^{\alpha}=\partial_{x_{i}} \partial^{\beta}$ for some $1 \leqq i \leqq 2$ and $|\beta|=2$.

We intend to multiply the differentiated pressure and velocity equations (69)(70) by $\partial^{\alpha} p$ and $\partial^{\alpha} u$ respectively, then integrate by parts to obtain an energy estimate as for the entropy. Unfortunately, since there are normal derivatives in these equations, we will generate a boundary term on the interface. To deal with this boundary term we will need to use the pressure interface condition (43) and the equation (42) for the front. Note that we have differentiated these in the tangential and time directions to obtain (80) and (79), but we cannot do the same for the normal derivatives. Hence we will have to estimate these separately. In this section we exclude the estimate of the highest order time derivatives since the surface tension regularises the front in space, but not in time.

We multiply (69) by $\partial^{\alpha} p$ and (70) by $\partial^{\alpha} u$. We then add the resulting equations to obtain

$$
\frac{1}{\rho c^{2}}\left(\left(\partial_{t}+u^{\psi} \cdot \nabla\right) \partial^{\alpha} p\right) \partial^{\alpha} p+\rho_{\mu}\left(\left(\partial_{t}+u^{\psi} \cdot \nabla\right) \partial^{\alpha} u\right) \cdot \partial^{\alpha} u+\left(\nabla^{\psi} \cdot \partial^{\alpha} u\right) \partial^{\alpha} p
$$




$$
\begin{aligned}
& +\left(\nabla^{\psi} \partial^{\alpha} p\right) \cdot \partial^{\alpha} u=-\mu \nabla^{\psi} \partial^{\alpha} \dot{p} \cdot \partial^{\alpha} u+R^{3}(U, \psi) \partial^{\alpha} p+\left(R^{3}(U, \psi)\right. \\
& \left.+\mu R^{3}(U, \psi, \dot{p})\right) \cdot \partial^{\alpha} u
\end{aligned}
$$

in $\left(0, T^{\prime}\right) \times \Omega_{ \pm}$. Now we use the product rule to obtain

$$
\begin{aligned}
& \frac{1}{2} \partial_{t}\left(\frac{1}{\rho c^{2}}\left|\partial^{\alpha} p\right|^{2}\right)+\frac{1}{2} \partial_{t}\left(\rho_{\mu}\left|\partial^{\alpha} u\right|^{2}\right)+\frac{1}{2} \nabla \cdot\left(\frac{1}{\rho c^{2}} u^{\psi}\left|\partial^{\alpha} p\right|^{2}\right) \\
& \quad+\frac{1}{2} \nabla \cdot\left(\rho_{\mu} u^{\psi}\left|\partial^{\alpha} u\right|^{2}\right) \\
& \quad+\nabla^{\prime} \cdot\left(\partial^{\alpha} p \partial^{\alpha} u\right)+\partial_{x_{3}}\left(\frac{\left(-\nabla^{\prime} \psi, 1\right)}{J^{\psi}} \partial^{\alpha} p \cdot \partial^{\alpha} u\right) \\
& \quad+\mu\left(\nabla^{\prime} \partial^{\alpha} \dot{p}+\partial_{x_{3}}\left(\frac{\left(-\nabla^{\prime} \psi, 1\right)}{J^{\psi}} \partial^{\alpha} \dot{p}\right)\right) \cdot \partial^{\alpha} u \\
& =R^{3}(U, \psi) \partial^{\alpha} p+R^{3}(U, \psi) \cdot \partial^{\alpha} u+\mu R^{3}(U, \psi, \dot{p}) \cdot \partial^{\alpha} u
\end{aligned}
$$

where we have absorbed some lower order terms into the remainder on the right hand side. Now we integrate over $\Omega_{ \pm}$and apply the divergence theorem. Note that the outward unit normal to $\Gamma$ in $\Omega_{ \pm}$is $(0,0, \mp 1)$. The terms which are tangential divergences vanish, and $\left(u^{\psi}\right)_{3 \pm}=0$ on $\Gamma$ hence the third and fourth terms vanish. We obtain

$$
\begin{aligned}
& \frac{\mathrm{d}}{\mathrm{d} t} \int_{\Omega_{ \pm}} \frac{1}{\rho c^{2}}\left|\partial^{\alpha} p\right|^{2} \mathrm{~d} x+\frac{\mathrm{d}}{\mathrm{d} t} \int_{\Omega_{ \pm}} \rho_{\mu}\left|\partial^{\alpha} u\right|^{2} \mathrm{~d} x \mp 2 \int_{\Gamma}\left(\partial^{\alpha} p+\mu \partial^{\alpha} \dot{p}\right) \partial^{\alpha} u \cdot n \mathrm{~d} x^{\prime} \\
& \quad-\mu \int_{\Omega_{ \pm}} \partial^{\alpha} \dot{p} \nabla^{\psi} \cdot \partial^{\alpha} u \mathrm{~d} x=\int_{\Omega_{ \pm}} R^{3}(U, \psi) \partial^{\alpha} p+\left(R^{3}(U, \psi)\right. \\
& \left.\quad+\mu R^{3}(U, \psi, \dot{p})\right) \cdot \partial^{\alpha} u \mathrm{~d} x .
\end{aligned}
$$

We note that

$$
\partial^{\alpha} \dot{p}=-\partial^{\alpha}\left(\rho c^{2} \nabla^{\psi} \cdot u\right)=-\rho c^{2} \nabla^{\psi} \cdot \partial^{\alpha} u+R^{3}(U, \psi) .
$$

Hence

$$
\begin{aligned}
\frac{\mathrm{d}}{\mathrm{d} t} \int_{\Omega_{ \pm}} \frac{1}{\rho c^{2}}\left|\partial^{\alpha} p\right|^{2} \mathrm{~d} x+\frac{\mathrm{d}}{\mathrm{d} t} \int_{\Omega_{ \pm}} \rho_{\mu}\left|\partial^{\alpha} u\right|^{2} \mathrm{~d} x \mp 2 \int_{\Gamma}\left(\partial^{\alpha} p+\mu \partial^{\alpha} \dot{p}\right) \partial^{\alpha} u \cdot n \mathrm{~d} x^{\prime} \\
\quad+\mu \int_{\Omega_{ \pm}} \rho c^{2}\left|\nabla^{\psi} \cdot \partial^{\alpha} u\right|^{2} \mathrm{~d} x \\
=\int_{\Omega_{ \pm}} R^{3}(U, \psi) \partial^{\alpha} p+\left(R^{3}(U, \psi)+\mu R^{3}(U, \psi, \dot{p})\right) \cdot \partial^{\alpha} u \\
\quad+\mu R^{3}(U, \psi) \nabla^{\psi} \cdot \partial^{\alpha} u \mathrm{~d} x .
\end{aligned}
$$

We integrate in time from 0 to $t$, apply Cauchy's inequality and use the estimates (118) and (124) for the terms on the right hand side. Hence we obtain

$$
\int_{\Omega_{ \pm}} \frac{1}{\rho c^{2}}\left|\partial^{\alpha} p\right|^{2} \mathrm{~d} x+\int_{\Omega_{ \pm}} \rho_{\mu}\left|\partial^{\alpha} u\right|^{2} \mathrm{~d} x+\mu \int_{0}^{t} \int_{\Omega_{ \pm}} \rho c^{2}\left|\nabla^{\psi} \cdot \partial^{\alpha} u\right|^{2} \mathrm{~d} x \mathrm{~d} \tau
$$




$$
\mp 2 \int_{0}^{t} \int_{\Gamma}\left(\partial^{\alpha} p+\mu \partial^{\alpha} \dot{p}\right) \partial^{\alpha} u \cdot n \mathrm{~d} x^{\prime} \mathrm{d} \tau \leqq\left(M^{0}+t K(t)\right)\left(\frac{1}{\varepsilon}+\varepsilon E_{\mu}(t)\right) \text {. }
$$

To deal with the boundary integral over $\Gamma$, which is a key point in the estimates and is where we need to use the presence of surface tension, we must sum over \pm to obtain a jump condition. Doing this we obtain

$$
\begin{aligned}
& \sum_{ \pm} \int_{\Omega_{ \pm}} \frac{1}{\rho c^{2}}\left|\partial^{\alpha} p\right|^{2} \mathrm{~d} x+\sum_{ \pm} \int_{\Omega_{ \pm}} \rho_{\mu}\left|\partial^{\alpha} u\right|^{2} \mathrm{~d} x \\
& \quad+\mu \sum_{ \pm} \int_{0}^{t} \int_{\Omega_{ \pm}} \rho c^{2}\left|\nabla^{\psi} \cdot \partial^{\alpha} u\right|^{2} \mathrm{~d} x \mathrm{~d} \tau \\
& -2 I^{\alpha} \leqq\left(M^{0}+t K(t)\right)\left(\frac{1}{\varepsilon}+\varepsilon E_{\mu}(t)\right),
\end{aligned}
$$

where

$$
I^{\alpha}=\int_{0}^{t} \int_{\Gamma}\left[n \cdot \partial^{\alpha} u\left(\partial^{\alpha} p+\mu \partial^{\alpha} \dot{p}\right)\right] \mathrm{d} x^{\prime} \mathrm{d} \tau .
$$

To estimate $I^{\alpha}$, firstly, we use the differentiated equation for the front, (79), to replace $n \cdot \partial^{\alpha} u$, and we obtain the following:

$$
\begin{aligned}
I^{\alpha}= & \int_{0}^{t} \int_{\Gamma}\left[n \cdot \partial^{\alpha} u \partial^{\alpha}(p+\mu \dot{p})\right] \mathrm{d} x^{\prime} \mathrm{d} \tau \\
= & \int_{0}^{t} \int_{\Gamma} \partial_{t} \partial^{\alpha} f\left[\partial^{\alpha}(p+\mu \dot{p})\right] \mathrm{d} x^{\prime} \mathrm{d} \tau+\int_{0}^{t} \int_{\Gamma}\left[u^{\prime} \cdot \partial^{\alpha} \nabla^{\prime} f \partial^{\alpha}(p+\mu \dot{p})\right] \mathrm{d} x^{\prime} \mathrm{d} \tau \\
& +\int_{0}^{t} \int_{\Gamma}\left[R_{\Gamma}^{2}(U, f) \partial^{\alpha}(p+\mu \dot{p})\right] \mathrm{d} x^{\prime} \mathrm{d} \tau \\
= & I_{1}^{\alpha}+I_{2}^{\alpha}+I_{3}^{\alpha},
\end{aligned}
$$

where, as before, $R_{\Gamma}^{2}(U, f)$ denotes a remainder term given in Definition 30 and we feel free to absorb lower order terms of the correct form into this remainder.

Firstly, we note that, using the remainder estimate (125), we have

$$
\left|I_{3}^{\alpha}\right| \leqq \frac{1}{\varepsilon} M^{0}+\frac{1}{\varepsilon} t K(t)+\varepsilon\left(M^{0}+t K(t)\right) E_{\mu}(t) .
$$

Let us consider $I_{1}^{\alpha}$. Using the differentiated pressure interface condition (80), we have

$$
\begin{aligned}
I_{1}^{\alpha}= & -\sigma \int_{0}^{t} \int_{\Gamma} \partial_{t} \partial^{\alpha} f \partial^{\alpha} \nabla^{\prime} \cdot \hat{n} \mathrm{~d} x^{\prime} \mathrm{d} \tau+\mu \int_{0}^{t} \int_{\Gamma} \partial_{t} \partial^{\alpha} f \partial^{\alpha}\left(\Delta^{\prime}-1\right)(u \cdot n) \mathrm{d} x^{\prime} \mathrm{d} \tau \\
& +\int_{0}^{t} \int_{\Gamma} \partial_{t} \partial^{\alpha} f \partial^{\alpha} g_{2} \mathrm{~d} x^{\prime} \mathrm{d} \tau \\
= & \sigma \int_{0}^{t} \int_{\Gamma} \partial_{t} \partial^{\alpha} f \nabla^{\prime} \cdot \partial^{\alpha}\left(\frac{\nabla^{\prime} f}{|n|}\right) \mathrm{d} x^{\prime} \mathrm{d} \tau+\mu \int_{0}^{t} \int_{\Gamma} \partial_{t} \partial^{\alpha} f \partial^{\alpha}\left(\Delta^{\prime}-1\right) \partial_{t} f \mathrm{~d} x^{\prime} \mathrm{d} \tau \\
& +\int_{0}^{t} \int_{\Gamma} R_{\Gamma}^{3}(f)^{2} \mathrm{~d} x^{\prime} \mathrm{d} \tau
\end{aligned}
$$




$$
\begin{aligned}
= & -\sigma \int_{0}^{t} \int_{\Gamma} \partial_{t} \partial^{\alpha} \nabla^{\prime} f \cdot \partial^{\alpha}\left(\frac{\nabla^{\prime} f}{|n|}\right) \mathrm{d} x^{\prime} \mathrm{d} \tau-\mu \int_{0}^{t} \int_{\Gamma}\left|\nabla^{\prime} \partial^{\alpha} \partial_{t} f\right|^{2} \mathrm{~d} x^{\prime} \mathrm{d} \tau \\
& -\mu \int_{0}^{t} \int_{\Gamma}\left|\partial^{\alpha} \partial_{t} f\right|^{2} \mathrm{~d} x^{\prime} \mathrm{d} \tau+\int_{0}^{t} \int_{\Gamma} R_{\Gamma}^{3}(f)^{2} \mathrm{~d} x^{\prime} \mathrm{d} \tau \\
= & I_{11}^{\alpha}-\mu \int_{0}^{t} \int_{\Gamma}\left|\nabla^{\prime} \partial^{\alpha} \partial_{t} f\right|^{2} \mathrm{~d} x^{\prime} \mathrm{d} \tau-\mu \int_{0}^{t} \int_{\Gamma}\left|\partial^{\alpha} \partial_{t} f\right|^{2} \mathrm{~d} x^{\prime} \mathrm{d} \tau \\
& +\int_{0}^{t} \int_{\Gamma} R_{\Gamma}^{3}(f)^{2} \mathrm{~d} x^{\prime} \mathrm{d} \tau,
\end{aligned}
$$

where we have used integration by parts in the second to last line. Assuming there was no factor of $\frac{1}{|n|}$ in $I_{11}^{\alpha}$, we could use the product rule to convert the integrand to $\frac{1}{2} \partial_{t}\left|\partial^{\alpha} \nabla^{\prime} f\right|^{2}$. We intend to do almost the same thing, but to deal with the factor of $\frac{1}{|n|}$ we will need to use Lemma 6.

We have

$$
\begin{aligned}
-I_{11}^{\alpha}= & \sigma \int_{0}^{t} \int_{\Gamma} \partial_{t} \partial^{\alpha} \nabla^{\prime} f \cdot\left(\frac{\partial^{\alpha} \nabla^{\prime} f}{|n|}-\frac{\left(\nabla^{\prime} f \cdot \partial^{\alpha} \nabla^{\prime} f\right) \nabla^{\prime} f}{|n|^{3}}+R_{\Gamma}^{2}(f)\right) \mathrm{d} x^{\prime} \mathrm{d} \tau \\
= & \sigma \int_{0}^{t} \int_{\Gamma} \partial_{t} \partial^{\alpha} \nabla^{\prime} f \cdot \frac{\partial^{\alpha} \nabla^{\prime} f}{|n|}-\partial_{t}\left(\nabla^{\prime} f \cdot \partial^{\alpha} \nabla^{\prime} f\right) \frac{\left(\nabla^{\prime} f \cdot \partial^{\alpha} \nabla^{\prime} f\right)}{|n|^{3}} \mathrm{~d} x^{\prime} \mathrm{d} \tau \\
& +\sigma \int_{0}^{t} \int_{\Gamma} \partial_{t} \partial^{\alpha} \nabla^{\prime} f \cdot R_{\Gamma}^{2}(f) \mathrm{d} x^{\prime} \mathrm{d} \tau+\sigma \int_{0}^{t} \int_{\Gamma} R_{\Gamma}^{3}(f) \cdot R_{\Gamma}^{3}(f) \mathrm{d} x^{\prime} \mathrm{d} \tau \\
= & \frac{1}{2} \sigma \int_{0}^{t} \frac{\mathrm{d}}{\mathrm{d} t} \int_{\Gamma} \frac{1}{|n|}\left|\partial^{\alpha} \nabla^{\prime} f\right|^{2}-\frac{1}{|n|^{3}}\left|\nabla^{\prime} f \cdot \partial^{\alpha} \nabla^{\prime} f\right|^{2} \mathrm{~d} x^{\prime} \mathrm{d} \tau \\
& -\sigma \int_{0}^{t} \int_{\Gamma} \partial_{t} \partial^{\beta} \nabla^{\prime} f \cdot \partial_{x_{i}} R_{\Gamma}^{2}(f) \mathrm{d} x^{\prime} \mathrm{d} \tau+\sigma \int_{0}^{t} \int_{\Gamma} R_{\Gamma}^{3}(f) \cdot R_{\Gamma}^{3}(f) \mathrm{d} x^{\prime} \mathrm{d} \tau \\
\geqq & \frac{1}{2} \sigma \int_{\Gamma} \frac{1}{|n|}\left|\partial^{\alpha} \nabla^{\prime} f\right|^{2}-\frac{1}{|n|^{3}}\left|\nabla^{\prime} f \cdot \partial^{\alpha} \nabla^{\prime} f\right|^{2} \mathrm{~d} x^{\prime}-M^{0} \\
& -\sigma \int_{0}^{t}\left\|\partial_{t} \partial^{\beta} f\right\|_{H^{1}(\Gamma)}\left\|R_{\Gamma}^{2}(f)\right\|_{H^{1}(\Gamma)} \mathrm{d} \tau-\sigma \int_{0}^{t}\left\|R_{\Gamma}^{3}(f)\right\|_{L^{2}(\Gamma)}^{2} \mathrm{~d} \tau,
\end{aligned}
$$

where we have used the fact that $\partial^{\alpha}=\partial_{x_{i}} \partial^{\beta}$ together with integration by parts for the second term on the right. We now apply Lemma 7 to the first term on the right hand side.

Hence

$$
-I_{11}^{\alpha} \geqq \frac{1}{2} \sigma \int_{\Gamma} \frac{1}{|n|^{3}}\left|\partial^{\alpha} \nabla^{\prime} f\right|^{2} \mathrm{~d} x^{\prime}-M^{0}-t K(t),
$$

where we have used the remainder estimate (120).

Hence we obtain

$$
\begin{aligned}
-I_{1}^{\alpha} \geqq & \frac{1}{2} \sigma \int_{\Gamma} \frac{1}{|n|^{3}}\left|\partial^{\alpha} \nabla^{\prime} f\right|^{2} \mathrm{~d} x^{\prime}+\mu \int_{0}^{t} \int_{\Gamma}\left|\nabla^{\prime} \partial^{\alpha}(u \cdot n)\right|^{2} \mathrm{~d} x^{\prime} \mathrm{d} \tau \\
& +\mu \int_{0}^{t} \int_{\Gamma}\left|\partial^{\alpha}(u \cdot n)\right|^{2} \mathrm{~d} x^{\prime} \mathrm{d} \tau-M^{0}-t K(t) .
\end{aligned}
$$


Now we proceed to estimate $I_{2}^{\alpha}$. We have

$$
\begin{aligned}
\left|I_{2}^{\alpha}\right| \leqq & \sum_{ \pm}\left|\int_{0}^{t} \int_{\Gamma} u_{ \pm}^{\prime} \cdot \partial^{\alpha} \nabla^{\prime} f \partial^{\alpha}\left(p_{ \pm}+\mu \dot{p}_{ \pm}\right) \mathrm{d} x^{\prime} \mathrm{d} \tau\right| \\
\leqq & \sum_{ \pm} \int_{0}^{t}\left\|u_{ \pm}^{\prime} \cdot \partial_{x_{i}} \partial^{\beta} \nabla^{\prime} f\right\|_{H^{0.5}(\Gamma)}\left\|\partial_{x_{i}} \partial^{\beta}\left(p_{ \pm}+\mu \dot{p}_{ \pm}\right)\right\|_{H^{-0.5}(\Gamma)} \mathrm{d} \tau \\
\leqq & \sum_{ \pm} \int_{0}^{t}\left\|u_{ \pm}\right\|_{W^{1, \infty}(\Gamma)}^{2}\left\|\partial_{x_{i}} \partial^{\beta} \nabla^{\prime} f\right\|_{H^{0.5}(\Gamma)}^{2} \mathrm{~d} t+\sum_{ \pm} \int_{0}^{t}\left\|\partial^{\beta} p_{ \pm}\right\|_{H^{0.5}(\Gamma)}^{2} \mathrm{~d} \tau \\
& +\mu^{2} \sum_{ \pm} \int_{0}^{t}\left\|\partial^{\beta} \dot{p}_{ \pm}\right\|_{H^{0.5}(\Gamma)}^{2} \mathrm{~d} \tau \\
\leqq & t K(t),
\end{aligned}
$$

where we have again used the Sobolev trace estimate and the estimate (111) for $\dot{p}$.

Putting what we have so far together and using the inequalities (56)-(57) and the lower order estimates (62)-(63), we have

$$
\begin{aligned}
& \sum_{ \pm}\left\|\partial^{\alpha} p\right\|_{L^{2}\left(\Omega_{ \pm}\right)}^{2}+\sum_{ \pm}\left\|\partial^{\alpha} u\right\|_{L^{2}\left(\Omega_{ \pm}\right)}^{2}+\mu \sum_{ \pm} \int_{0}^{t}\left\|\nabla^{\psi} \cdot \partial^{\alpha} u\right\|_{L^{2}\left(\Omega_{ \pm}\right)}^{2} \mathrm{~d} \tau \\
& \quad+\left\|\partial^{\alpha} \nabla^{\prime} f\right\|_{L^{2}(\Gamma)}^{2}+\mu \int_{0}^{t}\left\|\partial^{\alpha}(u \cdot n)\right\|_{H^{1}(\Gamma)}^{2} \mathrm{~d} \tau \\
& \quad \leqq\left(M^{0}+t K(t)\right)\left(\frac{1}{\varepsilon}+\varepsilon E_{\mu}(t)\right) .
\end{aligned}
$$

9.10. Estimate of the Time Derivatives of the Corrected Pressure and Velocity and the Front

We cannot apply the elliptic-type estimate for the front to estimate the highest order time derivatives, although part of the approach will be similar to the previous section. Instead we will need to use the structure of the equations. We also need to use the corrected unknowns in order to deal with the highest order time derivative of $\psi$.

We let $\beta$ be a multi-index with $|\beta|=2, \beta_{3}=0$ and $\beta_{0} \geqq 1$, then we apply the operator $D_{t}^{u_{-}}:=\partial_{t}+u_{-}^{\psi} \cdot \nabla$ (with $u_{-}$defined on $\Omega_{+}$by Sobolev extension) to the corrected pressure and velocity equations (76)-(77) with index $\beta$ to obtain the following equations:

$$
\begin{aligned}
& \frac{1}{\rho c^{2}}\left(\partial_{t}+u^{\psi} \cdot \nabla\right) D_{t}^{u_{-}} p^{\beta}+\nabla^{\psi} D_{t}^{u_{-}} u^{\beta}=R^{3}(U, \psi) \\
& \rho_{\mu}\left(\partial_{t}+u^{\psi} \cdot \nabla\right) D_{t}^{u_{-}} u^{\beta}+\nabla^{\psi} D_{t}^{u_{-}} p^{\beta}+\mu \nabla^{\psi} D_{t}^{u_{-}} \partial^{\beta} \dot{p}=R^{3}(U, \psi) \\
& \quad+\mu R^{3}(U, \psi, \dot{p}) .
\end{aligned}
$$


We now proceed as for the tangential derivatives. Multiplying (84) by $D_{t}^{u_{-}} p^{\beta}$ and (85) by $D_{t}^{u_{-}} u^{\beta}$, integrating over $\Omega_{ \pm}$and summing over \pm and integrating from 0 to $t$, we obtain

$$
\begin{aligned}
& \sum_{ \pm} \int_{\Omega_{ \pm}} \frac{1}{\rho c^{2}}\left|D_{t}^{u_{-}} p^{\beta}\right|^{2} \mathrm{~d} x+\sum_{ \pm} \int_{\Omega_{ \pm}} \rho_{\mu}\left|D_{t}^{u_{-}} u^{\beta}\right|^{2} \mathrm{~d} x \\
& \quad+\mu \sum_{ \pm} \int_{0}^{t} \int_{\Omega_{ \pm}} \rho c^{2}\left|\nabla^{\psi} \cdot\left(D_{t}^{u_{-}} \partial^{\beta} u\right)\right|^{2} \mathrm{~d} x \mathrm{~d} \tau \\
& \quad-2 \int_{0}^{t} \int_{\Gamma}\left[n \cdot D_{t}^{u_{-}} u^{\beta}\left(D_{t}^{u_{-}} p^{\beta}+\mu D_{t}^{u_{-}} \partial^{\beta} \dot{p}\right)\right] \mathrm{d} x^{\prime} \mathrm{d} \tau \\
& \leqq \\
& \quad\left(M^{0}+t K(t)\right)\left(\frac{1}{\varepsilon}+\varepsilon E_{\mu}(t)\right),
\end{aligned}
$$

where we have used the remainder estimates (118) and (124).

Define

$$
K^{\beta}=\int_{0}^{t} \int_{\Gamma}\left[n \cdot D_{t}^{u_{-}} u^{\beta}\left(D_{t}^{u_{-}} p^{\beta}+\mu D_{t}^{u_{-}} \partial^{\beta} \dot{p}\right)\right] \mathrm{d} x^{\prime} \mathrm{d} \tau
$$

First we go back to using the original rather than the corrected unknowns by replacing $u^{\beta}$ with $\partial^{\beta} u-\frac{\partial^{\beta} \psi}{J \psi} \partial_{x_{3}} u$ and replacing $p^{\beta}$ similarly to obtain

$$
K^{\beta}=\int_{0}^{t} \int_{\Gamma}\left[n \cdot D_{t}^{u_{-}} u^{\beta}\left(D_{t}^{u_{-}} p^{\beta}+\mu D_{t}^{u_{-}} \partial^{\beta} \dot{p}\right)\right] \mathrm{d} x^{\prime} \mathrm{d} \tau=K_{1}^{\beta}-K_{2}^{\beta}-K_{3}^{\beta}+K_{4}^{\beta}
$$

where

$$
K_{1}^{\beta}=\int_{0}^{t} \int_{\Gamma}\left[n \cdot D_{t}^{u_{-}} \partial^{\beta} u D_{t}^{u_{-}} \partial^{\beta}(p+\mu \dot{p})\right] \mathrm{d} x^{\prime} \mathrm{d} \tau
$$

and $K_{2}^{\beta}, K_{3}^{\beta}, K_{4}^{\beta}$ are the other terms generated when expanding the brackets. Using the remainder estimates (119) and (125) it is almost immediate that

$$
\left|K_{2}^{\beta}\right|+\left|K_{3}^{\beta}\right|+\left|K_{4}^{\beta}\right| \leqq\left(M^{0}+t K(t)\right)\left(\frac{1}{\varepsilon}+\varepsilon E_{\mu}(t)\right) .
$$

We now consider $K_{1}^{\beta}$. We use the differentiated equation for the front, (79), to replace $n \cdot D_{t}^{u_{-}} \partial^{\beta} u$, and we obtain the following:

$$
\begin{aligned}
K_{1}^{\beta}= & \int_{0}^{t} \int_{\Gamma}\left[n \cdot D_{t}^{u_{-}} \partial^{\beta} u D_{t}^{u_{-}} \partial^{\beta}(p+\mu \dot{p})\right] \mathrm{d} x^{\prime} \mathrm{d} \tau \\
= & \int_{0}^{t} \int_{\Gamma} \partial_{t} D_{t}^{u_{-}} \partial^{\beta} f\left[D_{t}^{u_{-}} \partial^{\beta}(p+\mu \dot{p})\right] \mathrm{d} x^{\prime} \mathrm{d} \tau \\
& +\int_{0}^{t} \int_{\Gamma}\left[u^{\prime} \cdot D_{t}^{u_{-}} \partial^{\beta} \nabla^{\prime} f D_{t}^{u_{-}} \partial^{\beta}(p+\mu \dot{p})\right] \mathrm{d} x^{\prime} \mathrm{d} \tau
\end{aligned}
$$




$$
\begin{aligned}
& +\int_{0}^{t} \int_{\Gamma}\left[R_{\Gamma}^{2}(U, f) D_{t}^{u_{-}} \partial^{\beta}(p+\mu \dot{p})\right] \mathrm{d} x^{\prime} \mathrm{d} \tau \\
= & \int_{0}^{t} \int_{\Gamma} \partial_{t} D_{t}^{u_{-}} \partial^{\beta} f\left[D_{t}^{u_{-}} \partial^{\beta}(p+\mu \dot{p})\right] \mathrm{d} x^{\prime} \mathrm{d} \tau \\
& +\int_{0}^{t} \int_{\Gamma}\left(u_{+}^{\prime} \cdot \nabla^{\prime}\right) D_{t}^{u_{-}} \partial^{\beta} f\left[D_{t}^{u_{-}} \partial^{\beta}(p+\mu \dot{p})\right] \mathrm{d} x^{\prime} \mathrm{d} \tau \\
& +\int_{0}^{t} \int_{\Gamma}\left(\left[u^{\prime}\right] \cdot \nabla^{\prime}\right) D_{t}^{u_{-}} \partial^{\beta} f D_{t}^{u_{-}} \partial^{\beta}\left(p_{-}+\mu \dot{p}_{-}\right) \mathrm{d} x^{\prime} \mathrm{d} \tau \\
& +\int_{0}^{t} \int_{\Gamma}\left[R_{\Gamma}^{2}(U, f) D_{t}^{u_{-}} \partial^{\beta}(p+\mu \dot{p})\right] \mathrm{d} x^{\prime} \mathrm{d} \tau \\
= & K_{11}^{\beta}+K_{12}^{\beta}+K_{13}^{\beta}+K_{14}^{\beta} .
\end{aligned}
$$

Applying the remainder estimate lemma (125), we have

$$
\left|K_{14}^{\beta}\right| \leqq\left(M^{0}+t K(t)\right)\left(\frac{1}{\varepsilon}+\varepsilon E_{\mu}(t)\right) .
$$

The estimate of $K_{11}^{\beta}$ is similar to the estimate of $I_{1}^{\alpha}$. Using the differentiated pressure interface condition (80) with $\partial^{\alpha}$ replaced by $D_{t}^{u_{-}} \partial^{\beta}$ and integrating by parts, we have

$$
\begin{aligned}
K_{11}^{\beta}= & -\sigma \int_{0}^{t} \int_{\Gamma} \partial_{t} D_{t}^{u_{-}} \partial^{\beta} f D_{t}^{u_{-}} \partial^{\beta} \nabla^{\prime} \cdot \hat{n}+\mu \partial_{t} D_{t}^{u_{-}} \partial^{\beta} f D_{t}^{u_{-}} \partial^{\beta}\left(\Delta^{\prime}-1\right) \partial_{t} f \mathrm{~d} x^{\prime} \mathrm{d} \tau \\
& +K_{112}^{\beta} \\
= & \sigma \int_{0}^{t} \int_{\Gamma} \partial_{t} D_{t}^{u_{-}} \partial^{\beta} f \nabla^{\prime} \cdot D_{t}^{u_{-}} \partial^{\beta}\left(\frac{\nabla^{\prime} f}{|n|}\right)+\mu \partial_{t} D_{t}^{u_{-}} \partial^{\beta} f \nabla^{\prime} \cdot D_{t}^{u_{-}} \partial^{\beta} \nabla^{\prime} \partial_{t} f \mathrm{~d} x^{\prime} \mathrm{d} \tau \\
& -\mu \int_{0}^{t} \int_{\Gamma} \partial_{t} D_{t}^{u_{-}} \partial^{\beta} f D_{t}^{u_{-}} \partial^{\beta} \partial_{t} f \mathrm{~d} x^{\prime} \mathrm{d} \tau+K_{112}^{\beta}+K_{113}^{\beta}+K_{114}^{\beta} \\
= & -\sigma \int_{0}^{t} \int_{\Gamma} \partial_{t} D_{t}^{u_{-}} \partial^{\beta} \nabla^{\prime} f \cdot D_{t}^{u_{-}} \partial^{\beta}\left(\frac{\nabla^{\prime} f}{|n|}\right) \\
& -\mu \nabla^{\prime} \partial_{t} D_{t}^{u_{-}} \partial^{\beta} f \cdot D_{t}^{u_{-}} \partial^{\beta} \nabla^{\prime} \partial_{t} f \mathrm{~d} x^{\prime} \mathrm{d} \tau \\
& -\mu \int_{0}^{t} \int_{\Gamma} \partial_{t} D_{t}^{u_{-}} \partial^{\beta} f D_{t}^{u_{-}} \partial^{\beta} \partial_{t} f \mathrm{~d} x^{\prime} \mathrm{d} \tau+K_{112}^{\beta}+K_{113}^{\beta}+K_{114}^{\beta}+K_{115}^{\beta} \\
= & -\sigma \int_{0}^{t} \int_{\Gamma} \partial_{t} D_{t}^{u_{-}} \partial^{\beta} \nabla^{\prime} f \cdot D_{t}^{u_{-}} \partial^{\beta}\left(\frac{\nabla^{\prime} f}{|n|}\right) \mathrm{d} x^{\prime} \mathrm{d} \tau \\
& -\mu \int_{0}^{t} \int_{\Gamma}\left|\nabla^{\prime} D_{t}^{u_{-}} \partial^{\beta} \partial_{t} f\right|^{2}+\left|D_{t}^{u_{-}} \partial^{\beta} \partial_{t} f\right|^{2} \mathrm{~d} x^{\prime} \mathrm{d} \tau+\sum_{i=2}^{7} K_{11 i}^{\beta},
\end{aligned}
$$

where

$$
K_{112}^{\beta}=\int_{0}^{t} \int_{\Gamma} \partial_{t} D_{t}^{u_{-}} \partial^{\beta} f D_{t}^{u_{-}} \partial^{\beta} g_{2} \mathrm{~d} x^{\prime} \mathrm{d} \tau
$$


and each of $K_{113}^{\beta}, \ldots, K_{117}^{\beta}$ denotes a remainder term generated when a derivative hits the $u_{-}^{\prime}$ in $D_{t}^{u_{-}}$and we will omit to write them out explicitly. Let us also label the first term on the right as

$$
K_{111}^{\beta}=-\sigma \int_{0}^{t} \int_{\Gamma} \partial_{t} D_{t}^{u_{-}} \partial^{\beta} \nabla^{\prime} f \cdot D_{t}^{u_{-}} \partial^{\beta}\left(\frac{\nabla^{\prime} f}{|n|}\right) \mathrm{d} x^{\prime} \mathrm{d} \tau .
$$

The remainder terms $K_{112}^{\beta}, \ldots, K_{117}^{\beta}$ can be estimated using Holder's inequality together with the Sobolev embedding theorem, the Sobolev trace estimate, the chain rule, the lower order estimates (62)-(65), the existing tangential estimate for $\nabla^{\prime} f$ given in (83) and integration by parts in space and time. We omit these technical estimates, which are made slightly more involved than one would like by the fact that we are working with a relatively low order energy functional (ie it contains 3 rather than 4 derivatives of $u$ ). We merely state that

$$
\sum_{i=2}^{7}\left|K_{11 i}^{\beta}\right| \leqq\left(M^{0}+t K(t)\right)\left(\frac{1}{\varepsilon}+\varepsilon E_{\mu}(t)\right)
$$

Having estimated the lower order terms, we return to the more important estimate of $K_{111}^{\beta}$. We use Lemma 6 to obtain the following:

$$
\begin{aligned}
-K_{111}^{\beta}= & \sigma \int_{0}^{t} \int_{\Gamma} \partial_{t} D_{t}^{u_{-}} \partial^{\beta} \nabla^{\prime} f \cdot\left(\frac{D_{t}^{u_{-}} \partial^{\beta} \nabla^{\prime} f}{|n|}-\frac{\left(\nabla^{\prime} f \cdot D_{t}^{u_{-}} \partial^{\beta} \nabla^{\prime} f\right) \nabla^{\prime} f}{|n|^{3}}\right) \mathrm{d} x^{\prime} \mathrm{d} \tau \\
& +\int_{0}^{t} \int_{\Gamma} \partial_{t} D_{t}^{u_{-}} \partial^{\beta} \nabla^{\prime} f \cdot\left(R_{\infty, \Gamma}^{0}(U, f) R_{\Gamma}^{2}(f)\right) \mathrm{d} x^{\prime} \mathrm{d} \tau \\
= & \sigma \int_{0}^{t} \int_{\Gamma} \partial_{t} D_{t}^{u_{-}} \partial^{\beta} \nabla^{\prime} f \cdot \frac{D_{t}^{u_{-}} \partial^{\beta} \nabla^{\prime} f}{|n|} \\
& -\partial_{t}\left(\nabla^{\prime} f \cdot D_{t}^{u_{-}} \partial^{\beta} \nabla^{\prime} f\right) \frac{\left(\nabla^{\prime} f \cdot D_{t}^{u_{-}} \partial^{\beta} \nabla^{\prime} f\right)}{|n|^{3}} \mathrm{~d} x^{\prime} \mathrm{d} \tau \\
& +\int_{0}^{t} \int_{\Gamma} \partial_{t} D_{t}^{u_{-}} \partial^{\beta} \nabla^{\prime} f \cdot R_{\infty, \Gamma}^{0}(U, f) R_{\Gamma}^{2}(f) \mathrm{d} x^{\prime} \mathrm{d} \tau \\
& +\int_{0}^{t} \int_{\Gamma} R_{\infty, \Gamma}^{0}(U, f) R_{\Gamma}^{3}(f) R_{\Gamma}^{3}(f) \mathrm{d} x^{\prime} \mathrm{d} \tau \\
= & \frac{1}{2} \int_{0}^{t} \sigma \frac{\mathrm{d}}{\mathrm{d} t} \int_{\Gamma} \frac{1}{|n|}\left|D_{t}^{u_{-}} \partial^{\beta} \nabla^{\prime} f\right|^{2}-\frac{1}{|n|^{3}}\left|\nabla^{\prime} f \cdot D_{t}^{u} \partial^{\beta} \nabla^{\prime} f\right|^{2} \mathrm{~d} x^{\prime} \mathrm{d} \tau \\
& +\int_{0}^{t} \int_{\Gamma} \partial_{t} D_{t}^{u_{-}} \partial^{\beta} \nabla^{\prime} f \cdot R_{\infty, \Gamma}^{0}(U, f) R_{\Gamma}^{2}(f) \mathrm{d} x^{\prime} \mathrm{d} \tau \\
& +\int_{0}^{t} \int_{\Gamma} R_{\infty, \Gamma}^{0}(U, f) R_{\Gamma}^{3}(f) R_{\Gamma}^{3}(f) \mathrm{d} x^{\prime} \mathrm{d} \tau .
\end{aligned}
$$

We now apply Lemma 7 to the first term on the right hand side and we apply the remainder estimates (114) and (120) to the last term. The other term is estimated using integration by parts in time, followed by Cauchy's inequality and the remainder estimates (114), (117), (120) and (123). 
We obtain

$$
-K_{111}^{\beta} \geqq \frac{1}{2} \sigma \int_{\Gamma} \frac{1}{|n|^{3}}\left|D_{t}^{u_{-}} \partial^{\beta} \nabla^{\prime} f\right|^{2} \mathrm{~d} x^{\prime}-\left(M^{0}+t K(t)\right)\left(\frac{1}{\varepsilon}+\varepsilon E_{\mu}(t)\right) .
$$

Thus we have shown that

$$
-K_{11}^{\beta} \geqq \frac{1}{2} \sigma \int_{\Gamma} \frac{1}{|n|^{3}}\left|D_{t}^{u_{-}} \partial^{\beta} \nabla^{\prime} f\right|^{2} \mathrm{~d} x^{\prime}-\left(M^{0}+t K(t)\right)\left(\frac{1}{\varepsilon^{\frac{3}{2}}}+\varepsilon^{\frac{1}{2}} E_{\mu}(t)\right) .
$$

The estimate of $K_{12}^{\beta}$ is similar to the estimate of $K_{11}^{\beta}$, except this time the remainder terms are easier to deal with because we have replaced a time derivative with a tangential derivative. In the end instead of obtaining an exact time derivative, we will obtain an exact horizontal derivative which disappears using the divergence theorem. One complication arises from the fact that the term $-\mu \int_{0}^{t} \int_{\Gamma}\left|\nabla^{\prime} D_{t}^{u_{-}} \partial^{\beta} \partial_{t} f\right|^{2} \mathrm{~d} x^{\prime} \mathrm{d} \tau$ is replaced with the term $\mu \int_{0}^{t} \int_{\Gamma}\left(u_{+}\right.$. $\left.\nabla^{\prime}\right) \nabla^{\prime} D_{t}^{u_{-}} \partial^{\beta} f \cdot \nabla^{\prime} D_{t}^{u_{-}} \partial^{\beta} \partial_{t} f \mathrm{~d} x^{\prime} \mathrm{d} \tau$, which does not have a sign, but we may estimate this using Cauchy's inequality and the existing tangential estimate (83), and the fact that $\partial^{\beta} f=\partial_{t} \partial^{\gamma} f$ for some $|\gamma|=1$ (from the assumption $\beta_{0} \geqq 1$ ) together with the presence of the $\mu$-dependent term involving $\partial_{t} f=u \cdot n$ in the energy. Thus we obtain

$$
\left|K_{12}^{\beta}\right| \leqq\left(M^{0}+t K(t)\right)\left(\frac{1}{\varepsilon^{\frac{3}{2}}}+\varepsilon^{\frac{1}{2}} E_{\mu}(t)\right) .
$$

We proceed to estimate $K_{13}^{\beta}$. Note that, by rearranging the differentiated equation (79) for the front, we obtain

$$
D_{t}^{u_{-}} \partial^{\beta} f=\partial^{\beta} u_{-} \cdot n+R_{\Gamma}^{|\beta|-1}(U, f) .
$$

Using the equation (86), we have

$$
\begin{aligned}
K_{13}^{\beta}= & \int_{0}^{t} \int_{\Gamma} n \cdot\left(\left[u^{\prime}\right] \cdot \nabla^{\prime}\right) \partial^{\beta} u_{-} D_{t}^{u_{-}} \partial^{\beta}\left(p_{-}+\mu \dot{p}_{-}\right) \mathrm{d} x^{\prime} \mathrm{d} \tau \\
& +\int_{0}^{t} \int_{\Gamma} R_{\Gamma}^{2}(U, f) \cdot \partial \partial^{\beta}\left(p_{-}+\mu \dot{p}_{-}\right) \mathrm{d} x^{\prime} \mathrm{d} \tau .
\end{aligned}
$$

We want to convert this into an integral over the interior $\Omega_{-}$. First of all we need to go back to using the corrected pressure and velocity. We obtain

$$
\begin{aligned}
K_{13}^{\beta}= & \int_{0}^{t} \int_{\Gamma} n \cdot\left(\left[u^{\prime}\right] \cdot \nabla^{\prime}\right) u_{-}^{\beta} D_{t}^{u_{-}}\left(p_{-}^{\beta}+\mu \partial^{\beta} \dot{p}_{-}\right) \mathrm{d} x^{\prime} \mathrm{d} \tau \\
& +\int_{0}^{t} \int_{\Gamma} R_{\Gamma}^{2}(U, f) \cdot \partial \partial^{\beta}\left(p_{-}+\mu \dot{p}_{-}\right) \mathrm{d} x^{\prime} \mathrm{d} \tau \\
& +\int_{0}^{t} \int_{\Gamma} \nabla^{\prime} \partial^{\beta} u_{-} \cdot R_{\Gamma}^{2}(U, f) \mathrm{d} x^{\prime} \mathrm{d} \tau+\int_{0}^{t} \int_{\Gamma} R_{\Gamma}^{2}(U, f) \cdot R_{\Gamma}^{2}(U, f) \mathrm{d} x^{\prime} \mathrm{d} \tau \\
= & K_{131}^{\beta}+K_{132}^{\beta}+K_{133}^{\beta}+K_{134}^{\beta} .
\end{aligned}
$$


Using the remainder estimates (119) and (125), we have

$$
\left|K_{132}^{\beta}+K_{133}^{\beta}+K_{134}^{\beta}\right| \leqq\left(M^{0}+t K(t)\right)\left(\frac{1}{\varepsilon}+\varepsilon E_{\mu}(t)\right) .
$$

To estimate $K_{131}^{\beta}$ we convert the boundary integral into an integral over the interior $\Omega_{-}$. Note that we may add the integral of derivatives in the tangential directions because these integrate to zero. We also note that $\left.\left(-\nabla^{\prime} \psi, 1\right)\right|_{\Gamma}=n$, and $\left.J^{\psi}\right|_{\Gamma}=1$. Note that by extending $u_{+}$to $\Omega_{-}$by Sobolev extension we obtain a natural definition for $\left[u^{\prime}\right]=u_{+}-u_{-}$in $\Omega_{-}$. We obtain

$$
\begin{aligned}
K_{131}^{\beta}= & \int_{0}^{t} \int_{\Omega_{-}} \nabla^{\prime} \cdot\left(\left(\left[u^{\prime}\right] \cdot \nabla^{\prime}\right) u^{\beta} D_{t}^{u_{-}}\left(p^{\beta}+\mu \partial^{\beta} \dot{p}\right)\right) \\
& +\partial_{x_{3}}\left(\frac{1}{J^{\psi}}\left(-\nabla^{\prime} \psi, 1\right) \cdot\left(\left[u^{\prime}\right] \cdot \nabla^{\prime}\right) u^{\beta} D_{t}^{u_{-}}\left(p^{\beta}+\mu \partial^{\beta} \dot{p}\right)\right) \mathrm{d} x \mathrm{~d} \tau \\
= & \int_{0}^{t} \int_{\Omega_{-}}\left(\left[u^{\prime}\right] \cdot \nabla^{\prime}\right)\left(\nabla^{\prime} \cdot u^{\beta}+\frac{\left(-\nabla^{\prime} \psi, 1\right)}{J^{\psi}} \cdot \partial_{x_{3}} u^{\beta}\right) D_{t}^{u_{-}}\left(p^{\beta}+\mu \partial^{\beta} \dot{p}\right) \mathrm{d} x \mathrm{~d} \tau \\
& +\int_{0}^{t} \int_{\Omega_{-}}\left(\left[u^{\prime}\right] \cdot \nabla^{\prime}\right) u^{\beta} \cdot D_{t}^{u_{-}}\left(\nabla^{\prime}\left(p^{\beta}+\mu \partial^{\beta} \dot{p}\right)\right. \\
& \left.+\frac{\left(-\nabla^{\prime} \psi, 1\right)}{J^{\psi}} \partial_{x_{3}}\left(p^{\beta}+\mu \partial^{\beta} \dot{p}\right)\right) \mathrm{d} x \mathrm{~d} \tau \\
& +\int_{0}^{t} \int_{\Omega_{-}} R^{3}(U, \psi) \cdot\left(R^{3}(U, \psi)+\mu R^{3}(U, \psi, \dot{p})\right) \mathrm{d} x \mathrm{~d} \tau .
\end{aligned}
$$

Using the corrected pressure and velocity equations (76)-(77), we obtain

$$
\begin{aligned}
K_{131}^{\beta}= & -\int_{0}^{t} \int_{\Omega_{-}} \frac{1}{\rho c^{2}}\left(\left(\left[u^{\prime}\right] \cdot \nabla^{\prime}\right) D_{t}^{u_{-}} p^{\beta} D_{t}^{u_{-}} p^{\beta}\right) \mathrm{d} x \mathrm{~d} \tau \\
& -\int_{0}^{t} \int_{\Omega_{-}} \frac{1}{\rho c^{2}}\left(\left(\left[u^{\prime}\right] \cdot \nabla^{\prime}\right) D_{t}^{u_{-}} p^{\beta} D_{t}^{u_{-}} \mu \partial^{\beta} \dot{p}\right) \mathrm{d} x \mathrm{~d} \tau \\
& -\int_{0}^{t} \int_{\Omega_{-}} \rho_{\mu}\left(\left(\left[u^{\prime}\right] \cdot \nabla^{\prime}\right) u^{\beta} \cdot D_{t}^{u_{-}} D_{t}^{u_{-}} u^{\beta}\right) \mathrm{d} x \mathrm{~d} \tau \\
& +\int_{0}^{t} \int_{\Omega_{-}} R^{3}(U, \psi) \cdot\left(R^{3}(U, \psi)+\mu R^{3}(U, \psi, \dot{p})\right) \mathrm{d} x \mathrm{~d} \tau \\
= & K_{1311}^{\beta}+K_{1312}^{\beta}+K_{1313}^{\beta}+K_{1314}^{\beta}
\end{aligned}
$$
imply

$$
\left|K_{1314}^{\beta}\right| \leqq \frac{1}{\varepsilon} t K(t)+\varepsilon\left(M^{0}+K(t)\right) E_{\mu}(t)
$$

Using the divergence theorem and the remainder estimate (118), we have 


$$
\begin{aligned}
\left|K_{1311}^{\beta}\right| \leqq & \frac{1}{2}\left|\int_{0}^{t} \int_{\Omega_{-}} \nabla^{\prime} \cdot\left(\frac{1}{\rho c^{2}}\left[u^{\prime}\right] D_{t}^{u_{-}} p^{\beta} D_{t}^{u_{-}} p^{\beta}\right) \mathrm{d} x \mathrm{~d} \tau\right| \\
& +\left|\int_{0}^{t} \int_{\Omega_{-}} R^{3}(U, \psi) \cdot R^{3}(U, \psi) \mathrm{d} x \mathrm{~d} \tau\right| \\
\leqq & M^{0}+t K(t) .
\end{aligned}
$$

Using the definition of $\dot{p}=-\rho c^{2} \nabla^{\psi} \cdot u$ and $p^{\beta}$, we have

$$
\begin{aligned}
\left|K_{1312}^{\beta}\right|= & \mu\left|\int_{0}^{t} \int_{\Omega_{-}} \frac{1}{\rho c^{2}}\left(\left[u^{\prime}\right] \cdot \nabla^{\prime}\right) D_{t}^{u_{-}} p^{\beta} D_{t}^{u_{-}} \partial^{\beta} \dot{p} \mathrm{~d} x \mathrm{~d} \tau\right| \\
\leqq & \mu\left|\int_{0}^{t} \int_{\Omega_{-}} \frac{1}{\rho c^{2}}\left(\left[u^{\prime}\right] \cdot \nabla^{\prime}\right) \partial^{\beta} \dot{p} D_{t}^{u_{-}} \partial^{\beta} \dot{p} \mathrm{~d} x \mathrm{~d} \tau\right| \\
& +\mu\left|\int_{0}^{t} \int_{\Omega_{-}} R^{3}(U, \psi) D_{t}^{u} \partial^{\beta} \dot{p} \mathrm{~d} x \mathrm{~d} \tau\right| \\
\leqq & \frac{1}{\tilde{\varepsilon}}\left(M^{0}+t K(t)\right) \mu \int_{0}^{t}\left(\left\|\rho c^{2} \nabla^{\psi} \cdot \nabla^{\prime} \partial^{\beta} u\right\|_{L^{2}\left(\Omega_{-}\right)}^{2}+\left\|R^{3}(U, \psi)\right\|_{L^{2}\left(\Omega_{-}\right)}^{2}\right) \mathrm{d} \tau \\
& +\tilde{\varepsilon} \mu \int_{0}^{t}\left\|\rho c^{2} \nabla^{\psi} \cdot\left(D_{t}^{u} \partial^{\beta} u\right)\right\|_{L^{2}\left(\Omega_{-}\right)}^{2} \mathrm{~d} \tau+\frac{1}{\varepsilon} t K(t)+\varepsilon\left(M^{0}+t K(t)\right) E_{\mu}(t) \\
\leqq & \frac{1}{\tilde{\varepsilon}}\left(M^{0}+t K(t)\right)\left(\frac{1}{\varepsilon}+\varepsilon E_{\mu}(t)\right)+\tilde{\varepsilon} \mu \int_{0}^{t}\left\|\rho c^{2} \nabla^{\psi} \cdot\left(D_{t}^{u_{-}} \partial^{\beta} u\right)\right\|_{L^{2}\left(\Omega_{-}\right)}^{2} \mathrm{~d} \tau,
\end{aligned}
$$

where we have used the lower order estimates (62), (64) and the remainder estimate (124). We have also used crucially the tangential estimate (83) already obtained to estimate the term involving $\mu \int_{0}^{t}\left\|\nabla^{\psi} \cdot \nabla^{\prime} \partial^{\beta} u\right\|_{L^{2}\left(\Omega_{-}\right)}^{2} \mathrm{~d} \tau$.

To estimate $K_{1313}^{\beta}$, we use integration by parts in time and space and the divergence theorem:

$$
\begin{aligned}
\left|K_{1313}^{\beta}\right| \leq & \left|\int_{0}^{t} \int_{\Omega_{-}} D_{t}^{u_{-}}\left(\rho_{\mu}\left(\left[u^{\prime}\right] \cdot \nabla^{\prime}\right) u^{\beta} \cdot D_{t}^{u_{-}} u^{\beta}\right) \mathrm{d} x \mathrm{~d} \tau\right| \\
& +\left|\int_{0}^{t} \int_{\Omega_{-}} \rho_{\mu}\left(\left[u^{\prime}\right] \cdot \nabla^{\prime}\right) D_{t}^{u_{-}} u^{\beta} \cdot D_{t}^{u_{-}} u^{\beta} \mathrm{d} x \mathrm{~d} \tau\right| \\
& +\left|\int_{0}^{t} \int_{\Omega_{-}} R^{3}(U, \psi) \cdot R^{3}(U, \psi) \mathrm{d} x \mathrm{~d} \tau\right| \\
\leqq & \left|\int_{\Omega_{-}} \rho_{\mu}\left(\left[u^{\prime}\right] \cdot \nabla^{\prime}\right) u^{\beta} \cdot D_{t}^{u_{-}} u^{\beta} \mathrm{d} x\right|_{0}^{t} \mid \\
& +\frac{1}{2}\left|\int_{0}^{t} \int_{\Omega_{-}} \nabla \cdot\left(u_{-}^{\psi} \rho_{\mu}\left(\left[u^{\prime}\right] \cdot \nabla^{\prime}\right) u^{\beta} \cdot D_{t}^{u_{-}} u^{\beta}\right) \mathrm{d} x \mathrm{~d} \tau\right| \\
& +\frac{1}{2}\left|\int_{0}^{t} \int_{\Omega_{-}} \nabla^{\prime} \cdot\left(\rho_{\mu}\left[u^{\prime}\right] D_{t}^{u_{-}} u^{\beta} \cdot D_{t}^{u_{-}} u^{\beta}\right) \mathrm{d} x \mathrm{~d} \tau\right|
\end{aligned}
$$




$$
\begin{aligned}
& +\left|\int_{0}^{t} \int_{\Omega_{-}} R^{3}(U, \psi) \cdot R^{3}(U, \psi) \mathrm{d} x \mathrm{~d} \tau\right| \\
\leqq & \left|\int_{\Omega_{-}} R_{\infty}^{0}(U, \psi) \nabla^{\prime} u^{\beta} \cdot \partial u^{\beta} \mathrm{d} x\right|_{0}^{t} \mid+\int_{0}^{t} K(t) \mathrm{d} \tau,
\end{aligned}
$$

where we have used the remainder estimate (118) and the fact that $\left.\left(u_{-}^{\psi}\right)_{3}\right|_{\Gamma}=0$. We now apply Cauchy's lemma to the first term on the right, followed by the estimate we already have for the tangential derivative $\nabla^{\prime} u^{\beta}$ given by (83) together with (75) and the lower order remainder estimate (116), to obtain

$$
\begin{aligned}
\left|K_{1313}^{\beta}\right| & \leqq \frac{1}{\varepsilon^{\frac{1}{2}}}\left\|R_{\infty}^{0}(U, \psi)\right\|_{L^{\infty}\left(\Omega_{-}\right)}^{2}\left\|\nabla^{\prime} u^{\beta}\right\|_{L^{2}\left(\Omega_{-}\right)}^{2}+\varepsilon^{\frac{1}{2}}\left\|\partial u^{\beta}\right\|_{L^{2}\left(\Omega_{-}\right)}^{2}+t K(t) \\
& \leqq\left(M^{0}+t K(t)\right)\left(\frac{1}{\varepsilon^{\frac{3}{2}}}+\varepsilon^{\frac{1}{2}} E_{\mu}(t)\right) .
\end{aligned}
$$

Thus we have eventually shown that

$$
\begin{aligned}
-K^{\beta} \geqq & \frac{1}{2} \sigma \int_{\Gamma} \frac{1}{|n|^{3}}\left|D_{t}^{u_{-}} \partial^{\beta} \nabla^{\prime} f\right|^{2} \mathrm{~d} x^{\prime}+\mu \int_{0}^{t} \int_{\Gamma}\left|\nabla^{\prime} D_{t}^{u_{-}} \partial^{\beta}(u \cdot n)\right|^{2} \\
& +\left|D_{t}^{u_{-}} \partial^{\beta}(u \cdot n)\right|^{2} \mathrm{~d} x^{\prime} \mathrm{d} \tau-\left(M^{0}+t K(t)\right)\left(\frac{1}{\varepsilon^{\frac{3}{2}}}+\varepsilon^{\frac{1}{2}} E_{\mu}(t)\right) \\
& -\frac{1}{\tilde{\varepsilon}}\left(M^{0}+t K(t)\right)\left(\frac{1}{\varepsilon}+\varepsilon E_{\mu}(t)\right)-\tilde{\varepsilon} \mu \int_{0}^{t}\left\|\rho c^{2} \nabla^{\psi} \cdot\left(D_{t}^{u_{-}} \partial^{\beta} u\right)\right\|_{L^{2}\left(\Omega_{-}\right)}^{2} \mathrm{~d} \tau .
\end{aligned}
$$

Hence, choosing $\tilde{\varepsilon}$ sufficiently small so that the term involving $\tilde{\varepsilon}$ above may be absorbed into the left hand side of the estimate below, and using the inequalities (56)-(57) and the lower order estimates (62)-(63), we have

$$
\begin{aligned}
& \sum_{ \pm}\left\|D_{t}^{u_{-}} p^{\beta}\right\|_{L^{2}\left(\Omega_{ \pm}\right)}^{2}+\sum_{ \pm}\left\|D_{t}^{u_{-}} u^{\beta}\right\|_{L^{2}\left(\Omega_{ \pm}\right)}^{2} \\
& \quad+\mu \sum_{ \pm} \int_{0}^{t}\left\|\nabla^{\psi} \cdot\left(D_{t}^{u_{-}} \partial^{\beta} u\right)\right\|_{L^{2}\left(\Omega_{ \pm}\right)}^{2} \mathrm{~d} \tau \\
& \quad+\left\|D_{t}^{u_{-}} \partial^{\beta} \nabla^{\prime} f\right\|_{L^{2}(\Gamma)}^{2}+\mu \int_{0}^{t}\left\|D_{t}^{u_{-}} \partial^{\beta}(u \cdot n)\right\|_{H^{1}(\Gamma)}^{2} \mathrm{~d} \tau \\
& \leqq\left(M^{0}+t K(t)\right)\left(\frac{1}{\varepsilon^{\frac{3}{2}}}+\varepsilon^{\frac{1}{2}} E_{\mu}(t)\right) .
\end{aligned}
$$

In particular, we obtain

$$
\begin{aligned}
& \left\|D_{t}^{u_{-}} \partial^{\beta} \nabla^{\prime} f\right\|_{L^{2}(\Gamma)}^{2}+\mu \int_{0}^{t}\left\|D_{t}^{u_{-}} \partial^{\beta}(u \cdot n)\right\|_{H^{1}(\Gamma)}^{2} \mathrm{~d} \tau \\
& \leqq\left(M^{0}+t K(t)\right)\left(\frac{1}{\varepsilon^{\frac{3}{2}}}+\varepsilon^{\frac{1}{2}} E_{\mu}(t)\right) .
\end{aligned}
$$


Combining this with the tangential estimates for the terms $\mu \int_{0}^{t} \| \nabla^{\prime} \partial^{\beta}(u$. $n) \|_{H^{1}(\Gamma)}^{2} \mathrm{~d} \tau$ and $\left\|\nabla^{\prime} \partial^{\beta} \nabla^{\prime} f\right\|_{L^{2}(\Gamma)}^{2}$ and the lower order estimate (62), we obtain

$$
\begin{aligned}
& \left\|\partial^{\prime} \partial^{\beta} \nabla^{\prime} f\right\|_{L^{2}(\Gamma)}^{2}+\mu \int_{0}^{t}\left\|\partial^{\prime} \partial^{\beta}(u \cdot n)\right\|_{H^{1}(\Gamma)}^{2} \mathrm{~d} \tau \\
& \leqq\left(M^{0}+t K(t)\right)\left(\frac{1}{\varepsilon^{\frac{3}{2}}}+\varepsilon^{\frac{1}{2}} E_{\mu}(t)\right) .
\end{aligned}
$$

Note that if we combine this estimate with the estimate (82) for the corrected entropy and the estimate (73), we obtain the estimate for the entropy

$$
\left\|\partial^{\alpha} s\right\|_{L^{2}\left(\Omega_{ \pm}\right)}^{2} \leqq\left(M^{0}+t K(t)\right)\left(\frac{1}{\varepsilon^{\frac{3}{2}}}+\varepsilon^{\frac{1}{2}} E_{\mu}(t)\right)
$$

for $|\alpha|=3$.

Finally, we convert the estimate (87) into an estimate for the non-corrected pressure and velocity using the estimate (74) together with the estimate (88) for the front. We obtain

$$
\begin{gathered}
\sum_{ \pm}\left\|D_{t}^{u-} \partial^{\beta} p\right\|_{L^{2}\left(\Omega_{ \pm}\right)}^{2}+\sum_{ \pm}\left\|D_{t}^{u-} \partial^{\beta} u\right\|_{L^{2}\left(\Omega_{ \pm}\right)}^{2} \\
\quad+\mu \sum_{ \pm} \int_{0}^{t}\left\|\nabla^{\psi} \cdot\left(D_{t}^{u_{-}} \partial^{\beta} u\right)\right\|_{L^{2}\left(\Omega_{ \pm}\right)}^{2} \mathrm{~d} \tau \\
\leqq\left(M^{0}+t K(t)\right)\left(\frac{1}{\varepsilon^{\frac{3}{2}}}+\varepsilon^{\frac{1}{2}} E_{\mu}(t)\right) .
\end{gathered}
$$

\subsection{A Weighted Normal Derivative Estimate}

It is useful to think of the operator $D_{t}^{u_{ \pm}}$as like a time plus a tangential derivative, which is true on the boundary, since $\left.D_{t}^{u \pm}\right|_{\Gamma}=\partial_{t}+\left(u_{ \pm}^{\psi}\right)^{\prime} \cdot \nabla^{\prime}$ because $\left.\left(u^{\psi}\right)_{3 \pm}\right|_{\Gamma}=0$. But in the interior in fact we have

$$
D_{t}^{u}=\partial_{t}+\left(u^{\psi}\right)^{\prime} \cdot \nabla^{\prime}+\left(u^{\psi}\right)_{3} \partial_{x_{3}} .
$$

Hence it will be useful to have an estimate for $\left(u^{\psi}\right)_{3} \partial_{x_{3}} \partial^{\beta} p$ and $\left(u^{\psi}\right)_{3} \partial_{x_{3}} \partial^{\beta} u$ where $|\beta|=2$, and this will be a straightforward energy estimate since $\left.\left(u^{\psi}\right)_{3}\right|_{\Gamma}=0$.

Let $w$ be given by either $w=\operatorname{Ext}_{\Omega_{-}}\left(u^{\psi}\right)_{3+}$ in $\mathbb{R}^{3}$ or $w=\operatorname{Ext}_{\Omega_{+}}\left(u^{\psi}\right)_{3-}$ in $\mathbb{R}^{3}$ or $w_{ \pm}=\left(u^{\psi}\right)_{3 \pm}$ in $\Omega_{ \pm}$.

We let $\beta$ be a multi-index with $|\beta|=2$. We let $\partial^{\alpha}=\partial_{x_{3}} \partial^{\beta}$ in the differentiated Equations (69)-(70). We then multiply by $w$ to obtain the following:

$$
\begin{aligned}
& \frac{1}{\rho c^{2}}\left(\partial_{t}+u^{\psi} \cdot \nabla\right)\left(w \partial^{\alpha} p\right)+\nabla^{\psi} \cdot\left(w \partial^{\alpha} u\right)=R^{|\alpha|}(U, \psi) \\
& \rho\left(\partial_{t}+u^{\psi} \cdot \nabla\right)\left(w \partial^{\alpha} u\right)+\nabla^{\psi}\left(w \partial^{\alpha} p\right)+\mu \nabla^{\psi}\left(w \partial^{\alpha} \dot{p}\right) \\
& \quad=R^{|\alpha|}(U, \psi)+\mu R^{|\alpha|}(U, \psi, \dot{p}) .
\end{aligned}
$$


We multiply the first equation by $w \partial^{\alpha} p$ and the second by $w \partial^{\alpha} u$ then sum the equations, integrate over $\Omega_{ \pm}$and from 0 to $t$ and use the divergence theorem together with the fact that $w=0$ on $\Gamma$ and the symmetry of the equations plus the inequalities (56) and (57) to obtain

$$
\begin{aligned}
& \int_{\Omega_{ \pm}}\left|w \partial^{\alpha} p\right|^{2} \mathrm{~d} x+\int_{\Omega_{ \pm}}\left|w \partial^{\alpha} u\right|^{2} \mathrm{~d} x+\mu \int_{0}^{t} \int_{\Omega_{ \pm}}\left|w \nabla^{\psi} \cdot \partial^{\alpha} u\right|^{2} \mathrm{~d} x \mathrm{~d} \tau \\
& \quad \leqq\left(M^{0}+t K(t)\right)\left(\frac{1}{\varepsilon}+\varepsilon E_{\mu}(t)\right) .
\end{aligned}
$$

Note that during the estimate we replaced $\partial^{\alpha} \dot{p}$ with $-\rho c^{2} \nabla^{\psi} \cdot \partial^{\alpha} u$ up to lower order terms.

Assume now $\beta$ is a multi-index with $|\beta|=2$ and $\beta_{3}=0$. Combining the above estimate with the high-order time derivative estimate (90), and using the fact that

$$
D_{t}^{u_{-}}=\partial_{t}+\left(u_{-}^{\psi}\right)^{\prime} \cdot \nabla^{\prime}+\left(u^{\psi}\right)_{3-} \partial_{x_{3}},
$$

we obtain the following simpler time derivative estimate:

$$
\begin{aligned}
& \sum_{ \pm}\left\|\partial_{t} \partial^{\beta} p\right\|_{L^{2}\left(\Omega_{ \pm}\right)}^{2}+\sum_{ \pm}\left\|\partial_{t} \partial^{\beta} u\right\|_{L^{2}\left(\Omega_{ \pm}\right)}^{2}+\mu \sum_{ \pm} \int_{0}^{t}\left\|\nabla^{\psi} \cdot \partial_{t} \partial^{\beta} u\right\|_{L^{2}\left(\Omega_{ \pm}\right)}^{2} \mathrm{~d} \tau \\
& \quad \leqq\left(M^{0}+t K(t)\right)\left(\frac{1}{\varepsilon^{\frac{3}{2}}}+\varepsilon^{\frac{1}{2}} E_{\mu}(t)\right)
\end{aligned}
$$

for $|\beta|=2$ with $\beta_{3}=0$.

\subsection{Estimate of the Curl}

We know that the curl of the velocity, $\nabla^{\psi} \times u$, satisfies the Equation (54). We apply $\partial^{\beta}$ to this equation for $1 \leqq|\beta| \leqq 2$ and gather the lower order terms on the right hand side, noting that the high order terms in $\psi$ can be treated as remainder terms since they all involve at most three time derivatives. We obtain

$$
\rho_{\mu}\left(\partial_{t}+u^{\psi} \cdot \nabla\right) \nabla^{\psi} \times \partial^{\beta} u=R^{|\beta|+1}(U, \psi)
$$

in $\left(0, T^{\prime}\right) \times \Omega_{ \pm}$.

For $\beta$ a multi-index with $1 \leqq|\beta| \leqq 2$, we define the corrected curl as

$$
\omega^{\beta}=\nabla^{\psi} \times \partial^{\beta} u \text {. }
$$

Thus the corrected curl satisfies the equation

$$
\rho_{\mu}\left(\partial_{t}+u^{\psi} \cdot \nabla\right) \omega^{\beta}=R^{|\beta|+1}(U, \psi)
$$

in $\left(0, T^{\prime}\right) \times \Omega_{ \pm}$.

Now it is straightforward to estimate the corrected curl in the standard manner, since $\left(u^{\psi}\right)_{3 \pm}=0$ on $\Gamma$ so there is no boundary term. We multiply Equation (95) by $\omega^{\beta}$ and use the product rule, then integrate over $\Omega_{ \pm}$and from 0 to $t$ and use the inequality (57) to obtain

$$
\left\|\omega^{\beta}\right\|_{L^{2}\left(\Omega_{ \pm}\right)}^{2} \leqq M^{0}+t K(t) .
$$




\subsection{Estimate of the Normal Derivatives of the Pressure and Velocity}

For $1 \leqq|\beta| \leqq 2$, we define the corrected divergence as

$$
\eta^{\beta}=\nabla^{\psi} \cdot \partial^{\beta} u
$$

We aim to combine our estimate of the corrected curl along with an estimate of the corrected divergence with $\beta_{3}=0$ to obtain an estimate for $\partial_{x_{3}} \partial^{\beta} u$. We also aim to obtain an estimate of the normal derivative of the pressure directly from the equations. We then use an inductive argument to estimate all the normal derivatives. This is detailed below.

To estimate the normal derivative of the pressure, we will need the following lemma, which is used by Coutand et al. [10] and Coutand and Shroller [13], hence we don't repeat its straightforward proof here.

Lemma 8. Let $w \in W^{1,1}(0, T)$ and let $g \in L^{1}(0, T)$. Let $\mu \geqq 0$ and suppose that

$$
w+\mu \frac{\mathrm{d} w}{\mathrm{~d} t} \leqq g
$$

for almost every $t \in(0, T)$. Then, for any $t \in(0, T)$,

$$
\sup _{\tau \in(0, t)} w(\tau) \leqq w(0)+\underset{\tau \in(0, t)}{\operatorname{ess} \sup }|g(\tau)|
$$

We also need the following lemma, which says that the normal derivative of ${ }_{\partial}^{\beta} u$ can be expressed in terms of the corrected divergence, the corrected curl, the tangential derivatives and the derivatives of $\psi$.

Lemma 9. Let $v \in H^{1}\left(\Omega_{ \pm} ; \mathbb{R}^{3}\right)$. Then

$$
\partial_{x_{3}} v_{i}=G_{i}(\nabla \psi) \cdot\left(\partial_{x_{1}} v, \partial_{x_{2}} v, \nabla^{\psi} \cdot v, \nabla^{\psi} \times v\right),
$$

where $G_{i}(\nabla \psi)$ is a smooth vector-valued function for each $i=1,2,3$.

Proof. First we note that we can write $\nabla^{\psi} \cdot v$ as

$$
\nabla^{\psi} \cdot v=\nabla^{\prime} \cdot v^{\prime}-\frac{\nabla^{\prime} \psi}{J^{\psi}} \cdot \partial_{x_{3}} v^{\prime}+\frac{1}{J^{\psi}} \partial_{x_{3}} v_{3} .
$$

Hence, rearranging,

$$
\partial_{x_{3}} v_{3}=J^{\psi}\left(\nabla^{\psi} \cdot v\right)-J^{\psi} \nabla^{\prime} \cdot v^{\prime}+\nabla^{\prime} \psi \cdot \partial_{x_{3}} v^{\prime} .
$$

From the definition of $\nabla^{\psi} \times$, we have

$$
\begin{aligned}
& \left(\nabla^{\psi} \times v\right)_{1}=\partial_{x_{2}} v_{3}-\partial_{x_{3}} v_{2}-\frac{1}{J \psi} \partial_{x_{2}} \psi \partial_{x_{3}} v_{3}+\frac{1}{J^{\psi}} \partial_{x_{3}} \psi \partial_{x_{3}} v_{2} \\
& \left(\nabla^{\psi} \times v\right)_{2}=\partial_{x_{3}} v_{1}-\partial_{x_{1}} v_{3}-\frac{1}{J \psi} \partial_{x_{3}} \psi \partial_{x_{3}} v_{1}+\frac{1}{J^{\psi}} \partial_{x_{1}} \psi \partial_{x_{3}} v_{3}
\end{aligned}
$$

Rearranging, we obtain 


$$
\begin{aligned}
& \partial_{x_{3}} v_{2}=-J^{\psi}\left(\nabla^{\psi} \times v\right)_{1}+J^{\psi} \partial_{x_{2}} v_{3}-\partial_{x_{2}} \psi \partial_{x_{3}} v_{3} \\
& \partial_{x_{3}} v_{1}=J^{\psi}\left(\nabla^{\psi} \times v\right)_{2}+J^{\psi} \partial_{x_{1}} v_{3}-\partial_{x_{1}} \psi \partial_{x_{3}} v_{3} .
\end{aligned}
$$

Using these two identities in the equation for $\partial_{x_{3}} v_{3}$, we obtain

$$
\begin{aligned}
\partial_{x_{3}} v_{3}= & J^{\psi}\left(\nabla^{\psi} \cdot v\right)-J^{\psi} \nabla^{\prime} \cdot v^{\prime}+\partial_{x_{1}} \psi\left(J^{\psi}\left(\nabla^{\psi} \times v\right)_{2}+J^{\psi} \partial_{x_{1}} v_{3}-\partial_{x_{1}} \psi \partial_{x_{3}} v_{3}\right) \\
& +\partial_{x_{2}} \psi\left(-J^{\psi}\left(\nabla^{\psi} \times v\right)_{1}+J^{\psi} \partial_{x_{2}} v_{3}-\partial_{x_{2}} \psi \partial_{x_{3}} v_{3}\right) .
\end{aligned}
$$

Rearranging, we obtain

$$
\begin{aligned}
\partial_{x_{3}} v_{3}\left(1+\left|\nabla^{\prime} \psi\right|^{2}\right)= & J^{\psi}\left(\nabla^{\psi} \cdot v\right)-J^{\psi} \nabla^{\prime} \cdot v^{\prime}+\partial_{x_{1}} \psi\left(J^{\psi}\left(\nabla^{\psi} \times v\right)_{2}+J^{\psi} \partial_{x_{1}} v_{3}\right) \\
& +\partial_{x_{2}} \psi\left(-J^{\psi}\left(\nabla^{\psi} \times v\right)_{1}+J^{\psi} \partial_{x_{2}} v_{3}\right) .
\end{aligned}
$$

Dividing by $1+\left|\nabla^{\prime} \psi\right|^{2}$, we obtain the desired result for $\partial_{x_{3}} v_{3}$. Putting this expression back into the identities for $\partial_{x_{3}} v_{2}$ and $\partial_{x_{3}} v_{1}$, we obtain the result.

Corollary 1. Let $1 \leqq|\beta| \leqq 2$. Then

$$
\begin{aligned}
& \left\|\partial_{x_{3}} \partial^{\beta} u\right\|_{L^{2}\left(\Omega_{ \pm}\right)} \\
& \quad \leqq\left\|R_{\infty}^{0}(U, \psi)\right\|_{L^{\infty}\left(\Omega_{ \pm}\right)}\left(\left\|\nabla^{\prime} \partial^{\beta} u\right\|_{L^{2}\left(\Omega_{ \pm}\right)}+\left\|\eta^{\beta}\right\|_{L^{2}\left(\Omega_{ \pm}\right)}+\left\|\omega^{\beta}\right\|_{L^{2}\left(\Omega_{ \pm}\right)}\right) .
\end{aligned}
$$

Proof. This is immediate from the above lemma applied to $v=\partial^{\beta} u$, where we have replaced $G_{i}(\nabla \psi)$ by the remainder $R_{\infty}^{0}(U, \psi)$.

Now, let $|\beta|=2$. Note that by (66), and the fact that $\eta^{\beta}=\nabla^{\psi} \cdot \partial^{\beta} u$, we have

$$
\eta^{\beta}=-\frac{1}{\rho c^{2}}\left(\partial_{t}+u^{\psi} \cdot \nabla\right) \partial^{\beta} p+R^{|\beta|}(U, \psi)+R_{\infty}^{0}(U, \psi) \cdot \nabla p \partial_{t} \partial^{\beta} \psi,
$$

where we have absorbed all the high-order terms in $\psi$ involving a spatial derivative into the remainder $R^{|\beta|}(U, \psi)$. Note also that $\left\|\partial_{t} \partial^{\beta} \psi\right\|_{H^{1}\left(\Omega_{ \pm}\right)}$can be estimated by the estimates (81) and (88). Thus

$$
\begin{aligned}
\left\|\eta^{\beta}\right\|_{L^{2}\left(\Omega_{ \pm}\right)}^{2} \leqq & \left(M^{0}+t K(t)\right)\left(1+\left\|\partial_{t} \partial^{\beta} p\right\|_{L^{2}\left(\Omega_{ \pm}\right)}^{2}+\left\|\nabla^{\prime} \partial^{\beta} p\right\|_{L^{2}\left(\Omega_{ \pm}\right)}^{2}\right. \\
& \left.+\left\|\left(u^{\psi}\right)_{3} \partial_{x_{3}} \partial^{\beta} p\right\|_{L^{2}\left(\Omega_{ \pm}\right)}^{2}+\|\nabla p\|_{H^{1}\left(\Omega_{ \pm}\right)}^{2}\left\|\partial_{t} \partial^{\beta} \psi\right\|_{H^{1}\left(\Omega_{ \pm}\right)}^{2}\right) \\
\leqq & \left(M^{0}+t K(t)\right)\left(\frac{1}{\varepsilon^{\frac{3}{2}}}+\varepsilon^{\frac{1}{2}} E_{\mu}(t)+\left\|\partial_{t} \partial^{\beta} p\right\|_{L^{2}\left(\Omega_{ \pm}\right)}^{2}\right. \\
& \left.+\left\|\nabla^{\prime} \partial^{\beta} p\right\|_{L^{2}\left(\Omega_{ \pm}\right)}^{2}\right)
\end{aligned}
$$

where we have used the estimates (56), (62), (64) and the lower order remainder estimate (121), together with the weighted normal derivative estimate (91).

Also, from the third component of (67), we have

$$
\begin{gathered}
\partial_{x_{3}} \partial^{\beta}(p+\mu \dot{p})=-J^{\psi} \rho_{\mu}\left(\partial_{t}+u^{\psi} \cdot \nabla\right) \partial^{\beta} u_{3} \\
+R^{|\beta|}(U, \psi)+R_{\infty}^{0}(U, \psi) \cdot \nabla u_{3} \partial_{t} \partial^{\beta} \psi,
\end{gathered}
$$


where again we have absorbed all the high-order terms in $\psi$ involving a spatial derivative into the remainder $R^{|\beta|}(U, \psi)$ and we note also that $\left\|\partial_{t} \partial^{\beta} \psi\right\|_{H^{1}\left(\Omega_{ \pm}\right)}$can be estimated by the estimates (81) and (88). Now we must take some care to deal with the term involving $\mu \dot{p}$. In fact we chose this approximation to the original equations, as originally done by CouTAND et al. [10] and CouTAND and SHKOLLER [13], specifically so that this term can be easily estimated. We multiply by $\partial_{x_{3}} \partial^{\beta} p$ and integrate in space to obtain

$$
\begin{aligned}
& \frac{1}{2} \int_{\Omega_{ \pm}}\left|\partial_{x_{3}} \partial^{\beta} p\right|^{2} \mathrm{~d} x \leqq-\frac{1}{2} \mu \frac{\mathrm{d}}{\mathrm{d} t} \int_{\Omega_{ \pm}}\left|\partial_{x_{3}} \partial^{\beta} p\right|^{2} \mathrm{~d} x+\mu^{2}\left\|\left(\partial_{x_{3}} \partial^{\beta} u^{\psi}\right) \cdot \nabla p\right\|_{L^{2}\left(\Omega_{ \pm}\right)}^{2} \\
& \quad+\mu \int_{\Omega_{ \pm}}\left(\nabla \cdot u^{\psi}\right)\left|\partial_{x_{3}} \partial^{\beta} p\right|^{2} \mathrm{~d} x+\left(M^{0}+t K(t)\right)\left(\frac{1}{\varepsilon^{\frac{3}{2}}}+\varepsilon^{\frac{1}{2}} E_{\mu}(t)\right. \\
& \left.\quad+\left\|D_{t}^{u_{ \pm}} \partial^{\beta} u\right\|_{L^{2}\left(\Omega_{ \pm}\right)}^{2}\right),
\end{aligned}
$$

where we have used the divergence theorem together with the fact that $\left(u^{\psi}\right)_{3 \pm}=0$ on $\Gamma$, the lower order estimate (64), the lower order remainder estimate (121) and Cauchy's inequality.

Using the fundamental theorem of calculus, together with the fact that $\left.\left(u_{-}^{\psi}\right)_{3}\right|_{\Gamma}=0$ and with the divergence theorem to convert a time derivative into a material derivative, we can show

$$
\mu \int_{\Omega_{ \pm}}\left(\nabla \cdot u^{\psi}\right)\left|\partial_{x_{3}} \partial^{\beta} p\right|^{2} \mathrm{~d} x \leqq M^{0}+t K(t),
$$

where we have used the remainder estimate (118) and the estimate (111) for $\dot{p}$. Similarly, we have

$$
\mu^{2}\left\|\left(\partial_{x_{3}} \partial^{\beta} u^{\psi}\right) \cdot \nabla p\right\|_{L^{2}\left(\Omega_{ \pm}\right)}^{2} \leqq M^{0}+t K(t) .
$$

Combining these estimates, we obtain

$$
\begin{aligned}
& \left\|\partial_{x_{3}} \partial^{\beta} p\right\|_{L^{2}\left(\Omega_{ \pm}\right)}^{2}+\mu \frac{\mathrm{d}}{\mathrm{d} t}\left\|\partial_{x_{3}} \partial^{\beta} p\right\|_{L^{2}\left(\Omega_{ \pm}\right)}^{2} \\
& \leqq\left(M^{0}+t K(t)\right)\left(\frac{1}{\varepsilon^{\frac{3}{2}}}+\varepsilon^{\frac{1}{2}} E_{\mu}(t)+\left\|D_{t}^{u_{ \pm}} \partial^{\beta} u\right\|_{L^{2}\left(\Omega_{ \pm}\right)}^{2}\right) \\
& \quad \leqq\left(M^{0}+t K(t)\right)\left(\frac{1}{\varepsilon^{\frac{3}{2}}}+\varepsilon^{\frac{1}{2}} E_{\mu}(t)+\left\|\partial_{t} \partial^{\beta} u\right\|_{L^{2}\left(\Omega_{ \pm}\right)}^{2}+\left\|\nabla^{\prime} \partial^{\beta} u\right\|_{L^{2}\left(\Omega_{ \pm}\right)}^{2}\right),
\end{aligned}
$$

where we have used the lower order estimates (62), (64) and the weighted normal derivative estimate (91). Applying Lemma 8, we obtain

$$
\begin{aligned}
& \left\|\partial_{x_{3}} \partial^{\beta} p\right\|_{L^{2}\left(\Omega_{ \pm}\right)}^{2} \\
& \leqq \\
& \quad\left(M^{0}+t K(t)\right)\left(\frac{1}{\varepsilon^{\frac{3}{2}}}+\varepsilon^{\frac{1}{2}} E_{\mu}(t)+\underset{\tau \in(0, t)}{\operatorname{ess} \sup \left\|\partial_{t} \partial^{\beta} u\right\|_{L^{2}\left(\Omega_{ \pm}\right)}^{2}}\right. \\
& \quad+\underset{\tau \in(0, t)}{\left.\operatorname{ess} \sup \left\|\nabla^{\prime} \partial^{\beta} u\right\|_{L^{2}\left(\Omega_{ \pm}\right)}^{2}\right)}
\end{aligned}
$$


Now we claim that

$$
\begin{aligned}
\left\|\partial_{x_{3}} \partial^{\beta} u\right\|_{L^{2}\left(\Omega_{ \pm}\right)}^{2} \leqq\left(M^{0}+t K(t)\right)\left(\frac{1}{\varepsilon^{\frac{3}{2}}}+\varepsilon^{\frac{1}{2}} E_{\mu}(t)\right) \\
\left\|\partial_{x_{3}} \partial^{\beta} p\right\|_{L^{2}\left(\Omega_{ \pm}\right)}^{2} \leqq\left(M^{0}+t K(t)\right)\left(\frac{1}{\varepsilon^{\frac{3}{2}}}+\varepsilon^{\frac{1}{2}} E_{\mu}(t)\right) .
\end{aligned}
$$

We proceed by induction on $\beta_{3}$. In the case that $\beta_{3}=0$, we note that we may apply (83) and (92) to the right hand side of (98) and (99) to obtain

$$
\begin{aligned}
&\left\|\eta^{\beta}\right\|_{L^{2}\left(\Omega_{ \pm}\right)}^{2} \leqq\left(M^{0}+t K(t)\right)\left(\frac{1}{\varepsilon^{\frac{3}{2}}}+\varepsilon^{\frac{1}{2}} E_{\mu}(t)\right) \\
&\left\|\partial_{x_{3}} \partial^{\beta} p\right\|_{L^{2}\left(\Omega_{ \pm}\right)}^{2} \leqq\left(M^{0}+t K(t)\right)\left(\frac{1}{\varepsilon^{\frac{3}{2}}}+\varepsilon^{\frac{1}{2}} E_{\mu}(t)\right) .
\end{aligned}
$$

Then we apply the estimate from Corollary 1 together with the lower order remainder estimate (121), the estimate (83) for the tangential derivatives, the estimate (96) for the corrected curl and the estimate just obtained for the corrected divergence to conclude that

$$
\left\|\partial_{x_{3}} \partial^{\beta} u\right\|_{L^{2}\left(\Omega_{ \pm}\right)}^{2} \leqq\left(M^{0}+t K(t)\right)\left(\frac{1}{\varepsilon^{\frac{3}{2}}}+\varepsilon^{\frac{1}{2}} E_{\mu}(t)\right),
$$

which proves the result in the case $\beta_{3}=0$.

Now we suppose the result is true for $\beta_{3} \leqq k$ for some $0 \leqq k \leqq|\beta|-1$ and try to show it is true for $\beta_{3}=k+1$. But this is straightforward and is done in the same way. Note that the previous induction step gives us the estimate for

$$
\begin{aligned}
\left\|\partial^{\gamma} u\right\|_{L^{2}\left(\Omega_{ \pm}\right)}^{2} \leqq\left(M^{0}+t K(t)\right)\left(\frac{1}{\varepsilon^{\frac{3}{2}}}+\varepsilon^{\frac{1}{2}} E_{\mu}(t)\right) \\
\left\|\partial^{\gamma} p\right\|_{L^{2}\left(\Omega_{ \pm}\right)}^{2} \leqq\left(M^{0}+t K(t)\right)\left(\frac{1}{\varepsilon^{\frac{3}{2}}}+\varepsilon^{\frac{1}{2}} E_{\mu}(t)\right)
\end{aligned}
$$

where $|\gamma|=3$ and $\gamma_{3} \leqq k+1$. We apply these estimates to the right hand side of (98) and (99) to obtain

$$
\begin{aligned}
&\left\|\eta^{\beta}\right\|_{L^{2}\left(\Omega_{ \pm}\right)}^{2} \leqq\left(M^{0}+t K(t)\right)\left(\frac{1}{\varepsilon^{\frac{3}{2}}}+\varepsilon^{\frac{1}{2}} E_{\mu}(t)\right) \\
&\left\|\partial_{x_{3}} \partial^{\beta} p\right\|_{L^{2}\left(\Omega_{ \pm}\right)}^{2} \leqq\left(M^{0}+t K(t)\right)\left(\frac{1}{\varepsilon^{\frac{3}{2}}}+\varepsilon^{\frac{1}{2}} E_{\mu}(t)\right) .
\end{aligned}
$$

Then we apply the estimate from Corollary 1 together with the lower order remainder estimate (121), the previous induction step for the term $\nabla^{\prime} \partial^{\beta} u$, the estimate (96) for the corrected curl and the estimate just obtained for the corrected divergence to conclude the result for the case $\beta_{3}=k+1$. This proves the claim. 


\subsection{Estimate of the Artificial Viscosity Term}

Let us observe that we may rearrange the differentiated velocity equation (67) to obtain

$$
\begin{aligned}
-\mu \nabla^{\psi} \partial^{\beta} \dot{p}= & \rho_{\mu}\left(\partial_{t}+u^{\psi} \cdot \nabla\right) \partial^{\beta} u \\
& +\nabla^{\psi} \partial^{\beta} p+R^{|\beta|}(U, \psi)+R_{\infty}^{0}(U, \psi) \nabla \cdot u \partial \partial^{\beta} \psi \\
& +\mu R^{|\beta|}(U, \psi, \dot{p}),
\end{aligned}
$$

where $|\beta| \leqq 2$. We use the fact that we have already obtained estimates for everything on the right hand side in $L^{2}\left(\Omega_{ \pm}\right)$to obtain

$$
\mu^{2}\left\|\nabla^{\psi} \partial^{\beta} \dot{p}\right\|_{L^{2}\left(\Omega_{ \pm}\right)}^{2} \leqq\left(M^{0}+t K(t)\right)\left(\frac{1}{\varepsilon^{\frac{3}{2}}}+\varepsilon^{\frac{1}{2}} E_{\mu}(t)\right) .
$$

Using the fact that

$$
\partial_{x_{3}} \partial^{\beta} \dot{p}=J^{\psi}\left(\nabla^{\psi}\right)_{3} \partial^{\beta} \dot{p}
$$

and the lower order estimate for $\nabla \psi$, we easily obtain

$$
\mu^{2}\left\|\nabla \partial^{\beta} \dot{p}\right\|_{L^{2}\left(\Omega_{ \pm}\right)}^{2} \leqq\left(M^{0}+t K(t)\right)\left(\frac{1}{\varepsilon^{\frac{3}{2}}}+\varepsilon^{\frac{1}{2}} E_{\mu}(t)\right) .
$$

Using the fact that $\dot{p}=-\rho c^{2} \nabla^{\psi} \cdot u$ together with the chain rule and existing estimates we also obtain

$$
\mu^{2}\left\|\nabla^{\psi} \cdot \nabla \partial^{\beta} u\right\|_{L^{2}\left(\Omega_{ \pm}\right)}^{2} \leqq\left(M^{0}+t K(t)\right)\left(\frac{1}{\varepsilon^{\frac{3}{2}}}+\varepsilon^{\frac{1}{2}} E_{\mu}(t)\right)
$$

\subsection{Elliptic-Type Estimate for the Front}

In this section we derive an elliptic type estimate for the front using the pressure interface condition (43).

\section{Lemma 10.}

$$
\left\|\partial^{\alpha} \nabla^{\prime} f\right\|_{H^{0.5}(\Gamma)}^{2} \leqq\left(M^{0}+t K(t)\right)\left(\frac{1}{\varepsilon^{\frac{3}{2}}}+\varepsilon^{\frac{1}{2}} E_{\mu}(t)\right)
$$

for $0 \leqq|\alpha| \leqq 3, \alpha_{0} \leqq 2$ and $\alpha_{3}=0$.

Proof. Let $\alpha$ be a multi-index with $0 \leqq|\alpha| \leqq 3, \alpha_{0} \leqq 2$ and $\alpha_{3}=0$, so that we can write $\partial^{\alpha}=\partial^{\beta} \partial_{x_{i}}$ for some $i \in\{1,2\}$. Let $\left\langle D^{\prime}\right\rangle^{0.5}$ denote the half-order derivative operator which corresponds to multiplying by $\left\langle\xi^{\prime}\right\rangle^{0.5}$ in Fourier space see the notation in the appendix.

Applying $\left\langle D^{\prime}\right\rangle^{0.5}$ to the differentiated pressure interface condition (80), we have 


$$
\begin{aligned}
\left\langle D^{\prime}\right\rangle^{0.5}\left[\partial^{\alpha}(p+\mu \dot{p})\right]= & \sigma\left\langle D^{\prime}\right\rangle^{0.5} \nabla^{\prime} \cdot \partial^{\alpha}\left(\frac{\nabla^{\prime} f}{|n|}\right)+\mu\left(\Delta^{\prime}-1\right)\left\langle D^{\prime}\right\rangle^{0.5} \partial^{\alpha} \partial_{t} f \\
& +\left\langle D^{\prime}\right\rangle^{0.5} \partial^{\alpha} g_{2} .
\end{aligned}
$$

We multiply both sides by $\left\langle D^{\prime}\right\rangle^{0.5} \partial^{\alpha} f$ and integrate by parts to obtain

$$
\begin{aligned}
\int_{\Gamma} & \left(\left\langle D^{\prime}\right\rangle^{0.5}\left[\partial^{\beta}(p+\mu \dot{p})\right]\right)\left(\left\langle D^{\prime}\right\rangle^{0.5} \partial_{x_{i}} \partial^{\alpha} f\right) \mathrm{d} x^{\prime} \\
= & \sigma \int_{\Gamma}\left(\left\langle D^{\prime}\right\rangle^{0.5} \partial^{\alpha}\left(\frac{\nabla^{\prime} f}{|n|}\right)\right) \cdot\left(\left\langle D^{\prime}\right\rangle^{0.5} \partial^{\alpha} \nabla^{\prime} f\right) \mathrm{d} x^{\prime} \\
& +\mu \int_{\Gamma}\left(\left\langle D^{\prime}\right\rangle^{0.5} \partial^{\alpha} \nabla^{\prime} \partial_{t} f\right)\left(\left\langle D^{\prime}\right\rangle^{0.5} \partial^{\alpha} \nabla^{\prime} f\right) \mathrm{d} x^{\prime} \\
& +\mu \int_{\Gamma}\left(\left\langle D^{\prime}\right\rangle^{0.5} \partial^{\alpha} \partial_{t} f\right)\left(\left\langle D^{\prime}\right\rangle^{0.5} \partial^{\alpha} f\right) \mathrm{d} x^{\prime}+\int_{\Gamma}\left(\left\langle D^{\prime}\right\rangle^{0.5} R_{\Gamma}^{2}(f)\right)^{2} \mathrm{~d} x^{\prime}
\end{aligned}
$$

where the remainder $R_{\Gamma}^{2}(f)$ is defined in Definition 31. We now use Lemma 6 to differentiate $\frac{\nabla^{\prime} f}{|n|}$, together with the fractional product rule given in Lemma 31 from the appendix, and following the notation of this lemma, we denote the remainder term by $R\left(G\left(\nabla^{\prime} f\right), \partial^{\alpha} \nabla^{\prime} f\right)$, where $G$ is a smooth function. We obtain

$$
\begin{aligned}
& \int_{\Gamma}\left(\left\langle D^{\prime}\right\rangle^{0.5}\left[\partial^{\beta}(p+\mu \dot{p})\right]\right)\left(\left\langle D^{\prime}\right\rangle^{0.5} \partial^{\alpha} \partial_{x_{i}} f\right) \mathrm{d} x^{\prime} \\
&=\sigma \int_{\Gamma} \frac{1}{|n|}\left|\left\langle D^{\prime}\right\rangle^{0.5} \partial^{\alpha} \nabla^{\prime} f\right|^{2}-\frac{1}{|n|^{3}}\left|\nabla^{\prime} f \cdot\left\langle D^{\prime}\right\rangle^{0.5} \partial^{\alpha} \nabla^{\prime} f\right|^{2} \mathrm{~d} x^{\prime} \\
& \quad+\sigma \int_{\Gamma}\left\langle D^{\prime}\right\rangle^{0.5} R_{\Gamma}^{2}(f) \cdot\left\langle D^{\prime}\right\rangle^{0.5} \partial^{\alpha} \nabla^{\prime} f \mathrm{~d} x^{\prime} \\
& \quad+\sigma \int_{\Gamma} R\left(G\left(\nabla^{\prime} f\right), \partial^{\alpha} \nabla^{\prime} f\right) \cdot\left\langle D^{\prime}\right\rangle^{0.5} \partial^{\alpha} \nabla^{\prime} f \mathrm{~d} x^{\prime} \\
& \quad+\mu \frac{1}{2} \frac{\mathrm{d}}{\mathrm{d} t} \int_{\Gamma}\left|\left\langle D^{\prime}\right\rangle^{0.5} \partial^{\alpha} \nabla^{\prime} f\right|^{2} \mathrm{~d} x^{\prime} \\
& \quad+\int_{\Gamma}\left(\left\langle D^{\prime}\right\rangle^{0.5} \partial_{t} R_{\Gamma}^{2}(f)\right)\left\langle D^{\prime}\right\rangle^{0.5} R_{\Gamma}^{2}(f) \mathrm{d} x^{\prime}+\int_{\Gamma}\left(\left\langle D^{\prime}\right\rangle^{0.5} R_{\Gamma}^{2}(f)\right)^{2} \mathrm{~d} x^{\prime} \\
& \geqq \sigma \int_{\Gamma} \frac{1}{|n|^{3}}\left|\left\langle D^{\prime}\right\rangle^{0.5} \partial^{\alpha} \nabla^{\prime} f\right|^{2} \mathrm{~d} x^{\prime}-\frac{1}{\delta}\left\|R\left(G\left(\nabla^{\prime} f\right), \partial^{\alpha} \nabla^{\prime} f\right)\right\|_{L^{2}(\Gamma)}^{2} \\
&-\delta \int_{\Gamma}\left|\left\langle D^{\prime}\right\rangle^{0.5} \partial^{\alpha} \nabla^{\prime} f\right|^{2} \mathrm{~d} x^{\prime}+\mu \frac{1}{2} \frac{\mathrm{d}}{\mathrm{d} t} \int_{\Gamma}\left|\left\langle D^{\prime}\right\rangle^{0.5} \partial^{\alpha} \nabla^{\prime} f\right|^{2} \mathrm{~d} x^{\prime} \\
&-\left\|R_{\Gamma}^{2}(f)\right\|_{H^{1}(\Gamma)}^{2}-\left\|\partial_{t} R_{\Gamma}^{2}(f)\right\|_{L^{2}(\Gamma)}^{2}-C\left\|\partial^{\alpha} \nabla^{\prime} f\right\|_{L^{2}(\Gamma)}^{2},
\end{aligned}
$$

where we have used Lemma 7. We have also used Cauchy's inequality in the last step, and we are free to choose $0<\delta \leqq 1$. Note that $\partial R_{\Gamma}^{2}(f)$ is a sum of terms of the form $G\left(\nabla^{\prime} f\right)\left(\partial \partial^{\gamma}\left(\nabla^{\prime} f, g_{2}\right)+\partial \nabla^{\prime} f R^{2}(f)\right)$ where $|\gamma| \leqq 2$ and $G$ is a smooth function. We recall that we have already estimated $\partial \partial^{\gamma} \nabla^{\prime} f$ in (88). Hence, using 
Cauchy's inequality, the remainder estimate (123), and the estimate of the remainder from Lemma 31, we obtain

$$
\begin{aligned}
& \sigma \int_{\Gamma} \frac{1}{|n|^{3}}\left|\left\langle D^{\prime}\right\rangle^{0.5} \partial^{\alpha} \nabla^{\prime} f\right|^{2} \mathrm{~d} x^{\prime}+\frac{1}{2} \mu \frac{\mathrm{d}}{\mathrm{d} t} \int_{\Gamma}\left|\left\langle D^{\prime}\right\rangle^{0.5} \partial^{\alpha} \nabla^{\prime} f\right|^{2} \mathrm{~d} x^{\prime} \\
& \leqq \\
& \leq \frac{C}{\delta}\left\|\left\langle D^{\prime}\right\rangle^{0.5}\left[\partial^{\beta}(p+\mu \dot{p})\right]\right\|_{L^{2}(\Gamma)}^{2}+\delta\left\|\left\langle D^{\prime}\right\rangle^{0.5} \partial^{\alpha} \nabla^{\prime} f\right\|_{L^{2}(\Gamma)}^{2} \\
& \quad+\frac{1}{\delta}\left(M^{0}+t K(t)\right)\left(\frac{1}{\varepsilon^{\frac{3}{2}}}+\varepsilon^{\frac{1}{2}} E_{\mu}(t)\right) .
\end{aligned}
$$

We choose $\delta=\frac{\sigma}{2\|n\|_{L^{\infty}(\Gamma)}^{3}}$ and rearrange and use the Sobolev trace theorem to obtain

$$
\begin{aligned}
& \sigma \int_{\Gamma} \frac{1}{|n|^{3}}\left|\left\langle D^{\prime}\right\rangle^{0.5} \partial^{\alpha} \nabla^{\prime} f\right|^{2} \mathrm{~d} x^{\prime}+\mu \frac{\mathrm{d}}{\mathrm{d} t} \int_{\Gamma}\left|\left\langle D^{\prime}\right\rangle^{0.5} \partial^{\alpha} \nabla^{\prime} f\right|^{2} \mathrm{~d} x^{\prime} \\
& \quad \leqq C \sum_{ \pm} \frac{\|n\|_{L^{\infty}(\Gamma)}^{3}}{\sigma}\left\|\partial^{\beta}(p+\mu \dot{p})\right\|_{H^{1}\left(\Omega_{ \pm}\right)}^{2}+\left(M^{0}+t K(t)\right)\left(\frac{1}{\varepsilon^{\frac{3}{2}}}+\varepsilon^{\frac{1}{2}} E_{\mu}(t)\right) \\
& \quad \leqq\left(M^{0}+t K(t)\right)\left(\frac{1}{\varepsilon^{\frac{3}{2}}}+\varepsilon^{\frac{1}{2}} E_{\mu}(t)\right)
\end{aligned}
$$

where we have used the lower order estimate (64) and the existing energy estimates (83), (101) and (102). Now we apply the Lemma 8 to obtain

$$
\int_{\Gamma}\left|\left\langle D^{\prime}\right\rangle^{0.5} \partial^{\alpha} \nabla^{\prime} f\right|^{2} \mathrm{~d} x^{\prime} \leqq\left(M^{0}+t K(t)\right)\left(\frac{1}{\varepsilon^{\frac{3}{2}}}+\varepsilon^{\frac{1}{2}} E_{\mu}(t)\right) .
$$

This completes the proof.

\subsection{Estimate of the Additional Terms in the Energy Involving $\mu$}

The following estimates are in fact not essential for the closure of the energy estimate (with an energy involving fewer $\mu$-dependent terms, although we have included the extra terms in the energy for convenience later on), so we will skip most of the details.

By applying $\partial^{\alpha}$ where $|\alpha|=3$ and $\alpha_{0} \leqq 2$ to the curl equation (54) and multiplying by $\mu^{2} \partial^{\alpha}\left(\nabla^{\psi} \times u\right)$ then integrating in space and time and using existing estimates where necessary, we obtain the estimate

$$
\mu^{2}\left\|\nabla^{\psi} \times \partial^{\alpha} u\right\|^{2} \leqq\left(M^{0}+t K(t)\right)\left(\frac{1}{\varepsilon^{\frac{3}{2}}}+\varepsilon^{\frac{1}{2}} E_{\mu}(t)\right)
$$

for $|\alpha|=2$. Note that we need to use the fact that we have mollified the coefficient $\rho$ to give $\rho_{\mu}$, and that $\left\|\nabla \rho_{\mu}\right\|_{L^{2}\left(\Omega_{ \pm}\right)} \leqq C \frac{1}{\mu}\|\rho\|_{L^{2}\left(\Omega_{ \pm}\right)}$.

Applying $\partial^{\alpha}$ where $|\alpha|=2$ to the curl equation (54), we obtain

$$
\rho_{\mu} \nabla^{\psi} \times \partial_{t} \partial^{\alpha} u=-\rho_{\mu}\left(u^{\psi} \cdot \nabla\right) \nabla^{\psi} \times \partial^{\alpha} u+R^{3}(U, \psi) .
$$


Multiplying by $\mu$, squaring, integrating in time and using the remainder estimate (118), we obtain

$$
\mu^{2} \int_{0}^{t}\left\|\nabla^{\psi} \times \partial_{t} \partial^{\alpha} u\right\|^{2} \mathrm{~d} \tau \leqq t K(t)
$$

for $|\alpha|=2$.

Now we obtain an elliptic-type estimate for $u \cdot n$ similar to the elliptic estimate we obtained for $f$. First we prove a very simple lemma which we will reuse later.

Lemma 11. Let $z \in H^{1}(\Gamma)$ and $h \in H^{s}(\Gamma)$ for $s \in \mathbb{R}_{\geq_{0}}$ with

$$
\left(1-\Delta^{\prime}\right) z=h
$$

on $\Gamma$ in the sense of distributions. Then $z \in H^{s+2}(\Gamma)$ with

$$
\|z\|_{H^{s+2}(\Gamma)} \leqq C\|h\|_{H^{s}(\Gamma)} \text {. }
$$

Proof. Taking Fourier transforms with respect to $x^{\prime}$, we have

$$
\left\langle\xi^{\prime}\right\rangle^{2} \hat{z}=\hat{h} .
$$

Multiplying by $\left\langle\xi^{\prime}\right\rangle^{S}$, then squaring, we obtain

$$
\left|\left\langle\xi^{\prime}\right\rangle^{s+2} \hat{z}\right|^{2}=\left|\left\langle\xi^{\prime}\right\rangle^{s} \hat{h}\right|^{2}
$$

Integrating over $\mathbb{R}^{2}$ and using the definition of the Sobolev norm in terms of Fourier transforms, we obtain $z \in H^{s+2}(\Gamma)$ with

$$
\|z\|_{H^{s+2}(\Gamma)}^{2}=\|h\|_{H^{s}(\Gamma)}^{2}
$$

as required.

Now we let $0 \leqq j \leqq 2$ and apply $\partial_{t}^{j}$ to the pressure interface condition (43). We obtain

$$
\mu\left(1-\Delta^{\prime}\right) \partial_{t}^{j}(u \cdot n)=\partial_{t}^{j}\left(-[p]-\sigma \nabla^{\prime} \cdot \hat{n}+g_{2}-\mu[\dot{p}]\right) .
$$

We apply the above lemma with

$$
\begin{aligned}
& z=\mu \partial_{t}^{j}(u \cdot n) \\
& h=\partial_{t}^{j}\left(-[p]-\sigma \nabla^{\prime} \cdot \hat{n}+g_{2}-\mu[\dot{p}]\right)
\end{aligned}
$$

and the Sobolev trace estimate to conclude that

$$
\mu^{2}\left\|\partial_{t}^{j}(u \cdot n)\right\|_{H^{4.5-j}(\Gamma)}^{2} \leqq\left(M^{0}+t K(t)\right)\left(\frac{1}{\varepsilon^{\frac{3}{2}}}+\varepsilon^{\frac{1}{2}} E_{\mu}(t)\right),
$$

where we have used the chain and product rules, the lower order estimates (62), (64), (65) and the existing estimates (83), (88), (92), (101), (100), (89) and (103). 
Let $1 \leqq|\alpha| \leqq 3$ with $\alpha_{3}=0$. We apply the Hodge decomposition estimate given in Proposition 36 along with the lower order estimate (64) to obtain

$$
\begin{aligned}
\left\|\partial^{\alpha} u\right\|_{H^{1}\left(\Omega_{ \pm}\right)}^{2} \leqq & \left(M^{0}+t K(t)\right)\left(\left\|\partial^{\alpha} u\right\|_{L^{2}\left(\Omega_{ \pm}\right)}^{2}+\left\|\nabla^{\psi} \cdot \partial^{\alpha} u\right\|_{L^{2}\left(\Omega_{ \pm}\right)}^{2}\right. \\
& \left.+\left\|\nabla^{\psi} \times \partial^{\alpha} u\right\|_{L^{2}\left(\Omega_{ \pm}\right)}^{2}+\left\|\partial^{\alpha} u \cdot n\right\|_{H^{0.5}(\Gamma)}^{2}\right) \\
\leqq & \left(M^{0}+t K(t)\right)\left(\left\|\partial^{\alpha} u\right\|_{L^{2}\left(\Omega_{ \pm}\right)}^{2}+\left\|\nabla^{\psi} \cdot \partial^{\alpha} u\right\|_{L^{2}\left(\Omega_{ \pm}\right)}^{2}\right. \\
& +\left\|\nabla^{\psi} \times \partial^{\alpha} u\right\|_{L^{2}\left(\Omega_{ \pm}\right)}^{2} \\
& +\left\|\partial^{\alpha}(u \cdot n)\right\|_{H^{0.5}(\Gamma)}^{2}+\left\|u \cdot \partial^{\alpha} n\right\|_{H^{0.5}(\Gamma)}^{2} \\
& \left.+\sum_{\beta+\gamma=\alpha, \beta \neq \alpha, \gamma \neq \alpha}\left\|\partial^{\beta} u \cdot \partial^{\gamma} n\right\|_{H^{0.5}(\Gamma)}^{2}\right) .
\end{aligned}
$$

Now in the case $\alpha_{0} \leqq 2$, we multiply by $\mu^{2}$ apply the existing estimates (103), (105), (83), (92), (100), (104) and the Sobolev embedding theorem to obtain

$$
\mu^{2}\left\|\partial^{\alpha} u\right\|_{H^{1}\left(\Omega_{ \pm}\right)}^{2} \leqq\left(M^{0}+t K(t)\right)\left(\frac{1}{\varepsilon^{\frac{3}{2}}}+\varepsilon^{\frac{1}{2}} E_{\mu}(t)\right) .
$$

In the case $\alpha_{0}=3$, we multiply by $\mu^{2}$, integrate in time, apply the existing estimates (106), (83), (92), (100), (104) and the Sobolev embedding theorem, and rewrite the term $\left\|u \cdot \partial^{\alpha} n\right\|_{H^{0.5}(\Gamma)}^{2}$ as $\left\|u \cdot \partial_{t}^{2} \nabla^{\prime}(u \cdot n)\right\|_{H^{0.5}(\Gamma)}^{2}$ to obtain

$$
\mu^{2} \int_{0}^{t}\left\|\partial^{\alpha} u\right\|_{H^{1}\left(\Omega_{ \pm}\right)}^{2} \mathrm{~d} \tau \leqq\left(M^{0}+t K(t)\right)\left(\frac{1}{\varepsilon^{\frac{3}{2}}}+\varepsilon^{\frac{1}{2}} E_{\mu}(t)\right)
$$

Now it remains to estimate $\left\|\partial^{\alpha} u\right\|_{H^{1}\left(\Omega_{ \pm}\right)}$for $0 \leqq|\alpha| \leqq 3$ where $\alpha_{3} \geqq 1$, but we may apply Lemma 9 to obtain, for $|\alpha|=3$ and $\alpha_{0} \leqq 2$ :

$$
\partial_{x_{3}} \partial^{\alpha} u_{i}=G_{i}(\nabla \psi) \cdot\left(\partial_{x_{1}} \partial^{\alpha} u, \partial_{x_{2}} \partial^{\alpha} u, \nabla^{\psi} \cdot \partial^{\alpha} u, \nabla^{\psi} \times \partial^{\alpha} u\right),
$$

where $G_{i}(\nabla \psi)$ is a smooth vector-valued function for each $i=1,2,3$. We claim inductively on $\alpha_{3}$ for $0 \leqq \alpha_{3} \leqq 3$ that

$$
\mu^{2}\left\|\partial_{x_{3}} \partial^{\alpha} u_{i}\right\|_{L^{2}\left(\Omega_{ \pm}\right)}^{2} \leqq\left(M^{0}+t K(t)\right)\left(\frac{1}{\varepsilon^{\frac{3}{2}}}+\varepsilon^{\frac{1}{2}} E_{\mu}(t)\right) .
$$

Indeed, we have already proved the case $\alpha_{3}=0$. So we assume it is true for $\alpha_{3} \leqq k$ where $0 \leqq k \leqq 2$ and try to prove it is true for $\alpha_{3}=k+1$. Using the above formula we have

$$
\begin{aligned}
& \mu^{2}\left\|\partial_{x_{3}} \partial^{\alpha} u_{i}\right\|_{L^{2}\left(\Omega_{ \pm}\right)}^{2} \\
& \quad \leqq \mu^{2}\left(M^{0}+t K(t)\right)\left(\left\|\nabla^{\prime} \partial^{\alpha} u\right\|_{L^{2}\left(\Omega_{ \pm}\right)}^{2}+\left\|\nabla^{\psi} \cdot \partial^{\alpha} u\right\|_{L^{2}\left(\Omega_{ \pm}\right)}^{2}+\left\|\nabla^{\psi} \times \partial^{\alpha} u\right\|_{L^{2}\left(\Omega_{ \pm}\right)}^{2}\right) \\
& \quad \leqq\left(M^{0}+t K(t)\right)\left(\frac{1}{\varepsilon^{\frac{3}{2}}}+\varepsilon^{\frac{1}{2}} E_{\mu}(t)\right),
\end{aligned}
$$

where we have used the induction hypothesis for the first term on the right, the estimate (103) for the second term on the right and the estimate (105) for the third 
term on the right. This completes the proof of the induction step. Hence we have finally proved that

$$
\mu^{2}\left\|\partial^{\alpha} u\right\|_{H^{1}\left(\Omega_{ \pm}\right)}^{2} \leqq\left(M^{0}+t K(t)\right)\left(\frac{1}{\varepsilon^{\frac{3}{2}}}+\varepsilon^{\frac{1}{2}} E_{\mu}(t)\right)
$$

for $1 \leqq|\alpha| \leqq 3$ with $\alpha_{0} \leqq 2$.

\subsection{Conclusion of the Energy Estimate}

Combining the estimates (62), (64), (65), (81), (83), (92), (88), (89), (100), (101), (104), (109), (108) and (107) we obtain

$$
E_{\mu}(t) \leqq\left(M^{0}+t K(t)\right)\left(\frac{1}{\varepsilon^{\frac{3}{2}}}+\varepsilon^{\frac{1}{2}} E_{\mu}(t)\right) .
$$

Now for each $t$ we may choose $\varepsilon^{\frac{1}{2}}=\frac{1}{2\left(M^{0}+t K(t)\right)}$ and rearrange to obtain

$$
E_{\mu}(t) \leqq M^{0}+t K(t),
$$

where we have redefined $M^{0}$ and $K(t)$. From this, the result of Proposition 7 follows from a well-known argument which proceeds by setting

$$
\begin{aligned}
T^{0}=\sup \left\{t \in\left(0, T^{\prime}\right): E_{\mu}(t) \leqq 2 M^{0},\right. & \inf _{\tau \in(0, t)} \inf _{\Omega_{ \pm}} \rho \geqq \frac{2 \delta^{0}}{3}, \\
& \left.\inf _{\tau \in(0, t)} \inf _{\Omega_{ \pm}} \rho_{\mu} \geqq \frac{2 \delta_{\mu}^{0}}{3}, \inf _{\tau \in(0, t)} \inf _{\mathbb{R}^{3}} J^{\psi} \geqq \frac{2 \kappa^{0}}{3}\right\},
\end{aligned}
$$

and using continuity together with the inequalities (59)-(61) and (110) evaluated at $t=T^{0}$ to obtain a lower bound of the desired form for $T^{0}$.

\subsection{Remainder Estimates}

One of the key observations in deriving high-order energy estimates is that once we have differentiated the equations enough times, the lower order terms may all be estimated in terms of the energy by the Sobolev embedding theorem, and hence we may try and obtain energy estimates in the same way as for constant-coefficient linear equations. To make the analysis as clear as possible, in this section we define the types of generic remainder term which are generated and prove estimates for them, so that we can concentrate on dealing with the important higher-order terms in the main body of the proof of the energy estimate. We have only sketched the proofs in this section as they are generally straightforward but technical.

Remark 4. We note that $U_{+}$can be extended to $(0, T) \times \Omega_{-}$in such a way that the Sobolev norm of the extended function is controlled by a constant times the Sobolev norm of the original function, and similarly $U_{-}$can be extended to $(0, T) \times \Omega_{+}$. Hence in the following section on remainders, we will write $U$ when we could be referring to either $U_{+}$or $U_{-}$, or their extensions to $(0, T) \times \Omega_{-}$or $(0, T) \times \Omega_{+}$. We can do this because the estimates only depend on a control of their Sobolev norms, and our energy functional is symmetric with respect to \pm terms. 
We start with an estimate of $\dot{p}$ in terms of the energy.

Lemma 12. Let $0 \leqq|\beta| \leqq 2$ and $0 \leqq|\alpha| \leqq 3$. We have

$$
\begin{aligned}
\mu^{2}\left\|\partial^{\beta} \dot{p}\right\|_{H^{1}\left(\Omega_{ \pm}\right)}^{2} & \leqq\left(M^{0}+t K(t)\right) E_{\mu}(t) \\
\mu^{2} \int_{0}^{t}\left\|\partial^{\alpha} \dot{p}\right\|_{L^{2}\left(\Omega_{ \pm}\right)}^{2} \mathrm{~d} \tau & \leqq\left(M^{0}+t K(t)\right) E_{\mu}(t) .
\end{aligned}
$$

Proof. We use the fact that

$$
\mu \dot{p}=-\mu \rho c^{2} \nabla^{\psi} \cdot u
$$

together with the chain rule, the Sobolev embedding theorem and the Sobolev product lemma, Lemma 30.

Now we define the remainder terms.

Definition 27. Let $0 \leqq k \leqq 1$. We will write $R_{\infty}^{k}(U, \psi)$ for a generic remainder term of the form

$$
R_{\infty}^{k}(U, \psi)=G\left(\left\{\partial^{\beta}\left(U, \rho_{\mu}, \psi, \partial \psi, g_{1}\right):|\beta| \leqq k\right\}\right),
$$

where $G$ is a smooth function of its arguments in the region $\rho>0, \rho_{\mu}>0, J^{\psi}>0$. Similarly, for $0 \leqq k \leqq 1$, we will write $R_{\infty, \Gamma}^{k}(U, f)$ for a generic remainder term of the form

$$
R_{\infty, \Gamma}^{k}(U, \psi)=G\left(\left\{\partial^{\beta}\left(\left.U\right|_{\Gamma}, f, \partial^{\prime} f\right):|\beta| \leqq k\right\}\right),
$$

where $G$ is a smooth function of its arguments. We will also write $R_{\infty, \Gamma}(f)$ for a generic remainder term of the form

$$
R_{\infty, \Gamma}(f)=G\left(\nabla^{\prime} f, g_{2}\right)
$$

where $G$ is a smooth function of its arguments.

Definition 28. Let $0 \leqq k \leqq 3$. We will write $R^{k}(U, \psi)$ for a generic remainder term which consists of sums of terms of the form

$$
G\left(\left\{\partial_{x_{3}}^{j} \psi: 0 \leqq j \leqq k+1\right\}\right) R_{\infty}^{0}(U, \psi) \prod_{i=1}^{n} \partial^{\alpha^{i}}\left(U, \rho_{\mu}, \nabla^{\prime} \psi, \partial_{t} \psi, g_{1}\right)_{e(i)}
$$

(which is not allowed in the case $k=0$ ) and

$$
G\left(\left\{\partial_{x_{3}}^{j} \psi: 0 \leqq j \leqq k+1\right\}\right) R_{\infty}^{0}(U, \psi) \cdot \partial^{\alpha} g_{1} .
$$

Here, $n$ is an integer with $1 \leqq n \leqq k+1, \alpha^{i}$ are multi-indices with $0 \leqq\left|\alpha^{i}\right| \leqq$ $k$ and $1 \leqq \sum_{i=1}^{n}\left|\alpha^{i}\right| \leqq k+1,\left(U, \rho_{\mu}, \nabla^{\prime} \psi, \partial_{t} \psi, g_{1}\right)_{e(i)}$ denotes an entry of $\left(U, \rho_{\mu}, \nabla^{\prime} \psi, \partial_{t} \psi, g_{1}\right)$ depending on $i, R_{\infty}^{0}(U, \psi)$ is defined in Definition 27, $G$ is a smooth function of its arguments, and $|\alpha| \leqq k$. Finally, we impose the important constraint that if we have $\left(U, \rho_{\mu}, \nabla^{\prime} \psi, \partial_{t} \psi, g_{1}\right)_{e(i)}=\partial_{t} \psi$ then $\left|\alpha^{i}\right| \leqq k-1$ or $\partial^{\alpha^{i}}$ contains at least one space derivative (so it is not all time derivatives). 
Definition 29. Let $0 \leqq k \leqq 3$. We will write $R^{k}(U, \psi, \dot{p})$ for a generic remainder term which consists of sums of terms of the form

$$
R^{k}(U, \psi)
$$

or

$$
G\left(\left\{\partial_{x_{3}}^{j} \psi: 0 \leqq j \leqq k+1\right\}\right) R_{\infty}^{0}(U, \psi) \cdot\left(\prod_{i=1}^{n} \partial^{\alpha^{i}}\left(U, \rho_{\mu}, \nabla^{\prime} \psi, g\right)_{e(i)}\right) \partial^{\beta} \dot{p} .
$$

Here, $n$ is an integer with $0 \leqq n \leqq k+1, \alpha^{i}$ are multi-indices with $0 \leqq\left|\alpha^{i}\right| \leqq k, \beta$ is a multi-index with $0 \leqq|\beta| \leqq k$ and $0 \leqq \sum_{i=1}^{n}\left|\alpha^{i}\right|+|\beta| \leqq k,\left(U, \rho_{\mu}, \nabla^{\prime} \psi, g\right)_{e(i)}$ denotes an entry of $\left(U, \rho_{\mu}, \nabla^{\prime} \psi, g\right)$ depending on $i, R_{\infty}^{0}(U, \psi)$ is defined in Definition 27, and $G$ is a smooth function of its arguments.

Definition 30. Let $1 \leqq k \leqq 2$. We will write $R_{\Gamma}^{k}(U, f)$ for a generic remainder term which consists of sums of terms of the form

$$
R_{\infty, \Gamma}^{0}(U, f) \prod_{i=1}^{n} \partial^{\alpha^{i}}\left(\left.U\right|_{\Gamma}, \partial^{\prime} f\right)_{e(i)}
$$

Here, $n$ is an integer with $1 \leqq n \leqq k+1, \alpha^{i}$ are multi-indices with $\alpha_{3}^{i}=0$, $1 \leqq\left|\alpha^{i}\right| \leqq k$ and $\sum_{i=1}^{n}\left|\alpha^{i}\right| \leqq k+1,\left(\left.U\right|_{\Gamma}, \partial^{\prime} f\right)_{e(i)}$ denotes an entry of $\left(\left.U\right|_{\Gamma}, \partial^{\prime} f\right)$ depending on $i$, and $R_{\infty, \Gamma}^{0}(U, f)$ is defined in Definition 27.

We may sometimes write $R_{\Gamma}^{0}(U, f)$, which is defined to be zero.

Definition 31. Let $1 \leqq k \leqq 3$. We will write $R_{\Gamma}^{k}(f)$ for a generic remainder term which consists of sums of terms of the form

$$
R_{\infty, \Gamma}(f) \prod_{i=1}^{n} \partial^{\alpha^{i}}\left(\nabla^{\prime} f, g_{2}\right)_{e(i)}
$$

Here, $n$ is an integer with $1 \leqq n \leqq k+1, \alpha^{i}$ are multi-indices with $\alpha_{3}^{i}=0$, $1 \leqq\left|\alpha^{i}\right| \leqq k$ and $\sum_{i=1}^{n}\left|\alpha^{i}\right| \leqq k+1,\left(\nabla^{\prime} f, g_{2}\right)_{e(i)}$ denotes an entry of $\left(\nabla^{\prime} f, g_{2}\right)$ depending on $i$, and $R_{\infty, \Gamma}(f)$ is defined in Definition 27.

We may sometimes write $R_{\Gamma}^{0}(f)$, which is defined to be zero.

Now we provide estimates for the remainder terms.

Lemma 13. For $t \in\left(0, T^{\prime}\right)$, the remainder terms $R_{\infty}^{k}(U, \psi), R_{\infty, \Gamma}^{k}(U, f)$ and $R_{\infty, \Gamma}(f)$ satisfy

$$
\begin{aligned}
\left\|R_{\infty}^{k}(U, \psi)\right\|_{L^{\infty}\left(\Omega_{ \pm}\right)}^{2} & \leqq K(t) \text { for } 0 \leqq k \leqq 1 \\
\left\|R_{\infty, \Gamma}^{k}(U, f)\right\|_{L^{\infty}(\Gamma)}^{2} & \leqq K(t) \text { for } 0 \leqq k \leqq 1 \\
\left\|R_{\infty, \Gamma}(f)\right\|_{L^{\infty}(\Gamma)}^{2} & \leqq K(t) \\
\left\|R_{\infty}^{k}(U, \psi)\right\|_{L^{\infty}\left(\Omega_{ \pm}\right)}^{2} & \leqq M^{0}+t K(t) \text { for } k=0 \\
\left\|R_{\infty, \Gamma}^{k}(U, f)\right\|_{L^{\infty}(\Gamma)}^{2} & \leqq M^{0}+t K(t) \text { for } k=0 .
\end{aligned}
$$


Proof. We simply use the Sobolev embedding theorem, plus uniform continuity for the second estimate, and the fundamental theorem of calculus for the last two estimates.

Lemma 14. Given $t \in\left(0, T^{\prime}\right)$, the remainder terms $R^{k}(U, \psi), R^{k}(U, \psi, \dot{p})$, $R_{\Gamma}^{k}(U, f)$ and $R_{\Gamma}^{k}(f)$ satisfy the following estimates.

$$
\begin{aligned}
\left\|R^{k}(U, \psi)\right\|_{L^{2}\left(\Omega_{ \pm}\right)}^{2} & \leqq K(t) \text { for } 0 \leqq k \leqq 3 \\
\left\|R_{\Gamma}^{k}(U, f)\right\|_{L^{2}(\Gamma)}^{2} & \leqq K(t) \text { for } 0 \leqq k \leqq 2 \\
\left\|R_{\Gamma}^{k}(f)\right\|_{L^{2}(\Gamma)}^{2} & \leqq K(t) \text { for } 0 \leqq k \leqq 3 \\
\left\|R^{k}(U, \psi)\right\|_{L^{2}\left(\Omega_{ \pm}\right)}^{2} & \leqq M^{0}+t K(t) \text { for } 0 \leqq k \leqq 2 \\
\left\|R_{\Gamma}^{k}(U, f)\right\|_{L^{2}(\Gamma)}^{2} & \leqq M^{0}+t K(t) \text { for } 0 \leqq k \leqq 1 \\
\left\|R_{\Gamma}^{k}(f)\right\|_{L^{2}(\Gamma)}^{2} & \leqq M^{0}+t K(t) \text { for } 0 \leqq k \leqq 2 .
\end{aligned}
$$

Also, for $0 \leqq k \leqq 3$ and $1 \leqq|\alpha| \leqq 3$, we have

$$
\mu \int_{0}^{t} \int_{\Omega_{ \pm}}\left|R^{k}(U, \psi, \dot{p}) \cdot \partial^{\alpha} U\right| \mathrm{d} x \mathrm{~d} \tau \leqq \frac{1}{\varepsilon} t K(t)+\varepsilon\left(M^{0}+t K(t)\right) E_{\mu}(t)
$$

where we are free to choose $\varepsilon$ in the range $0<\varepsilon \leqq 1$.

Finally, let $0 \leqq k \leqq 2$, and let $\alpha$ be a multi-index with $\alpha_{3} \leqq|\alpha|-1$ and $1 \leqq|\alpha| \leqq 3$. Thus we have either $\partial^{\alpha}=\partial_{x_{j}} \partial^{\beta}$ for some $1 \leqq j \leqq 2$ and $|\beta|=|\alpha|-1$, or $\partial^{\alpha}=\partial_{t} \partial^{\beta}$ for some $|\beta|=|\alpha|-1$. Then

$$
\left|\int_{0}^{t} \int_{\Gamma} R_{\Gamma}^{k}(U, f) \partial^{\alpha}(U, \mu \dot{p}) \mathrm{d} x^{\prime} \mathrm{d} \tau\right| \leqq\left(M^{0}+t K(t)\right)\left(\frac{1}{\varepsilon}+\varepsilon E_{\mu}(t)\right),
$$

where we are free to choose $\varepsilon$ in the range $0<\varepsilon \leqq 1$.

Proof. The estimates (121)-(123) follow from (118)-(120) using the fundamental theorem of calculus.

To prove (118), we use (113), plus the Sobolev product lemma, Lemma 30, applied to the product which occurs in the remainder, $\prod_{i=1}^{n} \partial^{\alpha^{i}}\left(U, \rho_{\mu}, \nabla^{\prime} \psi, \partial_{t} \psi\right.$, $\left.g_{1}\right)_{e(i)}$. We do this by setting $m_{i}=3-\left|\alpha^{i}\right|$ and $m=2>\frac{3}{2}=\frac{d}{p}$ and checking $\sum_{i=1}^{n} m_{i}=3 n-\sum_{i=1}^{n}\left|\alpha^{i}\right| \geqq(n-1) m$. We conclude by using the definition of the energy.

The proof of (124) is similar, except we obtain

$$
\left\|R^{k}(U, \psi, \dot{p})\right\|_{L^{2}\left(\Omega_{ \pm}\right)} \leqq K(t) \sum_{ \pm} \sum_{j=0}^{3}\left\|\partial_{t}^{j} \dot{p}\right\|_{H^{3-j}\left(\Omega_{ \pm}\right)}
$$

Applying Cauchy's inequality we obtain the desired estimate, where we have used the estimates (111)-(112) for $\dot{p}$. 
To prove (119), we note that since $\partial_{t} f=u \cdot n=u_{3}-u^{\prime} \cdot \nabla^{\prime} f$ by (42), we can in fact write $R_{\Gamma}^{k}(U, f)$ as a sum of terms of the form

$$
R_{\infty, \Gamma}^{0}(U, f) \prod_{i=1}^{n} \partial^{\alpha^{i}}\left(\left.U\right|_{\Gamma}, \nabla^{\prime} f\right)_{e(i)} .
$$

We note that in fact this has the form

$$
\left.R^{k}(U, \psi)\right|_{\Gamma}
$$

where $R^{k}(U, \psi)$ is defined in Definition 28. Hence, using the Sobolev trace lemma,

$$
\left\|R_{\Gamma}^{k}(U, f)\right\|_{L^{2}(\Gamma)}^{2} \leqq\left\|R^{k}(U, f)\right\|_{H^{1}\left(\Omega_{ \pm}\right)}^{2} \leqq\left\|R^{k+1}(U, f)\right\|_{L^{2}\left(\Omega_{ \pm}\right)}^{2} \leqq K(t),
$$

where we have used (118).

To prove (125), we convert the above to an integral on the left hand side to one over the interior. We have

$$
\begin{aligned}
& \left|\int_{0}^{t} \int_{\Gamma} R_{\Gamma}^{k}(U, f) \partial^{\alpha}(U, \mu \dot{p}) \mathrm{d} x^{\prime} \mathrm{d} \tau\right| \\
& \quad \leqq \sum_{ \pm}\left|\int_{0}^{t} \int_{\Omega_{ \pm}} \partial_{x_{3}}\left(R^{k}(U, \psi) \partial^{\alpha}(U, \mu \dot{p})\right) \mathrm{d} x \mathrm{~d} \tau\right| \\
& \quad \leqq \sum_{ \pm}\left|\int_{0}^{t} \int_{\Omega_{ \pm}} R^{k+1}(U, \psi) \partial^{\alpha}(U, \mu \dot{p})+R^{k}(U, \psi) \partial^{\alpha} \partial_{x_{3}}(U, \mu \dot{p}) \mathrm{d} x \mathrm{~d} \tau\right| \\
& \quad \leqq \sum_{ \pm}\left|\int_{0}^{t} \int_{\Omega_{ \pm}} R^{k}(U, \psi) \partial^{\alpha} \partial_{x_{3}}(U, \mu \dot{p}) \mathrm{d} x \mathrm{~d} \tau\right|+\frac{1}{\varepsilon} \int_{0}^{t}\left\|R^{k+1}(U, \psi)\right\|_{L^{2}\left(\Omega_{ \pm}\right)}^{2} \mathrm{~d} \tau \\
& \quad+\varepsilon \sum_{ \pm} \mu^{2} \int_{0}^{t}\left\|\partial^{\alpha} \dot{p}\right\|_{L^{2}\left(\Omega_{ \pm}\right)}^{2} \mathrm{~d} \tau \\
& \leq \sum_{ \pm}\left|\int_{0}^{t} \int_{\Omega_{ \pm}} R^{k}(U, \psi) \partial^{\alpha} \partial_{x_{3}}(U, \mu \dot{p}) \mathrm{d} x \mathrm{~d} \tau\right| \\
& \quad+\frac{1}{\varepsilon} t K(t)+\varepsilon\left(M^{0}+t K(t)\right) E_{\mu}(t),
\end{aligned}
$$

where we have used the estimates (111)-(112) for $\dot{p}$. To deal with the first term on the right, we integrate by parts horizontally in space if $\partial^{\alpha}=\partial_{x_{j}} \partial^{\beta}$ or in time if $\partial^{\alpha}=\partial_{t} \partial^{\beta}$ to transfer a derivative to the remainder term, then we use the estimates (118) and (111) or (112).

The proof of (120) is similar to the proof of (118), so we skip the details.

\section{The Linearised Equations}

In this section we introduce a linearisation of the $\mu$-approximate equations which is carefully chosen so that it admits an energy estimate without loss of 
regularity with respect to the coefficients and hence fits nicely into the contractionmapping framework which we intend to use to prove the existence of a fixed point which solves the $\mu$-approximate equations. See Section 4.4 for a brief motivation of the choice of linearisation.

\subsection{Definition of the Linearised Equations}

Definition 32. Let $\bar{T} \in(0, \infty), \bar{f} \in C_{b}^{1}\left((0, \bar{T}) \times \mathbb{R}^{2}\right), \bar{U}_{ \pm}=\left(\bar{p}_{ \pm}, \bar{u}_{ \pm}, \bar{s}_{ \pm}\right) \in$ $C_{b}^{1}\left((0, \bar{T}) \times \Omega_{ \pm}\right)$. Assume that

$$
[\bar{u} \cdot \bar{n}]=0
$$

We say $\left(U_{+}, U_{-}, f\right)$, where $f \in C_{b}^{1}\left((0, T) \times \mathbb{R}^{2}\right), U_{ \pm}=\left(p_{ \pm}, u_{ \pm}, s_{ \pm}\right) \in$ $C_{b}^{1}\left((0, T) \times \Omega_{ \pm}\right)$with $u \in C_{b}^{2}\left(\Omega_{ \pm}\right)$for each $t \in(0, T)$ and $f, u \cdot n \in C_{b}^{2}(\Gamma)$ for each $t \in(0, T)$, is a solution of the linearised equations with lifting operator $L^{a}$ and right hand side $g$ on the time interval $(0, T)$ (where $T \in(0, \bar{T}])$, linearised about the state $\left(\bar{U}_{+}, \bar{U}_{-}, \bar{f}\right)$, if the following holds:

$$
\begin{aligned}
& \left(\left.U_{+}\right|_{t=0},\left.U_{-}\right|_{t=0},\left.f\right|_{t=0}\right)=\left(\left.\bar{U}_{+}\right|_{t=0},\left.\bar{U}_{-}\right|_{t=0},\left.\bar{f}\right|_{t=0}\right) \\
& \rho>0, \quad \rho_{\mu}>0, \quad J^{\psi}:=1+\partial_{x_{3}} \psi>0
\end{aligned}
$$

where

$$
\psi:=L^{a} f
$$

and $p_{ \pm}, u_{ \pm}, s_{ \pm}, f$ satisfy the following system of PDEs.

Firstly,

$$
\partial_{t} f=\bar{u} \cdot \bar{n} \quad \text { on } \quad(0, T) \times \Gamma
$$

In the following equations, we have defined

$$
\overline{u^{\psi}}:=\left(\bar{u}^{\prime}, \frac{1}{J^{\psi}}\left(\bar{u}_{3}-\bar{u}^{\prime} \cdot \nabla^{\prime} \bar{\psi}-\partial_{t} \psi\right)\right) .
$$

Note carefully that the term $\nabla^{\prime} \bar{\psi}$, where $\bar{\psi}:=L^{a} \bar{f}$, instead of $\nabla^{\prime} \psi$ is used so that $\left.\left(\overline{u^{\psi}}\right)_{3}\right|_{\Gamma}=0$.

Next, the following (decoupled) transport equations are to be satisfied in $(0, T) \times \Omega_{ \pm}$.

$$
\begin{array}{r}
\left(\partial_{t}+\overline{u^{\psi}} \cdot \nabla\right) s=0 \\
\left(\partial_{t}+\overline{u^{\psi}} \cdot \nabla\right) p+\bar{\rho} \bar{c}^{2} \nabla^{\psi} \cdot \bar{u}=0 .
\end{array}
$$

We define $\rho$ and $\rho_{\mu}$ from $p$ and $s$ in the obvious manner following previous notation. 
Next, as an intermediate step, we require that there exist $\omega_{ \pm} \in C_{b}^{1}((0, T) \times$ $\Omega_{ \pm}$) which we call the linearised curl, solving the following transport equation in $(0, T) \times \Omega_{ \pm}$.

$\rho_{\mu}\left(\partial_{t}+\overline{u^{\psi}} \cdot \nabla\right) \omega=-\nabla^{\psi} \rho_{\mu} \times\left(\partial_{t}+\overline{u^{\psi}} \cdot \nabla\right) \bar{u}-\rho_{\mu}\left(\varepsilon_{i j k} \partial_{j}^{\psi} \bar{u}_{l} \partial_{l}^{\psi} \bar{u}_{k}\right)+\nabla^{\psi} \times g_{1}$

with

$$
\left.\omega\right|_{t=0}=\left.\left(\nabla^{\bar{\psi}} \times \bar{u}\right)\right|_{t=0} .
$$

Where $\left(\varepsilon_{i j k} \partial_{j}^{\psi} \bar{u}_{l} \partial_{l}^{\psi} \bar{u}_{k}\right)$ denotes the vector with $i$-th component $\varepsilon_{i j k} \partial_{j}^{\psi} \bar{u}_{l} \partial_{l}^{\psi} \bar{u}_{k}$ and we have used summation convention for repeated indices. We call this the linearised curl equation.

Finally, we require that the velocity $u_{ \pm}$solves the following parabolic-type equation:

$$
\begin{aligned}
& \rho_{\mu}\left(\partial_{t}+\overline{u^{\psi}} \cdot \nabla\right) u-\mu \nabla^{\psi}\left(\rho c^{2} \nabla^{\psi} \cdot u\right)+\mu \nabla^{\psi} \times\left(\rho c^{2} \nabla^{\psi} \times u\right) \\
& \quad=-\nabla^{\psi} p+\mu \nabla^{\psi} \times\left(\rho c^{2} \omega\right)+g_{1}
\end{aligned}
$$

in $(0, T) \times \Omega_{ \pm}$and

$$
\begin{gathered}
{[u \cdot n]=0} \\
\mu\left(\Delta^{\prime}-1\right)(u \cdot n)+\mu\left[\rho c^{2} \nabla^{\psi} \cdot u\right]=[p]+\sigma \nabla^{\prime} \cdot \hat{n}-g_{2} \\
n \times\left(\nabla^{\psi} \times u_{ \pm}\right)=n \times \omega_{ \pm}
\end{gathered}
$$

on $(0, T) \times \Gamma$.

\subsection{Definition of the Linearised Energy}

Definition 33. We define the linearised energy $\mathcal{E}_{L}:(0, T] \rightarrow[0, \infty]$ associated to the vector $\left(U_{+}, U_{-}, f\right)$ as being equal to the $\mu$-approximate energy $\mathcal{E}_{\mu}(t)$ associated to the vector $\left(U_{+}, U_{-}, f\right)$ defined in Definition 22, but without the term involving $\nabla^{\psi} \times u$.

We define the energy $\mathcal{E}_{L}(\omega):(0, T] \rightarrow[0, \infty]$ associated with the curl $\omega$ as

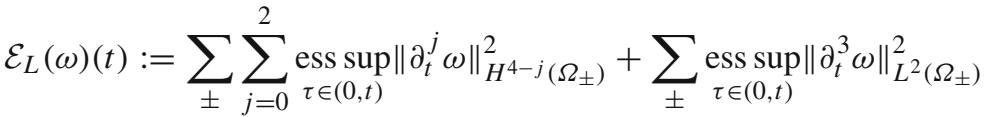

$$
\begin{aligned}
& +\sum_{ \pm} \int_{0}^{t}\left\|\partial_{t}^{3} \omega\right\|_{H^{1}\left(\Omega_{ \pm}\right)}^{2} \mathrm{~d} \tau
\end{aligned}
$$

We also define the slightly lower-order energy $E_{\mu}(\omega):(0, T] \rightarrow[0, \infty]$ associated with $\omega$ as

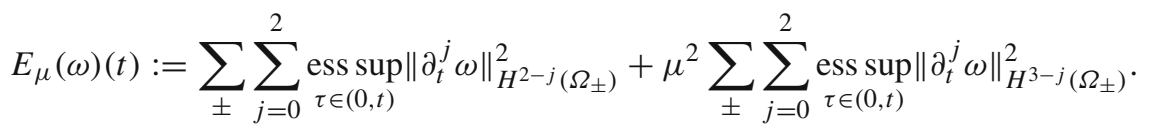




\subsection{Notation for Constants and Functions of the Energy and Initial Data}

Definition 34. For fixed $\mu>0$ it will be convenient to define some generic constants/functions depending on the energy and initial data in a given way which are also allowed to depend on $\mu$.

We will write $F_{\mu}$ for a generic smooth increasing function of its arguments which depends on $\mu$.

We will write $C_{\mu}$ for a generic constant which is independent of the solution, including being independent of the initial data, which depends on $\mu$.

We define $M_{\mu}^{0}$ as generic a constant of the form

$$
M_{\mu}^{0}:=F_{\mu}\left(\mathcal{E}_{\mu}^{0}, \frac{1}{\delta^{0}}, \frac{1}{\delta_{\mu}^{0}}, \frac{1}{\kappa^{0}}, E_{g}(1)\right)
$$

We write $\bar{E}_{\mu}$ to denote the energy $E_{\mu}$ with $\left(U_{+}, U_{-}, f\right)$ replaced by $\left(\bar{U}_{+}, \bar{U}_{-}, \bar{f}\right)$, and we define $\overline{\mathcal{E}}_{L}$ similarly.

We define $K_{\mu}(t)$ as a generic function of $t$ of the form

$$
K_{\mu}(t):=F_{\mu}\left(\bar{E}_{\mu}(t), E_{\mu}(t), E_{\mu}(\omega)(t), M_{\mu}^{0}\right) .
$$

Similarly, we define $\mathcal{K}_{L}(t)$ as a generic function of $t$ of the form

$$
\mathcal{K}_{L}(t):=F_{\mu}\left(\overline{\mathcal{E}}_{L}(t), \mathcal{E}_{L}(t), \mathcal{E}_{L}(\omega)(t), M_{\mu}^{0}\right)
$$

Remark 5. Note that in the following sections, constants and generic functions will be independent of $T$ (for $0<T \leqq 1$ ). In particular, we will only use the Sobolev embedding theorem with respect to space and not with respect to time.

Lemma 15. Suppose $\omega_{ \pm}=\nabla^{\psi} \times u_{ \pm}$. Then

$$
\begin{aligned}
E_{\mu}(\omega)(t) & \leqq F_{\mu}\left(E_{\mu}(t)\right) \\
K_{\mu}(t) & \leqq F_{\mu}\left(\bar{E}_{\mu}(t), E_{\mu}(t), M_{\mu}^{0}\right) \\
\mathcal{E}_{\mu}(t) & \leqq \mathcal{E}_{L}(t)+\mathcal{E}_{L}(\omega)(t) \\
\mathcal{E}_{L}(t) & \leqq \mathcal{E}_{\mu}(t) \\
\mathcal{E}_{L}(\omega)(t) & \leqq F_{\mu}\left(E_{\mu}(t)\right) \mathcal{E}_{\mu}(t) .
\end{aligned}
$$

Proof. The first inequality follows from the definitions of $E_{\mu}(\omega)(t)$ and $E_{\mu}(t)$ and the product rule. The second inequality follows from the first. The third and fourth inequalities are immediate from the definitions of $\mathcal{E}_{\mu}(t), \mathcal{E}_{L}(t)$ and $\mathcal{E}_{L}(\omega)(t)$. The fifth inequality follows from the definitions of $\mathcal{E}_{L}(\omega)(t)$ and $\mathcal{E}_{\mu}(t)$ and the product rule. 
10.4. Statement of the Energy Estimate for the Linearised Equations

Proposition 11. Let $\left(U_{+}^{0}, U_{-}^{0}, f^{0}\right)$ be initial data for the $\mu$-approximate equations with initial energy $\mathcal{E}_{\mu}^{0}<\infty$. Assume that

$$
\inf _{\Omega_{ \pm}} \rho^{0}=: \delta^{0}>0, \quad \inf _{\Omega_{ \pm}} \rho_{\mu}^{0}=: \delta_{\mu}^{0}>0, \quad \inf _{\mathbb{R}^{3}} J^{\psi^{0}}=: \kappa^{0}>0 .
$$

Let $\left(\bar{U}_{+}, \bar{U}_{-}, \bar{f}\right)$ be a background state satisfying the conditions stated in the Definition 32 of the linearised equations above defined on the interval $(0, \bar{T})$. Suppose that in addition the following conditions hold:

$$
\begin{aligned}
\overline{\mathcal{E}}_{\mu}(\bar{T}) & <\infty \\
\left.\partial_{t}^{j}\left(\bar{U}_{+}, \bar{U}_{-}, \bar{f}\right)\right|_{t=0} & =\partial_{0}^{\mu, g, j}\left(U_{+}^{0}, U_{-}^{0}, f^{0}\right) \quad \text { for } 0 \leqq j \leqq 3 .
\end{aligned}
$$

Let $\left(U_{+}, U_{-}, \omega_{+}, \omega_{-}, f\right)$ be a solution of the linearised equations with background state $\left(\bar{U}_{+}, \bar{U}_{-}, \bar{f}\right)$ as defined in the Definition 32 on some time interval $(0, T)$ where $T \in(0, \bar{T})$. Suppose in addition that

$$
\mathcal{E}_{L}(T)+\mathcal{E}_{L}(\omega)(T)<\infty, \quad \rho \geqq \frac{\delta^{0}}{4}, \quad \rho_{\mu} \geqq \frac{\delta_{\mu}^{0}}{4}, \quad J^{\psi} \geqq \frac{1}{4} \kappa^{0}
$$

for $t \in(0, T)$.

Then in fact the energy satisfies the following bound:

$$
\mathcal{E}_{L}(t)+\mathcal{E}_{L}(\omega)(t) \leqq M_{\mu}^{0}+K_{\mu}(t)\left(t+\int_{0}^{t} \overline{\mathcal{E}}_{L}(\tau)+\mathcal{E}_{L}(\tau)+\mathcal{E}_{L}(\omega)(\tau) \mathrm{d} \tau\right)+\frac{1}{4} \overline{\mathcal{E}}_{L}(t)
$$

for $t \in(0, T)$. Moreover, we have

$$
\rho \geqq \delta^{0}-t K_{\mu}(t), \quad \rho_{\mu} \geqq \delta_{\mu}^{0}-t K_{\mu}(t), \quad J^{\psi} \geqq \kappa^{0}-t K_{\mu}(t)
$$

on the time interval $(0, T)$.

Proof. See Section 13.

10.5. Statement of Existence and Uniqueness of Solutions to the Linearised Equations

Proposition 12. Let $\left(U_{+}^{0}, U_{-}^{0}, f^{0}\right)$ be initial data for the $\mu$-approximate equations with initial energy $\mathcal{E}_{\mu}^{0}<\infty$ satisfying the compatibility condition (45) up to order 3 and the condition (46) up to order 2. Assume that

$$
\inf _{\Omega_{ \pm}} \rho^{0}=: \delta^{0}>0, \quad \inf _{\Omega_{ \pm}} \rho_{\mu}^{0}=: \delta_{\mu}^{0}>0, \quad \inf _{\mathbb{R}^{3}} J^{\psi^{0}}=: \kappa^{0}>0 .
$$

Let $\left(\bar{U}_{+}, \bar{U}_{-}, \bar{f}\right)$ be a background state satisfying the conditions stated in the Definition 32 of the linearised equations above defined on the interval $(0, \bar{T})$. Suppose that in addition the following conditions hold:

$$
\overline{\mathcal{E}}_{L}(\bar{T})<\infty
$$




$$
\begin{aligned}
\left.\partial_{t}^{j}\left(\bar{U}_{+}, \bar{U}_{-}, \bar{f}\right)\right|_{t=0}= & \partial_{0}^{\mu, g, j}\left(U_{+}^{0}, U_{-}^{0}, f^{0}\right) \\
& \text { for } 0 \leqq j \leqq 3
\end{aligned}
$$

Then there exists a $T \in(0, \bar{T}]$ and a solution $\left(U_{+}, U_{-}, \omega_{+}, \omega_{-}, f\right)$ of the linearised equations on the time interval $(0, T)$, as defined in Definition 32, satisfying the following additional properties:

$$
\mathcal{E}_{L}(T)+\mathcal{E}_{L}(\omega)(T)<\infty, \quad \rho \geqq \frac{\delta^{0}}{4}, \quad \rho_{\mu} \geqq \frac{\delta_{\mu}^{0}}{4}, \quad J^{\psi} \geqq \frac{1}{4} \kappa^{0}
$$

for all $t \in(0, T)$ and

$$
\begin{aligned}
\left.\partial_{t}^{j}\left(U_{+}, U_{-}, \omega_{+}, \omega_{-}, f\right)\right|_{t=0}= & \partial_{0}^{\mu, g, j}\left(U_{+}^{0}, U_{-}^{0}, \nabla^{\psi^{0}}\right. \\
& \left.\times u_{+}^{0}, \nabla^{\psi^{0}} \times u_{-}^{0}, f^{0}\right) \text { for } 0 \leqq j \leqq 3 .
\end{aligned}
$$

Moreover if $T<\bar{T}$ then one of the following holds as $t \uparrow T$.

$$
\inf _{x \in \Omega_{ \pm}} \rho \rightarrow \frac{\delta^{0}}{4}, \quad \inf _{x \in \Omega_{ \pm}} \rho_{\mu} \rightarrow \frac{\delta_{\mu}^{0}}{4}, \quad \inf _{x \in \mathbb{R}^{3}} J^{\psi} \rightarrow \frac{1}{4} \kappa^{0} .
$$

Also, the solution is unique on the time interval $(0, T)$ amongst solutions with the above properties.

Proof. See Sections 11-12 for the proof.

\subsection{The Curl of the Linearised Velocity Equation}

We record here the result of taking the curl of the linearised velocity equation, although we will only need this when it comes to proving a fixed point of the linearised equations solves the $\mu$-approximate equations.

Lemma 16. Suppose that u satisfies the linearised velocity equation (132). Suppose that in addition to the regularity stated in Definition 32, we have $\partial_{t} \psi, \partial_{t} u, g_{1}, \bar{u} \in$ $H_{\mathrm{loc}}^{1}\left(\Omega_{ \pm}\right), p, s, u, \nabla \psi, \bar{\psi}, \nabla u \in H_{\mathrm{loc}}^{2}\left(\Omega_{ \pm}\right)$. Then $\nabla^{\psi} \times u$ satisfies the following equation.

$$
\begin{aligned}
\rho_{\mu}\left(\partial_{t}+\bar{u}^{\psi} \cdot \nabla\right)\left(\nabla^{\psi} \times u\right)= & -\nabla^{\psi} \rho_{\mu} \times\left(\partial_{t}+\bar{u}^{\psi} \cdot \nabla\right) u-\rho_{\mu}\left(\varepsilon_{i j k} \partial_{j}^{\psi} \bar{u}_{l} \partial_{l}^{\psi} u_{k}\right) \\
& +\nabla^{\psi} \times g_{1} \\
& -\mu \nabla^{\psi} \times\left(\nabla^{\psi} \times\left(\nabla^{\psi} \times u\right)\right)+\mu \nabla^{\psi} \times\left(\nabla^{\psi} \times \omega\right) \\
& -\nabla^{\psi} \times\left(\rho_{\mu} \frac{1}{J \psi} \bar{u}^{\prime} \cdot \nabla^{\prime}(\psi-\bar{\psi}) \partial_{x_{3}} u\right)
\end{aligned}
$$

in $(0, T) \times \Omega_{ \pm}$, where $\left(\varepsilon_{i j k} \partial_{j}^{\psi} \bar{u}_{l} \partial_{l}^{\psi} u_{k}\right)$ denotes the vector with $i$-th component given by $\varepsilon_{i j k} \partial_{j}^{\psi} \bar{u}_{l} \partial_{l}^{\psi} u_{k}$ and we have used summation convention for repeated indices.

Proof. The proof is almost identical to the proof of Lemma 3. 


\section{Solving the Linearised Equations for the Front, Entropy, Pressure and} Curl

In this section we assume we are given the background state $\left(\bar{U}_{+}, \bar{U}_{-}, \bar{f}\right)$ on the time interval $(0, \bar{T})$ and initial data $\left(U_{+}^{0}, U_{-}^{0}, f^{0}\right)$ such that $\left(\bar{U}_{+}, \bar{U}_{-}, \bar{f}\right)$ and $\left(U_{+}^{0}, U_{-}^{0}, f^{0}\right)$ satisfy the hypotheses of Proposition 12 .

We start with the very simple construction of the front $f$ and the lift $\psi$ of the front $f$.

Proposition 13. There exists a $\tilde{T} \in(0, \bar{T}]$ and $f \in L^{\infty}((0, \tilde{T}) \times \Gamma)$ such that

$$
\partial_{t} f=\bar{u} \cdot \bar{n}
$$

on $(0, \tilde{T}) \times \Gamma$ and

$$
\left.\partial_{t}^{j} f\right|_{t=0}=\partial_{0}^{\mu, g, j} f^{0} \text { for } 0 \leqq j \leqq 3 .
$$

Moreover, $f$ has the following regularity:

$$
\begin{gathered}
\partial_{t}^{j} \nabla^{\prime} f \in L^{\infty}\left((0, \tilde{T}) ; H^{4.5-j}(\Gamma)\right) \text { for } 0 \leqq j \leqq 3 \\
\partial_{t}^{j+1} f \in L^{2}\left((0, \tilde{T}) ; H^{5.5-j}(\Gamma)\right) \text { for } 0 \leqq j \leqq 3 \\
\partial_{t}^{j+1} f \in L^{\infty}\left((0, \tilde{T}) ; H^{4-j}(\Gamma)\right) \text { for } 0 \leqq j \leqq 3
\end{gathered}
$$

and such an $f$ is unique.

Also, defining

$$
\psi=L^{a} f
$$

we have

$$
\inf _{x \in \mathbb{R}^{3}} J^{\psi} \geqq \frac{1}{4} \kappa^{0}
$$

for all $t \in(0, \tilde{T})$, and if $\tilde{T}<\bar{T}$, then

$$
\inf _{x \in \mathbb{R}^{3}} J^{\psi} \rightarrow \frac{1}{4} \kappa^{0} \text { as } t \uparrow \tilde{T} \text {. }
$$

Proof. This is clear by direct integration and then setting

$$
\tilde{T}=\sup \left\{t \in(0, \bar{T}): \inf _{\tau \in(0, t)} \inf _{x \in \mathbb{R}^{3}} J^{\psi} \geqq \frac{\kappa^{0}}{4}\right\}
$$

and using continuity. 
Lemma 17. In this lemma functions will be defined either on $\Omega_{+}$or $\Omega_{-}$.

Let $b \in L^{\infty}\left((0, T) ; W^{1, \infty}\left(\Omega_{ \pm} ; \mathbb{R}^{3}\right)\right), h \in L^{2}\left((0, T) ; H^{1}\left(\Omega_{ \pm}\right)\right)$and $w^{0} \in$ $L_{\text {loc }}^{1}\left((0, T) \times \Omega_{ \pm}\right)$with $\nabla w^{0} \in L^{2}\left(\Omega_{ \pm}\right)$. Suppose also that $\left.b_{3}\right|_{\Gamma}=0$. Then there exists a unique solution $w \in L_{\mathrm{loc}}^{1}\left((0, T) \times \Omega_{ \pm}\right)$with $\nabla w, \partial_{t} w \in$ $L^{2}\left((0, T) ; L^{2}\left(\Omega_{ \pm}\right)\right)$of the equation

$$
\left(\partial_{t}+b \cdot \nabla\right) w=h
$$

in $(0, T) \times \Omega_{ \pm}$with

$$
\left.w\right|_{t=0}=w^{0} .
$$

Moreover, iffor some $k \geqq 1, l \geqq 0$ with $k+l \geqq 3$ and all $1 \leqq j \leqq k$,

$$
\begin{aligned}
\partial_{t}^{j} b & \in L^{\infty}\left((0, T) ; H^{k-j+l}\left(\Omega_{ \pm}\right)\right) \\
\partial_{t}^{j-1} h & \in L^{\infty}\left((0, T) ; H^{k-j+l}\left(\Omega_{ \pm}\right)\right) \\
\nabla b & \in L^{\infty}\left((0, T) ; H^{k-1+l}\left(\Omega_{ \pm}\right)\right) \\
\nabla h & \in L^{2}\left((0, T) ; H^{k-1+l}\left(\Omega_{ \pm}\right)\right) \\
\nabla w^{0} & \in H^{k-1+l}\left(\mathbb{R}^{d}\right),
\end{aligned}
$$

then

$$
\begin{aligned}
\partial_{t}^{j} w & \in L^{\infty}\left((0, T) ; H^{k-j+l}\left(\Omega_{ \pm}\right)\right) \\
\nabla w & \in L^{\infty}\left((0, T) ; H^{k-1+l}\left(\Omega_{ \pm}\right)\right)
\end{aligned}
$$

for all $1 \leqq j \leqq k$

Proof. Uniqueness follows easily by applying a standard energy estimate (which is easily derived because $\left.b_{3}\right|_{\Gamma}=0$ ) to the difference of two solutions and using Gronwall's lemma.

To prove the existence we simply apply the Sobolev extension operator Ext $\Omega_{\Omega_{+}}$in space to extend $b, h$ and $w^{0}$ to be defined on $\mathbb{R}^{3}$. Then we are left with a completely standard scalar linear transport equation on the whole space which we may solve to obtain $w \in L_{\text {loc }}^{1}\left((0, T) \times \mathbb{R}^{3}\right)$ with $\nabla w, \partial_{t} w \in L^{2}\left((0, T) ; L^{2}\left(\mathbb{R}^{3}\right)\right)$ satisfying the equation in $(0, T) \times \mathbb{R}^{3}$. Then $\left.w\right|_{\Omega_{ \pm}}$satisfies the equation in $\Omega_{ \pm}$and is the unique solution by the above.

Also, the regularity of $\left.w\right|_{\Omega_{ \pm}}$follows from the regularity of $w$ on the whole space given by the standard theory of transport equations and the Sobolev embedding theorem.

Proposition 14. Let $\tilde{T}, f$ and $\psi$ be given by Proposition 13. Then there exist $T \in(0, \tilde{T})$ and $s, p, \omega \in L^{\infty}\left((0, T) \times \Omega_{ \pm}\right)$satisfying the linearised equations for the entropy, pressure and curl, (128)-(130), with

$$
\begin{aligned}
\left.\partial_{t}^{j} s\right|_{t=0} & =\partial_{0}^{\mu, g, j} s^{0} \text { for } 0 \leqq j \leqq 3 \\
\left.\partial_{t}^{j} p\right|_{t=0} & =\partial_{0}^{\mu, g, j} p^{0} \text { for } 0 \leqq j \leqq 3
\end{aligned}
$$




$$
\left.\partial_{t}^{j} \omega\right|_{t=0}=\partial_{0}^{\mu, g, j}\left(\nabla^{\psi^{0}} \times u^{0}\right) \text { for } 0 \leqq j \leqq 3 .
$$

Moreover, they have the following regularity:

$$
\begin{aligned}
\partial_{t}^{j} s, \partial_{t}^{j} p, \partial_{t}^{j} \omega & \in L^{\infty}\left((0, T) ; H^{4-j}\left(\Omega_{ \pm}\right)\right) \text {for } 1 \leqq j \leqq 3 \\
\nabla s, \nabla p, \nabla \omega & \in L^{\infty}\left((0, T) ; H^{3}\left(\Omega_{ \pm}\right)\right) \\
\partial_{t}^{4} s, \partial_{t}^{4} p & \in L^{\infty}\left((0, T) ; L^{2}\left(\Omega_{ \pm}\right)\right) \\
\partial_{t}^{4} \omega & \in L^{2}\left((0, T) ; L^{2}\left(\Omega_{ \pm}\right)\right),
\end{aligned}
$$

and such $s, p, \omega$ are unique. In addition,

$$
\begin{gathered}
\rho \geqq \frac{\delta^{0}}{4} \quad \text { in } \quad(0, T) \times \Omega_{ \pm} \\
\rho_{\mu} \geqq \frac{\delta_{\mu}^{0}}{4} \quad \text { in } \quad(0, T) \times \Omega_{ \pm},
\end{gathered}
$$

and if $T<\tilde{T}$, then one of the following holds:

$$
\begin{gathered}
\inf _{x \in \Omega_{ \pm}} \rho \rightarrow \frac{\delta^{0}}{4} \text { as } t \uparrow T \\
\inf _{x \in \Omega_{ \pm}} \rho_{\mu} \rightarrow \frac{\delta_{\mu}^{0}}{4} \text { as } t \uparrow T .
\end{gathered}
$$

Proof. We start by solving the linearised entropy equation (128). Apply Lemma 17 with $b=\overline{u^{\psi}}, h=0$ and $w^{0}=s^{0}$ to obtain a unique solution $s \in L_{\mathrm{loc}}^{1}\left((0, \tilde{T}) \times \Omega_{ \pm}\right)$ with $\nabla s \in L^{2}\left((0, \tilde{T}) ; L^{2}\left(\Omega_{ \pm}\right)\right)$and $\partial_{t} s \in L^{2}\left((0, \tilde{T}) ; L^{2}\left(\Omega_{ \pm}\right)\right)$to the equation with $s(0)=s^{0}$. The claimed regularity easily follows from the regularity of $\bar{u}, \bar{\psi}, \psi$ and $s^{0}$.

To see that the initial time derivatives match, we use the fact that the background state satisfies (136) together with $s(0)=s^{0}$, which show that the linearised equation for $s$ is the same as the $\mu$-approximate equation for $s$ when restricted to $t=0$, which shows that (138) holds for $j=1$. We then proceed by induction.

We apply exactly the same reasoning for the pressure. We then set $T=\sup \{t \in$ $\left.(0, \tilde{T}): \inf _{\tau \in(0, t)} \inf _{x \in \Omega_{ \pm}} \rho \geqq \frac{\delta^{0}}{4}, \inf _{\tau \in(0, t)} \inf _{x \in \Omega_{ \pm}} \rho_{\mu} \geqq \frac{\delta_{\mu}^{0}}{4}\right\}$ to provide the bounds on the density, which we recall is a function of $s$ and $p$. We then treat the curl in the same way as the entropy and pressure, except that due to the fact that we only have $\partial_{t}^{4} u \in L^{2}\left((0, T) ; L^{2}\left(\Omega_{ \pm}\right)\right)$, we obtain only $\partial_{t}^{4} \omega \in L^{2}\left((0, T) ; L^{2}\left(\Omega_{ \pm}\right)\right)$.

\section{Solving the Linearised Parabolic-Type Equation for the Velocity}

The basic approach to solving the linearised equation for the velocity uses a weak form of the equation and a Galerkin approximation with energy estimates for the Galerkin approximation providing additional regularity—see eg EvANs [14] for a simple version of this method. 
In this section we assume we are given the background state $\left(\bar{U}_{+}, \bar{U}_{-}, \bar{f}\right)$ on the time interval $(0, \bar{T})$ and initial data $\left(U_{+}^{0}, U_{-}^{0}, f^{0}\right)$ which together satisfy the hypotheses of Proposition 12. Given this initial data and background state, we assume that $f$ and $\psi$ are given by Proposition 13, $s, p, T, \rho, \rho_{\mu}$ and $\omega$ are given by Proposition 14 .

\subsection{Notation for Constants Depending on the Background State and the Solutions to Preceding Equations}

Definition 35. It will be useful to define constants depending on $\nabla^{\prime} f, \rho_{\mu}, \rho c^{2}$ which we will use as a bound on coefficients of the equations. For $0 \leqq j \leqq 1$, we define $C_{\infty, j}>0$ as a generic constant which is bounded above as follows:

$$
\begin{aligned}
C_{\infty, j}(t) \leqq F_{\mu}( & \sum_{ \pm} \sum_{k=0}^{j} \underset{\tau \in(0, t)}{\operatorname{ess} \sup \left\|\partial_{t}^{k}\left(\rho c^{2}\right)\right\|_{W^{j-k, \infty}\left(\Omega_{ \pm}\right)}^{2},} \\
& \sum_{ \pm} \sum_{k=0}^{j} \underset{\tau \in(0, t)}{\operatorname{ess} \sup \left\|\partial_{t}^{k}\left(\rho_{\mu}, \partial_{t} \rho_{\mu}\right)\right\|_{W^{j-k, \infty}\left(\Omega_{ \pm}\right)}^{2},} \\
& \left.\sum_{k=0}^{j+1} \operatorname{ess} \sup \left\|\partial_{t}^{k} \nabla^{\prime} f\right\|_{H^{2.5+j-k}(\Gamma)}^{2}, \frac{1}{\delta^{0}}, \frac{1}{\delta_{\mu}^{0}}, \frac{1}{\kappa^{0}}\right),
\end{aligned}
$$

for some smooth increasing function $F_{\mu}$ depending on $\mu$.

\subsection{Statement of Existence and Uniqueness of Solutions to the Parabolic-Type} Linearised Equation for the Velocity

Proposition 15. Let $f$ and $\psi$ be given by Proposition 13, s, p, T, $\rho, \rho_{\mu}$ and $\omega$ be given by Proposition 14. Then there exists $u \in L^{\infty}\left((0, T) \times \Omega_{ \pm}\right)$such that

$$
\begin{aligned}
& \rho_{\mu}\left(\partial_{t}+\overline{u^{\psi}} \cdot \nabla\right) u-\mu \nabla^{\psi}\left(\rho c^{2} \nabla^{\psi} \cdot u\right)+\mu \nabla^{\psi} \times\left(\rho c^{2} \nabla^{\psi} \times u\right) \\
& \quad=-\nabla^{\psi} p+\mu \nabla^{\psi} \times\left(\rho c^{2} \omega\right)+g_{1}
\end{aligned}
$$

in $(0, T) \times \Omega_{ \pm}$and

$$
\begin{aligned}
{[u \cdot n] } & =0 \\
\mu\left(\Delta^{\prime}-1\right)(u \cdot n)+\mu\left[\rho c^{2} \nabla^{\psi} \cdot u\right] & =[p]+\sigma \nabla^{\prime} \cdot \hat{n}-g_{2} \\
n \times\left(\nabla^{\psi} \times u_{ \pm}\right) & =n \times \omega_{ \pm}
\end{aligned}
$$

on $(0, T) \times \Gamma$ and

$$
\left.\partial_{t}^{j} u\right|_{t=0}=\partial_{0}^{\mu, g, j} u^{0} \text { for } 0 \leqq j \leqq 3
$$

Moreover

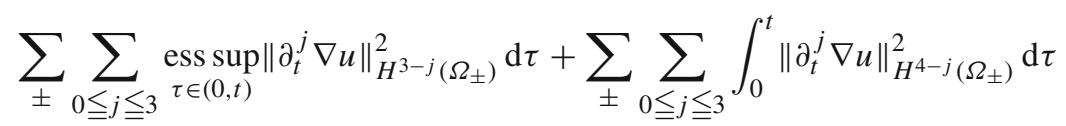




$$
\begin{aligned}
& +\sum_{ \pm} \int_{0}^{t}\left\|\partial_{t}^{4} u\right\|_{L^{2}\left(\Omega_{ \pm}\right)}^{2} \mathrm{~d} \tau+\sum_{j=0}^{3} \underset{\tau \in(0, t)}{\operatorname{ess} \sup \left\|\partial_{t}^{j}(u \cdot n)\right\|_{H^{4-j}(\Gamma)}^{2}} \\
& +\sum_{j=0}^{3} \int_{0}^{t}\left\|\partial_{t}^{j}(u \cdot n)\right\|_{H^{5.5-j}(\Gamma)}^{2} \mathrm{~d} \tau<\infty
\end{aligned}
$$

Proof. See the remainder of this chapter for the proof.

\subsection{Rotating the Velocity to Fix the Normal Jump Condition}

Definition 36. For the purposes of this section we will extend $\nabla^{\prime} f$ from $\Gamma$ to $\nabla^{\prime} f$ defined on $\mathbb{R}^{3}$ via a linear lifting operator which gains half a derivative, so that

$$
\left\|\nabla^{\prime} f\right\|_{H^{l+1}\left(\mathbb{R}^{3}\right)} \leqq C\left\|\nabla^{\prime} f\right\|_{H^{l+0.5}(\Gamma)}
$$

for $l \geqq 0$. See Lemma 2 for a construction of such a lifting operator.

We define the matrix $N$ depending on $\nabla^{\prime} f$ as a scalar multiple of an orthogonal matrix, with columns as follows:

$$
N=\frac{1}{|\underset{\sim}{n}|}\left(\hat{\tau}^{1} \hat{\tau}^{2} \hat{\hat{n}}\right),
$$

where

$$
\begin{aligned}
& \underline{\sim}=\left(-\nabla^{\prime} f, 1\right) \\
& \tau^{1}=\left(1,0, \partial_{x_{1}} f\right) \\
& \tau^{2}=\underset{\sim}{n} \times{\underset{\sim}{\tau}}^{1}=\left(-\underline{\partial}_{x_{1}} f \partial_{x_{2}} f, 1+\left(\partial_{x_{1}} f\right)^{2}, \partial_{x_{2} f}\right)
\end{aligned}
$$

and

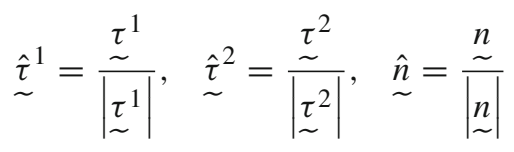

are orthogonal each other, so that $\left(\hat{\tau}^{1}, \hat{\tau}^{2}, \underline{\hat{n}}\right)$ form a right-handed orthonormal basis of $\mathbb{R}^{3}$ for each $(t, x) \in(0, T) \times \mathbb{R}^{3}$.

We write $N^{T}$ for the transpose of $N$ and $N^{-1}$ for its inverse. Note that by orthogonality, we have

$$
N^{-1}=|\underset{\sim}{n}|^{2} N^{T}
$$

Note that orthogonality also implies that, for any vector $w$,

$$
N w=\frac{1}{|\underset{\sim}{n}|}\left(w_{\sim}^{\hat{\tau}^{1}}+w_{2} \hat{\tau}^{2}+w_{3} \hat{\sim}\right)
$$




$$
\begin{aligned}
& (N w) \cdot \underset{\sim}{n}=w_{3} \\
& \left(N^{-1} w\right)_{3}=w \cdot \underset{\sim}{n} .
\end{aligned}
$$

We also write $\bar{N}$ to denote the matrix given by replacing $f$ with $\bar{f}$ in the definition of $N$.

Definition 37. We define the rotated initial velocity

$$
\stackrel{\sim}{u}^{0}:=\left.N^{-1}\right|_{t=0} u^{0} .
$$

The initial time derivatives act on $\stackrel{\overbrace{}}{u}^{0}$ in the obvious way. For $0 \leqq j \leqq 3$,

$$
\partial_{0}^{\mu, g, j} \stackrel{\sim}{u}^{0}:=\left.\partial_{t}^{j}\left(N^{-1}(t) u(t)\right)\right|_{t=0},
$$

where on the right hand side we take the formal derivative with respect to $t$ then replace $\left.\partial_{t}^{l} u\right|_{t=0}$ with $\partial_{0}^{\mu, g, l} u^{0}$ for $0 \leqq l \leqq j$. Note that we have $\left.\partial_{t}^{j} f\right|_{t=0}=\partial_{0}^{\mu, g, j} f^{0}$ for $0 \leqq j \leqq 3$, so if we write $N^{0}$ for the matrix defined by replacing $\nabla^{\prime} f$ with $\nabla^{\prime} f^{0}$ in the definition of $N$, then we may write $\left.\partial_{t}^{j} N\right|_{t=0}=\partial_{0}^{\mu, g, j} N^{0}$.

Definition 38. We say that the rotated velocity function $\stackrel{\overbrace{}}{u}_{ \pm} \in C_{b}^{2}\left((0, T) \times \Omega_{ \pm}\right)$ with $\stackrel{\mathcal{u}}{3}_{3} \in C_{b}^{2}((0, T) \times \Gamma)$ is a solution of the rotated linearised equations for the velocity if $\stackrel{\vec{u}}{u}(0)=\stackrel{\overbrace{}}{u}^{0}$ and $\stackrel{\leftrightarrow}{u}$ satisfies the following equations:

$$
\begin{aligned}
& \rho_{\mu} \frac{1}{|\underset{\sim}{n}|^{2}} \partial_{t} \stackrel{\curvearrowright}{u}-\mu N^{T} \nabla^{\psi}\left(\rho c^{2} \nabla^{\psi} \cdot(N \stackrel{\tilde{u}}{)})+\mu N^{T} \nabla^{\psi} \times\left(\rho c^{2} \nabla^{\psi} \times(N \stackrel{\widetilde{u}}{)})\right)\right.
\end{aligned}
$$

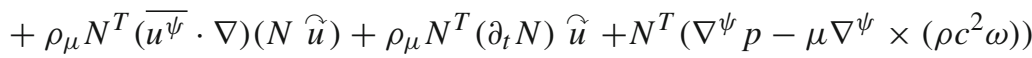

$$
\begin{aligned}
& =N^{T} g_{1}
\end{aligned}
$$

in $(0, T) \times \Omega_{ \pm}$and

$$
\begin{aligned}
{\left[\widetilde{u}_{3}\right] } & =0 \\
\mu\left(\Delta^{\prime}-1\right) \stackrel{\widehat{u}}{3}+_{3}+\mu\left[\rho c^{2} \nabla^{\psi} \cdot(N \tilde{\tilde{u}})\right] & =[p]+\sigma \nabla^{\prime} \cdot \hat{n}-g_{2} \\
n \times\left(\nabla^{\psi} \times\left(N \widetilde{\tilde{u}}_{ \pm}\right)\right) & =n \times \omega_{ \pm}
\end{aligned}
$$

on $(0, T) \times \Gamma$.

Proposition 16. Suppose $\tilde{u}$ is a solution of the rotated linearised equations for the velocity as defined in Definition 38 satisfying

$$
\begin{aligned}
& \left.\partial_{t}^{j} \stackrel{\curvearrowright}{u}\right|_{t=0}=\partial_{0}^{\mu, g, j} \stackrel{\overbrace{}}{u} \quad \text { for } 0 \leqq j \leqq 3
\end{aligned}
$$

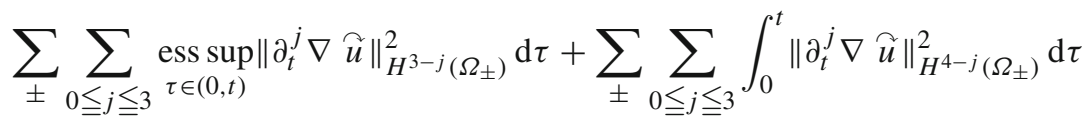

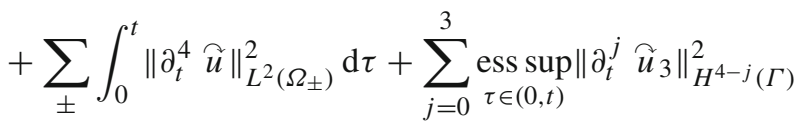




$$
+\sum_{j=0}^{3} \int_{0}^{t}\left\|\partial_{t}^{j} \stackrel{\curvearrowright}{u}_{3}\right\|_{H^{5.5-j}(\Gamma)}^{2} \mathrm{~d} \tau
$$

$<\infty$.

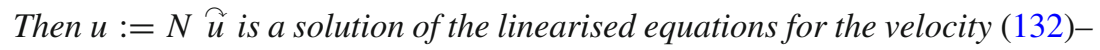
(135) with

$$
\begin{aligned}
& \left.\partial_{t}^{j} u\right|_{t=0}=\partial_{0}^{\mu, g, j} u^{0} \text { for } 0 \leqq j \leqq 3 \\
& \sum_{ \pm} \sum_{0 \leqq j \leqq 3} \operatorname{ess~sup}_{\tau \in(0, t)} \partial_{t}^{j} \nabla u\left\|_{H^{3-j}\left(\Omega_{ \pm}\right)}^{2} \mathrm{~d} \tau+\sum_{ \pm} \sum_{0 \leqq j \leqq 3} \int_{0}^{t}\right\| \partial_{t}^{j} \nabla u \|_{H^{4-j}\left(\Omega_{ \pm}\right)}^{2} \mathrm{~d} \tau \\
& \quad+\sum_{ \pm} \int_{0}^{t}\left\|\partial_{t}^{4} u\right\|_{L^{2}\left(\Omega_{ \pm}\right)}^{2} \mathrm{~d} \tau+\sum_{j=0}^{3} \operatorname{ess} \sup \left\|\partial_{t}^{j}(u \cdot n)\right\|_{H^{4-j}(\Gamma)}^{2} \\
& \quad+\sum_{j=0}^{3} \int_{0}^{t}\left\|\partial_{t}^{j}(u \cdot n)\right\|_{H^{5.5-j}(\Gamma)}^{2} \mathrm{~d} \tau<\infty .
\end{aligned}
$$

Proof. This is straightforward to check by setting $\overparen{u}=N^{-1} u$ in the equations (141)-(144). Note that the energy estimate requires the fact that

$$
\int_{0}^{t}\|N\|_{H^{5}\left(\Omega_{ \pm}\right)}^{2} \mathrm{~d} \tau \leqq C \int_{0}^{t}\left\|\nabla^{\prime} f\right\|_{H^{4.5}(\Gamma)}^{2} \mathrm{~d} \tau<\infty,
$$

due to the lifting property.

\subsection{The Spaces for a Weak Form of the Equations}

Definition 39. For $r \in[1, \infty]$, we define the domains $\Omega_{r \pm}$ and interface $\Gamma_{r}$ as follows:

$$
\begin{aligned}
\Omega_{r \pm} & :=\Omega_{ \pm} \cap B_{r}(0) \\
\Gamma_{r} & :=\Gamma \cap B_{r}(0),
\end{aligned}
$$

where $B_{r}(0)$ denotes the open ball of radius $r$ about 0 , and is equal to $\mathbb{R}^{3}$ in the case $r=\infty$. For $r<\infty$ we refer to these as the bounded domains.

Definition 40. For $r \in[1, \infty]$, we define the Hilbert spaces $B_{r}^{0}$ and $B_{r}^{1}$ as follows.

$$
B_{r}^{0}:=\left\{v=\left(v_{+}, v_{-}\right) \in L^{2}\left(\Omega_{r+} ; \mathbb{R}^{3}\right) \times L^{2}\left(\Omega_{r-} ; \mathbb{R}^{3}\right)\right\} .
$$

For each $t \in[0, T]$ (at the end points we use uniform continuity for the following definition) we define the inner product $(\cdot, \cdot)_{B_{r}^{0}(t)}$ on $B_{r}^{0}$ as follows, for $v, w \in B_{r}^{0}$.

$$
(v, w)_{B_{r}^{0}(t)}=\sum_{ \pm}\left(\rho_{\mu}(t) \frac{1}{|\underset{\sim}{n}(t)|^{2}} v, w\right)_{L^{2}\left(\Omega_{r \pm}\right)} .
$$


We define the inner product $(\cdot, \cdot)_{B_{r}^{0}}$ as

$$
(v, w)_{B_{r}^{0}}=(v, w)_{B_{r}^{0}(0)},
$$

which is independent of time. We will use this inner product on $B_{r}^{0}$ when applying any results from functional analysis. The other inner products are defined for notational convenience. Note that for all $t \in[0, T]$ the inner product $(\cdot, \cdot)_{B_{r}^{0}(t)}$ is equivalent to the standard inner product on $L^{2}\left(\Omega_{r+}\right) \times L^{2}\left(\Omega_{r-}\right)$ since there exists a constant $C_{\infty, 0}>0$ such that

$$
\begin{gathered}
\frac{1}{C_{\infty, 0}} \leqq \rho_{\mu \pm}(t, x) \leqq C_{\infty, 0} \\
\frac{1}{C_{\infty, 0}} \leqq|\underset{\sim}{n}(t, x)| \leqq C_{\infty, 0}
\end{gathered}
$$

for all $(t, x)$.

We define

$$
\begin{gathered}
B_{r}^{1}:=\left\{v=\left(v_{+}, v_{-}\right) \in H^{1}\left(\Omega_{r+} ; \mathbb{R}^{3}\right) \times H^{1}\left(\Omega_{r-} ; \mathbb{R}^{3}\right):\right. \\
\left.\left[v_{3}\right]=0,\left.v_{3}\right|_{\Gamma_{r}} \in H^{1}\left(\Gamma_{r}\right),\left.v\right|_{|x|=r}=0\right\} .
\end{gathered}
$$

Note that by $\left.v\right|_{|x|=r}=0$ we mean that the $H^{1}\left(\Omega_{r \pm}\right)$-trace of $v$ on $\{|x|=r\} \cap \Omega_{ \pm}$ is zero and the $H^{1}\left(\Gamma_{r}\right)$-trace of $v_{3}$ on $\{|x|=r\} \cap \Gamma$ is zero, and this condition should be dropped entirely in the case $r=\infty$. For each $t \in[0, T]$ (at the end points we use uniform continuity for the following definition) we define the semi-norminducing-inner product $\{\cdot, \cdot\}_{B_{r}^{1}(t)}$ on $B_{r}^{1}$ as follows, for $v, w \in B_{r}^{1}$.

$$
\begin{aligned}
\{v, w\}_{B_{r}^{1}(t)}= & \sum_{ \pm}\left(\rho(t) c^{2}(t) \nabla^{\psi(t)} \cdot(N(t) v), \nabla^{\psi(t)} \cdot(N(t) w)\right)_{L^{2}\left(\Omega_{r \pm}\right)} \\
& +\sum_{ \pm}\left(\rho(t) c^{2}(t) \nabla^{\psi(t)} \times(N(t) v), \nabla^{\psi(t)} \times(N(t) w)\right)_{L^{2}\left(\Omega_{r \pm}\right)} \\
& +\left(\nabla^{\prime} v_{3}, \nabla^{\prime} w_{3}\right)_{L^{2}\left(\Gamma_{r}\right)}+\left(v_{3}, w_{3}\right)_{L^{2}\left(\Gamma_{r}\right)} .
\end{aligned}
$$

We define the semi-norm-inducing-inner product $\{\cdot, \cdot\}_{B_{r}^{1}}$ as

$$
\{v, w\}_{B_{r}^{1}}=\{v, w\}_{B_{r}^{1}(0)},
$$

which is independent of time.

We will write the induced semi-norm as

$$
|v|_{B_{r}^{1}(t)}^{2}:=\{v, v\}_{B_{r}^{1}(t)} .
$$

We then define the time-dependent full inner product and induced norm on $B_{r}^{1}$ as

$$
\begin{aligned}
(v, w)_{B_{r}^{1}(t)} & =(v, w)_{B_{r}^{0}(t)}+\{v, w\}_{B_{r}^{1}(t)} \\
\|v\|_{B_{r}^{1}(t)}^{2} & =(v, v)_{B_{r}^{0}(t)}+\{v, v\}_{B_{r}^{1}(t)}
\end{aligned}
$$


and the time-independent full inner product

$$
(v, w)_{B_{r}^{1}}=(v, w)_{B_{r}^{0}}+\{v, w\}_{B_{r}^{1}} .
$$

We will use this inner product on $B_{r}^{1}$ when applying any results from functional analysis. The other inner products are defined for notational convenience.

We will sometimes write $B^{0}$ and $B^{1}$ in the case $r=\infty$.

Proposition 17. The map $\Phi: B_{r}^{1} \rightarrow \Phi\left(B_{r}^{1}\right) \subset H^{1}\left(\Omega_{r+} ; \mathbb{R}^{3}\right) \times H^{1}\left(\Omega_{r-} ; \mathbb{R}^{3}\right) \times$ $H^{1}\left(\Gamma_{r}\right)$, given by

$$
v \mapsto\left(v_{+}, v_{-},\left.v_{3}\right|_{\Gamma}\right),
$$

is an isomorphism between Hilbert spaces, where the target space is equipped with the obvious product inner product, and there exists $C_{\infty, 0}>0$ independent of time such that

$$
\begin{aligned}
& \frac{\|v\|_{B_{r}^{1}(t)}^{2}}{C_{\infty, 0}} \leqq\|\Phi(v)\|^{2}:=\left\|v_{+}\right\|_{H^{1}\left(\Omega_{r+}\right)}^{2}+\left\|v_{-}\right\|_{H^{1}\left(\Omega_{r-}\right)}^{2}+\left\|v_{3}\right\|_{H^{1}\left(\Gamma_{r}\right)}^{2} \\
& \quad \leqq C_{\infty, 0}\|v\|_{B_{r}^{1}(t)}^{2}
\end{aligned}
$$

for all $t \in[0, T]$.

Proof. The proof is straightforward using the orthogonality of $|\underline{n}| N(t)$ and the Hodge decomposition estimate given in Proposition 36 applied to $N(t) v$.

Corollary 2. For $r<\infty, B_{r}^{1}$ is compactly embedded in $B_{r}^{0}$ (with norms $\|\cdot\|_{B_{r}^{1}(t)}$ and $\|\cdot\|_{B_{r}^{0}(t)}$ respectively).

Proof. Using the above result and the standard Sobolev compact embedding theorem, we have $B_{r}^{1} \subset H^{1}\left(\Omega_{r+} ; \mathbb{R}^{3}\right) \times H^{1}\left(\Omega_{r-} ; \mathbb{R}^{3}\right) \subset \subset L^{2}\left(\Omega_{r+} ; \mathbb{R}^{3}\right) \times$ $L^{2}\left(\Omega_{r-} ; \mathbb{R}^{3}\right)$, where the symbol $\subset \subset$ means compactly contained. Now we have already shown that $L^{2}\left(\Omega_{r+} ; \mathbb{R}^{3}\right) \times L^{2}\left(\Omega_{r-} ; \mathbb{R}^{3}\right)$ is isomorphic to $B_{r}^{0}$ with equivalent norms, and this proves the result.

Definition 41. For $r \in[1, \infty]$, we define the space $X_{r}$ as follows:

$$
X_{r}=\left\{v \in L^{\infty}\left((0, T) ; B_{r}^{1}\right): \partial_{t} v \in L^{2}\left((0, T) ; B_{r}^{0}\right)\right\}
$$

We will sometimes write $X$ for the case $r=\infty$.

Note that if $v \in X$ then $v \in C\left([0, T] ; B_{r}^{0}\right)$, since $\partial_{t} v \in L^{2}\left((0, T) ; B_{r}^{0}\right)$. 


\subsection{Construction of a Galerkin Basis}

Lemma 18. Let $m \geqq 1$ be an integer, and let $z_{1}, \ldots, z_{m} \in B_{r}^{0}$ be orthonormal with respect to the inner product $(\cdot, \cdot)_{B_{r}^{0}}$. Define the matrix $M(t)$ depending on $t \in(0, T)$ by

$$
(M(t))_{k l}=\left(z_{k}, z_{l}\right)_{B_{r}^{0}(t)} .
$$

Then $M(t)$ is uniformly positive definite on $(0, T)$, ie there exists a constant $C_{\infty, 0}>$ 0 independent of $t$ such that

$$
b^{T} M(t) b \geqq \frac{1}{C_{\infty, 0}}|b|^{2}
$$

for all $b \in \mathbb{R}^{m}$, and hence $M(t)$, is invertible with

$$
\left|M(t)^{-1}\right| \leqq C_{\infty, 0}
$$

for some $C_{\infty}>0$ independent of $t$. Hence, since $M \in W^{1, \infty}(0, T)$ with

$$
\left|\partial_{t} M\right| \leqq C_{\infty, 0},
$$

we have that $M^{-1} \in W^{1, \infty}(0, T)$ with

$$
\left|\partial_{t} M^{-1}\right| \leqq C_{\infty, 0} .
$$

Proof. The proof is straightforward by considering $b^{T} M(t) b$ for $b \in \mathbb{R}^{m}$.

Lemma 19. For $r<\infty$, there exists a sequence of functions $\left(z_{k}\right)_{k=1}^{\infty} \subset B_{r}^{1}$ which form an orthogonal Hilbert space basis for $B_{r}^{1}$ (with norm $\|\cdot\|_{B_{r}^{1}}$ ) and an orthonormal Hilbert space basis for $B_{r}^{0}$ (with norm $\|\cdot\|_{B_{r}^{0}}$ ). We call these the Galerkin basis functions. Moreover, the functions $z_{k}$ satisfy the following eigenvalue equation:

$$
\left(z_{k}, w\right)_{B_{r}^{1}}=\lambda_{k}\left(z_{k}, w\right)_{B_{r}^{0}} \text { for all } w \in B_{r}^{1},
$$

where $\lambda_{k}>0$ for each $k$.

Proof. We define the bounded linear operator $S: B_{r}^{0} \rightarrow B_{r}^{0}$ as follows:

$$
S f=u
$$

where $u \in B_{r}^{1} \subset B_{r}^{0}$ is the unique solution to the equation

$$
(u, w)_{B_{r}^{1}}=(f, w)_{B_{r}^{0}} \text { for all } w \in B_{r}^{1} .
$$

It is a standard calculation (usually applied to the Laplacian operator) to show that $S$ is a well-defined (using the Riesz representation theorem) symmetric positive definite operator and it is compact since $S f \in B_{r}^{1} \subset \subset B_{r}^{0}$. We then use the spectral decomposition theorem for compact self-adjoint operators to obtain an orthonormal basis $\left(z_{k}\right)_{k=1}^{\infty}$ of $B_{r}^{0}$ that are eigenfunctions of $S$ with eigenvalues $\lambda_{k}^{-1}>0$. The eigenvalue property can also by used to show that $\left(z_{k}\right)_{k=1}^{\infty}$ is an orthogonal basis of $B_{r}^{1}$. 
12.6. Existence, Uniqueness and Regularity Theory for Weak Equations of the Appropriate Form

Notation. If $v$ is a vector, we will write $(v, \nabla v)$ as a matrix containing all the entries of $v$ and all the derivatives $\partial_{x_{j}} v_{i}$ for $0 \leqq i, j \leqq 3$. We write just $\nabla v$ for the matrix with entries $\partial_{x_{j}} v_{i}$ for $0 \leqq i, j \leqq 3$.

For simplicity, we will denote a linear function of $v, \nabla v$ or $(v, \nabla v)$ whose output may be a scalar, a vector, or a matrix, by an expression of the form

$$
\begin{aligned}
& A v \\
& A \nabla v \\
& \text { or } A(v, \nabla v)
\end{aligned}
$$

respectively, where $A$ is a tensor of the appropriate rank.

We will also be using $G$ to denote a generic scalar, vector or matrix as appropriate, but note in particular that if $G$ is a matrix and $(\cdot, \cdot)$ denotes an inner product then

$$
(G, \nabla v)=\sum_{i, j=1}^{3} G_{i j} \partial_{x_{j}} v_{i}
$$

Also, if $G$ is a matrix then $G_{j}$ will denote the $j$-th column of $G$ and $\nabla \cdot$ acts on the rows of $G$.

We may write $C_{G}$ for a finite constant which depends on $G$ (or, later on, $G^{i}$ for $1 \leqq i \leqq 4)$.

Definition 42. We will sometimes find it convenient to write

$$
\begin{aligned}
\left(\partial_{t} v, w\right)_{B_{r}^{0}(t)} & =\sum_{ \pm}\left(A^{0} \partial_{t} v, w\right)_{L^{2}\left(\Omega_{r \pm}\right)} \\
\{v, w\}_{B_{r}^{1}(t)} & =\sum_{ \pm}\left(A^{1}(v, \nabla v),(w, \nabla w)\right)_{L^{2}\left(\Omega_{r \pm}\right)}+\left(v_{3}, w_{3}\right)_{H^{1}\left(\Gamma_{r}\right)}
\end{aligned}
$$

for a scalar function $A^{0}$ and a tensor of appropriate rank $A^{1}$.

We also define the tensor $A^{2}$ such that

$$
\begin{aligned}
A^{2}(v, \nabla v)= & \mu N^{T} \partial_{x_{3}}\left(\frac{\left(-\nabla^{\prime} \psi, 1\right)}{J^{\psi}}\right) \rho c^{2} \nabla^{\psi} \cdot(N v) \\
& \left.-\mu N^{T} \partial_{x_{3}}\left(\frac{\left(-\nabla^{\prime} \psi, 1\right)}{J^{\psi}}\right) \times\left(\nabla^{\psi} \times(N v)\right)+\rho_{\mu} N^{T} \overline{\left(u^{\psi}\right.} \cdot \nabla\right)(N v) \\
& +\rho_{\mu} N^{T}\left(\partial_{t} N\right) v
\end{aligned}
$$

We will write $C_{A}$ for a finite constant that depends $A^{i}$ for $0 \leqq i \leqq 2$. 
Proposition 18. Let $r \in[1, \infty]$ and let $W_{r}$ be a linear subspace of $B_{r}^{1}$. Let $G^{1} \in L^{2}\left((0, T) \times \Omega_{r \pm}\right)$ be a vector, $G^{2} \in L^{2}\left((0, T) \times \Omega_{r_{ \pm}}\right)$be a matrix, $G^{3} \in L^{2}\left((0, T) \times \Gamma_{r}\right)$ be a scalar and $G^{4} \in L^{2}\left((0, T) \times \Gamma_{r}\right)$ be a 2-vector. Suppose that $v \in X \cap W_{r}$ is such that

$$
\begin{aligned}
& \left(\partial_{t} v, w\right)_{B_{r}^{0}(t)}+\mu\{v, w\}_{B_{r}^{1}(t)}+\sum_{ \pm}\left(A^{2}(v, \nabla v), w\right)_{L^{2}\left(\Omega_{r \pm}\right)} \\
& =\sum_{ \pm}\left(G^{1}, w\right)_{L^{2}\left(\Omega_{r \pm}\right)}+\sum_{ \pm}\left(G^{2}, \nabla w\right)_{L^{2}\left(\Omega_{r \pm}\right)}+\left(G^{3}, w_{3}\right)_{L^{2}\left(\Gamma_{r}\right)} \\
& \quad+\left(G^{4}, \nabla^{\prime} w_{3}\right)_{L^{2}\left(\Gamma_{r}\right)}
\end{aligned}
$$

for all $w \in W_{r}$ for almost every $t \in(0, T)$. Then we have the estimates

$$
\begin{aligned}
& \underset{\tau \in(0, t)}{\operatorname{ess} \sup }\|v\|_{B_{r}^{0}(\tau)}^{2} \leqq C_{\infty, 0}\left(\|v(0)\|_{B_{r}^{0}}^{2}\right. \\
& \left.+\int_{0}^{t} \sum_{ \pm}\left(\left\|G^{1}\right\|_{L^{2}\left(\Omega_{r \pm}\right)}^{2}+\left\|G^{2}\right\|_{L^{2}\left(\Omega_{r \pm}\right)}^{2}\right)+\left\|G^{3}\right\|_{L^{2}\left(\Gamma_{r}\right)}^{2}+\left\|G^{4}\right\|_{L^{2}\left(\Gamma_{r}\right)}^{2} \mathrm{~d} \tau\right) \\
& \times \int_{0}^{t}\|v\|_{B_{r}^{1}(\tau)}^{2} \mathrm{~d} \tau \\
& \leqq C_{\infty, 0} \int_{0}^{t}\|v(0)\|_{B_{r}^{0}}^{2}+\sum_{ \pm}\left(\left\|G^{1}\right\|_{L^{2}\left(\Omega_{r \pm}\right)}^{2}+\left\|G^{2}\right\|_{L^{2}\left(\Omega_{r \pm}\right)}^{2}\right)+\left\|G^{3}\right\|_{L^{2}\left(\Gamma_{r}\right)}^{2} \\
& +\left\|G^{4}\right\|_{L^{2}\left(\Gamma_{r}\right)}^{2} \mathrm{~d} \tau \text {. }
\end{aligned}
$$

Moreover, suppose that we have $G^{2} \in L^{\infty}\left((0, T) ; L^{2}\left(\Omega_{r_{ \pm}}\right)\right), \partial_{t} G^{2} \in$ $L^{2}\left((0, T) \times \Omega_{r \pm}\right), G^{3}, G^{4} \in L^{\infty}\left((0, T) ; L^{2}\left(\Gamma_{r}\right)\right), \partial_{t} G^{3}, \partial_{t} G^{4} \in L^{2}\left((0, T) \times \Gamma_{r}\right)$, $\partial_{t} v \in L^{2}\left((0, T) ; W_{r}\right)$, so that in particular $v \in C\left([0, T] ; B_{r}^{1}\right)$. Then

$$
\begin{aligned}
& \int_{0}^{t}\left\|\partial_{t} v\right\|_{B_{r}^{0}(\tau)}^{2} \mathrm{~d} \tau+\underset{\tau \in(0, t)}{\operatorname{ess} \sup \|v\|_{B_{r}^{1}(t)}^{2}} \\
& \quad \leqq \frac{C_{\infty, 1}}{\varepsilon} \int_{0}^{t}\|v(0)\|_{B_{r}^{0}}^{2}+\sum_{ \pm}\left(\left\|G^{1}\right\|_{L^{2}\left(\Omega_{r \pm}\right)}^{2}+\left\|G^{2}\right\|_{L^{2}\left(\Omega_{r \pm}\right)}^{2}\right)+\left\|G^{3}\right\|_{L^{2}\left(\Gamma_{r}\right)}^{2} \\
& \quad+\left\|G^{4}\right\|_{L^{2}\left(\Gamma_{r}\right)}^{2} \mathrm{~d} \tau \\
& \quad+C_{\infty, 0}\left(\|v(0)\|_{B_{r}^{1}}^{2}+\sum_{ \pm} \underset{\tau \in(0, t)}{\left.\operatorname{ess~sup}\left(\left\|G^{2}\right\|_{L^{2}\left(\Omega_{r \pm}\right)}^{2}+\left\|G^{3}\right\|_{L^{2}\left(\Gamma_{r}\right)}^{2}+\left\|G^{4}\right\|_{L^{2}\left(\Gamma_{r}\right)}^{2}\right)\right)}\right. \\
& \quad+\varepsilon \int_{0}^{t} \sum_{ \pm}\left\|\partial_{t} G^{2}\right\|_{L^{2}\left(\Omega_{r \pm}\right)}^{2}+\left\|\partial_{t} G^{3}\right\|_{L^{2}\left(\Gamma_{r}\right)}^{2}+\left\|\partial_{t} G^{4}\right\|_{L^{2}\left(\Gamma_{r}\right)}^{2} \mathrm{~d} \tau
\end{aligned}
$$

where we are free to choose $\varepsilon \in(0,1]$.

Proof. To prove (147) we test the equation with $w=v \in W_{r}$. We then use the product rule, together with Cauchy's inequality and the fact that $\|v\|_{H^{1}\left(\Omega_{r \pm}\right)}+$ $\left\|v_{3}\right\|_{H^{1}\left(\Gamma_{r}\right)} \leqq C_{\infty, 0}\|v\|_{B_{r}^{1}(t)}$ as proved in Proposition 17 to obtain 


$$
\begin{aligned}
& \|v\|_{B_{r}^{0}(t)}^{2}+2 \mu \int_{0}^{t}\|v\|_{B_{r}^{1}(\tau)}^{2} \mathrm{~d} \tau \leqq C_{\infty, 0} \int_{0}^{t}\|v\|_{B_{r}^{0}(\tau)} \mathrm{d} \tau+\|v(0)\|_{B_{r}^{0}}^{2} \\
& +C_{\infty, 0} \int_{0}^{t} \sum_{ \pm}\left\|G^{1}\right\|_{L^{2}\left(\Omega_{r \pm}\right)}^{2}+\sum_{ \pm}\left\|G^{2}\right\|_{L^{2}\left(\Omega_{r \pm}\right)}^{2}+\left\|G^{3}\right\|_{L^{2}\left(\Gamma_{r}\right)}^{2}+\left\|G^{4}\right\|_{L^{2}\left(\Gamma_{r}\right)}^{2} \mathrm{~d} \tau
\end{aligned}
$$

Applying Gronwall's inequality we conclude (147). Returning to the above inequality and taking the supremum over $\tau \in(0, t)$ which allows us to absorb the term $\|v(0)\|_{B_{r}^{0}}^{2}$ into the left hand side, then using (147), we obtain (148). This proves the first part of the result.

We now assume the additional regularity as stated in the second part of the proposition. Since we have $\partial_{t} v \in W_{r}$ for almost every $t \in(0, T)$, we may set $w=\partial_{t} v$ in the equation. We use the product rule on the term $\left\{v, \partial_{t} v\right\}_{B_{r}^{1}(t)}$ then integrate in time, use Cauchy's inequality and integration by parts in time where necessary to conclude (149).

Proposition 19. Let $1 \leqq r<\infty$.

Let $G^{1} \in L^{2}\left((0, T) \times \Omega_{r_{ \pm}}\right)$be a vector, $G^{2} \in L^{\infty}\left((0, T) ; L^{2}\left(\Omega_{r \pm}\right)\right)$ with $\partial_{t} G^{2} \in L^{2}\left((0, T) \times \Omega_{r \pm}\right)$ be a matrix, $G^{3} \in L^{\infty}\left((0, T) ; L^{2}\left(\Gamma_{r}\right)\right)$ with $\partial_{t} G^{3} \in L^{2}\left((0, T) \times \Gamma_{r}\right)$ be a scalar and $G^{4} \in L^{\infty}\left((0, T) ; L^{2}\left(\Gamma_{r}\right)\right)$ with $\partial_{t} G^{4} \in L^{2}\left((0, T) \times \Gamma_{r}\right)$ be a 2-vector. Then there exists a unique $v \in X_{r}$ with

$$
v(0)=0
$$

such that

$$
\begin{aligned}
& \left(\partial_{t} v, w\right)_{B_{r}^{0}(t)}+\mu\{v, w\}_{B_{r}^{1}(t)}+\sum_{ \pm}\left(A^{2}(v, \nabla v), w\right)_{L^{2}\left(\Omega_{r \pm}\right)} \\
& =\sum_{ \pm}\left(G^{1}, w\right)_{L^{2}\left(\Omega_{r \pm}\right)}+\sum_{ \pm}\left(G^{2}, \nabla w\right)_{L^{2}\left(\Omega_{r \pm}\right)}+\left(G^{3}, w_{3}\right)_{L^{2}\left(\Gamma_{r}\right)} \\
& \quad+\left(G^{4}, \nabla^{\prime} w_{3}\right)_{L^{2}\left(\Gamma_{r}\right)}
\end{aligned}
$$

for all $w \in B_{r}^{1}$ for almost every $t \in(0, T)$, and $v$ satisfies the estimate (149).

Proof. Firstly, we claim that for each integer $m \geqq 1$, there exist functions $d_{m}^{1}, \ldots, d_{m}^{m} \in H^{1}(0, T)$ such that

$$
v_{m}:=\sum_{k=1}^{m} d_{m}^{k}(t) z_{k} \in H^{1}\left((0, T) ; B_{r}^{1}\right)
$$

satisfies the Equation (150) for all test functions $w \in W_{r}^{m}:=\left\langle z_{1}, \ldots, z_{m}\right\rangle$, and also $\left.v_{m}\right|_{t=0}=0$, where $\left(z_{k}\right)_{k=1}^{\infty}$ is the Galerkin basis defined in Lemma 19. Explicitly,

$$
\left.v_{m}\right|_{t=0}=0,
$$

and the following holds for almost every $t \in(0, T)$ :

$$
\left(\partial_{t} v_{m}, w\right)_{B_{r}^{0}(t)}+\mu\left\{v_{m}, w\right\}_{B_{r}^{1}(t)}+\sum_{ \pm}\left(A^{2}\left(v_{m}, \nabla v_{m}\right), w\right)_{L^{2}\left(\Omega_{r \pm}\right)}
$$




$$
\begin{aligned}
= & \sum_{ \pm}\left(G^{1}, w\right)_{L^{2}\left(\Omega_{r \pm}\right)}+\sum_{ \pm}\left(G^{2}, \nabla w\right)_{L^{2}\left(\Omega_{r \pm}\right)}+\left(G^{3}, w_{3}\right)_{L^{2}\left(\Gamma_{r}\right)} \\
& +\left(G^{4}, \nabla^{\prime} w_{3}\right)_{L^{2}\left(\Gamma_{r}\right)}
\end{aligned}
$$

for all $w \in W_{r}^{m}:=\left\langle z_{1}, \ldots, z_{m}\right\rangle$.

Indeed, we define $\left(d_{m}^{1}, \ldots, d_{m}^{m}\right)$ as the solution of the following system:

$$
\begin{aligned}
& \sum_{k=1}^{m} \frac{\mathrm{d} d_{m}^{k}}{\mathrm{~d} t}(t)\left(z_{k}, z_{l}\right)_{B_{r}^{0}(t)} \\
& \quad+\sum_{k=1}^{m} d_{m}^{k}(t)\left(\mu\left\{z_{k}, z_{l}\right\}_{B_{r}^{1}(t)}+\sum_{ \pm}\left(A^{2}\left(z_{k}, \nabla z_{k}\right), z_{l}\right)_{L^{2}\left(\Omega_{r \pm}\right)}\right) \\
& =\sum_{ \pm}\left(G^{1}, z_{l}\right)_{L^{2}\left(\Omega_{r \pm}\right)}+\sum_{ \pm}\left(G^{2}, \nabla z_{l}\right)_{L^{2}\left(\Omega_{r \pm}\right)}+\left(G^{3},\left(z_{l}\right)_{3}\right)_{L^{2}\left(\Gamma_{r}\right)} \\
& \quad+\left(G^{4}, \nabla^{\prime}\left(z_{l}\right)_{3}\right)_{L^{2}\left(\Gamma_{r}\right)},
\end{aligned}
$$

for all $1 \leqq l \leqq m$ with $d_{m}^{k}(0)=0$ for all $k$. Note that we have shown in Lemma 18 that the matrix $(M(t))_{k l}:=\left(z_{k}, z_{l}\right)_{B_{r}^{0}(t)}$ multiplying the vector $d_{m}$ is invertible with inverse in $W^{1, \infty}(0, T)$, hence we can write this as a standard first-order linear system of ODEs of the form

$$
\frac{\mathrm{d} d_{m}}{\mathrm{~d} t}=A(t) d_{m}+b(t)
$$

where $A(t) \in W^{1, \infty}(0, T), b(t) \in L^{2}(0, T)$. Hence, by standard theory, a solution $d_{m}=\left(d_{m}^{1}, \ldots, d_{m}^{m}\right)$ exists on the interval $(0, T)$ with $d_{m}^{1}, \ldots, d_{m}^{m} \in H^{1}(0, T)$.

We then define

$$
v_{m}:=\sum_{k=1}^{m} d_{m}^{k}(t) z_{k}
$$

It is clear by the construction of $d_{m}^{k}$ and linearity that $v_{m}$ satisfies the Equation (151) for all test functions $w \in\left\langle z_{1}, \ldots, z_{m}\right\rangle$, and also $\left.v_{m}\right|_{t=0}=0$.

This proves the claim. We also note that, if, in addition, we are given that $\partial_{t} G^{1} \in L^{2}\left((0, T) \times \Omega_{ \pm}\right)$, then $b(t) \in H^{1}(0, T)$ and hence $d_{m}^{1}, \ldots, d_{m}^{m} \in H^{2}(0, T)$ and $v_{m} \in H^{2}\left((0, T) ; B_{r}^{1}\right)$.

Note that since $v_{m}, \partial_{t} v_{m} \in W_{r}^{m}$, and given the regularity of the $G^{i}$, we see that $v_{m}$ satisfies the estimate (149). Using this energy estimate, we can now prove the existence of a weak solution. Indeed, using weak-* compactness, there exists a $v \in X$ and a subsequence $v_{m_{l}}$ such that

$$
\begin{gathered}
v_{m_{l}} \stackrel{*}{\rightarrow} v \quad \text { in } \quad L^{\infty}\left((0, T) ; B^{1}\right) \\
\partial_{t} v_{m_{l}} \stackrel{*}{\rightarrow} \partial_{t} v \quad \text { in } \quad L^{2}\left((0, T) ; B^{0}\right) .
\end{gathered}
$$


We also have by weak-* lower semi-continuity of norms that $v$ satisfies the same estimate. We claim that $v$ is a weak solution of the equation. Indeed, let $w \in$ $C^{1}\left([0, T] ; B_{r}^{1}\right)$ be a function of the form

$$
w=\sum_{k=1}^{M} a^{k}(t) z_{k}
$$

Pick $L$ such that $m_{l} \geqq M$ for all $l \geqq L$. Then, by linearity, we have that the equation (151) holds for $v_{m_{l}}$ with test function $w(t)$, for all $l \geqq L$ and almost every $t$. We integrate from 0 to $T$, then let $l \rightarrow \infty$ and use the weak/weak- $*$ convergences above. We obtain that the time-integrated equation holds for $v$ with test function $w$. Since $\left(z_{k}\right)_{k=1}^{\infty}$ are dense in $B_{r}^{1}$, we may approximate any test function $w \in L^{2}\left([0, T] ; B_{r}^{1}\right)$ with functions of this form, thus obtaining that the time-integrated equation holds for $v$ with any test function $w \in L^{2}\left([0, T] ; B_{r}^{1}\right)$. In particular, we have that the equation (150) holds for $v$ for all $w \in B_{r}^{1}$ for almost every $t$. This proves that $v$ is a weak solution of the equation. It is straightforward and standard to show that $v(0)=0$, and uniqueness follows easily by applying the energy estimate (147) to the difference of two solutions.

Proposition 20. The previous proposition holds in the case $r=\infty$.

Proof. This is achieved in a straightforward fashion by setting

$$
\begin{aligned}
& G_{r}^{1}=\phi_{r} G^{1}+G^{2} \nabla \phi_{r}, \quad G_{r}^{2}=\phi_{r} G^{2} \\
& G_{r}^{3}=\phi_{r} G^{3}+\nabla^{\prime} \psi_{r} \cdot G^{4}, \quad G_{r}^{4}=\phi_{r} G^{4},
\end{aligned}
$$

where $\phi_{r}$ is such that $\phi_{r} \in C_{c}^{\infty}\left(B_{r}(0)\right)$ with $\phi_{r}=1$ on $B_{\frac{r}{2}}(0)$ and $\left|\nabla \phi_{r}\right| \leqq C$ where $C$ is independent of $r$. We may apply the previous result to obtain a weak solution $v_{r} \in X_{r}$ to the equation with $G_{r}^{i}$ replacing $G^{i}$ for $1 \leqq i \leqq 4$, and then we may extend $v_{r}$ by zero to obtain $v_{r \pm}(t) \in B^{1}$ for almost every $t$. We then use the energy estimate (149) and weak-* compactness to obtain a solution $v \in X$ with the desired properties. The proof of uniqueness is the same as for $r<\infty$.

Proposition 21. Let $1 \leqq r<\infty$. Suppose the assumptions of Proposition 19 hold, and let $v \in X_{r}$ be the unique weak solution given by this proposition.

Suppose in addition that $G^{1} \in L^{\infty}\left((0, T) ; L^{2}\left(\Omega_{r \pm}\right)\right), \partial_{t} G^{1} \in L^{2}((0, T) \times$ $\left.\Omega_{r \pm}\right)$ and that $\left.G^{2}\right|_{t=0} \in H^{1}\left(\Omega_{r \pm}\right)$. Let us write

$$
\sum_{ \pm}\left(\left.G^{1}\right|_{t=0}, w\right)_{L^{2}\left(\Omega_{r \pm}\right)}-\sum_{ \pm}\left(\left.\nabla \cdot G^{2}\right|_{t=0}, w\right)_{L^{2}\left(\Omega_{r \pm}\right)}=(h, w)_{B_{r}^{0}}
$$

so that $h \in B_{r}^{0}$ is given by

$$
h=\frac{\left|\underset{\sim}{n^{0}}\right|^{2}}{\rho_{\mu}^{0}}\left(\left.G^{1}\right|_{t=0}-\left.\nabla \cdot G^{2}\right|_{t=0}\right)
$$


and suppose that

$$
-\sum_{ \pm}( \pm)\left(\left.G_{3}^{2}\right|_{t=0}, w_{3}\right)_{L^{2}\left(\Gamma_{r}\right)}+\left(\left.G^{3}\right|_{t=0}, w_{3}\right)_{L^{2}\left(\Gamma_{r}\right)}+\left(\left.G^{4}\right|_{t=0}, \nabla^{\prime} w_{3}\right)_{L^{2}\left(\Gamma_{r}\right)}=0
$$

for all $w \in B_{r}^{1}$.

Then in fact we have

$$
\begin{aligned}
& \partial_{t} v \in L^{\infty}\left((0, T) ; B_{r}^{0}\right) \\
& \partial_{t} v \in L^{2}\left((0, T) ; B_{r}^{1}\right)
\end{aligned}
$$

with the estimate

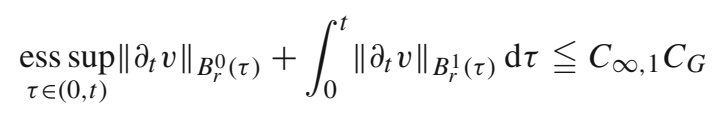

where $C_{G}$ denotes a constant depending in an affine manner on the appropriate norms of $G^{i}$.

Finally, suppose that we have the extra regularity $\partial_{t} G^{2} \in L^{\infty}\left((0, T) ; L^{2}\left(\Omega_{r \pm}\right)\right)$, $\partial_{t}^{2} G^{2} \in L^{2}\left((0, T) \times \Omega_{r \pm}\right), \partial_{t} G^{3}, \partial_{t} G^{4} \in L^{\infty}\left((0, T) ; L^{2}\left(\Gamma_{r}\right)\right), \partial_{t}^{2} G^{3}, \partial_{t}^{2} G^{4} \in$ $L^{2}\left((0, T) \times \Gamma_{r}\right)$. Suppose also that $h \in B_{r}^{1}$.

Then in fact we have

$$
\begin{aligned}
\partial_{t} v & \in L^{\infty}\left((0, T) ; B_{r}^{1}\right) \\
\partial_{t}^{2} v & \in L^{2}\left((0, T) ; B_{r}^{0}\right) \\
\left.\partial_{t} v\right|_{t=0} & =h
\end{aligned}
$$

with the estimate

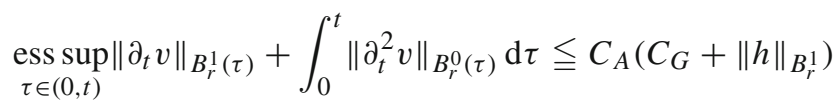

where $C_{G}$ denotes a constant depending in an affine manner on the appropriate norms of $G^{i}$. Moreover, $\partial_{t} v$ satisfies the equation

$$
\begin{aligned}
& \left(\partial_{t}^{2} v, w\right)_{B_{r}^{0}(t)}+\mu\left\{\partial_{t} v, w\right\}_{B_{r}^{1}(t)}+\sum_{ \pm}\left(A^{2}\left(\partial_{t} v, \nabla \partial_{t} v\right), w\right)_{L^{2}\left(\Omega_{r \pm}\right)} \\
& =\sum_{ \pm}\left(G^{1,1}, w\right)_{L^{2}\left(\Omega_{r \pm}\right)}+\sum_{ \pm}\left(G^{2,1}, \nabla w\right)_{L^{2}\left(\Omega_{r \pm}\right)}+\left(G^{3,1}, w_{3}\right)_{L^{2}\left(\Gamma_{r}\right)} \\
& \quad+\left(G^{4,1}, \nabla^{\prime} w_{3}\right)_{L^{2}\left(\Gamma_{r}\right)}
\end{aligned}
$$

for all $w \in B_{r}^{1}$ for almost every $t \in(0, T)$, where we have defined

$$
\begin{aligned}
G^{1,1} & :=\partial_{t} G^{1}-\partial_{t} A^{0} \partial_{t} v-\mu \partial_{t} A^{1}(v, \nabla v)-\partial_{t} A^{2}(v, \nabla v) \\
G^{2,1} & :=\partial_{t} G^{3}-\mu \partial_{t} A^{1}(v, \nabla v) \\
G^{3,1} & :=\partial_{t} G^{3} \\
G^{4,1} & :=\partial_{t} G^{4} .
\end{aligned}
$$

Note that in the first two lines above $\partial_{t} A^{1}(v, \nabla v)$ should be understood to mean the appropriate part or row of the full tensor $\partial_{t} A^{1}(v, \nabla v)$. 
Proof. We let $v_{m}$ be the same sequence of approximate solutions as in Proposition 19.

Note that since $\partial_{t} G^{1} \in L^{2}\left((0, T) \times \Omega_{r \pm}\right)$, we know from the construction of the Galerkin basis in Proposition 19 that $v_{m} \in H^{2}\left((0, T) ; B_{r}^{1}\right)$. This allows us to differentiate the Equation (151) with respect to $t$. One can then apply the energy estimates (147)-(148) to $\partial_{t} v_{m}$. We can prove $\left\|\left.\partial_{t} v_{m}\right|_{t=0}\right\|_{B_{r}^{0}} \leqq\|h\|_{B^{0}}$ by evaluating (151) at $t=0$, integrating by parts, and using the compatibility condition which we have assumed. One can then use weak-* compactness to pass to the limit $m \rightarrow \infty$ along a subsequence to obtain the first set of additional time regularity estimates for $v$.

Assuming the additional regularity properties stated in the proposition, we can apply the estimate (149) to $\partial_{t} v_{m}$. We use the condition $h \in B_{r}^{1}$ plus the special construction of our Galerkin basis to show that

$$
\left(\left.\partial_{t} v_{m}\right|_{t=0}, w\right)_{B_{r}^{1}}=(h, w)_{B_{r}^{1}}
$$

for all $w \in W_{r}^{m}$, and hence

$$
\left\|\left.\partial_{t} v_{m}\right|_{t=0}\right\|_{B_{r}^{1}} \leqq\|h\|_{B_{r}^{1}} .
$$

One can then use weak- $*$ compactness to pass to the limit $m \rightarrow \infty$ along a subsequence to obtain the second set of additional time regularity estimates for $v$.

It is straightforward then to show that

$$
\left.\partial_{t} v\right|_{t=0}=h .
$$

Proposition 22. The previous proposition holds in the case $r=\infty$.

Proof. This follows straightforwardly from the above in the same way that Proposition 20 followed from Proposition 19.

Proposition 23. Suppose $v \in X$ satisfies

$$
\begin{aligned}
& \left(\partial_{t} v, w\right)_{B^{0}(t)}+\mu\{v, w\}_{B^{1}(t)}+\sum_{ \pm}\left(A^{2}(v, \nabla v), w\right)_{L^{2}\left(\Omega_{ \pm}\right)} \\
& =\sum_{ \pm}\left(G^{1}, w\right)_{L^{2}\left(\Omega_{ \pm}\right)}+\sum_{ \pm}\left(G^{2}, \nabla w\right)_{L^{2}\left(\Omega_{ \pm}\right)}+\left(G^{3}, w_{3}\right)_{L^{2}(\Gamma)}+\left(G^{4}, \nabla^{\prime} w_{3}\right)_{L^{2}(\Gamma)}
\end{aligned}
$$

for all $w \in B^{1}$ for almost every $t \in(0, T)$, where $G^{1}, G^{2}, G^{3}, G^{4}$ have the following regularity.

$$
\begin{aligned}
& G^{1} \in L^{2}\left((0, T) ; L^{2}\left(\Omega_{ \pm}\right)\right), \quad G^{2} \in L^{2}\left((0, T) ; H^{1}\left(\Omega_{ \pm}\right)\right) \\
& G^{3} \in L^{2}\left((0, T) ; L^{2}(\Gamma)\right), \quad G^{4} \in L^{2}\left((0, T) ; H^{1}(\Gamma)\right) .
\end{aligned}
$$

Assume also

$$
v(0) \in B^{1} .
$$


Then in fact

$$
\begin{aligned}
v & \in L^{2}\left((0, T) ; H^{2}\left(\Omega_{ \pm}\right)\right) \\
v_{3} & \in L^{2}\left((0, T) ; H^{2}(\Gamma)\right)
\end{aligned}
$$

with corresponding norms bounded by $C_{A}\left(C_{G}+\|v(0)\|_{B^{1}}\right)$. In particular, the following estimate holds for any $\varepsilon \in(0,1]$.

$$
\begin{aligned}
& \left\|\nabla^{\prime} v\right\|_{B^{0}(t)}^{2}+2 \mu \int_{0}^{t}\left\|\nabla^{\prime} v\right\|_{B^{1}(\tau)}^{2} \mathrm{~d} \tau \leqq C\|v(0)\|_{B^{1}(t)}^{2} \\
& +C_{\infty, 1} \int_{0}^{t} \sum_{ \pm}\left\|G^{1}\right\|_{L^{2}\left(\Omega_{ \pm}\right)}^{2}+\sum_{ \pm}\left\|G^{2}\right\|_{H^{1}\left(\Omega_{ \pm}\right)}^{2}+\left\|G^{3}\right\|_{L^{2}(\Gamma)}^{2}+\left\|G^{4}\right\|_{H^{1}(\Gamma)}^{2} \mathrm{~d} \tau \\
& +\frac{C_{\infty, 1}}{\varepsilon} \int_{0}^{t}\|v\|_{H^{1}\left(\Omega_{ \pm}\right)}^{2} \mathrm{~d} \tau+\varepsilon \int_{0}^{t}\left\|\partial_{t} v\right\|_{L^{2}\left(\Omega_{ \pm}\right)}^{2} \mathrm{~d} \tau
\end{aligned}
$$

In addition, the following equations are satisfied:

$$
\begin{aligned}
& \rho_{\mu} \frac{1}{|\underset{\sim}{\mid n}|^{2}} \partial_{t} v-\mu N^{T} \nabla^{\psi}\left(\rho c^{2} \nabla^{\psi} \cdot(N v)\right)+\mu N^{T} \nabla^{\psi} \times\left(\rho c^{2} \nabla^{\psi} \times(N v)\right) \\
& \left.\quad+\rho_{\mu} N^{T} \overline{\left(u^{\psi}\right.} \cdot \nabla\right)(N v)+\rho_{\mu} N^{T}\left(\partial_{t} N\right) v=G^{1}-\nabla \cdot G^{2}
\end{aligned}
$$

almost everywhere in $(0, T) \times \Omega_{ \pm}$and

$$
\begin{aligned}
\mu\left(\Delta^{\prime}-1\right) v_{3}+\mu\left[\rho c^{2} \nabla^{\psi} \cdot(N v)\right] & =-\left[G_{33}^{2}\right]+G^{3}-\nabla^{\prime} \cdot G^{4} \\
\mu N^{T}\left(\rho_{ \pm} c_{ \pm}^{2} n \times\left(\nabla^{\psi} \times\left(N v_{ \pm}\right)\right)\right) & =-\left(G_{13 \pm}^{2}, G_{23 \pm}^{2}, 0\right)
\end{aligned}
$$

almost everywhere on $(0, T) \times \Gamma$.

Moreover, suppose for some $1 \leqq k \leqq 3$ that

$$
\begin{aligned}
& G^{1} \in L^{2}\left((0, T) ; H^{k}\left(\Omega_{ \pm}\right)\right), \quad G^{2} \in L^{2}\left((0, T) ; H^{k+1}\left(\Omega_{ \pm}\right)\right) \\
& G^{3} \in L^{2}\left((0, T) ; H^{k}(\Gamma)\right), \quad G^{4} \in L^{2}\left((0, T) ; H^{k+1}(\Gamma)\right) \\
& v(0) \in H^{k+1}\left(\Omega_{ \pm}\right), \quad v_{3}(0) \in H^{k+1}(\Gamma)
\end{aligned}
$$

and

$$
\partial_{t} v \in L^{2}\left((0, T) ; H^{k}\left(\Omega_{ \pm}\right)\right) \cap L^{\infty}\left((0, T) ; H^{k-1}\left(\Omega_{ \pm}\right)\right) .
$$

Then $v \in L^{2}\left((0, T) ; H^{k+1}\left(\Omega_{ \pm}\right)\right) \cap L^{\infty}\left((0, T) ; H^{k}\left(\Omega_{ \pm}\right)\right), v_{3} \in L^{2}((0, T)$; $\left.H^{k+1}(\Gamma)\right)$ with corresponding norms bounded by

$$
C_{A}\left(C_{G}+\|v(0)\|_{H^{k+1}\left(\Omega_{ \pm}\right)}+\left\|v_{3}(0)\right\|_{H^{k+1}(\Gamma)}+\int_{0}^{T}\left\|\partial_{t} v\right\|_{H^{k}\left(\Omega_{ \pm}\right)} \mathrm{d} t\right)
$$


Proof. The proof is technical but straightforward using difference quotients and a standard energy estimate obtained by testing the weak equation with

$$
w=-D_{i}^{-h}\left(\zeta_{i}^{2} D_{i}^{h} v\right)
$$

for $|h| \neq 0$ sufficiently small and $1 \leqq i \leqq 3$, where $D_{i}^{h}$ is standard notation for a difference quotient of step-size $h$ in the $x_{i}$-direction, $\zeta_{3}$ is a cut-off function which is zero near $x_{3}=0$ to exclude boundary terms and $\zeta_{1}, \zeta_{2}$ are equal to 1 . For the higher order regularity we use an inductive argument and the Sobolev embedding theorem. Note that the compatibility condition is hidden in the requirement $v(0) \in B^{1}$. This completes the proof.

12.7. Proof of Existence, Uniqueness and Regularity of Solutions of the Equations for the Rotated Velocity

Definition 43. We say $\stackrel{\vec{u}}{\text { is a }}$ a weak solution of the rotated equations for the velocity if $\stackrel{2}{u}-\stackrel{2}{u} \in X$,

$$
\left.\stackrel{\imath}{u}\right|_{t=0}=\stackrel{\overbrace{}}{u}^{0}
$$

and the following holds for almost every $t \in(0, T)$.

$$
\begin{aligned}
& \left(\partial_{t} \stackrel{\widetilde{u}}{,} w\right)_{B^{0}(t)}+\mu\{\tilde{\tilde{u}}, w\}_{B^{1}(t)}+\sum_{ \pm}\left(A^{1}(\tilde{u}, \nabla \tilde{u}), w\right)_{L^{2}\left(\Omega_{ \pm}\right)} \\
& =\sum_{ \pm}\left(G^{1}, w\right)_{L^{2}\left(\Omega_{ \pm}\right)}+\sum_{ \pm}\left(G^{2}, \nabla w\right)_{L^{2}\left(\Omega_{ \pm}\right)}+\left(G^{3}, w_{3}\right)_{L^{2}(\Gamma)} \\
& \quad+\left(G^{4}, \nabla^{\prime} w_{3}\right)_{L^{2}(\Gamma)}
\end{aligned}
$$

for all $w \in B^{1}$. Note that we rely on the fact that $\left[\widetilde{u}_{3}^{0}\right]=0$, which is true due to the compatibility condition (45) at order zero, for the term $\{\tilde{u}, w\}_{B^{1}(t)}$ to be well-defined.

The functions $G^{1}, G^{2}, G^{3}$ and $G^{4}$ are defined such that

$$
\begin{aligned}
\sum_{ \pm}\left(G^{1}, w\right)_{L^{2}\left(\Omega_{ \pm}\right)}+\sum_{ \pm}\left(G^{2}, \nabla w\right)_{L^{2}\left(\Omega_{ \pm}\right)} & \\
= & \left(N^{T}\left(g_{1}+\partial_{x_{3}}\left(\frac{\left(-\nabla^{\prime} \psi, 1\right)}{J^{\psi}}\right) p-\mu \partial_{x_{3}}\left(\frac{\left(-\nabla^{\prime} \psi, 1\right)}{J^{\psi}}\right) \times \rho c^{2} \omega\right), w\right)_{L^{2}\left(\Omega_{ \pm}\right)} \\
& \quad+\left(p, \nabla^{\psi} \cdot(N w)\right)_{L^{2}\left(\Omega_{ \pm}\right)}+\mu\left(\rho c^{2} \omega, \nabla^{\psi} \times(N w)\right)_{L^{2}\left(\Omega_{ \pm}\right)} \\
G^{3}= & g_{2} \\
G^{4}= & \sigma \hat{n} .
\end{aligned}
$$

Note that $G^{i}$ have the form

$$
\begin{aligned}
G^{1} & =G\left(N, \nabla N, \nabla \psi, p, \omega, \rho c^{2}, g_{1}\right) \\
G^{2} & =G\left(N, \nabla \psi, p, \omega, \rho c^{2}\right)
\end{aligned}
$$




$$
\begin{aligned}
G^{3} & =g_{2} \\
G^{4} & =G\left(\nabla^{\prime} f\right)
\end{aligned}
$$

for $G$ a generic smooth function which may be scalar, vector, matrix valued etc.

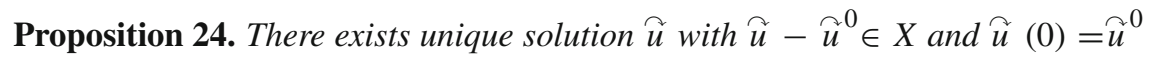
to the weak velocity equation above.

For $0 \leqq j \leqq 3$, we have $\partial_{t}^{j} \nabla \stackrel{\curvearrowright}{u} \in L^{\infty}\left((0, T) ; H^{3-j}\left(\Omega_{ \pm}\right)\right) \cap L^{2}((0, T)$;

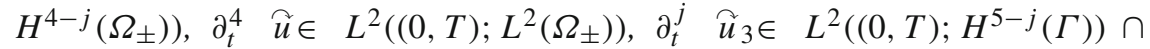
$L^{\infty}\left((0, T) ; H^{4-j}(\Gamma)\right)$, and, for $0 \leqq j \leqq 3$,

$$
\left.\partial_{t}^{j} \stackrel{\curvearrowright}{u}\right|_{t=0}=\partial_{0}^{\mu, g, j} \stackrel{\curvearrowright}{u}{ }^{0} .
$$

Moreover, $\overparen{u}$ is the unique solution to the equations for the rotated velocity with $\widetilde{u}(0)=\stackrel{\overbrace{}}{u}^{0}$ as defined in Definition 38.

Proof. This is straightforward, if technical, using propositions 20, 23 and 22 inductively applied in the correct order together with the Sobolev embedding theorem and the fact that the initial data satisfies the compatibility condition (45) up to order 3 and the compatibility condition (46) up to order 2 . The definition of $G^{1}, \ldots, G^{4}$ ensures that $\tilde{u}$ is the unique solution to the equations for the rotated velocity.

We now show the additional boundary regularity. We will use the elliptic estimate lemma, Lemma 11.

Lemma 20. For $0 \leqq j \leqq 3$, we have $\partial_{t}^{j} \overbrace{3} \in L^{2}\left((0, T) ; H^{5.5-j}(\Gamma)\right)$.

Proof. Applying $\partial_{t}^{j}$ to the pressure interface condition (143) we have

$$
\left(1-\Delta^{\prime}\right) \partial_{t}^{j} \widetilde{\sim}_{3}=\partial_{t}^{j}\left(\frac{1}{\mu}\left(-[p]-\sigma \nabla^{\prime} \cdot \hat{n}+g_{2}\right)+\left[\rho c^{2} \nabla^{\psi} \cdot(N \stackrel{\widetilde{u}}{)}]\right) .\right.
$$

We apply the Lemma 11 with

$$
\begin{aligned}
& z=\partial_{t}^{j} \stackrel{\curvearrowright}{u}_{3} \\
& h=\partial_{t}^{j}\left(\frac{1}{\mu}\left(-[p]-\sigma \nabla^{\prime} \cdot \hat{n}+g_{2}\right)+\left[\rho c^{2} \nabla^{\psi} \cdot(N \stackrel{\curvearrowright}{u})\right]\right)
\end{aligned}
$$

and the Sobolev trace estimate to conclude that for almost every $t, \partial_{t}^{j} \widetilde{u}_{3} \in$ $H^{5.5-j}(\Gamma)$ with

$$
\begin{aligned}
& \left\|\partial_{t}^{j} \stackrel{\sim}{u}_{3}\right\|_{H^{5.5-j}(\Gamma)}^{2} \\
& \leqq C \| \partial_{t}^{j}\left(\frac{1}{\mu}\left(-[p]-\sigma \nabla^{\prime} \cdot \hat{n}+g_{2}\right)+\left[\rho c^{2} \nabla^{\psi} \cdot(N \stackrel{\tilde{u}}{)}]\right) \|_{H^{3.5-j}(\Gamma)}^{2} .\right.
\end{aligned}
$$

Integrating from 0 to $t$ and using existing estimates together with the product rule, we obtain

$$
\int_{0}^{t}\left\|\partial_{t}^{j} \stackrel{\Im}{u}_{3}\right\|_{H^{5.5-j}(\Gamma)}^{2}<\infty
$$

This completes the proof. 
Applying Proposition 24, Lemma 20 and Proposition 16, we prove Proposition 15. Together with the results in the previous section, we have proved Proposition 12.

\section{Proof of the Energy Estimate for the Linearised Equations}

In this section we prove the energy estimate for the linearised equations stated in Proposition 11 . The energy estimate is necessary as part of the construction of a welldefined contraction map sending a background state to a solution of the linearised equations linearised about that state. The main steps in the energy estimate are quite clear. The front is estimated by direct integration and the entropy, pressure and curl are estimated in the standard manner of energy estimates for transport equations, with some minor differences in the curl estimate. The velocity is then estimated in a manner which is fairly standard for parabolic equations. We then obtain a slightly higher order estimate for $u \cdot n$ from the elliptic equation provided by the interface condition (134).

Our main task will be to show that

$$
\begin{aligned}
\mathcal{E}_{L}(t)+\mathcal{E}_{L}(\omega)(t) \leqq & \frac{1}{\varepsilon} M_{\mu}^{0}+\frac{1}{\varepsilon} K_{\mu}(t)\left(t+\int_{0}^{t} \overline{\mathcal{E}}_{L}(\tau)+\mathcal{E}_{L}(\tau)+\mathcal{E}_{L}(\omega)(\tau) \mathrm{d} \tau\right) \\
& +\varepsilon\left(M_{\mu}^{0}+t K_{\mu}(t)\right)\left(\overline{\mathcal{E}}_{L}(t)+\mathcal{E}_{L}(t)+\mathcal{E}_{L}(\omega)(t)\right)
\end{aligned}
$$

where we are free to choose $\varepsilon \in(0,1]$.

Let us also observe that we have

$$
\begin{aligned}
& \left.\partial_{t}^{j}\left(U_{+}, U_{-}, \omega_{+}, \omega_{-}, f\right)\right|_{t=0} \\
& \quad=\partial_{0}^{\mu, g, j}\left(U_{+}^{0}, U_{-}^{0}, \nabla^{\psi^{0}} \times u_{+}^{0}, \nabla^{\psi^{0}} \times u_{-}^{0}, f^{0}\right) \text { for } 0 \leqq j \leqq 3,
\end{aligned}
$$

even though we have only assumed the case $j=0$, which we can deduce in the same way as in the proof of Proposition 14.

\subsection{Lower Order Estimate}

Lemma 21. We have the following lower order estimates:

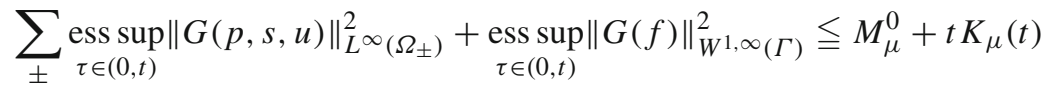

$$
\begin{aligned}
& \sum_{1 \leqq|\beta| \leqq 2} \operatorname{esssup}_{\tau \in(0, t)} \partial^{\beta}(p, s, u)\left\|_{L^{2}\left(\Omega_{ \pm}\right)}^{2}+\sum_{0 \leqq|\beta| \leqq 1} \operatorname{ess} \operatorname{esup}\right\| \partial^{\beta} \nabla u \|_{H^{1}\left(\Omega_{ \pm}\right)}^{2} \\
& \leqq M_{\mu}^{0}+t K_{\mu}(t) \\
& \sum_{0 \leqq|\beta| \leqq 2}\left\|\partial^{\beta} \nabla^{\prime} f\right\|_{H^{1.5}(\Gamma)}^{2} \leqq M_{\mu}^{0}+t K_{\mu}(t),
\end{aligned}
$$

where $G$ is a smooth function of its arguments.

Proof. These follow easily from the fundamental theorem of calculus and the chain rule. 


\subsection{Estimate of the Front}

Proposition 25. Define

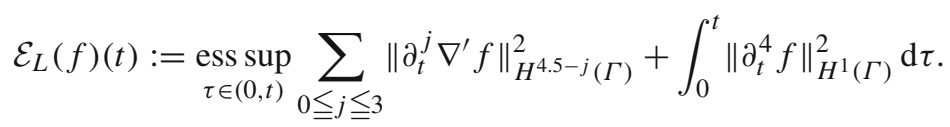

The front satisfies the following estimates for $t \in(0, T)$.

$$
\begin{aligned}
& \mathcal{E}_{L}(f)(t) \leqq M_{\mu}^{0}+\frac{C}{\varepsilon} \int_{0}^{t} \mathcal{E}_{L}(\tau) \mathrm{d} \tau+\varepsilon \overline{\mathcal{E}}_{L}(t) \\
& \underset{\tau \in(0, t)}{\operatorname{ess} \sup } \sum_{0 \leqq j \leqq 3}\left\|\partial_{t}^{j} \nabla^{\prime} f\right\|_{H^{3-j}(\Gamma)}^{2}+\sum_{0 \leqq j \leqq 3} \int_{0}^{t}\left\|\partial_{t}^{j+1} \nabla^{\prime} f\right\|_{H^{3-j}(\Gamma)}^{2} \mathrm{~d} \tau \\
& \leqq M_{\mu}^{0}+C \int_{0}^{t} \overline{\mathcal{E}}_{L}(\tau) \mathrm{d} \tau \\
& \sum_{0 \leqq j \leqq 3} \int_{0}^{t}\left\|\partial_{t}^{j+1} f\right\|_{H^{5.5-j}(\Gamma)}^{2} \mathrm{~d} \tau+\sum_{0 \leqq j \leqq 3} \operatorname{ess~sup}\left\|\partial_{t}^{j+1} f\right\|_{H^{4-j}(\Gamma)}^{2} \leqq C \overline{\mathcal{E}}_{L}(t)
\end{aligned}
$$

Moreover, we have

$$
J^{\psi} \geqq \kappa^{0}-t K_{\mu}(t)
$$

Proof. This is straightforward using the fact that $\partial_{t} f=\bar{u} \cdot \bar{n}$, the definition of the energy $\mathcal{E}_{L}(t)$, plus Cauchy's inequality for the first estimate. For the final inequality we use the fundamental theorem of calculus.

\subsection{Estimate of the Entropy, Pressure and Curl}

We start with an estimate for general transport equations of the correct form.

Lemma 22. In this lemma functions will be defined either on $\Omega_{+}$or $\Omega_{-}$.

Suppose we are given $b \in L^{\infty}\left((0, T) ; W^{1, \infty}\left(\Omega_{ \pm} ; \mathbb{R}^{3}\right)\right), h \in L^{2}\left((0, T) ; L^{2}\left(\Omega_{ \pm}\right)\right)$ and $w \in L^{2}\left((0, T) ; H^{1}\left(\Omega_{ \pm}\right)\right)$with $\partial_{t} w \in L^{2}\left((0, T) ; L^{2}\left(\Omega_{ \pm}\right)\right)$. Suppose also that $\left.b_{3}\right|_{\Gamma}=0$ and that $w$ satisfies the equation

$$
\left(\partial_{t}+b \cdot \nabla\right) w=h
$$

in $(0, T) \times \Omega_{ \pm}$. Then $w$ satisfies the following estimates, where we are free to choose $\varepsilon \in(0,1]$.

$$
\begin{aligned}
\underset{\tau \in(0, t)}{\operatorname{ess} \sup \|w\|_{L^{2}\left(\Omega_{ \pm}\right)}^{2}} & =\|w(0)\|_{L^{2}\left(\Omega_{ \pm}\right)}^{2}+\left(\frac{1}{\varepsilon}+\underset{\tau \in(0, t)}{\left.\operatorname{ess} \sup \|b\|_{W^{1, \infty}\left(\Omega_{ \pm}\right)}^{2}\right)}\right. \\
& \times \int_{0}^{t}\|w\|_{L^{2}\left(\Omega_{ \pm}\right)}^{2} \mathrm{~d} \tau+\varepsilon \int_{0}^{t}\|h\|_{L^{2}\left(\mathbb{R}^{d}\right)}^{2} \mathrm{~d} \tau
\end{aligned}
$$




$$
\begin{aligned}
\int_{0}^{t}\left\|\partial_{t} w\right\|_{L^{2}\left(\Omega_{ \pm}\right)}^{2} \mathrm{~d} \tau \leqq & \underset{\tau \in(0, t)}{\operatorname{ess} \sup \|b\|_{W^{1, \infty}\left(\Omega_{ \pm}\right)}^{2}} \int_{0}^{t}\|\nabla w\|_{L^{2}\left(\Omega_{ \pm}\right)}^{2} \mathrm{~d} \tau \\
& +C \int_{0}^{t}\|h\|_{L^{2}\left(\Omega_{ \pm}\right)}^{2} \mathrm{~d} \tau .
\end{aligned}
$$

Moreover, if $h \in L^{2}\left((0, T) ; H^{1}\left(\Omega_{ \pm}\right)\right)$and $w(0) \in H^{1}\left(\Omega_{ \pm}\right)$then

$$
\begin{aligned}
& \underset{\tau \in(0, t)}{\operatorname{ess} \sup \|w\|_{H^{1}\left(\Omega_{ \pm}\right)}^{2} \leqq\|w(0)\|_{H^{1}\left(\Omega_{ \pm}\right)}^{2}}+\left(\frac{1}{\varepsilon}+\underset{\tau \in(0, t)}{\left.\operatorname{ess} \sup \|b\|_{W^{1, \infty}\left(\Omega_{ \pm}\right)}^{2}\right)} \int_{0}^{t}\|w\|_{H^{1}\left(\mathbb{R}^{d}\right)}^{2} \mathrm{~d} \tau\right. \\
& +\varepsilon \int_{0}^{t}\|h\|_{H^{1}\left(\Omega_{ \pm}\right)}^{2} \mathrm{~d} \tau,
\end{aligned}
$$

where we are free to choose $\varepsilon \in(0,1]$.

Proof. The first is a standard energy estimate with the crucial point being that $\left.b_{3}\right|_{\Gamma}=0$, and the third estimate can be proved by differentiating with respect to $x_{i}$ then using the first. The second is obtained simply by rearranging the equation.

Lemma 23. We have that $\overline{u^{\psi}}$ satisfies the following estimate:

$$
\left\|\partial^{\gamma} \overline{u^{\psi}}\right\|_{L^{\infty}\left(\Omega_{ \pm}\right)}^{2} \leqq K_{\mu}(t) \text { for } 0 \leqq|\gamma| \leqq 1
$$

Proof. Let us note that

$$
\overline{u^{\psi}}=G\left(\partial_{t} \psi, \nabla \psi, \nabla^{\prime} \bar{\psi}, \bar{u}\right)
$$

for some smooth function $G$ (at least in the region where $J^{\psi}>0$ ). The estimate follows immediately using the chain rule and the Sobolev embedding theorem.

Proposition 26. Define

$$
\begin{aligned}
\mathcal{E}_{L}(s)(t):= & \sum_{ \pm} \operatorname{ess} \sup \left(\|s\|_{L^{\infty}\left(\Omega_{ \pm}\right)}^{2}+\sum_{1 \leqq|\alpha| \leqq 3}\left\|\partial^{\alpha} s\right\|_{H^{1}\left(\Omega_{ \pm}\right)}^{2}\right) \\
& +\sum_{ \pm} \int_{0}^{t}\left\|\partial_{t}^{4} s\right\|_{L^{2}\left(\Omega_{ \pm}\right)}^{2} \mathrm{~d} \tau .
\end{aligned}
$$

The entropy s satisfies the following energy estimate:

$$
\mathcal{E}_{L}(s)(t) \leqq M_{\mu}^{0}+K_{\mu}(t)\left(\int_{0}^{t} \mathcal{E}_{L}(\tau)+\overline{\mathcal{E}}_{L}(\tau) \mathrm{d} \tau\right)
$$

for $t \in(0, T)$. 
Proof. Let $1 \leqq|\alpha| \leqq 3$ and apply $\partial^{\alpha}$ to the linearised entropy equation (128) to obtain

$$
\partial_{t} \partial^{\alpha} s+\overline{u^{\psi}} \cdot \nabla \partial^{\alpha} s=R_{L}^{\alpha}(s)
$$

where

$$
R_{L}^{\alpha}(s)=-\sum_{\beta+\gamma=\alpha, \gamma \neq \alpha} \partial^{\beta} \overline{u^{\psi}} \cdot \partial^{\gamma} \nabla s .
$$

One can easily show using the Sobolev embedding theorem and Holder's inequality that

$$
\left\|R_{L}^{\alpha}(s)\right\|_{H^{1}\left(\Omega_{ \pm}\right)}^{2} \leqq K_{\mu}(t)\left(\overline{\mathcal{E}}_{L}(t)+\mathcal{E}_{L}(t)\right) .
$$

Now we use the estimates (165) and (164) to conclude the result.

Proposition 27. Define

$$
\begin{aligned}
\mathcal{E}_{L}(p)(t):= & \sum_{ \pm} \underset{\tau \in(0, t)}{\operatorname{ess} \sup }\left(\|p\|_{L^{\infty}\left(\Omega_{ \pm}\right)}^{2}+\sum_{1 \leqq|\alpha| \leqq 3}\left\|\partial^{\alpha} p\right\|_{H^{1}\left(\Omega_{ \pm}\right)}^{2}\right) \\
& +\sum_{ \pm} \int_{0}^{t}\left\|\partial_{t}^{4} p\right\|_{L^{2}\left(\Omega_{ \pm}\right)}^{2} \mathrm{~d} \tau .
\end{aligned}
$$

The pressure p satisfies the following energy estimate.

$$
\begin{aligned}
\mathcal{E}_{L}(p)(t) \leqq & M_{\mu}^{0}+\frac{1}{\varepsilon} K_{\mu}(t)\left(\int_{0}^{t} \mathcal{E}_{L}(\tau)+\overline{\mathcal{E}}_{L}(\tau) \mathrm{d} \tau\right)+\varepsilon\left(M_{\mu}^{0}+t K_{\mu}(t)\right)\left(\overline{\mathcal{E}}_{L}(t)\right. \\
& \left.+\mathcal{E}_{L}(t)\right)
\end{aligned}
$$

for $t \in(0, T)$, where we are free to choose $\varepsilon>0$.

Proof. Let $1 \leqq|\alpha| \leqq 3$ and apply $\partial^{\alpha}$ to the linearised pressure equation (129) to obtain

$$
\partial_{t} \partial^{\alpha} p+\overline{u^{\psi}} \cdot \nabla \partial^{\alpha} p=\partial^{\alpha} G_{p}+R_{L}^{\alpha}(p)
$$

where

$$
G_{p}:=-\bar{\rho} \bar{c}^{2} \nabla^{\psi} \cdot \bar{u}
$$

Note that we have

$$
\left\|R_{L}^{\alpha}(p)\right\|_{H^{1}\left(\Omega_{ \pm}\right)}^{2} \leqq K_{\mu}(t)\left(\overline{\mathcal{E}}_{L}(t)+\mathcal{E}_{L}(t)\right)
$$

exactly as in the estimate of the entropy. Using the Sobolev embedding theorem we can show that

$$
\begin{aligned}
\int_{0}^{t}\left\|\partial^{\alpha} G_{p}\right\|_{H^{1}\left(\Omega_{ \pm}\right)}^{2} \mathrm{~d} \tau & \leqq\left(M_{\mu}^{0}+t K_{\mu}(t)\right)\left(\overline{\mathcal{E}}_{L}(t)+\mathcal{E}_{L}(t)\right) \\
\int_{0}^{t}\left\|\partial^{\alpha} G_{p}\right\|_{L^{2}\left(\Omega_{ \pm}\right)}^{2} \mathrm{~d} \tau & \leqq K_{\mu}(t) \int_{0}^{t} \overline{\mathcal{E}}_{L}(t)+\mathcal{E}_{L}(t) \mathrm{d} \tau .
\end{aligned}
$$

Now we use the estimates (165) and (164) to conclude the result. 
Proposition 28. We have

$$
\begin{aligned}
& \rho_{\mu}(t, x) \geqq \delta^{0}-t K_{\mu}(t) \\
& \rho_{\mu}(t, x) \geqq \delta_{\mu}^{0}-t K_{\mu}(t) .
\end{aligned}
$$

Proof. We use the fundamental theorem of calculus and the Sobolev embedding theorem.

Proposition 29. The curl $\omega$ satisfies the following energy estimates.

$$
\begin{aligned}
\mathcal{E}_{L}(\omega)(t) \leqq & M_{\mu}^{0}+\frac{1}{\varepsilon} K_{\mu}(t)\left(t+\int_{0}^{t} \overline{\mathcal{E}}_{L}(t)+\mathcal{E}_{L}(t)+\mathcal{E}_{L}(\omega)(t) \mathrm{d} \tau\right. \\
& +\varepsilon\left(M_{\mu}^{0}+t K_{\mu}(t)\right)\left(\overline{\mathcal{E}}_{L}(t)+\mathcal{E}_{L}(t)+\mathcal{E}_{L}(\omega)(t)\right) \\
\int_{0}^{t}\left\|\partial_{t}^{3} \omega\right\|_{H^{1}\left(\Omega_{ \pm}\right)}^{2} \mathrm{~d} \tau \leqq & K_{\mu}(t)\left(t+\int_{0}^{t} \overline{\mathcal{E}}_{L}(t)+\mathcal{E}_{L}(t)+\mathcal{E}_{L}(\omega)(t) \mathrm{d} \tau\right) \\
\int_{0}^{t}\left\|\partial_{t}^{4} \omega\right\|_{L^{2}\left(\Omega_{ \pm}\right)}^{2} \mathrm{~d} \tau \leqq & \left(M_{\mu}^{0}+t K_{\mu}(t)\right)\left(1+\overline{\mathcal{E}}_{L}(t)+\mathcal{E}_{L}(t)+\mathcal{E}_{L}(\omega)(t)\right)
\end{aligned}
$$

for $t \in(0, T)$, where we are free to choose $\varepsilon>0$.

Proof. Let $1 \leqq|\alpha| \leqq 3$ and apply $\partial^{\alpha}$ to the linearised curl equation (130) to obtain

$$
\left(\partial_{t}+\overline{u^{\psi}} \cdot \nabla\right) \partial^{\alpha} \omega=\partial^{\alpha} G_{\omega}+R_{L}^{\alpha}(\omega)
$$

where

$$
R_{L}^{\alpha}(\omega)=-\sum_{\gamma+\delta=\alpha, \delta \neq \alpha} \partial^{\gamma} \overline{u^{\psi}} \cdot \partial^{\delta} \nabla \omega
$$

and

$$
G_{\omega}:=\frac{1}{\rho_{\mu}}\left(-\nabla^{\psi} \rho_{\mu} \times\left(\partial_{t}+\overline{u^{\psi}} \cdot \nabla\right) \bar{u}-\rho_{\mu}\left(\varepsilon_{i j k} \partial_{j}^{\psi} \bar{u}_{l} \partial_{l}^{\psi} \bar{u}_{k}\right)+\nabla^{\psi} \times g_{1}\right) .
$$

One can straightforwardly show using the Sobolev embedding theorem and Holder's inequality that

$$
\left\|R_{L}^{\alpha}(\omega)\right\|_{H^{1}\left(\Omega_{ \pm}\right)}^{2} \leqq K_{\mu}(t)\left(\overline{\mathcal{E}}_{L}(t)+\mathcal{E}_{L}(t)+\mathcal{E}_{L}(\omega)(t)\right) .
$$

Using the Sobolev embedding theorem we can show that

$$
\int_{0}^{t}\left\|\partial^{\alpha} G_{\omega}\right\|_{L^{2}\left(\Omega_{ \pm}\right)}^{2} \mathrm{~d} \tau \leqq\left(M_{\mu}^{0}+t K_{\mu}(t)\right)\left(1+\overline{\mathcal{E}}_{L}(t)+\mathcal{E}_{L}(t)\right)
$$

and if $\alpha_{0} \leqq 2$, then

$$
\begin{aligned}
\int_{0}^{t}\left\|\partial^{\alpha} G_{\omega}\right\|_{H^{1}\left(\Omega_{ \pm}\right)}^{2} \mathrm{~d} \tau & \leqq\left(M_{\mu}^{0}+t K_{\mu}(t)\right)\left(1+\overline{\mathcal{E}}_{L}(t)+\mathcal{E}_{L}(t)\right) \\
\left\|\partial^{\alpha} G_{\omega}\right\|_{L^{2}\left(\Omega_{ \pm}\right)}^{2} & \leqq K_{\mu}(t)\left(1+\overline{\mathcal{E}}_{L}(t)+\mathcal{E}_{L}(t)\right) .
\end{aligned}
$$

Note that due to the fact that we only have $\partial_{t}^{4} u \in L^{2}\left((0, T) ; L^{2}\left(\Omega_{ \pm}\right)\right)$, the estimate for $G_{\omega}$ is not as good in the first case where we allow $\alpha_{0}=3$. Also, note that we use crucially the fact that we replaced $\rho$ by $\rho_{\mu}$ in the definition of the $\mu$-approximate velocity equation, which ensures that the term $\nabla^{\psi} \rho_{\mu}$ in $G_{\omega}$ can be easily estimated.

Now we use the estimates (165) and (164) to conclude the result. 


\subsection{Estimate of the Velocity}

Definition 44. We define the part of the energy $\mathcal{E}_{L}$ associated with the velocity $u$ for $t \in(0, T)$ by

$$
\begin{aligned}
& \mathcal{E}_{L}(u)(t):=\sum_{ \pm} \underset{\tau \in(0, t)}{\operatorname{ess} \sup \|u\|_{L^{\infty}\left(\Omega_{ \pm}\right)}^{2}}+\sum_{ \pm} \sum_{0 \leqq j \leqq 3} \operatorname{ess} \sup \left\|\partial_{t}^{j} \nabla u\right\|_{H^{3-j}\left(\Omega_{ \pm}\right)}^{2} \\
& +\sum_{ \pm} \sum_{0 \leqq j \leqq 3} \int_{0}^{t}\left\|\partial_{t}^{j} \nabla u\right\|_{H^{4-j}\left(\Omega_{ \pm}\right)}^{2} \mathrm{~d} \tau+\sum_{ \pm} \int_{0}^{t}\left\|\partial_{t}^{4} u\right\|_{L^{2}\left(\Omega_{ \pm}\right)}^{2} \mathrm{~d} \tau \\
& +\sum_{0 \leqq j \leqq 3} \underset{\tau \in(0, t)}{\operatorname{ess} \sup \|}\left\|\partial_{t}^{j}(u \cdot n)\right\|_{H^{4-j}\left(\mathbb{R}^{2}\right)}^{2}+\sum_{0 \leqq j \leqq 3} \int_{0}^{t}\left\|\partial_{t}^{j}(u \cdot n)\right\|_{H^{5-j}\left(\mathbb{R}^{2}\right)}^{2} \mathrm{~d} \tau \text {. }
\end{aligned}
$$

Proposition 30. The velocity $u$ satisfies the following energy estimate.

$$
\begin{aligned}
\mathcal{E}_{L}(u)(t) \leqq & \frac{1}{\varepsilon} M_{\mu}^{0}+\frac{1}{\varepsilon} K_{\mu}(t)\left(t+\int_{0}^{t} \mathcal{E}_{L}(\tau)+\overline{\mathcal{E}}_{L}(\tau)+\mathcal{E}_{L}(\omega)(\tau) \mathrm{d} \tau\right) \\
& +\varepsilon\left(M_{\mu}^{0}+t K_{\mu}(t)\right)\left(\mathcal{E}_{L}(t)+\overline{\mathcal{E}}_{L}(t)+\mathcal{E}_{L}(\omega)(t)\right)
\end{aligned}
$$

for $t \in(0, T)$, where we are free to choose $\varepsilon>0$.

Proof. See the rest of this section for the proof

We use the weak form of the equations to avoid the difficulty of not having enough regularity on $u$. As in 12.3 , we set

$$
\stackrel{\curvearrowright}{u}=N^{-1} u
$$

where the matrix $N$ is defined in Definition 36. We define the energy associated with $\tilde{u}$ as

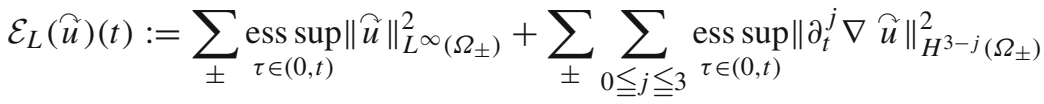

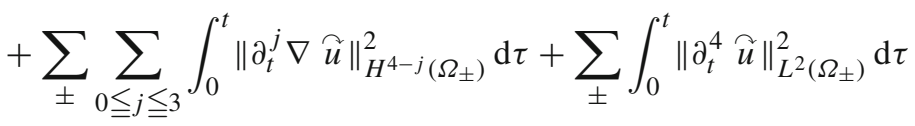

$$
\begin{aligned}
& +\sum_{0 \leqq j \leqq 3} \operatorname{ess} \sup \left\|\partial_{t}^{j} \widetilde{u}_{3}\right\|_{H^{4-j}\left(\mathbb{R}^{2}\right)}^{2}+\sum_{0 \leqq j \leqq 3} \int_{0}^{t}\left\|\partial_{t}^{j} \widetilde{u}_{3}\right\|_{H^{5-j}\left(\mathbb{R}^{2}\right)}^{2} \mathrm{~d} \tau \text {. }
\end{aligned}
$$

Lemma 24. We claim that

$$
\mathcal{E}_{L}(u)(t) \leqq\left(M_{\mu}^{0}+t K_{\mu}(t)\right)\left(\mathcal{E}_{L}(\widetilde{u})(t)+\mathcal{E}_{L}(f)(t)\right)
$$

and conversely

$$
\mathcal{E}_{L}\left(\stackrel{\widetilde{u}}{)}(t) \leqq\left(M_{\mu}^{0}+t K_{\mu}(t)\right)\left(\mathcal{E}_{L}(u)(t)+\mathcal{E}_{L}(f)(t)\right) .\right.
$$


Proof. This is straightforward using the Sobolev embedding theorem and the lifting property in the definition of $N$. We recall that $\widetilde{\sim}_{3}=\left(N^{-1} u\right)_{3}=u \cdot n$ so the estimate of the boundary terms is immediate.

We recall that $\tilde{u}$ satisfies the weak equation (154). Let $0 \leqq|\alpha| \leqq 3$ with $\alpha_{3}=0$ be a multi-index. We may also write $\partial^{\alpha}=\partial_{t}^{j} \nabla^{\beta}$ where $\beta_{3}=0$ and $0 \leqq j+|\beta| \leqq 3$. We apply $\partial_{t}^{j}$ to the weak equation (154), then test it with $(-1)^{|\beta|} \nabla^{\beta} w$ for $w \in B^{1}$ smooth in the horizontal directions, then integrate by parts horizontally and use the density of such $w$ in $B^{1}$ to obtain that $\partial^{\alpha} \stackrel{\curvearrowright}{u}$ satisfies the following equation:

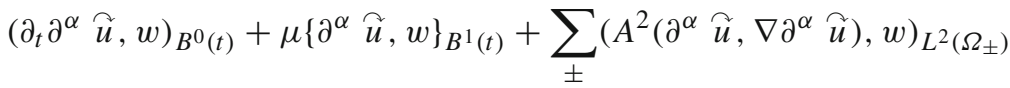

$$
\begin{aligned}
& =\sum_{ \pm}\left(G^{1, \alpha}, w\right)_{L^{2}\left(\Omega_{ \pm}\right)}+\sum_{ \pm}\left(G^{2, \alpha}, \nabla w\right)_{L^{2}\left(\Omega_{ \pm}\right)} \\
& +\left(G^{3, \alpha}, w_{3}\right)_{L^{2}(\Gamma)}+\left(G^{4, \alpha}, \nabla^{\prime} w_{3}\right)_{L^{2}(\Gamma)}
\end{aligned}
$$

for all $w \in B^{1}$ and almost every $t \in(0, T)$, where

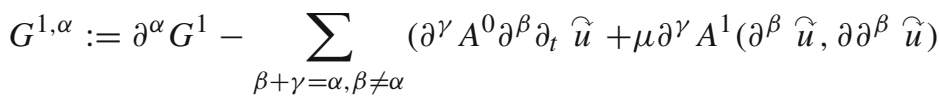

$$
\begin{aligned}
& \left.+\partial^{\gamma} A^{2}\left(\partial^{\beta} \stackrel{\curvearrowright}{u}, \nabla \partial^{\beta} \stackrel{\curvearrowright}{u}\right)\right) \\
& G^{2, \alpha}:=\partial^{\alpha} G^{2}-\mu \sum_{\beta+\gamma=\alpha, \beta \neq \alpha} \partial^{\gamma} A^{1}\left(\partial^{\beta} \stackrel{\curvearrowright}{u}, \nabla \partial^{\beta} \stackrel{\widetilde{u}}{)}\right. \\
& G^{3, \alpha}:=\partial^{\alpha} G^{3} \\
& G^{4, \alpha}:=\partial^{\alpha} G^{4}
\end{aligned}
$$

and $A^{i}$ for $0 \leqq i \leqq 2$ are defined in Definition 42 and $G^{i}$ for $1 \leqq i \leqq 4$ are defined in Definition 43.

Lemma 25. We claim that the constant $C_{\infty, 0}(t)$ and $C_{\infty, 1}(t)$, as defined in Definition 35, satisfy the following estimates:

$$
\begin{aligned}
& C_{\infty, 0}(t) \leqq M_{\mu}^{0}+t K_{\mu}(t) \\
& C_{\infty, 1}(t) \leqq K_{\mu}(t) .
\end{aligned}
$$

Proof. This is straightforward using the definition of $C_{\infty, j}(t)$ and the Sobolev embedding theorem.

Lemma 26. Let $1 \leqq|\alpha| \leqq 3$. We claim that

$$
\begin{aligned}
\int_{0}^{t}\left\|G^{1, \alpha}\right\|_{L^{2}\left(\Omega_{ \pm}\right)}^{2} \mathrm{~d} \tau & \leqq K_{\mu}(t)\left(t+\int_{0}^{t} \mathcal{E}_{L}(\tau)+\overline{\mathcal{E}}_{L}(\tau)+\mathcal{E}_{L}(\omega)(\tau) \mathrm{d} \tau\right) \\
\int_{0}^{t}\left\|\partial_{t} G^{2, \alpha}\right\|_{L^{2}\left(\Omega_{ \pm}\right)}^{2} \mathrm{~d} \tau & \leqq\left(M_{\mu}^{0}+t K_{\mu}(t)\right)\left(1+\overline{\mathcal{E}}_{L}(t)+\mathcal{E}_{L}(t)+\mathcal{E}_{L}(\omega)(t)\right) \\
\int_{0}^{t}\left\|G^{2, \alpha}\right\|_{H^{1}\left(\Omega_{ \pm}\right)}^{2} \mathrm{~d} \tau & \leqq K_{\mu}(t)\left(t+\int_{0}^{t} \overline{\mathcal{E}}_{L}(\tau)+\mathcal{E}_{L}(\tau)+\mathcal{E}_{L}(\omega)(\tau) \mathrm{d} \tau\right)
\end{aligned}
$$




$$
\begin{aligned}
& \sum_{k=0}^{1} \int_{0}^{t}\left\|\partial^{k} G^{3, \alpha}\right\|_{L^{2}(\Gamma)}^{2} \mathrm{~d} \tau \leqq t K_{\mu}(t) \\
& \sum_{k=0}^{1} \int_{0}^{t}\left\|\partial^{k} G^{4, \alpha}\right\|_{L^{2}(\Gamma)}^{2} \mathrm{~d} \tau \leqq K_{\mu}(t) \int_{0}^{t} \overline{\mathcal{E}}_{L}(\tau)+\mathcal{E}_{L}(\tau) \mathrm{d} \tau
\end{aligned}
$$

Furthermore, for $2 \leqq i \leqq 4$,

$$
\begin{aligned}
\underset{\tau \in(0, t)}{\operatorname{ess} \sup \left\|G^{i, \alpha}\right\|_{L^{2}\left(\Omega_{ \pm}\right)}^{2} \leqq} & M_{\mu}^{0}+\frac{1}{\varepsilon} K_{\mu}(t)\left(t+\int_{0}^{t} \overline{\mathcal{E}}_{L}(t)+\mathcal{E}_{L}(\tau)+\mathcal{E}_{L}(\omega)(\tau) \mathrm{d} \tau\right) \\
& +\varepsilon\left(M_{\mu}^{0}+t K_{\mu}(t)\right)\left(\mathcal{E}_{L}(t)+\overline{\mathcal{E}}_{L}(t)+\mathcal{E}_{L}(\omega)(t)\right) .
\end{aligned}
$$

Proof. This is a straightforward, if long and technical, computation using the Sobolev embedding theorem and the definition of the energy $\mathcal{E}_{L}(t)$ and $\mathcal{E}_{L}(\omega)(t)$. Recall that our estimate of $\partial_{t}^{4} \omega$ is only $L^{2}$ not $L^{\infty}$ in time and hence the estimate for $\partial_{t} G^{2, \alpha}$ is not as good as the other estimates.

Now we apply the energy estimate (149) to the equation for $\partial^{\alpha} \stackrel{\mathcal{u}}{\text {, where }}$ $1 \leqq|\alpha| \leqq 3$ and $\alpha_{3}=0$ to obtain the following estimate:

$$
\begin{aligned}
& \int_{0}^{t}\left\|\partial_{t} \partial^{\alpha} \stackrel{\curvearrowright}{u}\right\|_{B^{0}(\tau)}^{2} \mathrm{~d} \tau+\underset{\tau \in(0, t)}{\operatorname{ess} \sup \left\|\partial^{\alpha} \stackrel{\curvearrowright}{u}\right\|_{B^{1}(t)}^{2} \leqq} \frac{C_{\infty, 1}}{\varepsilon} t\left\|\partial_{0}^{\mu, g, j} \nabla^{\beta} \stackrel{\overbrace{}}{u}^{0}\right\|_{B^{0}}^{2} \\
& +\frac{C_{\infty, 1}}{\varepsilon} \int_{0}^{t} \sum_{ \pm}\left\|G^{1, \alpha}\right\|_{L^{2}\left(\Omega_{ \pm}\right)}^{2}+\sum_{ \pm}\left\|G^{2, \alpha}\right\|_{L^{2}\left(\Omega_{ \pm}\right)}^{2}+\left\|G^{3, \alpha}\right\|_{L^{2}(\Gamma)}^{2} \\
& +\left\|G^{4, \alpha}\right\|_{L^{2}(\Gamma)}^{2} \mathrm{~d} \tau \\
& +C_{\infty, 0}\left(\left\|\partial_{0}^{\mu, g, j} \nabla^{\beta} \stackrel{\curvearrowright}{u}\right\|_{B^{1}}^{2}+\underset{\tau \in(0, t)}{\operatorname{ess~sup}}\left(\sum_{ \pm}\left\|G^{2, \alpha}\right\|_{L^{2}\left(\Omega_{ \pm}\right)}^{2}+\left\|G^{3, \alpha}\right\|_{L^{2}(\Gamma)}^{2}\right.\right. \\
& \left.\left.\quad+\left\|G^{4, \alpha}\right\|_{L^{2}(\Gamma)}^{2}\right)\right) \\
& +\varepsilon \int_{0}^{t} \sum_{ \pm}\left\|\partial_{t} G^{2, \alpha}\right\|_{L^{2}\left(\Omega_{ \pm}\right)}^{2}+\left\|\partial_{t} G^{3, \alpha}\right\|_{L^{2}(\Gamma)}^{2}+\left\|\partial_{t} G^{4, \alpha}\right\|_{L^{2}(\Gamma)}^{2} \mathrm{~d} \tau .
\end{aligned}
$$

To estimate the right hand side we use the estimate 25 for $C_{\infty, 0}$ and $C_{\infty, 1}$ and the estimate 26 for the $G^{i, \alpha}$, thus we obtain:

$$
\begin{aligned}
& \int_{0}^{t}\left\|\partial_{t} \partial^{\alpha} \stackrel{\curvearrowright}{u}\right\|_{B^{0}(\tau)}^{2} \mathrm{~d} \tau+\underset{\tau \in(0, t)}{\operatorname{ess} \sup \left\|\partial^{\alpha} \stackrel{\curvearrowright}{u}\right\|_{B^{1}(t)}^{2}} \\
& \leqq M_{\mu}^{0}+\frac{1}{\varepsilon} K_{\mu}(t)\left(t+\int_{0}^{t} \mathcal{E}_{L}(\tau)+\overline{\mathcal{E}}_{L}(\tau)+\mathcal{E}_{L}(\omega)(\tau) \mathrm{d} \tau\right) \\
& \quad+\varepsilon\left(M_{\mu}^{0}+t K_{\mu}(t)\right)\left(\mathcal{E}_{L}(t)+\overline{\mathcal{E}}_{L}(t)+\mathcal{E}_{L}(\omega)(t)\right) .
\end{aligned}
$$


Now to estimate the highest order space derivative, we apply the energy estimate (153) to the equation for $\partial^{\alpha} \stackrel{\curvearrowright}{u}=\partial_{t}^{j} \nabla^{\beta}$, where $1 \leqq|\alpha| \leqq 3$ and $\alpha_{3}=0$. We obtain:

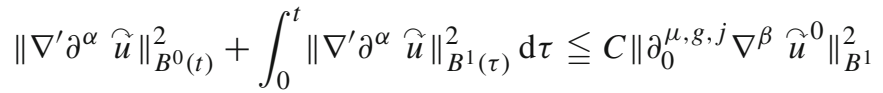

$$
\begin{aligned}
& +C_{\infty, 1} \int_{0}^{t} \sum_{ \pm}\left\|G^{1, \alpha}\right\|_{L^{2}\left(\Omega_{ \pm}\right)}^{2}+\sum_{ \pm}\left\|G^{2, \alpha}\right\|_{H^{1}\left(\Omega_{ \pm}\right)}^{2} \mathrm{~d} \tau+\left\|G^{3, \alpha}\right\|_{L^{2}(\Gamma)}^{2} \\
& +\left\|G^{4, \alpha}\right\|_{H^{1}(\Gamma)}^{2} \mathrm{~d} \tau \\
& +\frac{C_{\infty, 1}}{\varepsilon} \int_{0}^{t}\left\|\partial^{\alpha} \stackrel{\curvearrowright}{\tilde{u}}\right\|_{H^{1}\left(\Omega_{ \pm}\right)}^{2} \mathrm{~d} \tau+\varepsilon \int_{0}^{t}\left\|\partial_{t} \partial^{\alpha} \stackrel{\curvearrowright}{u}\right\|_{L^{2}\left(\Omega_{ \pm}\right)}^{2} \mathrm{~d} \tau .
\end{aligned}
$$

Applying the estimates 25 and 26 to the right hand side, we obtain:

$$
\begin{aligned}
& \left\|\nabla^{\prime} \partial^{\alpha} \stackrel{\curvearrowright}{u}\right\|_{B^{0}(t)}^{2}+\int_{0}^{t}\left\|\nabla^{\prime} \partial^{\alpha} \stackrel{\curvearrowright}{u}\right\|_{B^{1}(\tau)}^{2} \mathrm{~d} \tau \\
& \leqq M_{\mu}^{0}+\frac{1}{\varepsilon} K_{\mu}(t)\left(t+\int_{0}^{t} \mathcal{E}_{L}(\tau)+\overline{\mathcal{E}}_{L}(\tau)+\mathcal{E}_{L}(\omega)(\tau) \mathrm{d} \tau\right) \\
& \quad+\varepsilon\left(M_{\mu}^{0}+t K_{\mu}(t)\right) \mathcal{E}_{L}(t) .
\end{aligned}
$$

It remains to estimate the highest order normal derivatives. However, this is quite straightforward, if technical, because we may rearrange the rotated velocity equation to obtain an expression for $\partial_{3}^{2} \hat{u}$ in terms of quantities with fewer normal derivatives or existing estimates then use these estimates together with an induction on $\alpha_{3}$ for $0 \leqq \alpha_{3} \leqq 3$ to obtain the estimate:

$$
\begin{aligned}
\sum_{0 \leqq|\alpha| \leqq 3} \int_{0}^{t}\left\|\partial^{\alpha} \stackrel{\Im}{u}\right\|_{H^{2}\left(\Omega_{ \pm}\right)}^{2} \mathrm{~d} \tau \leqq & \frac{1}{\varepsilon} M_{\mu}^{0}+\frac{1}{\varepsilon} K_{\mu}(t)\left(t+\int_{0}^{t} \mathcal{E}_{L}(\tau)+\overline{\mathcal{E}}_{L}(\tau)\right. \\
& \left.+\mathcal{E}_{L}(\omega)(\tau) \mathrm{d} \tau\right) \\
& +\varepsilon\left(M_{\mu}^{0}+t K_{\mu}(t)\right)\left(\mathcal{E}_{L}(t)+\overline{\mathcal{E}}_{L}(t)+\mathcal{E}_{L}(\omega)(t)\right) .
\end{aligned}
$$

Combining all the above estimates, we have shown that

$$
\begin{aligned}
\mathcal{E}_{L}(\stackrel{\curvearrowright}{u})(t) \leqq & \frac{1}{\varepsilon} M_{\mu}^{0}+\frac{1}{\varepsilon} K_{\mu}(t)\left(t+\int_{0}^{t} \mathcal{E}_{L}(\tau)+\overline{\mathcal{E}}_{L}(\tau)+\mathcal{E}_{L}(\omega)(\tau) \mathrm{d} \tau\right) \\
& +\varepsilon\left(M_{\mu}^{0}+t K_{\mu}(t)\right)\left(\mathcal{E}_{L}(t)+\overline{\mathcal{E}}_{L}(t)+\mathcal{E}_{L}(\omega)(t)\right)
\end{aligned}
$$

Now using the estimate (174) and the estimate (159) for the front, we obtain

$$
\begin{aligned}
\mathcal{E}_{L}(u)(t) \leqq & \frac{1}{\varepsilon} M_{\mu}^{0}+\frac{1}{\varepsilon} K_{\mu}(t)\left(t+\int_{0}^{t} \mathcal{E}_{L}(\tau)+\overline{\mathcal{E}}_{L}(\tau)+\mathcal{E}_{L}(\omega)(\tau) \mathrm{d} \tau\right) \\
& +\varepsilon\left(M_{\mu}^{0}+t K_{\mu}(t)\right)\left(\mathcal{E}_{L}(t)+\overline{\mathcal{E}}_{L}(t)+\mathcal{E}_{L}(\omega)(t)\right)
\end{aligned}
$$

This completes the proof of the estimate of the velocity, (173). 
Proposition 31. The normal component of the velocity, $u \cdot n$, satisfies the following additional energy estimate.

$$
\begin{aligned}
\sum_{j=0}^{3} \int_{0}^{t}\left\|\partial_{t}^{j}(u \cdot n)\right\|_{H^{5.5-j}(\Gamma)}^{2} \mathrm{~d} \tau \leqq & \frac{M_{\mu}^{0}}{\varepsilon}+\frac{1}{\varepsilon} K_{\mu}(t)\left(t+\int_{0}^{t} \mathcal{E}_{L}(\tau)+\overline{\mathcal{E}}_{L}(\tau)\right. \\
& \left.+\mathcal{E}_{L}(\omega)(\tau) \mathrm{d} \tau\right) \\
& +\varepsilon\left(M_{\mu}^{0}+t K_{\mu}(t)\right)\left(\mathcal{E}_{L}(t)+\overline{\mathcal{E}}_{L}(t)+\mathcal{E}_{L}(\omega)(t)\right)
\end{aligned}
$$

for $t \in(0, T)$.

Proof. Let $0 \leqq j \leqq 3$. Applying $\partial_{t}^{j}$ to the pressure interface condition (134) we have

$$
\left(1-\Delta^{\prime}\right) \partial_{t}^{j}(u \cdot n)=\partial_{t}^{j}\left(\left[\rho c^{2} \nabla^{\psi} \cdot u-\frac{1}{\mu} p\right]-\frac{1}{\mu} \sigma \nabla^{\prime} \cdot \hat{n}+\frac{1}{\mu} g_{2}\right) .
$$

We apply Lemma 11 with

$$
\begin{aligned}
& z=\partial_{t}^{j}(u \cdot n) \\
& h=\partial_{t}^{j}\left(\left[\rho c^{2} \nabla^{\psi} \cdot u-\frac{1}{\mu} p\right]-\frac{1}{\mu} \sigma \nabla^{\prime} \cdot \hat{n}+\frac{1}{\mu} g_{2}\right)
\end{aligned}
$$

to conclude that

$$
\begin{aligned}
& \left\|\partial_{t}^{j}(u \cdot n)\right\|_{H^{5.5-j}(\Gamma)}^{2} \\
& \quad \leqq C\left\|\partial_{t}^{j}\left(\left[\rho c^{2} \nabla^{\psi} \cdot u-\frac{1}{\mu} p\right]-\frac{1}{\mu} \sigma \nabla^{\prime} \cdot \hat{n}+\frac{1}{\mu} g_{2}\right)\right\|_{H^{3.5-j}(\Gamma)}^{2} .
\end{aligned}
$$

We conclude using the chain rule, Sobolev embedding and existing estimates.

\subsection{Conclusion of the Energy Estimate}

Putting together what we have so far we have shown for $t \in(0, T)$ that

$$
\begin{aligned}
\mathcal{E}_{L}(t)+\mathcal{E}_{L}(\omega)(t) \leqq & \frac{1}{\varepsilon} M_{\mu}^{0}+\frac{1}{\varepsilon} K_{\mu}(t)\left(t+\int_{0}^{t} \mathcal{E}_{L}(\tau)+\overline{\mathcal{E}}_{L}(\tau)+\mathcal{E}_{L}(\omega)(\tau) \mathrm{d} \tau\right) \\
& +\varepsilon\left(M_{\mu}^{0}+t K_{\mu}(t)\right)\left(\mathcal{E}_{L}(t)+\overline{\mathcal{E}}_{L}(t)+\mathcal{E}_{L}(\omega)(t)\right) .
\end{aligned}
$$

Moreover from (162), (168), and (169), we have

$$
\rho \geqq \delta^{0}-t K_{\mu}(t), \quad \rho_{\mu} \geqq \delta_{\mu}^{0}-t K_{\mu}(t), \quad J^{\psi} \geqq \kappa^{0}-t K_{\mu}(t) .
$$

Choosing $\varepsilon=\frac{1}{5\left(M_{\mu}^{0}+t K_{\mu}(t)\right)}$, we obtain

$$
\begin{aligned}
\mathcal{E}_{L}(t)+\mathcal{E}_{L}(\omega)(t) \leqq & M_{\mu}^{0}+K_{\mu}(t)\left(t+\int_{0}^{t} \overline{\mathcal{E}}_{L}(\tau)+\mathcal{E}_{L}(\tau)+\mathcal{E}_{L}(\omega)(\tau) \mathrm{d} \tau\right) \\
& +\frac{1}{5}\left(\overline{\mathcal{E}}_{L}(t)+\mathcal{E}_{L}(t)+\mathcal{E}_{L}(\omega)(t)\right)
\end{aligned}
$$


for $t \in(0, T)$ where we have redefined $M_{\mu}^{0}$ and $K_{\mu}(t)$. Rearranging, we obtain:

$$
\begin{aligned}
\mathcal{E}_{L}(t)+\mathcal{E}_{L}(\omega)(t) \leqq & M_{\mu}^{0}+K_{\mu}(t)\left(t+\int_{0}^{t} \overline{\mathcal{E}}_{L}(\tau)+\mathcal{E}_{L}(\tau)+\mathcal{E}_{L}(\omega)(\tau) \mathrm{d} \tau\right) \\
& +\frac{1}{4} \overline{\mathcal{E}}_{L}(t) .
\end{aligned}
$$

This completes the proof of Proposition 11.

\section{The Fixed Point Scheme}

Here we intend to prove the existence of a solution to the $\mu$-approximate equations for fixed $\mu$ using the contraction mapping theorem.

\subsection{The Functional Framework}

In this section we assume we are given initial data $\left(U_{+}^{0}, U_{-}^{0}, f^{0}\right)$ for the $\mu$ approximate equations with $\mathcal{E}_{\mu}^{0}<\infty$ which satisfies the compatibility condition (45) up to order 3 and the compatibility condition (46) up to order 2 and

$$
\inf _{\Omega_{ \pm}} \rho^{0}=: \delta^{0}>0, \quad \inf _{\Omega_{ \pm}} \rho_{\mu}^{0}=: \delta_{\mu}^{0}>0, \quad \inf _{\mathbb{R}^{3}} J^{\psi^{0}}=: \kappa^{0}>0 .
$$

Definition 45. We define the set $Y$ as follows:

$$
\begin{aligned}
Y & =\left\{\left(U_{+}, U_{-}, f\right): U_{ \pm} \in L^{\infty}\left((0, T) \times \Omega_{ \pm}\right), f \in L^{\infty}((0, T) \times \Gamma) \text { with } \mathcal{E}_{L}(T)\right. \\
& \left.\leqq R^{0},\left.\partial_{t}^{j}\left(U_{+}, U_{-}, f\right)\right|_{t=0}=\partial_{0}^{\mu, g, j}\left(U_{+}^{0}, U_{-}^{0}, f^{0}\right) \text { for } 0 \leqq j \leqq 3,[u \cdot n]=0\right\},
\end{aligned}
$$

where $R^{0}<\infty$ is a constant which will be fixed later, sufficiently large depending the initial data, $T \in(0,1]$ will be fixed later, sufficiently small depending on the initial data, and $\mathcal{E}_{L}(t)$ is the energy associated to $\left(U_{+}, U_{-}, f\right)$ as defined in Definition 22. Note if sufficiently many weak derivatives do not exist to define $\mathcal{E}_{L}(t)$ then we adopt the convention $\mathcal{E}_{L}(t)=\infty$.

Definition 46. We define the Banach space $Z$ as follows:

$$
\begin{aligned}
Z= & \left\{\left(U_{+}, U_{-}, f, w\right): U_{ \pm} \in L_{\mathrm{loc}}^{1}\left((0, T) \times \Omega_{ \pm}\right), f, w \in L_{\mathrm{loc}}^{1}((0, T) \times \Gamma)\right. \\
& \text { with } \left.\left\|\left(U_{+}, U_{-}, f, w\right)\right\|_{Z}<\infty\right\},
\end{aligned}
$$

where

$$
\left\|\left(U_{+}, U_{-}, f, w\right)\right\|_{Z}:=\left\|\left(U_{+}, U_{-}, f, w\right)\right\|_{Z(T)},
$$

and for each $t \in(0, T]$, we define

$$
\left\|\left(U_{+}, U_{-}, f, w\right)\right\|_{Z(t)}^{2}=\sum_{ \pm} \sum_{0 \leqq j \leqq 1} \operatorname{ess} \sup \left\|\partial_{t}^{j} U\right\|_{H^{2-j}\left(\Omega_{ \pm}\right)}^{2}
$$




$$
\begin{aligned}
& +\sum_{ \pm} \int_{0}^{t}\left\|\partial_{t}^{2} U\right\|_{L^{2}\left(\Omega_{ \pm}\right)}^{2} \mathrm{~d} \tau \\
& +\sum_{ \pm} \sum_{0 \leqq j \leqq 1} \int_{0}^{t}\left\|\partial_{t}^{j} u\right\|_{H^{3-j}\left(\Omega_{ \pm}\right)}^{2} \mathrm{~d} \tau+\sum_{0 \leqq j \leqq 1} \underset{\tau \in(0, t)}{\operatorname{ess~sup}\|f\|_{H^{3.5-j}\left(\mathbb{R}^{2}\right)}^{2}} \\
& +\int_{0}^{t}\left\|\partial_{t}^{2} f\right\|_{H^{1}(\Gamma)}^{2} \mathrm{~d} \tau \\
& +\sum_{0 \leqq j \leqq 1} \underset{\tau \in(0, t)}{\operatorname{ess~sup}\left\|\partial_{t}^{j} w\right\|_{H^{2-j}(\Gamma)}^{2}+\sum_{0 \leqq j \leqq 1} \int_{0}^{t}\left\|\partial_{t}^{j} w\right\|_{H^{3.5-j}(\Gamma)}^{2} \mathrm{~d} \tau .}
\end{aligned}
$$

We define the metric $d_{Y}$ on $Y$ as follows:

$$
d_{Y}\left(\left(U_{+}, U_{-}, f\right),\left(\tilde{U}_{+}, \tilde{U}_{-}, \tilde{f}\right)\right):=\left\|\left(U_{+}, U_{-}, f, u \cdot n\right)-\left(\tilde{U}_{+}, \tilde{U}_{-}, \tilde{f}, \tilde{u} \cdot \tilde{n}\right)\right\|_{Z} \cdot
$$

Clearly $Z$ is a Banach space with norm $\|\cdot\|_{Z}$, and if $\left(U_{+}, U_{-}, f\right) \in Y$ then we have $\left(U_{+}, U_{-}, f, u \cdot n\right)-\left(U_{+}^{0}, U_{-}^{0}, f^{0}, u^{0} \cdot n^{0}\right) \in Z$.

Note that $\|\cdot\|_{Z(t)}$ is defined so that $\left\|\left(U_{+}, U_{-}, f, u \cdot n\right)\right\|_{Z(t)}^{2}$ is effectively equal to the linearised energy $\mathcal{E}_{L}$ but two orders of derivatives lower and with $L^{2}$-in-space instead of $L^{\infty}$-in-space norms at zero order.

Proposition 32. $Y$ is a complete metric space with respect to the metric $d_{Y}$.

Proof. This is straightforward using weak-* compactness and Sobolev compact embedding on bounded domains.

\subsection{The Contraction Mapping}

Proposition 33. Given a background state $\left(\bar{U}_{+}, \bar{U}_{-}, \bar{f}\right) \in Y$, there exists a solution $\left(U_{+}, U_{-}, f\right) \in Y$ of the linearised equations with background state $\left(\bar{U}_{+}, \bar{U}_{-}, \bar{f}\right)$ assuming we have chosen $T>0$ sufficiently small and $R^{0}$ sufficiently large depending on the size of the initial data. In fact $T$ and $R^{0}$ should satisfy the following inequalities:

$$
\begin{aligned}
R^{0} & \geqq R_{\mu}\left(\mathcal{E}_{\mu}^{0}, \frac{1}{\delta^{0}}, \frac{1}{\delta_{\mu}^{0}}, \frac{1}{\kappa^{0}}, E_{g}(1)\right) \\
T & \leqq T_{\mu}\left(R^{0}, \mathcal{E}_{\mu}^{0}, \frac{1}{\delta^{0}}, \frac{1}{\delta_{\mu}^{0}}, \frac{1}{\kappa^{0}}, E_{g}(1)\right),
\end{aligned}
$$

where $R_{\mu}$ is a smooth increasing function and $T_{\mu}$ is a smooth decreasing function. Moreover, if $\omega$ is the corresponding solution to the linearised curl equation (130), we may assume

$$
\mathcal{E}_{L}(T)+\mathcal{E}_{L}(\omega)(T) \leqq R^{0}
$$


Proof. This is straightforward using the existence of a solution to the linearised equations given by Proposition 12 and the maximal time limiting criteria therein, together with the energy estimate and lower bound estimates for the linearised equations given in Proposition 11.

Proposition 34. For $i=1,2$, let $\left(U_{+}^{i}, U_{-}^{i}, f^{i}\right) \in Y$ be solutions of the linearised equations with background states $\left(\bar{U}_{+}^{i}, \bar{U}_{-}^{i}, \bar{f}^{i}\right) \in Y$, as described in Definition 32. Then

$$
d_{Y}\left(\left(U_{+}^{1}, U_{-}^{1}, f^{1}\right),\left(U_{+}^{2}, U_{-}^{2}, f^{2}\right)\right) \leqq \lambda d_{Y}\left(\left(\bar{U}_{+}^{1}, \bar{U}_{-}^{1}, \bar{f}^{1}\right),\left(\bar{U}_{+}^{2}, \bar{U}_{-}^{2}, \bar{f}^{2}\right)\right)
$$

for some $\lambda<1$ which is fixed independent of the solutions and background states, provided we have chosen $T>0$ sufficiently small and $R^{0}$ sufficiently large depending on the size of the initial data. In fact $T$ and $R^{0}$ should satisfy the following inequalities:

$$
\begin{array}{r}
R^{0} \geqq \tilde{R}_{\mu}\left(\mathcal{E}_{\mu}^{0}, \frac{1}{\delta^{0}}, \frac{1}{\delta_{\mu}^{0}}, \frac{1}{\kappa^{0}}, E_{g}(1)\right) \\
T \leqq \tilde{T}_{\mu}\left(R^{0}, \mathcal{E}_{\mu}^{0}, \frac{1}{\delta^{0}}, \frac{1}{\delta_{\mu}^{0}}, \frac{1}{\kappa^{0}}, E_{g}(1)\right),
\end{array}
$$

where $\tilde{R}_{\mu}$ is a smooth increasing function and $\tilde{T}_{\mu}$ is a smooth decreasing function.

Proof. Note that the proof is very similar to that of the linearised energy estimate proved in Section 13, replacing the energy $\mathcal{E}_{L}$ with $\mathcal{E}_{\Delta}:=d_{Y}\left(\left(U_{+}^{1}, U_{-}^{1}, f^{1}\right)\right.$, $\left.\left(U_{+}^{2}, U_{-}^{2}, f^{2}\right)\right)$ and taking the difference of the equations for the two solutions after differentiating. Note we are working two orders of regularity lower than the energy estimate so we do not need to be so careful about estimating coefficients. We write terms of the form $G\left(\bar{U}^{1}, \bar{\psi}^{1}\right) \partial^{\alpha} \partial U^{1}-G\left(\bar{U}^{2}, \bar{\psi}^{2}\right) \partial^{\alpha} \partial U^{2}$ as $G\left(\bar{U}^{1}, \bar{\psi}^{1}\right) \partial^{\alpha} \partial\left(U^{1}-U^{2}\right)+\left(G\left(\bar{U}^{1}, \bar{\psi}^{1}\right)-G\left(\bar{U}^{2}, \bar{\psi}^{2}\right)\right) \partial^{\alpha} \partial U^{2}$ and estimate the first term as in the linearised energy estimate and the second term using the mean value theorem.

Following this strategy, we obtain, after an application of Gronwall's lemma,

$$
\mathcal{E}_{\Delta}(t) \leqq\left(\mathcal{K}_{L}(t) \int_{0}^{t} \overline{\mathcal{E}}_{\Delta}(\tau) \mathrm{d} \tau+\frac{1}{4} \overline{\mathcal{E}}_{\Delta}(t)\right) \exp \left(t \mathcal{K}_{L}(t)\right)
$$

for $t \in(0, T)$, where we have modified the definition of $\mathcal{K}_{L}(t)$ slightly to include the energies $\mathcal{E}_{L}^{i}(t)$ of both $\left(U_{+}^{1}, U_{-}^{1}, f^{1}\right)$ and $\left(U_{+}^{2}, U_{-}^{2}, f^{2}\right)$ and the energies $\overline{\mathcal{E}}_{L}^{i}(t)$ of their background states and the energies $\mathcal{E}_{L}\left(\omega^{i}\right)(t)$ of their associated curls.

Assuming $R^{0}$ and $T$ satisfy the inequalities given in Proposition 33, we have

$$
\mathcal{K}_{L}(T) \leqq F_{\mu}\left(R^{0}, M_{\mu}^{0}\right)
$$

for some smooth increasing function $F_{\mu}$ which may depend on $\mu$. Thus choosing $T$ sufficiently small depending on $R^{0}$ and $M_{\mu}^{0}$ we may ensure

$$
\mathcal{E}_{\Delta}(T) \leqq \frac{3}{4} \overline{\mathcal{E}}_{\Delta}(T)
$$

which concludes the proof. 
Proposition 35. Define the map $\Phi: Y \rightarrow Y$ by $\left(\bar{U}_{+}, \bar{U}_{-}, \bar{f}\right) \mapsto\left(U_{+}, U_{-}, f\right)$ where $\left(U_{+}, U_{-}, f\right)$ is the unique solution of the linearised equations with background state $\left(\bar{U}_{+}, \bar{U}_{-}, \bar{f}\right)$ as given by Proposition 12 . Assume that $R^{0}$ and $T$ satisfy the inequalities in Propositions 33 and 34. Assume also that the space $Y$ is nonempty. Then $\Phi$ has a fixed point.

Proof. By Proposition 12 we know that

$$
\begin{aligned}
\mathcal{E}_{L}(T) & <\infty \\
\left.\partial_{t}^{j}\left(U_{+}, U_{-}, f\right)\right|_{t=0} & =\partial_{0}^{\mu, g, j}\left(U_{+}^{0}, U_{-}^{0}, f^{0}\right) \text { for } 0 \leqq j \leqq 3 . \\
{[u \cdot n] } & =0
\end{aligned}
$$

By Proposition 33 we have

$$
\mathcal{E}_{L}(T) \leqq R^{0},
$$

and hence $\left(U_{+}, U_{-}, f\right) \in Y$. Now Proposition 34 implies that the map $\Phi: Y \rightarrow$ $Y$ is a contraction. Since $Y$ is complete, we may apply the contraction mapping theorem to conclude that $\Phi$ has a unique fixed point in $Y$.

\subsection{The Existence of a Solution to the $\mu$-Approximate Equations}

Lemma 27. Suppose $\left(U_{+}, U_{-}, f\right)$ is a fixed point of the linearised equations on the time interval $(0, T)$, by which we mean $\left(U_{+}, U_{-}, f\right)$ together with $\left(\omega_{+}, \omega_{-}\right)$ is a solution of the linearised equations on the time interval $(0, T)$ as defined in Definition 32, linearised about the state $\left(U_{+}, U_{-}, f\right)$, with initial value $\left.\left(U_{+}, U_{-}, f\right)\right|_{t=0}=\left(U_{+}^{0}, U_{-}^{0}, f^{0}\right)$. Suppose also that

$$
\mathcal{E}_{L}(T)<\infty, \quad \rho \geqq \frac{\delta^{0}}{4}, \quad \rho_{\mu} \geqq \frac{\delta_{\mu}^{0}}{4}, \quad J^{\psi} \geqq \frac{1}{4} \kappa^{0},
$$

where $\mathcal{E}_{L}(t)$ is the linearised energy associated with $\left(U_{+}, U_{-}, f\right)$. Then $\left(U_{+}, U_{-}, f\right)$ is a solution of the $\mu$-approximate equations on the time interval $(0, T)$ with initial $\operatorname{data}\left(U_{+}^{0}, U_{-}^{0}, f^{0}\right)$.

Moreover, there exists $T_{\mu}^{0} \in(0, T]$ and $C_{\mu}^{0}>0$ such that

$$
\begin{aligned}
\mathcal{E}_{\mu}\left(\min \left\{T_{\mu}^{0}, T\right\}\right) & \leqq C_{\mu}^{0}<\infty \\
\rho & \geqq \frac{\delta^{0}}{2} \quad \text { in } \quad\left(0, \min \left\{T_{\mu}^{0}, T\right\}\right) \times \Omega_{ \pm} \\
\rho_{\mu} & \geqq \frac{\delta_{\mu}^{0}}{2} \quad \text { in } \quad\left(0, \min \left\{T_{\mu}^{0}, T\right\}\right) \times \Omega_{ \pm} \\
J^{\psi} & \geqq \frac{1}{2} \kappa^{0} \quad \text { in } \quad\left(0, \min \left\{T_{\mu}^{0}, T\right\}\right) \times \mathbb{R}^{3} .
\end{aligned}
$$

The constant $C_{\mu}^{0}>0$ is bounded above as follows:

$$
C_{\mu}^{0} \leqq C_{\mu}\left(\mathcal{E}_{\mu}^{0}, \frac{1}{\delta^{0}}, \frac{1}{\delta_{\mu}^{0}}, \frac{1}{\kappa^{0}}, E_{g}(1)\right)>0,
$$


where $C_{\mu}(\cdot)$ is a smooth increasing function of its arguments, and may depend on $\mu$.

The time $T_{\mu}^{0}>0$ is bounded below as follows:

$$
T_{\mu}^{0} \geqq T_{\mu}\left(\mathcal{E}_{\mu}^{0}, \frac{1}{\delta^{0}}, \frac{1}{\delta_{\mu}^{0}}, \frac{1}{\kappa^{0}}, E_{g}(1)\right)>0,
$$

where $T_{\mu}(\cdot)$ is a smooth decreasing function of its arguments, and may depend on $\mu$.

Proof. Note that the definition of the linearised equations implies that the pressure and entropy equations (39) and (41) hold, along with the boundary conditions (42)-(43), along with the following additional equations:

$$
\begin{aligned}
& \rho_{\mu}\left(\partial_{t}+u^{\psi} \cdot \nabla\right) \omega+\nabla^{\psi} \rho_{\mu} \times\left(\partial_{t}+u^{\psi} \cdot \nabla\right) u+\rho_{\mu}\left(\varepsilon_{i j k} \partial_{j}^{\psi} u_{l} \partial_{l}^{\psi} u_{k}\right)=\nabla^{\psi} \times g_{1} \\
& \rho_{\mu}\left(\partial_{t}+u^{\psi} \cdot \nabla\right) u+\nabla^{\psi} p=\mu \nabla^{\psi}\left(\rho c^{2} \nabla^{\psi} \cdot u\right)+g_{1}+\mu \nabla^{\psi} \times\left(\rho c^{2} \omega\right) \\
& \quad-\mu \nabla^{\psi} \times\left(\rho c^{2} \nabla^{\psi} \times u\right)
\end{aligned}
$$

in $(0, T) \times \Omega_{ \pm}$and

$$
n \times\left(\nabla^{\psi} \times u_{ \pm}\right)=n \times \omega_{ \pm}
$$

on $(0, T) \times \Gamma$. Thus it remains to show that the velocity equation (40) holds, which will follow immediately from the above if we can show

$$
\omega=\nabla^{\psi} \times u
$$

To do this we take the curl of the Equation (184) and compare it to the equation (183). Using Lemma 16 and the fact that $\psi=\bar{\psi}$ and $u=\bar{u}$, we see that $\nabla^{\psi} \times u$ satisfies the following equation:

$$
\begin{aligned}
\rho_{\mu}\left(\partial_{t}+u^{\psi} \cdot \nabla\right)\left(\nabla^{\psi} \times u\right)= & -\nabla^{\psi} \rho_{\mu} \times\left(\partial_{t}+u^{\psi} \cdot \nabla\right) u-\rho_{\mu}\left(\varepsilon_{i j k} \partial_{j}^{\psi} u_{l} \partial_{l}^{\psi} u_{k}\right) \\
& +\nabla^{\psi} \times g_{1} \\
& +\mu \nabla^{\psi} \times\left(\nabla^{\psi} \times\left(\rho c^{2} \omega\right)\right)-\mu \nabla^{\psi} \\
& \times\left(\nabla^{\psi} \times\left(\rho c^{2} \nabla^{\psi} \times u\right)\right) .
\end{aligned}
$$

Subtracting the curl equation (183) and writing $\xi=\rho c^{2} \nabla^{\psi} \times u-\rho c^{2} \omega$, we have

$$
\rho_{\mu}\left(\partial_{t}+u^{\psi} \cdot \nabla\right)\left(\frac{\xi}{\rho c^{2}}\right)=-\mu \nabla^{\psi} \times\left(\nabla^{\psi} \times \xi\right) .
$$

Multiplying by $\xi$ and integrating by parts separately in $\Omega_{ \pm}$we obtain 


$$
\begin{aligned}
\frac{1}{2} & \frac{\mathrm{d}}{\mathrm{d} t} \int_{\Omega_{ \pm}} \frac{\rho_{\mu}}{\rho c^{2}}|\xi|^{2} \mathrm{~d} x \mp \frac{1}{2} \int_{\Gamma} \frac{\rho_{\mu}}{\rho c^{2}}\left(u^{\psi}\right)_{3}|\xi|^{2} \mathrm{~d} x \\
= & \frac{1}{2} \int_{\Omega_{ \pm}} \partial_{t} \rho_{\mu}|\xi|^{2} \mathrm{~d} x+\frac{1}{2} \int_{\Omega_{ \pm}} \nabla \cdot\left(\rho_{\mu} u^{\psi}\right)|\xi|^{2} \mathrm{~d} x \\
& -\frac{1}{2} \int_{\Omega_{ \pm}} \rho_{\mu} \partial_{t}\left(\frac{1}{\rho c^{2}}\right)|\xi|^{2} \mathrm{~d} x-\frac{1}{2} \int_{\Omega_{ \pm}} \rho_{\mu} u^{\psi} \cdot \nabla\left(\frac{1}{\rho c^{2}}\right)|\xi|^{2} \mathrm{~d} x \\
& \pm \mu \int_{\Gamma} n \times\left(\nabla^{\psi} \times \xi\right) \cdot \xi \mathrm{d} x^{\prime}-\mu \int_{\Omega_{ \pm}}\left|\nabla^{\psi} \times \xi\right|^{2} \mathrm{~d} x \\
& -\mu \int_{\Omega_{ \pm}} \partial_{x_{3}}\left(\frac{\nabla \psi}{J \psi}\right) \times\left(\nabla^{\psi} \times \xi\right) \cdot \xi \mathrm{d} x .
\end{aligned}
$$

Note that using the basic vector identity $(a \times b) \cdot c=(c \times a) \cdot b$ we have $n \times\left(\nabla^{\psi} \times\right.$ $\xi) \cdot \xi=(n \times \xi) \cdot\left(\nabla^{\psi} \times \xi\right)=0$ using the boundary condition (185). Hence, using this together with the fact that $\left.\left(u^{\psi}\right)_{3}\right|_{\Gamma}=0$, we have

$$
\frac{\mathrm{d}}{\mathrm{d} t} \int_{\Omega_{ \pm}}|\xi|^{2} \mathrm{~d} x \leqq \frac{1}{\delta^{0}}\left(C_{U_{ \pm}, f} \int_{\Omega_{ \pm}}|\xi|^{2} \mathrm{~d} x\right)
$$

where the constant $C_{U_{ \pm}, f}<\infty$ depends on $\left\|U_{ \pm}\right\|_{W^{1, \infty}\left((0, T) \times \Omega_{ \pm}\right)}$ and $\|f\|_{W^{2, \infty}((0, T) \times \Gamma)}$. We used Cauchy's inequality to absorb the last term into the second to last term. Note that by definition,

$$
\left.\xi_{ \pm}\right|_{t=0}=0
$$

hence we may apply Gronwall's lemma to conclude that $\xi_{ \pm}=0$. This proves that $\left(U_{+}, U_{-}, f\right)$ is a solution of the $\mu$-approximate equations on the time interval $(0, T)$.

Since $\left.\left(U_{+}, U_{-}, f\right)\right|_{t=0}=\left(U_{+}^{0}, U_{-}^{0}, f^{0}\right)$ and $\left(U_{+}, U_{-}, f\right)$ solves the $\mu-$ approximate equations, it is now clear by definition of the initial time derivatives that

$$
\left.\partial_{t}^{j}\left(U_{+}, U_{-}, f\right)\right|_{t=0}=\partial_{0}^{\mu, g, j}\left(U_{+}^{0}, U_{-}^{0}, f^{0}\right) \text { for } 0 \leqq j \leqq 3 .
$$

Thus we may apply the linearised energy estimate given in Proposition 11 with background state $\left(U_{+}, U_{-}, f\right)$ and rearrange to conclude that, for $t \in(0, T)$,

$$
\mathcal{E}_{L}(t)+\mathcal{E}_{L}(\omega)(t) \leqq M_{\mu}^{0}+K_{\mu}(t)\left(t+\int_{0}^{t} \mathcal{E}_{L}(\tau)+\mathcal{E}_{L}(\omega)(\tau) \mathrm{d} \tau\right)
$$

Now we relate $\mathcal{E}_{L}(t)+\mathcal{E}_{L}(\omega)(t)$ and $\mathcal{E}_{\mu}(t)$ using Lemma 15 to obtain

$$
\mathcal{E}_{\mu}(t) \leqq M_{\mu}^{0}+K_{\mu}(t)\left(t+\int_{0}^{t} \mathcal{E}_{\mu}(\tau) \mathrm{d} \tau\right) \leqq M_{\mu, 0}^{0}+t F_{\mu, 1}\left(M_{\mu, 1}^{0}, \mathcal{E}_{\mu}(t)\right) .
$$

Where $F_{\mu, 1}$ is a smooth increasing function and $M_{\mu, i}^{0}$ has the same form as $M_{\mu}^{0}$. Moreover, we have

$$
\rho \geqq \delta^{0}-t K_{\mu, 2}(t), \quad \rho_{\mu} \geqq \delta_{\mu}^{0}-t K_{\mu, 3}(t), \quad J^{\psi} \geqq \kappa^{0}-t K_{\mu, 4}(t)
$$


on the time interval $(0, T)$ where $K_{\mu, i}$ have the same form as $K_{\mu}$. Note that we can write $K_{\mu, i}(t) \leqq F_{\mu, i}\left(M_{\mu, i}^{0}, \mathcal{E}_{\mu}(t)\right)$ for some smooth increasing functions $F_{\mu, i}$.

We let $T_{\mu}^{0}=\sup \left\{T^{\prime} \in(0, T)\right\}$ such that the following hold for all $t \in\left(0, T^{\prime}\right)$ :

$$
\mathcal{E}_{\mu}(t) \leqq 2 M_{\mu, 0}^{0}, \quad \inf _{\Omega_{ \pm}} \rho \geqq \frac{\delta^{0}}{2}, \quad \inf _{\Omega_{ \pm}} \rho_{\mu} \geqq \frac{\delta_{\mu}^{0}}{2}, \quad \inf _{\mathbb{R}^{3}} J^{\psi} \geqq \frac{1}{2} \kappa^{0},
$$

and the above estimates allow us to immediately derive a lower bound of the required form for $T_{\mu}^{0}$.

Lemma 28. The metric space $Y$ is non-empty for sufficiently large $R^{0}$ where $R^{0}$ depends on the high order energy $\mathcal{E}_{\max }^{0}$ of the initial data $\left(U_{+}^{0}, U_{-}^{0}, f^{0}\right)$, provided we assume $\mathcal{E}_{\max }^{0}<\infty$. More precisely, $R^{0}$ should satisfy

$$
R^{0} \geqq F_{\mu}\left(\mathcal{E}_{\max }^{0}\right),
$$

where $F_{\mu}(\cdot)$ is a given smooth increasing function.

Proof. We define $\left(U_{+}, U_{-}, f\right)$ in terms of the initial data as follows:

$$
p_{ \pm}=\sum_{j=0}^{3} \frac{1}{j !} t^{j} \partial_{0}^{\mu, g, j} p_{ \pm}^{0}, \quad s_{ \pm}=\sum_{j=0}^{3} \frac{1}{j !} t^{j} \partial_{0}^{\mu, g, j} s_{ \pm}^{0}, \quad f=\sum_{j=0}^{3} \frac{1}{j !} t^{j} \partial_{0}^{\mu, g, j} f_{ \pm}^{0} .
$$

Then clearly,

$$
\left.\partial_{t}^{j}\left(p_{ \pm}, s_{ \pm}, f\right)\right|_{t=0}=\partial_{0}^{\mu, g, j}\left(p_{ \pm}^{0}, s_{ \pm}^{0}, f^{0}\right) \text { for } 0 \leqq j \leqq 3 .
$$

Note that having defined $f$ we can now compute $n, \hat{n}$ etc in terms of $f$. Now, following the notation for the rotation matrix $N$ defined in terms of $f$ such that $\left(N^{-1} w\right)_{3}=w \cdot n$ for any vector $w$, as defined in Section 12.3, we set

$$
\stackrel{\curvearrowright}{u}^{0}=\left(N^{0}\right)^{-1} u^{0} \text {. }
$$

Then we define

$$
u(t)=N(t) \sum_{j=0}^{3} \frac{1}{j !} t^{j} \partial_{0}^{\mu, g, j} \stackrel{\curvearrowright}{u}^{0} .
$$

Note that the definition implies that for $0 \leqq j \leqq 3$ we have

$$
\left.\partial_{t}^{j} u\right|_{t=0}=\left.\sum_{k+l=j} \partial_{t}^{k}\left(N(t) N^{-1}(t)\right)\right|_{t=0} \partial_{0}^{\mu, g, l} u^{0}=\partial_{0}^{\mu, g, j} u^{0}
$$

as required. Also,

$$
[u \cdot n]=\left[\left(N^{-1} u\right)_{3}\right]=\sum_{j=0}^{3} \frac{1}{j !} t^{j} \partial_{0}^{\mu, g, j}\left[\hat{u}_{3}^{0}\right]=\sum_{j=0}^{3} \frac{1}{j !} t^{j} \partial_{0}^{\mu, g, j}\left[u^{0} \cdot n^{0}\right]=0,
$$

since the initial data satisfies the compatibility condition (45) up to order 3 .

Since the initial data is smooth, we have $\mathcal{E}_{L}(1)<\infty$, where $\mathcal{E}_{L}(t)$ is the linearised energy associated with the above $\left(U_{+}, U_{-}, f\right)$, so we have $\left(U_{+}, U_{-}, f\right) \in$ $Y$ provided $R^{0} \geqq \mathcal{E}_{L}(1)$, and noting that $\mathcal{E}_{L}(1) \leqq F_{\mu}\left(\mathcal{E}_{\max }^{0}\right)$ for some smooth increasing function $F_{\mu}(\cdot)$ completes the proof. 


\subsubsection{Conclusion of the Proof of the Existence of Solutions to the $\mu$-Approximate Equations}

Proof (The proof of Theorem 3). By Lemma 28, we have that the space $Y$ is non-empty for sufficiently large $R^{0}$ depending on the high-order energy $\mathcal{E}_{\max }^{0}$ of the initial data. Propositions 33 and 34 show that the map $\Phi: Y \rightarrow Y$ defined by $\left(\bar{U}_{+}, \bar{U}_{-}, \bar{f}\right) \mapsto\left(U_{+}, U_{-}, f\right)$ where $\left(U_{+}, U_{-}, f\right)$ is the unique solution of the linearised equations with background state $\left(\bar{U}_{+}, \bar{U}_{-}, \bar{f}\right)$ as given by Proposition 12 is a well-defined contraction, provided that $R^{0}$ and $T$ satisfy the following bounds:

$$
\begin{aligned}
R^{0} & \geqq R_{\mu}\left(\mathcal{E}_{\mu}^{0}, \frac{1}{\delta^{0}}, \frac{1}{\delta_{\mu}^{0}}, \frac{1}{\kappa^{0}}, E_{g}(1)\right) \\
T & \leqq T_{\mu}\left(R^{0}, \mathcal{E}_{\mu}^{0}, \frac{1}{\delta^{0}}, \frac{1}{\delta_{\mu}^{0}}, \frac{1}{\kappa^{0}}, E_{g}(1)\right),
\end{aligned}
$$

where $R_{\mu}$ is a smooth increasing function and $T_{\mu}$ is a smooth decreasing function. Thus we set $R^{0}$ to be the maximum of $R_{\mu}\left(\mathcal{E}_{\mu}^{0}, \frac{1}{\delta^{0}}, \frac{1}{\delta_{\mu}^{0}}, \frac{1}{\kappa^{0}}, E_{g}(1)\right)$ and $F_{\mu}\left(\mathcal{E}_{\max }^{0}\right)$ as given in Lemma 28, then set

$$
T=T_{\mu}\left(R^{0}, \mathcal{E}_{\mu}^{0}, \frac{1}{\delta^{0}}, \frac{1}{\delta_{\mu}^{0}}, \frac{1}{\kappa^{0}}, E_{g}(1)\right) .
$$

We apply Proposition 35 to conclude that $\Phi$ has a fixed point $\left(U_{+}, U_{-}, f\right) \in Y$. We then apply Lemma 27 to conclude that $\left(U_{+}, U_{-}, f\right)$ is a solution of the $\mu$ approximate equations on the time interval $(0, T)$. We also obtain a $T_{\mu}^{0} \in(0, T]$ and $C_{\mu}^{0}>0$ such that

$$
\begin{aligned}
\mathcal{E}_{\mu}\left(\min \left\{T_{\mu}^{0}, T\right\}\right) & \leqq C_{\mu}^{0}<\infty \\
\rho & \geqq \frac{\delta^{0}}{2} \quad \text { in } \quad\left(0, \min \left\{T_{\mu}^{0}, T\right\}\right) \times \Omega_{ \pm} \\
\rho_{\mu} & \geqq \frac{\delta_{\mu}^{0}}{2} \quad \text { in } \quad\left(0, \min \left\{T_{\mu}^{0}, T\right\}\right) \times \Omega_{ \pm} \\
J^{\psi} & \geqq \frac{1}{2} \kappa^{0} \quad \text { in } \quad\left(0, \min \left\{T_{\mu}^{0}, T\right\}\right) \times \mathbb{R}^{3} .
\end{aligned}
$$

The constant $C_{\mu}^{0}>0$ is bounded above as follows:

$$
C_{\mu}^{0} \leqq C_{\mu}\left(\mathcal{E}_{\mu}^{0}, \frac{1}{\delta^{0}}, \frac{1}{\delta_{\mu}^{0}}, \frac{1}{\kappa^{0}}, E_{g}(1)\right)>0,
$$

where $C_{\mu}(\cdot)$ is a smooth increasing function of its arguments, and may depend on $\mu$.

The time $T_{\mu}^{0}>0$ is bounded below as follows:

$$
T_{\mu}^{0} \geqq T_{\mu}\left(\mathcal{E}_{\mu}^{0}, \frac{1}{\delta^{0}}, \frac{1}{\delta_{\mu}^{0}}, \frac{1}{\kappa^{0}}, E_{g}(1)\right)>0,
$$


where $T_{\mu}(\cdot)$ is a smooth decreasing function of its arguments, and may depend on $\mu$.

Redefining $T_{\mu}^{0}=\min \left\{T_{\mu}^{0}, T\right\}$, so that the lower bound becomes

$$
T_{\mu}^{0} \geqq T_{\mu}\left(\mathcal{E}_{\max }^{0}, \frac{1}{\delta^{0}}, \frac{1}{\delta_{\mu}^{0}}, \frac{1}{\kappa^{0}}, E_{g}(1)\right)>0,
$$

where $T_{\mu}(\cdot)$ is a smooth decreasing function of its arguments, we complete the proof.

\subsubsection{Conclusion of the Proof of the Existence of Maximal-Time Solutions to the $\mu$-Approximate Equations}

Proof (The proof of Theorem 4). We need to maximise the time interval of existence of a solution to the $\mu$-approximate equations as given by Theorem 3 . This is almost standard, effectively by taking final data as initial data and applying Theorem 3 again to show that we can extend the time interval of the solution if the energy $\mathcal{E}_{\mu}(t)$ remains bounded. However, there are two minor caveats. The first is that we want to prove that $E_{\mu}(t)$ must blow up as $t$ approaches the maximal time instead of just $\mathcal{E}_{\mu}(t)$. This is straightforward to deduce using the energy estimate given in Proposition 11 together with the relation between $\mathcal{E}_{L}(t)+\mathcal{E}_{L}(\omega)(t)$ and $\mathcal{E}_{\mu}(t)$ given by Lemma 15 , then applying Gronwall's inequality. The second is that is Theorem 3 requires the initial data to be smooth. However, if we examine the proof we see that the only reason for this was to be able to construct a background state for the linearised equations, but once we have a solution on some time interval, we may use it to construct a background state on a larger time interval by Sobolev extension thus overcoming this difficulty. This completes the proof of Theorem 4.

\section{Proof of Uniqueness and Stability}

Here we aim to prove the statement of stability in the main theorem in the fixed domains, Theorem 2 , from which the statement of uniqueness immediately follows. We will use the fact that we have already proved the rest of Theorem 2 . For $i=1,2$, we let $\left(U_{+}^{0, i}, U_{-}^{0, i}, f^{0, i}\right)$ be initial data satisfying the hypotheses of Theorem 2 , and we let $T^{0, i}>0$ be the associated existence times depending on this initial data as given by Theorem 2 . We set $T^{0}=\min \left\{T^{0,1}, T^{0,2}\right\}$. We let $\left(U_{+}^{1}, U_{-}^{1}, f^{1}\right)$ and $\left(U_{+}^{2}, U_{-}^{2}, f^{2}\right)$ be two solutions of the equations in the fixed domains as defined in Definition 11 on the time interval $\left(0, T^{0}\right)$ with initial data $\left(U_{+}^{0, i}, U_{-}^{0, i}, f^{0, i}\right)$ respectively, with the properties

$$
E^{i}\left(T^{0}\right)=: C^{i}<\infty, \quad \inf _{t \in\left(0, T^{0}\right)} \inf _{x \in \Omega_{ \pm}} \rho_{ \pm}^{i}=: \delta^{i}>0, \inf _{t \in\left(0, T^{0}\right)} \inf _{x \in \mathbb{R}^{3}} J^{\psi^{i}}=: \kappa^{i}>0
$$

for $i=1,2$, where the superscript ${ }^{i}$ for $i=1,2$ is used to denote quantities associated with the solutions $\left(U_{+}^{i}, U_{-}^{i}, f^{i}\right)$, and the energy $E(t)$ is defined in Definition 15. We assume that $U^{0,1}-U^{0,2} \in L^{2}\left(\Omega_{ \pm}\right)$and $f^{0,1}-f^{0,2} \in L^{2}(\Gamma)$ so that $E_{\Delta}^{0}<\infty$, where the initial difference energy $E_{\Delta}^{0}$ is defined in Definition 16. 
Notation. In analogy with the proof of the energy estimate proved in Section 9, we will write

$$
K(t)=F\left(E^{1}(t), E^{2}(t), \frac{1}{\delta^{1}}, \frac{1}{\delta^{2}}, \frac{1}{\kappa^{1}}, \frac{1}{\kappa^{2}}\right)
$$

for $t \in\left(0, T^{0}\right]$, where $F$ is a smooth increasing function, and note that

$$
K(t) \leqq K\left(T^{0}\right)=F\left(C^{1}, C^{2}, \frac{1}{\delta^{1}}, \frac{1}{\delta^{2}}, \frac{1}{\kappa^{1}}, \frac{1}{\kappa^{2}}\right)<\infty .
$$

We aim to show that

$$
E_{\Delta}(t) \leqq K(t) E_{\Delta}^{0}+\frac{1}{\varepsilon^{\frac{3}{2}}} K(t) \int_{0}^{t} E_{\Delta}(\tau) \mathrm{d} \tau+\varepsilon^{\frac{1}{2}} K(t) E_{\Delta}(t),
$$

where we are free to choose $\varepsilon \in(0,1]$ and $E_{\Delta}(t)$ is defined in Definition 16 .

The strategy of the proof is just to follow the proof of the $\mu$-independent energy estimate (given in Section 9) with $\mu=0$, where we take the difference of the equations after differentiating (up to 2 times rather than 3 times as in the main energy estimate). We write terms of the form $G\left(U^{1}, \psi^{1}\right) \partial^{\alpha} \partial U^{1}-G\left(U^{2}, \psi^{2}\right) \partial^{\alpha} \partial U^{2}$ as $G\left(U^{1}, \psi^{1}\right) \partial^{\alpha} \partial\left(U^{1}-U^{2}\right)+\left(G\left(U^{1}, \psi^{1}\right)-G\left(U^{2}, \psi^{2}\right)\right) \partial^{\alpha} \partial U^{2}$ and estimate the first term as in the $\mu$-independent energy estimate. The second term is estimated by applying the mean value theorem to $G\left(U^{1}, \psi^{1}\right)-G\left(U^{2}, \psi^{2}\right)$ and using the fact that this is a lower order difference term, and using the higher order energy bound to bound the factor $\partial^{\alpha} \partial U^{2}$. Thus in fact the proof is almost exactly the same as that of the $\mu$-independent energy estimate, hence we will not go through all the details here.

Once we have followed the steps in Section 9 to obtain (186), we proceed as follows. We have

$$
\begin{aligned}
E_{\Delta}(t) & \leqq K(t) E_{\Delta}^{0}+\frac{1}{\varepsilon^{\frac{3}{2}}} K(t) \int_{0}^{t} E_{\Delta}(\tau) \mathrm{d} \tau+\varepsilon^{\frac{1}{2}} K(t) E_{\Delta}(t) \\
& \leqq K\left(T^{0}\right) E_{\Delta}^{0}+\frac{1}{\varepsilon^{\frac{3}{2}}} K\left(T^{0}\right) \int_{0}^{t} E_{\Delta}(\tau) \mathrm{d} \tau+\varepsilon^{\frac{1}{2}} K\left(T^{0}\right) E_{\Delta}(t)
\end{aligned}
$$

where we are free to choose $\varepsilon \in(0,1]$. Choosing $\varepsilon^{\frac{1}{2}}=\frac{1}{2 K\left(T^{0}\right)}$ and rearranging, we obtain

$$
E_{\Delta}(t) \leqq K\left(T^{0}\right) E_{\Delta}^{0}+K\left(T^{0}\right) \int_{0}^{t} E_{\Delta}(\tau) \mathrm{d} \tau,
$$

where we have redefined the function $K(t)$, but it is still of the same form and $K\left(T^{0}\right)<\infty$. Applying Gronwall's lemma we immediately obtain that

$$
E_{\Delta}(t) \leqq K\left(T^{0}\right) E_{\Delta}^{0} \exp \left(K\left(T^{0}\right) t\right)
$$

for $t \in\left(0, T^{0}\right)$. This is the statement of stability. 
Note in particular that this implies that if $\left(U_{+}^{0,1}, U_{-}^{0,1}, f^{0,1}\right)=\left(U_{+}^{0,2}, U_{-}^{0,2}\right.$, $f^{0,2}$ ), then

$$
E_{\Delta}(t)=0
$$

for $t \in\left(0, T^{0}\right)=\left(0, T^{0,1}\right)=\left(0, T^{0,2}\right)$. Thus

$$
\begin{aligned}
\left\|U^{1}-U^{2}\right\|_{L^{2}\left(\Omega_{ \pm}\right)} & =0 \\
\left\|f^{1}-f^{2}\right\|_{L^{2}(\Gamma)} & =0
\end{aligned}
$$

for $t \in\left(0, T^{0}\right)$, and hence $U^{1}=U^{2}$ almost everywhere in $\left(0, T^{0}\right) \times \Omega_{ \pm}$and $f^{1}=$ $f^{2}$ almost everywhere on $\left(0, T^{0}\right) \times \Gamma$. This completes the proof of uniqueness.

\section{Extensions}

\subsection{The Two-Dimensional Case}

Suppose that the flow is independent of one of the horizontal directions, so that we may consider the fluid to occupy some two-dimensional regions $\Omega_{ \pm}(t) \subset \mathbb{R}^{2}$ separated by a one-dimensional surface $\Gamma(t)$. Then in fact exactly the same theorem as Theorem 2 holds, where we replace the three-dimensional space variable with a two-dimensional one by, say, dropping the $x_{1}$ coordinate, then relabelling the coordinates so that $x_{1}$ becomes the horizontal direction and $x_{2}$ the normal direction. Our proof works in exactly the same way.

\subsection{Body Force}

We could easily adapt the proof to include a sufficiently smooth given body force, $h(t, x)$, added to the right hand side of the velocity equation (10). We could even allow the force to be a function of the fluid variables, such as a gravitational force, but with the constraint that it must decay since we are working in the whole space. Terms of zero order can be treated as remainder terms in the main energy estimate and then mollified as we mollified the density to produce the $\mu$-approximate equations. When linearising we may simply replace them with the state about which we linearise.

\subsection{Horizontally Periodic Domain with Top and Bottom Walls}

Instead of working on the unbounded domains $\Omega_{ \pm}^{T}$ with interface $\Gamma^{T}$ as defined in Definition 3, we could work on a horizontally periodic domain with top and bottom walls, by which we mean we redefine

$$
\begin{aligned}
& \Omega_{+}^{T}:=\left\{(t, x) \in(0, T) \times \Omega: x_{3}>f(t, x)\right\} \\
& \Omega_{-}^{T}:=\left\{(t, x) \in(0, T) \times \Omega: x_{3}<f(t, x)\right\} \\
& \Gamma^{T}:=\left\{(t, x) \in(0, T) \times \Omega: x_{3}=f(t, x)\right\}
\end{aligned}
$$


where the fixed domain $\Omega$ is defined as

$$
\Omega:=\left\{(t, x) \in(0, T) \times \mathbb{R}^{3}: x_{3}<D_{+}, x_{3}>-D_{-},-L<x_{1}, x_{2}<L\right\}
$$

for some fixed constants $D_{+}>0, D_{-}>0, L>0$. We impose periodic boundary conditions in the horizontal directions, and we impose the solid wall boundary condition

$$
u_{3}=0
$$

on $(0, T) \times\left\{x_{3}= \pm D_{ \pm}\right\}$. Note that this is exactly the statement that the fluid does not flow through the walls at $x_{3}= \pm D_{ \pm}$since $u_{3}$ is the component of the velocity normal to these walls.

We require that initially the surface $\Gamma^{0}$ does not touch the walls, which means that for a short time by continuity it will still not touch the walls. We note that when constructing the lift $\psi$ of $f$, we want to ensure that $\psi=0$ in a neighbourhood of the walls so that the change of coordinates $\theta$ is the identity in a neighbourhood of the walls. Thus we want to set $\psi=\tilde{\chi}\left(x_{3}\right) f$ where $\tilde{\chi}$ is a smooth cut-off function with sufficiently small support depending on $D_{ \pm}$. But then $\partial_{x_{3}} \psi$ might be quite large, so one way to ensure that $\theta$ remains a diffeomorphism would be to set $\theta(t, x)=\left(t, x^{\prime}, A x_{3}+\psi(t, x)\right)$ for $A$ a sufficiently large positive constant, to ensure that the Jacobian $J^{\psi}:=A+\partial_{x_{3}} \psi$ is uniformly positive. This slight change should not cause any difficulties in adapting the proof of Theorem 1 to the case of a horizontally periodic domain with top and bottom walls. Note that the boundary condition $u_{3}=0$ on $(0, T) \times\left\{x_{3}= \pm D_{ \pm}\right\}$ensures that the boundary terms generated in the energy estimate due to the walls are in fact zero.

Thus we conclude that it would be straightforward to adapt the proof of Theorem 1 to the case of a horizontally periodic domain with top and bottom walls, or indeed a domain which is unbounded horizontally but bounded by walls above and below.

\subsection{Bounded Domain}

The case of a bounded domain was considered by CHENG et al. [6] in the incompressible setting. In this setup, $\mathbb{R}^{3}$ is replaced by a sufficiently smooth simply connected domain $\Omega \subset \mathbb{R}^{3}$ and the interface between the two fluids $\Gamma$ is a closed surface which divides the interior of $\Omega$ into two pieces, $\Omega_{+}$and $\Omega_{-}$, where, say, $\Omega_{+}$is the outer region.

The interior equations and the pressure interface condition remain the same, and the interface $\Gamma$ still moves with the fluid, which in a local chart would still be the condition $\partial_{t} f=u \cdot n$. We assume that the outer boundary of $\Omega$ acts as a fixed wall, so the boundary condition on $\partial \Omega$ is $u \cdot v=0$ where $v$ is normal to $\partial \Omega$. This implies that any boundary terms generated in the energy estimate due to the outer boundary $\partial \Omega$ are lower order terms provided $\Omega$ is a sufficiently smooth domain.

The analysis of this problem requires the introduction of coordinate charts and a partition of unity to define derivatives on $\Gamma$, which adds significant technical complications to the problem. However, since we have not imposed any smallness assumption of the graph of the from $f$ in the above work, it seems likely that there is no fundamental obstruction in adapting the above work to a bounded domain. 


\section{The One-Phase Problem With and Without Surface Tension}

The one-phase problem with and without surface tension has been solved by CoutAND et al. [10], and earlier by Lindblad without surface tension in both the incompressible [18] and compressible [17] cases, so we do not devote a large section to this problem. However, we sketch the details of the results we would obtain for the one-phase problem using the above methods since the exact statements are slightly different to those in these references and the methods used are somewhat different.

\subsection{Statement of the One-Phase Problem}

When we say the one phase problem, we are referring to the problem obtained from the vortex-sheet problem when we replace one of the fluids, say the fluid in the region $\Omega_{+}^{T}$, by vacuum (Fig. 3). The interior equations in $\Omega_{-}^{T}$ remain the same, the interface still moves with the fluid and we replace $p_{+}$by 0 in the pressure interface condition (13). Thus the system of equations can be written as follows, where we drop the subscript - since it is now redundant, so $\Omega^{T}$ refers to $\Omega_{-}^{T}$, etc:

$$
\begin{aligned}
\frac{1}{\rho c^{2}}\left(\partial_{t}+u \cdot \nabla\right) p+\nabla \cdot u & =0 \\
\rho\left(\partial_{t}+u \cdot \nabla\right) u+\nabla p & =0 \\
\left(\partial_{t}+u \cdot \nabla\right) s & =0
\end{aligned}
$$

in $\Omega^{T}$,

$$
\begin{aligned}
\partial_{t} f & =u \cdot n \\
p & =\sigma \nabla^{\prime} \cdot \hat{n}
\end{aligned}
$$

on $\Gamma^{T}$.
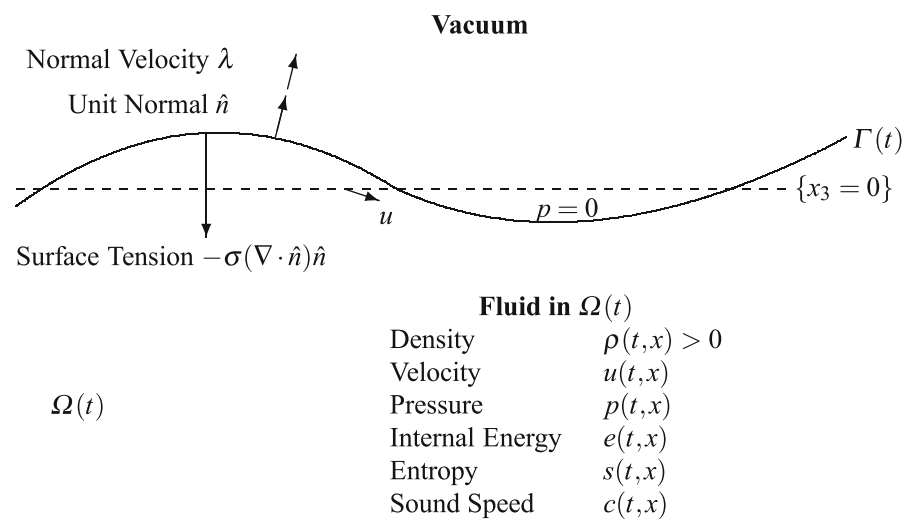

Fig. 3. Perturbation of a flat liquid-vacuum boundary 
If $\sigma>0$ we call this the one-phase problem with surface tension, and if $\sigma=0$ we call it the one-phase problem without surface tension.

The compatibility conditions given in Definition 6 reduce to the following compatibility condition.

Definition 47. We say that initial data $\left(U^{0}, f^{0}\right)$ satisfy the compatibility condition for the system (187)-(191) up to order $k$, for integer $k \geqq 0$, if the following holds for all $x^{\prime} \in \mathbb{R}^{2}$ :

$$
\partial_{0}^{j}\left(\left.p^{0}\right|_{\Gamma^{0}}\right)\left(x^{\prime}\right)=\sigma \partial_{0}^{j}\left(\nabla^{\prime} \cdot \hat{n}^{0}\right)\left(x^{\prime}\right)
$$

for $0 \leqq j \leqq k$, where

$$
\left.p^{0}\right|_{\Gamma^{0}}\left(x^{\prime}\right):=p^{0}\left(x^{\prime}, f^{0}\left(x^{\prime}\right)\right)
$$

Note that in order to relate this to the above work, we will require that the density is bounded below, so it is non-zero at the boundary, and hence physically we would probably be modelling a compressible liquid rather than a gas. One could also replace the vacuum region with a region of constant pressure $\bar{p}>0$, in which case the pressure interface condition would become $p=\sigma \nabla^{\prime} \cdot \hat{n}+\bar{p}$, which would cause no difficulties in the proof since $\bar{p}$ would disappear upon differentiation.

\subsection{The One-Phase Problem With Surface Tension}

Here we assume $\sigma>0$. In this case it is straightforward to adapt the proof of the main theorem, Theorem 1, to prove an analogous result for the one-phase problem. The statement of the result for the one-phase problem with surface tension can be written as follows. Note that the energy is the same as defined in Definition 7 but without the $\Omega_{+}$terms. We remind the reader that this problem was solved by Coutand et al. [10] using Lagrangian coordinates.

Theorem 5. Let $\left(U^{0}, f^{0}\right)$ be initial data with energy $E^{0}<\infty$. Assume that this initial data satisfies the compatibility condition (192) up to order 2. Assume also that the initial density satisfies

$$
\inf _{x \in \Omega^{0}} \rho^{0}(x)=: \delta^{0}>0 .
$$

Then there exists a time $T^{0}>0$ and a solution $(U, f)$ of the system (187)-(191) with $\sigma>0$ on the time interval $\left(0, T^{0}\right)$. Moreover,

$$
E\left(T^{0}\right) \leqq C^{0}, \quad \rho \geqq \frac{\delta^{0}}{2} \quad \text { in } \quad \Omega^{T^{0}} .
$$

The time $T^{0}>0$ is bounded below as follows:

$$
T^{0} \geqq T\left(E^{0}, \frac{1}{\delta^{0}}\right)>0,
$$


where $T(\cdot)$ is a smooth decreasing function which may depend on $\sigma$. Similarly, the constant $C^{0}>0$ is bounded above as follows:

$$
C^{0} \leqq C\left(E^{0}, \frac{1}{\delta^{0}}\right)<\infty,
$$

where $C(\cdot)$ is a smooth increasing function which may depend on $\sigma$.

In addition, the solution $(U, f)$ is unique on the time interval $\left(0, T^{0}\right)$ in the sense that it is the only solution of the equations on the time interval $\left(0, T^{0}\right)$ with initial data $\left(U^{0}, f^{0}\right)$ satisfying the following properties:

$$
E\left(T^{0}\right)<\infty, \quad \inf _{(t, x) \in \Omega^{T^{0}}} \rho>0 .
$$

We avoid repeating all the details of the proof, but we note that there are some significant simplifications particularly in the energy estimate. Indeed in proving the energy estimate, everything is estimated in an exactly analogous way as in Section 9 , except that a large simplification can be made when estimating the highest order time derivative. The boundary term $K_{13}^{\beta}$, which we had to estimate by going back to the equations in the interior, is no longer present at all. In particular, this means that we can use the time derivative $\partial_{t}$ instead of the material time derivative $D_{t}^{u-}$ in this high order time derivative estimate, and we can skip all the estimates dealing with the term $K_{13}^{\beta}$. In fact it becomes possible to estimate the tangential and time derivatives in the same way, provided that we estimate the term corresponding to $I_{2}^{\alpha}$ in the same way as $K_{12}^{\beta}$.

\subsection{The One-Phase Problem Without Surface Tension}

It is also possible under slightly modified assumptions to prove short-time existence and uniqueness for the one-phase problem without surface tension, that is $\sigma=0$. Indeed, all that is required is to prove an energy estimate for the one-phase problem with surface tension that is independent of $\sigma$ (which is the same as proving an energy estimate for the $\mu$-approximate one-phase problem that is independent of $\sigma$ in the case $\mu=0$ ). One could then use weak-* compactness and the Sobolev compact embedding theorem for solutions of the problem with surface tension to pass to the limit $\sigma=0$ and obtain solutions to the problem without surface tension. Note that obtaining a time interval of existence independent of $\sigma$ given a $\sigma$-independent energy estimate would be straightforward assuming that the time interval of existence for the case $\sigma>0$ can be maximised in such a way that the $\sigma$-independent energy blows up $t$ reaches the maximal time.

Note that in the case $\sigma=0$ we no longer obtain an elliptic-type estimate for the front $f$ from the pressure interface condition (191). In fact, to obtain a good estimate for $f$ we need to make an additional assumption on the initial data. That is, the Taylor sign condition should be satisfied as follows:

$$
-\left.\partial_{x_{3}} p^{0}\right|_{\Gamma^{0}} \geqq \lambda^{0}>0
$$


for some $\lambda^{0}>0$. Note this implies by continuity that the Taylor sign condition holds for a short time $T>0$, that is:

$$
-\left.\partial_{x_{3}} p\right|_{\Gamma^{T}} \geqq \frac{\lambda^{0}}{2}>0 .
$$

Note that in the case of an unbounded domain, the requirement $-\left.\partial_{x_{3}} p^{0}\right|_{\Gamma^{0}} \geqq \lambda^{0}>$ 0 conflicts with $\nabla p^{0} \in L^{2}\left(\Omega^{0}\right)$. It may be possible to allow for this by adding a gravitational body force and subtracting a background pressure, but even then it seems that we would need to introduce a bottom wall to prevent the density from tending to infinity. Hence in this section for simplicity we will restrict to the case of a horizontally periodic domain with bottom wall, but we expect the same result to hold for a general sufficiently smooth bounded domain, or a horizontally unbounded domain with bottom wall with gravitational body force after subtracting an appropriate background pressure. We define

$$
\begin{aligned}
& \Omega^{T}:=\left\{(t, x) \in(0, T) \times \Omega: x_{3}<f(t, x)\right\} \\
& \Gamma^{T}:=\left\{(t, x) \in(0, T) \times \Omega: x_{3}=f(t, x)\right\},
\end{aligned}
$$

where the fixed domain $\Omega$ is defined as

$$
\Omega:=\left\{(t, x) \in(0, T) \times \mathbb{R}^{3}: x_{3}>-D,-L<x_{1}, x_{2}<L\right\}
$$

for some fixed constants $D>0, L>0$. We impose periodic boundary conditions in the horizontal directions, and we impose the solid wall boundary condition

$$
u_{3}=0
$$

on $(0, T) \times\left\{x_{3}=-D\right\}$.

The loss of the estimate for the front $f$ from the pressure interface condition (191) means that we should modify our energy so that $U$ and $f$ are estimated to the same order. If we try and do this with an energy with only 3 derivatives, then the remainder terms become impossible to estimate, hence we should increase the order of the derivatives in our energy by one. If we include the terms in our energy that we would have in the case $\sigma>0$, but this time multiplied by the appropriate factor of $\sigma$ so that we can obtain a $\sigma$-independent estimate for this energy, we obtain the following definition.

Definition 48. Define the $\sigma$-independent energy $E_{\sigma}:(0, T] \rightarrow[0, \infty]$ by

$$
\begin{aligned}
E_{\sigma}(t)= & \underset{\tau \in(0, t)}{\operatorname{ess} \sup \|U\|_{L^{\infty}(\Omega(\tau))}^{2}}+\sum_{1 \leqq|\alpha| \leqq 4} \operatorname{ess} \sup \left\|\partial^{\alpha} U\right\|_{L^{2}(\Omega(\tau))}^{2}+\underset{\tau \in(0, t)}{\operatorname{ess} \sup \|f\|_{L^{\infty}\left(\mathbb{R}^{2}\right)}^{2}} \\
& +\sum_{1 \leqq|\alpha| \leqq 4} \operatorname{ess} \sup \left\|\partial^{\alpha} f\right\|_{L^{2}\left(\mathbb{R}^{2}\right)}^{2}+\sigma \sum_{1 \leqq|\alpha| \leqq 4} \operatorname{ess} \sup \left\|\partial^{\alpha} f\right\|_{H^{1}\left(\mathbb{R}^{2}\right)}^{2} \\
& +\sigma^{2} \sum_{j=0}^{3} \operatorname{ess~sup}_{\tau \in(0, t)}\left\|\nabla^{\prime} \partial_{t}^{j} f\right\|_{H^{4.5-j}\left(\mathbb{R}^{2}\right)}^{2} .
\end{aligned}
$$

We allow the case $\sigma=0$, in which case the energy is written as $E_{0}(t)$.

The initial energy $E_{\sigma}^{0}$ of the initial data is defined in the obvious way, replacing time derivatives in the above energy by initial time derivatives. 
The statement of the result for the one-phase problem without surface tension in a horizontally periodic domain with bottom wall can be written as follows. We remind the reader that this problem was originally solved by LINDBLAD [17].

Theorem 6. Let $\left(U^{0}, f^{0}\right)$ be initial data with energy $E_{0}^{0}<\infty$. Assume that this initial data satisfies the compatibility condition (192) up to order 3 with $\sigma=0$, plus the compatibility condition associated with the bottom wall,

$$
\left.\partial_{0}^{j} u_{3}^{0}\right|_{x_{3}=-D}=0 \text { for } 0 \leqq j \leqq 3 .
$$

Assume also that the initial density satisfies

$$
\inf _{x \in \Omega^{0}} \rho^{0}(x)=: \delta^{0}>0
$$

and that the Taylor sign condition

$$
-\left.\inf _{x \in \Gamma^{0}} \partial_{x_{3}} p^{0}\right|_{\Gamma^{0}}=: \lambda^{0}>0
$$

is satisfied, and that the initial surface and bottom wall satisfy:

$$
\operatorname{dist}\left(\Gamma^{0},\left\{x_{3}=-D\right\}\right)=: \eta^{0}>0 .
$$

Then there exists a time $T^{0}>0$ and a solution $(U, f)$ of the system (187)-(191) with $\sigma=0$ plus (194) on the time interval $\left(0, T^{0}\right)$. Moreover,

$$
\begin{aligned}
E_{0}\left(T^{0}\right) \leqq C^{0}, \quad \rho \geqq \frac{\delta^{0}}{2} \text { in } \Omega^{T^{0}}, \\
-\partial_{x_{3}} p \geqq \frac{\lambda^{0}}{2} \text { on } \quad \Gamma^{T^{0}}, \quad \inf _{t \in\left(0, T^{0}\right)} \operatorname{dist}\left(\Gamma(t),\left\{x_{3}=-D\right\}\right) \geqq \frac{\eta^{0}}{2} .
\end{aligned}
$$

The time $T^{0}>0$ is bounded below as follows:

$$
T^{0} \geqq T\left(E_{0}^{0}, \frac{1}{\delta^{0}}, \frac{1}{\lambda^{0}}, \frac{1}{\eta^{0}}\right)>0 .
$$

where $T(\cdot)$ is a smooth decreasing function. Similarly, the constant $C^{0}>0$ is bounded above as follows:

$$
C^{0} \leqq C\left(E_{0}^{0}, \frac{1}{\delta^{0}}, \frac{1}{\lambda^{0}}, \frac{1}{\eta^{0}}\right)<\infty,
$$

where $C(\cdot)$ is a smooth increasing function.

In addition, the solution $(U, f)$ is unique on the time interval $\left(0, T^{0}\right)$ in the sense that it is the only solution of the equations on the time interval $\left(0, T^{0}\right)$ with initial data $\left(U^{0}, f^{0}\right)$ satisfying the following properties:

$$
\begin{aligned}
& E\left(T^{0}\right)<\infty, \quad \inf _{(t, x) \in \Omega^{T^{0}}} \rho>0, \\
& -\quad \inf _{(t, x) \in \Gamma^{T^{0}}} \partial_{x_{3}} p>0, \quad \inf _{t \in\left(0, T^{0}\right)} \operatorname{dist}\left(\Gamma(t),\left\{x_{3}=-D\right\}\right)>0 .
\end{aligned}
$$


We sketch some details of how to obtain the $\sigma$-independent energy estimate. Firstly, we should use the corrected unknowns as defined in (72) throughout the energy estimate in order to remove the highest order terms in $\psi$ from the differentiated equations, as we did for the time derivative estimate in Section 9.10. In fact the time derivatives are now estimated in the same way as the tangential derivatives, provided we estimate the term corresponding to $I_{2}^{\alpha}$ in the same way as $K_{12}^{\beta}$. The boundary term that we obtain during the time and tangential estimates using the corrected unknowns is

$$
-K^{\alpha}=\int_{0}^{t} \int_{\Gamma} n \cdot u^{\alpha} p^{\alpha} \mathrm{d} x^{\prime} \mathrm{d} \tau=: K_{1}^{\alpha}-K_{2}^{\alpha}-K_{3}^{\alpha}+K_{4}^{\alpha}
$$

where

$$
\begin{aligned}
K_{1}^{\alpha} & =\int_{0}^{t} \int_{\Gamma} n \cdot \partial^{\alpha} u \partial^{\alpha} p \mathrm{~d} x^{\prime} \mathrm{d} \tau \\
K_{2}^{\alpha} & =\int_{0}^{t} \int_{\Gamma} n \cdot \partial^{\alpha} u \partial^{\alpha} f \partial_{3} p \mathrm{~d} x^{\prime} \mathrm{d} \tau \\
K_{3}^{\alpha} & =\int_{0}^{t} \int_{\Gamma} n \cdot \partial^{\alpha} p \partial^{\alpha} f \partial_{3} u \mathrm{~d} x^{\prime} \mathrm{d} \tau \\
K_{4}^{\alpha} & =\int_{0}^{t} \int_{\Gamma} n \cdot \partial^{\alpha} f \partial_{3} p \partial^{\alpha} f \partial_{3} u \mathrm{~d} x^{\prime} \mathrm{d} \tau .
\end{aligned}
$$

Note that $K_{4}^{\alpha}$ can be estimated directly by $t K(t)$ using notation analogous to that in Section 9 . The term $K_{3}^{\alpha}$ can also be estimated by $t K(t)$ after using (191) to replace $\partial^{\alpha} p$ by $\sigma \partial^{\alpha} \nabla^{\prime} \cdot \hat{n}$ and integrating by parts in space. The term $K_{1}^{\alpha}$ can be estimated as before, again by using (191) to replace $\partial^{\alpha} p$ by $\sigma \partial^{\alpha} \nabla^{\prime} \cdot \hat{n}$.

Now let us consider $K_{2}^{\alpha}$. Using the equation for the front (190), we obtain

$$
\begin{aligned}
K_{2}^{\alpha} & =\int_{0}^{t} \int_{\Gamma} \partial^{\alpha}(u \cdot n) \partial^{\alpha} f \partial_{3} p \mathrm{~d} x^{\prime} \mathrm{d} \tau-\int_{0}^{t} \int_{\Gamma} u \cdot \partial^{\alpha} n \partial^{\alpha} f \partial_{3} p \mathrm{~d} x^{\prime} \mathrm{d} \tau+R \\
& =\int_{0}^{t} \int_{\Gamma} \partial^{\alpha} \partial_{t} f \partial^{\alpha} f \partial_{3} p \mathrm{~d} x^{\prime} \mathrm{d} \tau+\int_{0}^{t} \int_{\Gamma} u \cdot \nabla^{\prime} \partial^{\alpha} f \partial^{\alpha} f \partial_{3} p \mathrm{~d} x^{\prime} \mathrm{d} \tau+R
\end{aligned}
$$

where we are using $R$ to denote a generic remainder term satisfying $|R| \leqq t K(t)$ and we are not being very precise with the remainder terms. We use the product rule and apply the divergence theorem to the second term to obtain

$$
\begin{aligned}
-K_{2}^{\alpha} & =-\left.\frac{1}{2} \int_{\Gamma}\left|\partial^{\alpha} f\right|^{2} \partial_{3} p \mathrm{~d} x^{\prime}\right|_{0} ^{t}-\frac{1}{2} \int_{0}^{t} \int_{\Gamma} \nabla^{\prime} \cdot\left(u\left|\partial^{\alpha} f\right|^{2} \partial_{3} p\right) \mathrm{d} x^{\prime} \mathrm{d} \tau+R \\
& \geqq-\frac{1}{2} \int_{\Gamma}\left|\partial^{\alpha} f\right|^{2} \partial_{3} p \mathrm{~d} x^{\prime}-M^{0}-t K(t) .
\end{aligned}
$$

Thus the Taylor sign condition $-\left.\partial_{3} p\right|_{\Gamma} \geqq \frac{\lambda^{0}}{2}$ ensures we have an estimate for $\left\|\partial^{\alpha} f\right\|_{L^{2}(\Gamma)}^{2}$.

The rest of the energy estimate is much the same as in Section 9, except we use the corrected unknowns throughout. The estimate of the unknowns given an 
estimate for the corrected unknowns is simple given that we have 4 derivatives in our energy, and is obtained as follows using the Sobolev embedding theorem:

$$
\begin{aligned}
\left\|\partial^{\alpha} U\right\|_{L^{2}(\Omega)}^{2} & \leqq C\left\|U^{\alpha}\right\|_{L^{2}(\Omega)}^{2}+\left\|\partial^{\alpha} \psi\right\|_{L^{2}(\Omega)}^{2}\left\|\frac{\partial_{x_{3}} U}{J^{\psi}}\right\|_{L^{\infty}(\Omega)}^{2} \\
& \leqq C\left\|U^{\alpha}\right\|_{L^{2}(\Omega)}^{2}+C\left\|\partial^{\alpha} f\right\|_{L^{2}(\Gamma)}^{2}\left(M^{0}+\int_{0}^{t}\left\|\partial_{t}\left(\frac{\partial_{x_{3}} U}{J^{\psi}}\right)\right\|_{H^{2}(\Omega)}^{2} \mathrm{~d} \tau\right) \\
& \leqq C\left\|U^{\alpha}\right\|_{L^{2}(\Omega)}^{2}+\left\|\partial^{\alpha} f\right\|_{L^{2}(\Gamma)}^{2}\left(M^{0}+t K(t)\right) .
\end{aligned}
$$

This completes our sketch of the proof of Theorem 6 .

\section{Appendix}

\subsection{A Hodge Decomposition Based Estimate}

The following estimate based on Hodge decomposition allows us to separate an estimate for first order derivatives of a vector field into an estimate of the curl, divergence, and normal component on the boundary.

Note that in this section, $f \in C\left([0, T] \times W^{2, \infty}\left(\mathbb{R}^{2}\right)\right), \psi=L^{a} f$ for some $a \geqq 0$ as defined in Definition 9 , the domains $\Omega_{ \pm}$and $\Gamma$ are defined in Definition $8, n=\left(-\nabla^{\prime} f, 1\right), J^{\psi}$ and the notation $\nabla^{\psi}$ are defined in Definition 10, and $J^{\psi} \geqq \kappa>0$ for some constant $\kappa>0$.

First we write the Hodge decomposition in our setting.

Lemma 29. Fix $t \in[0, T]$. Let $w \in C^{2}\left(\Omega_{ \pm} ; \mathbb{R}^{3}\right)$. Then

$$
\Delta^{\psi} w=\nabla^{\psi}\left(\nabla^{\psi} \cdot w\right)-\nabla^{\psi} \times\left(\nabla^{\psi} \times w\right)
$$

where

$$
\left(\Delta^{\psi} w\right)_{i}:=\nabla^{\psi} \cdot\left(\nabla^{\psi} w_{i}\right) .
$$

Proof. This is a simple consequence of the formula (35), which states

$$
\nabla a=\left(\nabla^{\psi}(a \circ \theta)\right) \circ \theta^{-1}
$$

where the diffeomorphisms $\theta_{ \pm}:(0, T) \times \Omega_{ \pm} \rightarrow \Omega_{ \pm}^{T}$ are defined in terms of $f$ in Lemma 1 , plus the well-known vector identity

$$
\Delta z=\nabla(\nabla \cdot z)-\nabla \times(\nabla \times z) .
$$

Proposition 36. Fix $t \in[0, T]$. Let $w_{ \pm} \in L^{2}\left(\Omega_{ \pm} ; \mathbb{R}^{3}\right)$ with $\nabla^{\psi} \cdot w \in L^{2}\left(\Omega_{ \pm}\right)$, $\nabla^{\psi} \times w \in L^{2}\left(\Omega_{ \pm} ; \mathbb{R}^{3}\right)$ and $w_{ \pm} \cdot n \in H^{0.5}(\Gamma)$. Then $w_{ \pm} \in H^{1}\left(\Omega_{ \pm}\right)$with

$$
\begin{aligned}
\|w\|_{H^{1}\left(\Omega_{ \pm}\right)} \leqq & C\left(1+\left\|\nabla^{\prime} f\right\|_{W^{1, \infty}(\Gamma)}^{3}\right) \\
& \times\left(\|w\|_{L^{2}\left(\Omega_{ \pm}\right)}+\left\|\nabla^{\psi} \cdot w\right\|_{L^{2}\left(\Omega_{ \pm}\right)}+\left\|\nabla^{\psi} \times w\right\|_{L^{2}\left(\Omega_{ \pm}\right)}\right. \\
& \left.+\left\|w_{ \pm} \cdot n\right\|_{H^{0.5}(\Gamma)}\right) .
\end{aligned}
$$


Proof. Note we may assume $w \in C_{c}^{\infty}\left(\Omega_{ \pm}\right)$else we may approximate $w$ by a sequence of $C_{c}^{\infty}\left(\Omega_{ \pm}\right)$functions which converges to $w$ pointwise, and for which the norms on the right hand side are bounded by those of $w$ up to a constant, from which we obtain that $w \in H^{1}\left(\Omega_{ \pm}\right)$with the above estimate.

We dot the formula (199) with $w_{ \pm}$, integrate over $\Omega_{ \pm}$and use integration by parts. This gives us a term involving $\sum_{j=1}^{3} \int_{\Omega_{ \pm}}\left|\partial_{j}^{\psi} w\right|^{2}$, a term involving $\int_{\Omega_{ \pm}}\left|\nabla^{\psi} \times w\right|^{2} \mathrm{~d} x$, a term involving $\int_{\Omega_{ \pm}}\left|\nabla^{\psi} \cdot w\right|^{2} \mathrm{~d} x$ and some boundary terms. Using some vector identities, $H^{0.5}-H^{-0.5}$ estimates and the Sobolev trace lemma allows us to estimate the boundary term as required.

\subsection{Products and Compositions in Sobolev Spaces}

The following lemma is very useful for proving chain and product rules in Sobolev spaces. This is important for obtaining high-order energy estimates.

Lemma 30. Let $p \in[1, \infty], \Omega \subset \mathbb{R}^{d}$ with $d \geqq 1$ be a domain where the standard Sobolev embedding holds and let $m>\frac{d}{p}$ be an integer. Let $0 \leqq m_{i} \leqq m$ be integers for $1 \leqq i \leqq n$ with $\sum_{i=1}^{n} m_{i} \geqq(n-1) m$ and let $u_{i} \in W^{m_{i}, p}(\Omega)$. Then $\prod_{i=1}^{n} u_{i} \in L^{p}(\Omega)$ and

$$
\left\|\prod_{i=1}^{n} u_{i}\right\|_{L^{p}(\Omega)} \leqq C \prod_{i=1}^{n}\left\|u_{i}\right\|_{W^{m_{i}, p}(\Omega)}
$$

Proof. The proof is straightforward using Holder's inequality and the Sobolev embedding theorem.

Lemma 31. Let $s \in[0,1]$ and let $r>\frac{d}{2}$ where $d \geqq 1$ is an integer. Let $a \in$ $H^{s+r}\left(\mathbb{R}^{d}\right)$ and $u \in H^{s}\left(\mathbb{R}^{d}\right)$. Then au $\in H^{s}\left(\mathbb{R}^{d}\right)$ and

$$
\langle D\rangle^{s}(a u)=a\langle D\rangle^{s} u+R(a, u)
$$

where the remainder $R(a, u)$ satisfies

$$
\|R(a, u)\|_{L^{2}\left(\mathbb{R}^{d}\right)} \leqq C\|a\|_{H^{s+r}\left(\mathbb{R}^{d}\right)}\|u\|_{L^{2}\left(\mathbb{R}^{d}\right)}
$$

for some $C>0$.

Proof. First of all, assume $a, u \in C_{c}^{\infty}\left(\mathbb{R}^{d}\right)$. We define $R(a, u)$ by

$$
\langle D\rangle^{s}(a u)-a\langle D\rangle^{s} u=R(a, u) .
$$

Taking Fourier transforms, and using the fact that products become convolutions, we have

$$
\begin{aligned}
\langle\xi\rangle^{s}(\hat{a} * \hat{u})(\xi)-\left(\hat{a} *\left(\langle\eta\rangle^{s} \hat{u}\right)\right)(\xi) & =\hat{R}(\xi) \\
\int_{\mathbb{R}^{d}} \hat{a}(\xi-\eta) \hat{u}(\eta)\left(\langle\xi\rangle^{s}-\langle\eta\rangle^{s}\right) \mathrm{d} \eta & =\hat{R}(\xi) .
\end{aligned}
$$


Hence,

$$
|\hat{R}(\xi)| \leqq \int_{\mathbb{R}^{d}}|\hat{a}(\xi-\eta)||\hat{u}(\eta)|\left|\langle\xi\rangle^{s}-\langle\eta\rangle^{s}\right| \mathrm{d} \eta .
$$

Now we use the inequality

$$
\left|\langle\xi\rangle^{s}-\langle\eta\rangle^{s}\right| \leqq C\langle\xi-\eta\rangle^{s},
$$

which holds for some $C>0$ provided that $s \in[0,1]$. We obtain

$$
\begin{aligned}
|\hat{R}(\xi)| & \leqq C \int_{\mathbb{R}^{d}}|\hat{a}(\xi-\eta)||\hat{u}(\eta)|\langle\xi-\eta\rangle^{s} \mathrm{~d} \eta \\
& =C\left(\langle\eta\rangle^{s}|\hat{a}|\right) *|\hat{u}| .
\end{aligned}
$$

Applying Young's inequality for convolutions, we have

$$
\begin{aligned}
\|\hat{R}(\xi)\|_{L^{2}\left(\mathbb{R}^{d}\right)} & \leqq C\left\|\langle\xi\rangle^{s} \hat{a}\right\|_{L^{1}\left(\mathbb{R}^{d}\right)}\|\hat{u}\|_{L^{2}\left(\mathbb{R}^{d}\right)} \\
& =C\left\|\langle\xi\rangle^{s+r} \hat{a}\langle\xi\rangle^{-r}\right\|_{L^{1}\left(\mathbb{R}^{d}\right)}\|u\|_{L^{2}\left(\mathbb{R}^{d}\right)} \\
& \leqq C\left\|\langle\xi\rangle^{s+r} \hat{a}\right\|_{L^{2}\left(\mathbb{R}^{d}\right)}\left\|\langle\xi\rangle^{-r}\right\|_{L^{2}\left(\mathbb{R}^{d}\right)}\|u\|_{L^{2}\left(\mathbb{R}^{d}\right)} \\
& \leqq C\|a\|_{H^{s+r}\left(\mathbb{R}^{d}\right)}\|u\|_{L^{2}\left(\mathbb{R}^{d}\right)},
\end{aligned}
$$

where we have used Holder's inequality and the fact that $\langle\xi\rangle^{-r} \in L^{2}\left(\mathbb{R}^{d}\right)$ for $r>\frac{d}{2}$. Hence,

$$
\|R(a, u)\|_{L^{2}\left(\mathbb{R}^{d}\right)} \leqq C\|a\|_{H^{s+r}\left(\mathbb{R}^{d}\right)}\|u\|_{L^{2}\left(\mathbb{R}^{d}\right)},
$$

as claimed. If $a$ and $u$ are not smooth then we use approximation by mollification.

Acknowledgments. I would like to thank my supervisor, Professor Gui-Qiang Chen, for guiding me towards the problem considered here. I would also like to thank Steve Shkoller for some helpful conversations on this topic. Finally, I would like to thank the EPSRC for funding my research.

Open Access This article is distributed under the terms of the Creative Commons Attribution 4.0 International License (http://creativecommons.org/licenses/by/4.0/), which permits unrestricted use, distribution, and reproduction in any medium, provided you give appropriate credit to the original author(s) and the source, provide a link to the Creative Commons license, and indicate if changes were made.

\section{References}

1. Adams, R.A., Fournier, J.J.F.: Sobolev spaces. Pure and Applied Mathematics (Amsterdam), vol. 140, 2nd edn. Elsevier/Academic Press, Amsterdam, 2003

2. Alinhac, S.: Existence d'ondes de raréfaction pour des systèmes quasi-linéaires hyperboliques multidimensionnels. Commun. Partial Differ. Equ. 14(2), 173-230 (1989). doi:10.1080/03605308908820595

3. Benzoni-Gavage, S., Serre, D.: Multidimensional hyperbolic partial differential equations. First-order systems and applications. In: Oxford Mathematical Monographs. The Clarendon Press Oxford University Press, Oxford (2007)

4. Chen, G.Q., WANG, Y.G.: Existence and stability of compressible current-vortex sheets in three-dimensional magnetohydrodynamics. Arch. Rat. Mech. Anal. 187(3), 369-408 (2008). doi:10.1007/s00205-007-0070-8 
5. Chen, G.Q., WANG, Y.G.: Characteristic discontinuities and free boundary problems for hyperbolic conservation laws. Nonlinear Partial Differential Equations-The Abel Symposium 2010, vol. 7. Abel Symposia, Vol. 7 (Eds. Holden H. and Karlsen K.). Springer, Berlin, 2012

6. Cheng, C.H.A., Coutand, D., Shkoller, S.: On the motion of vortex sheets with surface tension in three-dimensional Euler equations with vorticity. Commun. Pure Appl. Math. 61(12), 1715-1752 (2008). doi:10.1002/cpa.20240

7. Coulombel, J.F., Morando, A., Secchi, P., Trebeschi, P.: A priori estimates for 3D incompressible current-vortex sheets. Commun. Math. Phys. 311(1), 247-275 (2012). doi:10.1007/s00220-011-1340-8

8. Coulombel, J.F., Secchi, P.: The stability of compressible vortex sheets in two space dimensions. Indiana Univ. Math. J. 53(4), 941-1012 (2004). doi:10.1512/iumj.2004. 53.2526

9. Coulombel, J.F., Secchi, P.: Nonlinear compressible vortex sheets in two space dimensions. Ann. Sci. Éc. Norm. Supér. (4) 41(1), 85-139 (2008)

10. Coutand, D., Hole, J., ShKoller, S.: Well-posedness of the free-boundary compressible 3-d euler equations with surface tension and the zero surface tension limit. SIAM J. Math. Anal. 45(6), 3690-3767 (2013). doi:10.1137/120888697

11. Coutand, D., Shroller, S.: Well-posedness of the free-surface incompressible Euler equations with or without surface tension. J. Am. Math. Soc. 20(3), 829-930 (2007). doi:10.1090/S0894-0347-07-00556-5

12. Coutand, D., Shkoller, S.: A simple proof of well-posedness for the free-surface incompressible Euler equations. Discrete Contin. Dyn. Syst. Ser. S 3(3), 429-449 (2010). doi:10.3934/dcdss.2010.3.429

13. Coutand, D., Shroller, S.: Well-posedness in smooth function spaces for the movingboundary three-dimensional compressible Euler equations in physical vacuum. Arch. Rat. Mech. Anal. 206(2), 515-616 (2012). doi:10.1007/s00205-012-0536-1

14. Evans, L.C.: Partial differential equations. Graduate Studies in Mathematics, vol. 19, 2nd edn. American Mathematical Society, Providence, 2010

15. Fejer, J.A., Miles, J.W.: On the stability of a plane vortex sheet with respect to threedimensional disturbances. J. Fluid Mech. 15, 335-336 (1963)

16. JANG, J., MAsmoudi, N.: Well-posedness of compressible euler equations in a physical vacuum. Commun. Pure Appl. Math. 68(1), 61-111 (2015)

17. LindBLAD, H.: Well posedness for the motion of a compressible liquid with free surface boundary. Commun. Math. Phys. 260(2), 319-392 (2005)

18. Lindblad, H.: Well-posedness for the motion of an incompressible liquid with free surface boundary. Ann. Math. 162(1), 109-194 (2005)

19. Miles, J.W.: On the disturbed motion of a plane vortex sheet. J. Fluid Mech. 4, 538-552 (1958)

20. Trakhinin, Y.: The existence of current-vortex sheets in ideal compressible magnetohydrodynamics. Arch. Rat. Mech. Anal. 191(2), 245-310 (2009). doi:10.1007/s00205008-0124-6

\author{
Merton College, \\ Oxford OX1 4JD, \\ UK. \\ e-mail: ben.stevens@merton.oxon.org
}

(Received August 30, 2014 / Accepted May 6, 2016)

Published online June 16, 2016 - (C) The Author(s) (2016)

This article is published with open access at Springerlink.com 